\title{
P1 Work up to rule out perioperative myocardial infarction: is it overused?
}

\section{SK Appavu, TR Haley, A Khorasani and SR Patel}

Departments of Surgery and Anesthesiology, Cook County Hospital and the Department of Surgery, University of Illinois College of Medicine, Chicago, Illinois, USA

The awareness of the diagnostic difficulty and the documented high mortality risk of perioperative myocardial infarction (PMI) has led to the wide use of work up to rule out PMI after major noncardiac operations. This has caused stable postoperative patients to be kept in monitored hospital beds for extended periods of time and to be subjected to additional tests. We hypothesized that the mortality of PMI is high and, therefore, the wide use of postoperative work up to identify these patients is justifiable. We performed the following study to prove our hypothesis. All patients in the recovery room after major noncardiac operations who underwent work up to rule out PMI were identified and followed. The PMI work up included care in an electronically monitored unit, physical assessment, continuous ECG monitoring, and three 12lead electrocardiograms and cardiac enzymes obtained at six to eight hour intervals. Data collection included patient demographics; preoperative cardiac risk factors; incidence of intraoperative hypotension, hemorrhage and ECG changes; type of anesthesia and operative procedures and their durations; postoperative ECG and cardiac enzyme results; the incidence of PMI and patient outcome.
Two hundred patients were studied; 85 males and 115 females. Their mean age was 62.9 years. Preexisting conditions included hypertension in 162 patients, peripheral arterial disease in 102, diabetes mellitus in 97, angina in 30 , previous myocardial infarction in 41 , and smoking in 107. Of 200 patients, 164 had an abnormal preoperative ECG. Vascular operations were performed in 104 patients, nonvascular abdominal operations in 48 , and other operations in the remaining 48. Intraoperatively, hypotension occurred in 29 patients, blood loss of $>500 \mathrm{ml}$ in 25 and ECG changes in 10 . There were no deaths. PMl occurred in 5/200 (2.5\%) patients. Four had undergone vascular operations and one had had an abdominal operation. The mean age of the patients with PMI was 64.2 years. The duration of operation and blood loss were similar to those of patients without PMI. None of these patients developed cardiac failure or cardiogenic shock and none of them died.

Conclusion: The incidence of PMl among patients undergoing noncardiac surgery is low and its mortality is negligible. Physicians should become more selective in the use of monitored beds and in the ordering of a work up to rule out PMI.

P2 Relationship between cardiac troponin I (cTnl) release during cardiac operations and myocardial cell death

JF Vazquez-Jimenez*, Ma Qing ${ }^{\dagger}$, B Klosterhalfen $¥$, O Liakopoulos*, G von Bernuth†, BJ Messmer* and M-C Seghaye ${ }^{\dagger}$

Department. of Cardiac Surgery ${ }^{*}$, Pediatric Cardiology ${ }^{\dagger}$, Pathology ${ }^{\ddagger}$, Aachen University of Technology, Pauwelsstrasse. 30, D-52057, Aachen, Germany

Aims: To study the relationship between myocardial release of cTnl and myocardial cell death as assessed by the amount of apoptosis and necrosis after cardiac surgery.

Methods: Eighteen young pigs were operated on with standardized cardiopulmonary bypass (CPB). Release of cTnl in the cardiac lymph (CL), coronary sinus (CS), and arterial blood $(A)$ was related to postoperative myocardial cell death by both necrosis and apoptosis. Apoptotic cells were detected by a TUNEL detection kit. Necrotic cells were counted by light microscopy.

Results: In all animals, cTnl was significantly released and reached peak values observed simultaneously in A (cTnl,
20.1 $\pm 2.6 \mathrm{ng} / \mathrm{ml}$ ) (mean \pm SEM), CS $(19.5 \pm 3.2 \mathrm{ng} / \mathrm{ml}$ ) and $\mathrm{CL}(5202 \pm 2500 \mathrm{ng} / \mathrm{ml})$. Percentage of total myocardial cell death was $3.1 \pm 0.5 \%$, including $1.2 \pm 0.35 \%$ necrosis and $1.9 \pm 0.5 \%$ apoptosis. cTnl release during and after CPB did not correlate with the degree of myocardial apoptosis or necrosis.

Conclusion: Cardiac operations with CPB are related to myocardial cell damage including myocardial cell death due to both necrosis and apoptosis. As the loss of cTnl is not related to the amount of cell death, our results suggest that increased cardiac myocyte membrane permeability more than cell death is responsible for intraoperative and postoperative $\mathrm{cTn}$ l release. 


\section{P3 Evaluation of thoracic fluid contents in patients with acute myocardial infarction}

\section{H Okawa, A Suzuki, I Sakai, H Tsubo, H Ishihara and A Matsuki}

University of Hirosaki School of Medicine, Department of Anesthesiology, 5 Zaifucho, Hirosaki, 036-8562 Japan

It is common to evaluate patients with acute myocardial infarction (AMI) according to the Forrester classification. A high PCWP value is a good predictor of pulmonary congestion although there is no documented correlation between PCWP and the degree of pulmonary congestion in patients with normal PCWP. In this study, we sought to investigate the correlation between (1) PCWP and thoracic fluid contents (TFC) as an indicator of pulmonary congestion, (2) PCWP and cardiac index $(\mathrm{Cl})$ and (3) $\mathrm{Cl}$ and TFC in patients with AMI with PCWP values $<18 \mathrm{mmHg}$. The thoracic electrical bioimpedance (TEB) method was used to measure TFC and $\mathrm{Cl}$. $\mathrm{Cl}$ was also measured by the thermodilution (TD) method.

Thirty-six patients with a diagnosis of AMI were included. Patients were monitored with the TEB device (BioZ System, CardioDynamics International Co, USA) and the pulmonary artery (PA) catheter, in addition to conventional monitoring. We measured $\mathrm{Cl}$ by means of TEB and thermodilution (TD) methods (Cl-TEB and Cl-TD, respectively) and compared values obtained from each patient with those two methods. We also measured PCWP with the PA catheter, and TFC values as an indicator of pulmonary congestion with the TEB device. The correlation between
PCWP and $\mathrm{Cl}, \mathrm{PCWP}$ and TFC, $\mathrm{Cl}$ and TFC were also examined.

Patients were 29 males and 7 females and age was $65 \pm 11$ years (mean $\pm S D$ ). All the patients had $A M I$ in the left ventricle. $\mathrm{Cl}$-TEB and $\mathrm{Cl}$-TD were positively correlated $\left(r^{2}=0.75\right)$. Cl-TD was inversely correlated with PCWP $\left(r^{2}=0.22\right)$. TFC was weakly correlated with PCWP $\left(r^{2}=0.26\right)$. Neither Cl-TEB nor Cl-TD had significant correlation with TFC $\left(r^{2}=0.01\right.$ and 0.02 , respectively).

In this study, we confirmed that TEB method might be clinically useful for $\mathrm{Cl}$ measurement in patients with AMI. PCWP was negatively correlated with CI-TD indicating that decreased $\mathrm{Cl}$ in our patient group was not due to systemic dehydration but to decreased cardiac contractility. The weak correlation between PCWP and TFC means that the tendency of pulmonary water retention exists with an increasing PCWP even in patients with normal PCWP. The clinical importance of this increase in TFC is not clear. TFC values had no significant correlation with $\mathrm{Cl}$ values. This means that the degree of pulmonary congestion is unpredictable from $\mathrm{Cl}$ provided that PCWP remains normal. This is consistent with the Forrester classification.

\section{P4 Is the volume of a pleural effusion predictable using the thickness of the pleural lamella measured by sonography as a reference?}

\section{Cardon, N Müller, M Van de Velde, J Ghekiere, K Vandevelde, L Steyaert, E Vandermeersch and M Bourgeois}

Introduction: The aim of this study was to quantify the volume of pleural effusions (PEs) in the critically ill using ultrasound. PE was suggested on the daily postero-anterior chest radiography [1] in the semirecumbent position. All patients with suspected PE were investigated with ultrasound. We hypothesize that there is a strong correlation between the maximal width of the fluid lamella along the lateral chest wall (seen on sonography) and the volume of pleural fluid punctured.

Materials and methods: The study was approved by the hospital Ethics Committee. Eighty-seven consecutive critically ill patients underwent a pleural puncture when ultrasound analysis revealed a lamella of more than $2 \mathrm{~cm}$ [2]. A total of 138 pleural punctures was performed in 87 individuals. Ultrasound was performed using the ACUSON, sequoia 512, with the patient in the semirecumbent position. The deepest possible puncture side in this position was marked for pleural puncture. The PE was gradually drained, $200 \mathrm{ml}$ at a time, until the fluid was completely evacuated. The width of the pre-puncture effusion lamella as measured with ultrasound was compared case by case with the actual punctured volume. Statistical analysis was performed using linear regression analysis and Spearman (rank) correlation coefficient.

Results: Due to technical difficulties or missing data, 7 punctures had to be excluded. 131 punctures (67 on the left hemithorax and 64 on the right) remained for analysis. No complications were encountered as a consequence of the pleural puncture.

The sonographic measurements correlated very well with actual effusion volume on the left $\left(r_{s}=0.83\right)$ and the right

\begin{tabular}{lcccc} 
& $\begin{array}{c}\text { Pleural } \\
\text { thickness } \\
\text { left }(\mathrm{cm})\end{array}$ & $\begin{array}{c}\text { Pleural } \\
\text { volume } \\
\text { left }(\mathrm{ml})\end{array}$ & $\begin{array}{c}\text { Pleural } \\
\text { thickness } \\
\text { right }(\mathrm{cm})\end{array}$ & $\begin{array}{c}\text { Pleural } \\
\text { volume } \\
\text { right }(\mathrm{ml})\end{array}$ \\
\hline Mean & 3.55 & 428.13 & 3.72 & 505.63 \\
Median & 3 & 400 & 3.5 & 410 \\
Std. Deviation & 1.15 & 310.71 & 1.26 & 362.65 \\
Percentile 25 & 2.5 & 150 & 2.9 & 212.5 \\
Percentile 50 & 3 & 400 & 3.5 & 410 \\
Percentile 75 & 4.225 & 600 & 4 & 800 \\
\hline
\end{tabular}



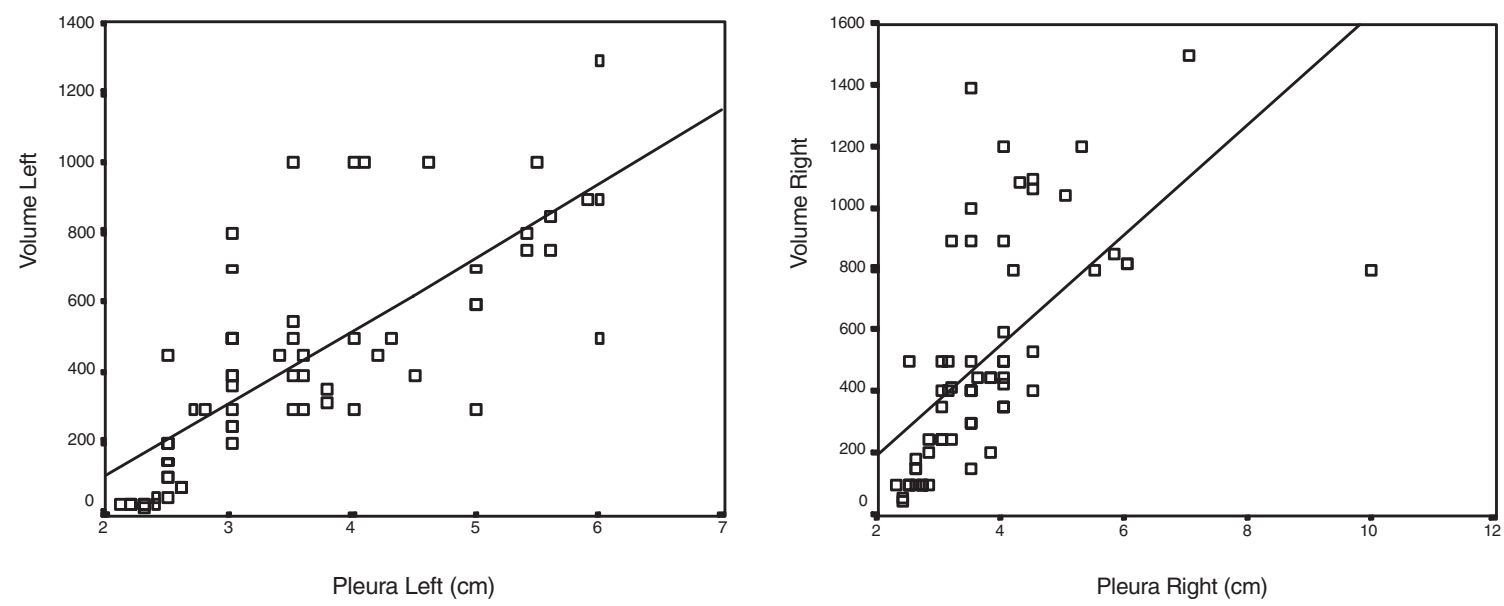

Left: $r s=0.83, P<0.001, y=208.77 \times-317.12$. Right: $r s=0.77, P<0.001, y=178.38 \times-159.7$.

side $\left(r_{s}=0.77\right)$. For both sides the level of statistical significance was taken as $P<0.001$.

The thickness of the fluid lamella was taken as the independent variable, while the actual effusion volume was taken as the dependent variable. The linear sonographic method was represented by the equation $y=208.77 x-317.12$ (left), and $y=178.38 x-159.7$ (right). $Y$ is the predicted effusion volume in milliliters and $x$ is the sonographically measured thickness of the effusion lamella in centimeters. The mean predicted error was $199.7 \mathrm{ml}$ for the left side and $285.3 \mathrm{ml}$ for the right side.
Discussion: There is a strong correlation between the sonographic measurements and the actual effusion volume which was punctured. Unlike the findings of other authors, we were not able to predict the punctured volume, based on the width of the lamella measured by sonography probably due to the wide spread of obtained data (scatter plot).

\section{References:}

1. Müller NL: Imaging of the pleura. Radiology 1993, 186:297-309.

2. Eibenberger KL, Dock WI, Ammann ME, Dorffner R, Hörmann MF, Grabenwöger F: Quantification of pleural effusions: sonography versus radiography. Radiology 1994, 191:681-684.

\section{P5 Evaluation of a new wireless ECG electrode built in a telemeter in critically ill patients}

Y Sato, K Ikegami, F Morimoto, Y Maekawa, T Sekiguchi*, F Matsumura* and H Sakata*

Trauma and Critical Care Center, Koshigaya Hospital, Dokkyo University School of Medicine, 2-1-50 Minami-Koshigaya, Koshigaya, Saitama, Japan; ${ }^{*} R$ and D Center, Nihon Kohden Corporation, Tokyo, Japan

Introduction: Aiming for the improvement of 'spaghetti syndrome' caused by many leadwires for the measurements of biological signals such as electrocardiogram (ECG), BP, $\mathrm{SpO}_{2}, \mathrm{EtCO}_{2}$ and lines for intravenous infusion, we developed the new small wireless ECG electrode (size: $3 \mathrm{~cm} \times 10 \mathrm{~cm})$ consisting of a battery-operated telemeter equipped with two electrodes. The purpose of this study was to evaluate the ECG with the wireless ECG electrode placed on critically ill patients in the emergency room.

Methods: ECGs were recorded with both a wireless ECG electrode placed on the right subclavian area and a conventional three-lead ECG (II) in 30 critically-ill patients whose ECGs were not only of normal rhythm but also arrhythmic. The 12-lead ECG (II) was also recorded. The wireless ECG electrode was also evaluated during cardiopulmonary resuscitation.
Results: The wireless ECG electrode was quickly and easily placed on the patients compared with the conventional ECG electrode. ECGs were recorded very well in all patients utilizing the wireless ECG electrode. It was also possible to detect various arrhythmias such as VPC, SVPC, Af, VF, VT with the wireless ECG electrode as clearly as with the conventional ECG electrode. The influence of body movement was less than for the conventional ECG electrode. The wireless ECG electrode was operative even during DC shock.

Conclusion: It was found that the wireless ECG electrode was superior in its handling, ECG performance, and durability, suggesting its possible contribution toward the improvement of 'spaghetti syndrome' in the emergency room. 


\section{P6 Immediate complications of central venous cannulation in ICU}

AM Varvinski and GP Findlay

Critical Care Directorate, University Hospital of Wales, Heath Park, Cardiff, Wales, UK, CF4 4XW

Introduction: Catheterization of the central veins is one of the most widespread procedures in any Intensive Care Unit (ICU). Despite the availability of different literature on anatomy, techniques and the introduction of special ultrasound devices, complications are frequent. Complication rates range from $7-20 \%$ according to the literature.

Methods: This is a prospective study to assess the complication rate from central venous cannulation in the ICU. A separate registration form for every attempted catheterization was used. Data collected were: patient's demographics, grade of operator, approach used, number of attempts, indication for insertion, use of ultrasound probe, type of catheter and complications.

Results: In the first three months, 82 patients were involved in the study. There were 120 attempted catheterizations and 115 lines were inserted (success rate,
95.8\%). There were 21 complications (complication rate, $17.5 \%)$. They were intra-arterial insertion/puncture 9 $(7.5 \%)$, perivascular insertion $3(2.5 \%)$, pneumothorax 1 $(0.83 \%)$, bleeding requiring blood transfusion $1(0.83 \%)$, arrhythmias requiring intervention $1(0.83 \%)$, surgical emphysema $1(0.83 \%)$, unsuccessful 5 (4.16\%).

Conclusion: The complication rate of central venous catheterization remains high even in experienced hands. Fatal or serious complications are rare. The use of the ultrasound probe may decrease the complication rate even if used for checking the anatomy only. Intra-arterial puncture/insertion, considered by many as a relatively innocent complication, can lead to unnecessary morbidity and even fatal complications, especially in the ITU population with abnormal clotting. Formal teaching should be provided for all new staff in training starting their appointments on the Intensive Care Units.

\section{P7 A comparison of pulmonary artery occlusion pressure (PaOP) measurements using pressure controlled ventilation (PCV) versus airway pressure release ventilation (APRV)}

\section{LJ Kaplan and H Bailey}

Medical College of PA Hospital, Departments of Surgery and Emergency Medicine, Division of Trauma and Critical Care, 3300

Henry Avenue, Philadelphia, PA 19129, USA

Purpose: To determine the optimal time within the APRV phase cycle to accurately measure $\mathrm{PaOP}$.

Methods: Ten consecutive patients with acute lung injury (ALI) managed with PCV and a pulmonary artery catheter (PAC) were studied. Demographic data was recorded. Patients served as their own controls and were ventilated by a Drager Evita 4 Pulmonary Workstation. No patients received paralytics. PCV settings (AC mode) achieved a $\mathrm{pCO}_{2}$ of $35-45$ (torr) and a $\mathrm{pO}_{2}>60$ (torr) on $60 \% \mathrm{O}_{2}$; PEEP was not controlled. Hemodynamic profiles were recorded 30 min after achieving the above $\mathrm{pCO}_{2}$ and $\mathrm{pO}_{2}$ values. Patients were then changed to APRV to achieve the same $\mathrm{pCO}_{2}$ and $\mathrm{pO}_{2}$ values and hemodynamic measurements were repeated at $30 \mathrm{~min}$. All medications were held constant. PaOP tracings $(\mathrm{mmHg})$ were recorded and compared to the downloaded flow-time trace from the ventilator (Evitaview software). The PCV PaOP was recorded at the end of exhalation and served as the standard for comparisons. PaOP was recorded during the APRV phase cycle (positive pressure and release) and compared to the PCV value. Data are shown as means tstandard deviation and were compared using a twotailed paired t-test; significance assumed for $P<0.05$.
Results: Principal diagnoses were trauma (66\%), abdominal sepsis (32\%), and other (2\%). Mean age was $54 \pm 6.2$ years. PCV blood gas values were $\mathrm{pH} 7.34 \pm 0.04, \mathrm{pCO}_{2}$ 39.3 $\pm 3.8, \mathrm{pO}_{2} 77.4 \pm 9.5$. APRV blood gas values were $\mathrm{pH} 7.37 \pm 0.03, \mathrm{pCO}_{2} 35.5 \pm 2.8, \mathrm{pO}_{2} 98 \pm 11,(P<0.05$ vs $\mathrm{PCV})$. The PCV PaOP was $16.3 \pm 3$ on a PEEP of $13.6 \pm 2.2 \mathrm{cmH}_{2} \mathrm{O}$ with a $\mathrm{Cl}$ of $3.2 \pm 0.5 \mathrm{~L} / \mathrm{min} / \mathrm{m}^{2}$ and an $\mathrm{SvO}_{2}$ of $76.8 \pm 4.5 \%$ at a hemoglobin of $9.6 \pm 1.04 \mathrm{gm} \%$. The APRV PaOP during the positive pressure phase was 21.2 \pm 3.3 (initial), $19 \pm 2.5$ (mid), and 20.5 \pm 2.8 (end); $P<0.01$ for all versus PCV. The APRV PaOP during the release phase was $19 \pm 2.7$ (initial, $P<0.05$ ), $17.7 \pm 2.3$ (mid, $P=0.09$ ), and 16.4 \pm 2.6 (end, $P=0.9$ ). Cl was significantly increased at $3.6 \pm 0.4$ ( $P<0.01$ vs $\mathrm{PCV}$ ) while $\mathrm{SvO}_{2}$ was unchanged at $79.1 \pm 4.1$ ( $P>0.05$ vs PCV).

Conclusions: APRV increases the measured $\mathrm{PaOP}$ during the positive pressure phase. PaOP may be reliably measured at the midpoint or end of the release phase of APRV. APRV increases oxygenation and cardiac index compared to PCV in patients with acute lung injury. 


\section{P8 Effective pulmonary capillary pressure during hyperdynamic porcine endotoxemia}

T Handschuh, D Vasilev, M Georgieff, P Radermacher and B Šantak

Department of Anesthesia, University Hospital, D-89073, Ulm, Germany

Introduction: The pulmonary microvascular hydrostatic or effective capillary pressure $\left(\mathrm{PCP}_{\text {eff }}\right)$ is the principal determinant of the rate of edema formation $[1,2]$. Since scarce data are available only on the natural development of $\mathrm{PCP}_{\text {eff, }}$ independently from vasoactive treatment, we investigated the time course of both $\mathrm{PCP}_{\text {eff }}$ and the longitudinal distribution of pulmonary vascular resistance during long-term hyperdynamic porcine endotoxemia.

Methods: Over $9 \mathrm{~h}$ anesthetized and ventilated pigs received continuous intravenous endotoxin $(E T X, n=7)$ or placebo (Sham, $n=5$ ). Hydroxyethylstarch was infused to maintain MAP $>65 \mathrm{mmHg}$ together with a sustained 30-50\% increase in cardiac output. Mean (MPAP) and pulmonary artery occluded (PAOP) pressures were recorded before and 3,6 , and $9 \mathrm{~h}$ after the start of ETX. After signal filtering via a low-pass filter, $\mathrm{PCP}_{\text {eff }}$ was derived from a bi-exponential fit to the post-occlusion pressure decay curve, back-extrapolating the slower part to the instant of occlusion determined by simultaneously recording the balloon pressure [1]. The contribution of the venous resistance to the pressure drop over the pulmonary vascular bed was calculated as $\mathrm{PVR}_{\mathrm{ven}}=\left(\mathrm{PCP}_{\mathrm{eff}^{-}}\right.$ PAOP)/(MPAP-PAOP) [1].

Results: See Table.

Conclusion: Early endotoxemia results in a pronounced increase of $\mathrm{PCP}_{\text {eff }}$ both due to increased downstream (i.e. left atrial) pressure as well as increased contribution of the postcapillary pressure drop. Hypoxia and/or early mediator release (i.e. thromboxane) probably assume importance in this context [1]. The later normalization of both $\mathrm{PCP}_{\text {eff }}$ and $\mathrm{PVR}_{\mathrm{ven}}$ may be due to increased $\mathrm{NO}$ formation [3].

Acknowledgement: D Vasilev is supported by the Deutscher Akademischer Austauschdienst.

References:

1. Cope et al.: Critical Care Med 1992, 20:1043.

2. Grimbert: Eur Respir J 1988, 1:297.

3. Hinder et al.: J Appl Physiol 1997, 83:1941.

\begin{tabular}{|c|c|c|c|c|c|}
\hline & & Before ETX & 3 h ETX & 6 h ETX & 9 h ETX \\
\hline MPAP & ETX & $22(18 / 23)$ & 47 (42/52)\#* & $44(41 / 52) \#^{*}$ & $34(32 / 35) \#^{*}$ \\
\hline $\mathrm{mmHg}$ & Sham & $19(17 / 22)$ & $19(18 / 21)$ & $22(20 / 25)$ & $22(21 / 23)$ \\
\hline PAOP & ETX & $7(5 / 10)$ & $11(10 / 13) \#^{*}$ & $9(7 / 12)$ & $8(7 / 11)$ \\
\hline $\mathrm{mmHg}$ & Sham & $6(4 / 7)$ & $6(5 / 7)$ & $5(4 / 5)$ & $6(5 / 7)$ \\
\hline $\mathrm{PCP}_{\text {eff }}$ & ETX & $10(8 / 11)$ & $24(20 / 26) \# *$ & $17(11 / 24)^{\star}$ & $14(12 / 16)$ \\
\hline $\mathrm{mmHg}$ & Sham & $8(6 / 9)$ & $7(6 / 7)$ & $8(6 / 8)$ & $9(7 / 10)$ \\
\hline $\mathrm{PVR}_{\text {ven }}$ & ETX & $23(21 / 27)$ & $37(34 / 39)^{\star}$ & $24(23 / 27)$ & $22(19 / 26)$ \\
\hline$\%$ & Sham & $19(18 / 22)$ & $15(11 / 18)$ & $16(15 / 19)$ & $16(14 / 20)$ \\
\hline
\end{tabular}

Median (25/75\%), \# $P<0.05$ vs Oh (Friedman-Anova); ${ }^{\star} P<0.05$ ETX vs Sham (Mann-Whitney)

\section{P9 A comparison between oesophageal Doppler and continuous thermodilution for the} measurement of cardiac output in critically ill patients

JR Wilkinson, C Ruff, SK Patel, RM Venn, A Rhodes and ED Bennett

Department of Intensive Care, St George's Hospital, Tooting, London, SW17 OQT, UK

Introduction: There have been concerns raised over the safety of pulmonary artery (PA) catheters. Previous studies have shown oesophageal Doppler (OD) monitoring provides a reliable, easy alternative method of assessing cardiac output (CO) in an ICU setting.

Methods: After ethical approval we studied 12 ICU patients who required PA catheters for monitoring of therapy. A continuous cardiac output (CCO) PA catheter (Vigilance monitor, Baxter) and OD probe (CardioQ monitor, Deltex), were inserted in each patient. Insertion time and complications were noted for both techniques. Measurements of $\mathrm{CO}$ by thermodilution and OD were recorded. Data was analysed using regression analysis and Bland-Altman for the difference between the two techniques.

Results: Twelve patients were analysed with a total of 128 pairs of cardiac output measurements. There were four complications due to PA catheters and none due to OD. Mean insertion time was 52.9 min for PA catheter and $5.8 \mathrm{~min}$ for OD $(P<0.0001)$. Thermodilution cardiac outputs were obtained between 2.9 and $15.5 \mathrm{~L} / \mathrm{min}$. Pearson's correlation coefficient ( $r$ ) for PA catheter vs OD was $0.689(P<0.0001)$. The mean difference in $\mathrm{CO}$ between 
the two methods was $-0.17 \mathrm{~L} / \mathrm{min}$ with limits of agreement of -4.016 to +3.676 .

Discussion: To our knowledge, this is the first study to compare continuous cardiac output by thermodilution with cardiac output by OD (Cardio probes are faster to insert, easier to use and safer. However, our study shows that there are major differences in the cardiac output values obtained by the two techniques.

\section{P10 Comparison of pulmonary arterial and arterial trans-cardiopulmonary thermodilution cardiac output in porcine septic shock}

B Vangerow, M Cobas Meyer, J Ahrens, T Schuerholz, G Marx, M Moeller, M Leuwer and H Rueckoldt Department of Anaesthesiology, Hannover Medical School, D-30625, Hannover, Germany

Introduction: Despite its invasiveness and inherent risks, the pulmonary artery catheter (PAC) is still regarded as the clinical standard for cardiac output $(\mathrm{CO})$ determination. Arterial trans-cardiopulmonary thermodilution is a less invasive method (PAC not necessary) for CO monitoring. The aim of this study was to compare arterial trans-cardiopulmonary thermodilution to conventional pulmonary arterial thermodilution for $\mathrm{CO}$ determination during substantial hemodynamic variations in a sepsis model.

Methods: In a prospective study 24 anaesthetized, mechanically ventilated pigs $(19.7 \pm 1.6 \mathrm{~kg})$ with peritonitis-induced septic shock were investigated. Cardiac output was determined using a $7.5 \mathrm{~F}$ thermodilution catheter placed in the pulmonary artery and a $4 \mathrm{~F}$ thermistor tipped catheter (Pulsion Medical Systems, Germany) inserted into the right carotid. Nine sets of corresponding $\mathrm{CO}$ determinations were obtained during a period of $8 \mathrm{~h}$ in each animal, all measurements were performed in triplicate. Data were analyzed using Bland-Altman analyses, linear regression and correlation.
Results: During the period from induction of peritonitis to profound septic shock, major variations in heart rate (range: $48-310$ beats $/ \mathrm{min}$ ) and systemic vascular resistance (range: 7400-1340 dyne $\times \sec \times \mathrm{cm}^{-5}$ ) were observed. 196 sets of CO determinations were yielded with a mean $\mathrm{CO}$ measured by pulmonary arterial thermodilution (PATD) of 2.0 $\pm 0.7 \mathrm{~L} / \mathrm{min}$ (range: $0.7-5.2 \mathrm{~L} / \mathrm{min}$ ). Mean $\mathrm{CO}$ measured by arterial trans-cardiopulmonary thermodilution (ATPTD) was $2.17 \pm 0.6 \mathrm{~L} / \mathrm{min}$ (range: 1.0-4.4 L/min). Linear regression equation was: ATPTD $=0.711 \times$ PATD $+0.82 ; r^{2}=0.68$. The mean bias was $0.17 \mathrm{~L} / \mathrm{min}$ (95\% confidence interval: $0.125-0.215$ $\mathrm{L} / \mathrm{min}$ ), with limits of agreement of -0.61 to $0.95 \mathrm{~L} / \mathrm{min}$ and a precision of $0.34 \mathrm{~L} / \mathrm{min}$.

Conclusion: Arterial trans-cardiopulmonary thermodilution $\mathrm{CO}$ correlates acceptably with pulmonary arterial thermodilution $\mathrm{CO}$ even during pronounced hemodynamic instability.

\section{P11 Comparison of pressure-related performance data with data measured by thermodilution in heart} failure and sepsis

\section{Engelmann, J Otto and HE Kunig*}

University of Leipzig, Center of Internal Medicine, Department of Intensive Care, Philipp-Rosenthal- Straße 27a, 04103 Leipzig, Germany; * P.O. Box 192, Saltsburg, PA 15681-0192, USA

Introduction: The performance diagrams evaluate the cardiocirculatory function by means of blood pressure, pulse rate and body surface area (BSA). The basics are represented by Kunig et al. in this symposium. The performance data predict nonsurvival with a sensitivity of $94 \%$ and a specificity of $84 \%$.

Aim of study: Comparison of hemodynamic data measured by thermodilution with pressure-related performance diagrams and their use in differentiation of critical cardiocirculatory states.

Method: On-line measured performance data are compared with discontinuously determined thermodilution data in patients with heart failure $\left(\mathrm{Cl}<2.5 \mathrm{l} / \mathrm{min} / \mathrm{m}^{2}\right.$; $\mathrm{PCW} P>20 \mathrm{mmHg})$ and sepsis $\left(\mathrm{Cl}>4.5 \mathrm{l} / \mathrm{min} / \mathrm{m}^{2} ; \mathrm{PCWP}\right.$ $>16 \mathrm{mmHg}$ ).
Results: In 9 patients with cardiac failure (Cl 1.94 \pm 0.44 $1 / \mathrm{min} / \mathrm{m}^{2}$; PCWP $23.3 \pm 5.5 \mathrm{mmHg}$ ) the efficiency (EF[P]) declines to $0.27 \pm 0.03$, the diastolic blood pressure (DBP) and systolic blood pressure (SBP) range slightly above the basal values $\left(\mathrm{DBP}^{\star} 2.64 \pm 0.58\right.$; $\left.\mathrm{SBP}^{\star} 3.61 \pm 0.73\right)$ and the pulse pressure rate $\left(\mathrm{PP}^{*}\right)$ below the critical border of 1

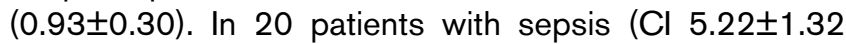
$\mathrm{l} / \mathrm{min} / \mathrm{m}^{2}$; PCWP $19.4 \pm 4.4 \mathrm{mmHg}$ ) the efficiency is normal $(0.48 \pm 0.07)$, while $\mathrm{SBP}^{*}$ and $\mathrm{DBP}^{*}$ are remarkably increased (5.16 \pm 1.23 and $2.69 \pm 0.85)$. The $\mathrm{PP}^{*}$ ranges is $2.52 \pm 0.61$. The differences in $\mathrm{Cl}, \mathrm{EF}(\mathrm{P}), \mathrm{PP}^{\star}$ and $\mathrm{SBP}^{*}$ are strongly significant $(P<0.01)$ in sepsis compared with heart failure.

Conclusions: (1)The pressure-related performance data show changes in case of abnormal deviations of volumetrically determined hemodynamic parameters.(2) A differenti- 
ation of hemodynamic parameters between sepsis and severe heart failure succeeds by means of $\mathrm{Cl}, \mathrm{EF}(\mathrm{P}), \mathrm{PP}^{*}$ and $\mathrm{SBP}^{\star}$. (3) A remarkable deviation of performance parameters from basal values signals a cardiocirculatory deterioration, which should be analyzed by conventionally measured hemodynamic parameters at present.

\title{
P12 Compliance or failure and improvement or deterioration diagnosis of patients from performance diagrams
}

\author{
HE Kunig, SV Kunig, L Engelmann*, U Pilz*, J Otto*, L Mende*, E Huettemann†', TJ Gallagher ${ }^{\ddagger}$, J Elledge-Nauman, \\ JK Stene $\$$, P Tassani”, U Jaenicke ${ }^{\star *}$, H Patrick $^{\dagger+}$ and MR Pinsky ${ }^{\ddagger \ddagger}$ \\ Dept. Bioengineering, University of Washington, Seattle, USA; ${ }^{*}$ Dept. Int. Care Med. University of Leipzig; ${ }^{\dagger}$ Dept. Crit. Care \\ Med., University of Jena; ${ }^{\ddagger}$ Dept. Crit. Care Med., University of Florida, USA; §Dept. Crit. Care Med., Pennsylvania State \\ University, USA; \#German Heart Center, Munich; ${ }^{*}$ Dept. Anesth., University of Munich, Germany; ${ }^{+\dagger}$ Dept. Crit. Care Med., \\ Jefferson Med. College, Philadelphia, USA; ${ }^{\ddagger \neq}$ Dep. Crit. Care Med., University of Pittsburgh USA.
}

Introduction: Blood pressure and heart rate data displayed in a performance diagram (PD) may diagnose accurately compliance or failure and improvement or deterioration while traditional hemodynamics would indicate a patient to be stable.

Materials and methods: A PD plots the parameters pressure efficiency $(E F[P])$ versus time in an upper graph and arterial pulse pressure $\left(\mathrm{PP}^{\star}\right)$, systolic pressure $\left(\mathrm{SBP}^{\star}\right)$, and diastolic pressure $\left(\mathrm{DBP}^{\star}\right)$ versus time in a lower graph. $E F(P)=P P^{*} / S B P^{*}$ in analogy to the volume ejection fraction $E F(V)=S V / E D V$, where $S V=$ stroke volume and $E D V=e n d-d i a s t o l i c$ volume. The asterisk $\left(^{*}\right)$ indicates conversion of events per beat into events per time and standardization to body surface area (BSA), f. e., $\mathrm{PP}^{*}=(\mathrm{PPxHR}) / \mathrm{BSA}$, analogous to the conversion of $\mathrm{SV}$ to cardiac index $(\mathrm{Cl})$, where $\mathrm{Cl}=\left(\mathrm{SV}^{\star} \mathrm{HR}\right) / \mathrm{BSA}$. PDs suggest: (1) compliance when all parameters equal or exceed normal values $(N),(2)$ failure without immediate danger of death when normal values of $\mathrm{EF}(\mathrm{P})$, or $\mathrm{SBP}^{\star}$, or $\mathrm{DBP}^{\star}$ are not maintained, (3) failure with immediate danger of death (critical illness), when normal $\mathrm{PP}^{*}$ is not maintained, (4) deterioration or improvement when the trend of two successive measurements departs from, or points towards, the normal values. Data from 213 patients were retrospectively analyzed using standard statistical methods including sensitivity and specificity determinations, binomial scoring, and t-testing.

Results: Based on statistical analysis, PDs predicted compliance and failure with a sensitivity of $94 \%$ and a specificity of $84 \%$. PDs also predicted improvement and deterioration at $P<0.01$. As illustrated in the figures, traditional hemodynamics diagnoses a patient as stable as late as 10 min before the occurrence of flash edema. The PD diagnoses a failing and deteriorating patient as early as $2.5 \mathrm{~h}$ prior to occurrence of flash edema and diagnoses the patient as critically ill 25 min prior to the occurrence of the flash edema.

Discussion: This study suggests utility of PDs in accurately diagnosing compliance or failure which would allow early intervention and monitoring of the effects of intervention in real time as compared to traditional hemodynamic evaluation.

\section{Traditional Hemodynamics}
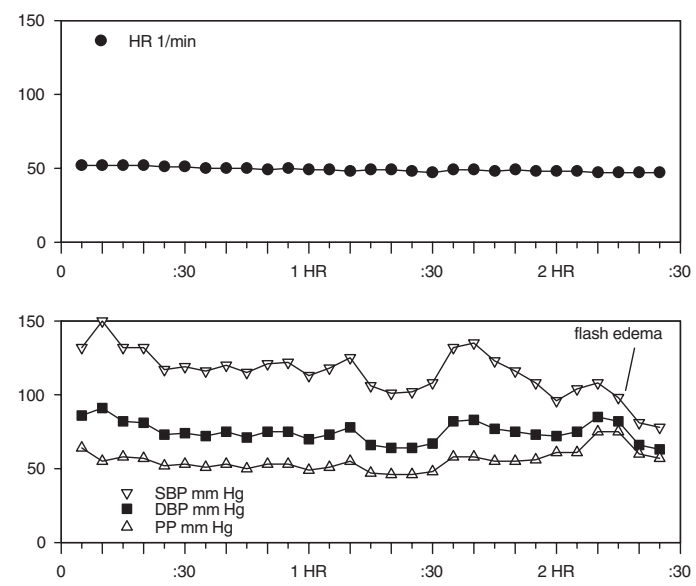

Performance Diagram

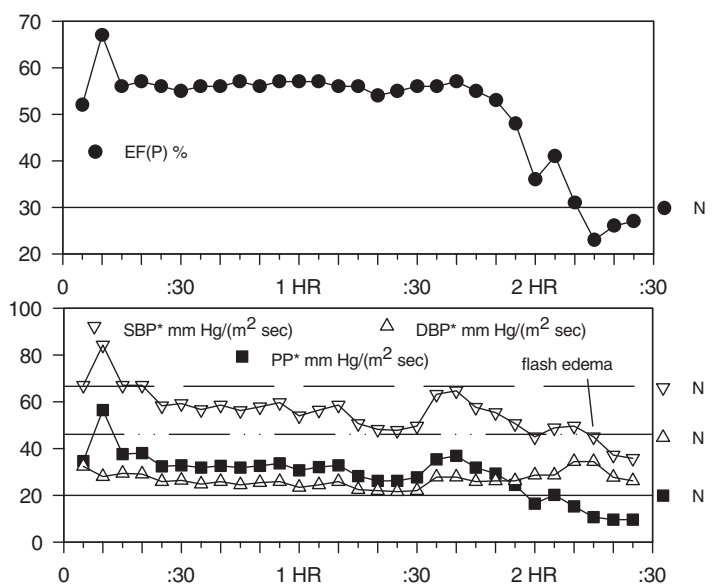




\section{P13 Cl/SVRI relationship during different phases of inflammatory response}

\section{R Kula, P Sklienka, I Petrašovicová, L Kolár and J Jahoda}

Department of Anaesthesia and Intensive Care, Faculty Hospital, Ostrava, Czech Republic

Introduction: High cardiac index $(\mathrm{Cl})$ and low systemic vascular resistance (SVRI) are frequently observed in septic patients and are essential in consideration of further therapeutic interventions. As these findings could be observed even in healthy individuals (i.e. during hard exercise) we decided to analyse the relationship between $\mathrm{Cl}$ and SVRI in patients without apparent clinical signs of the inflammatory response and in patients with the different phase of the inflammatory response.

Methods: Thirty-one critically ill patients with pulmonary artery catheter inserted were included in this prospective study (average age $48 \pm 16$ years, average sum of SOFA score 2.4 \pm 1.36 , etiology: $60 \%$ traumatic patients), all of them with apparent clinical signs of the inflammatory response (heart rate $>90 \mathrm{bpm}, \mathrm{WBC}>12000$ or $<4000$, $\mathrm{BT}>38^{\circ} \mathrm{C}$ or $\left.<36^{\circ} \mathrm{C}, \mathrm{CRP}>50 \mathrm{mg} / \mathrm{l}\right)$ with microbiologically confirmed infectious etiology. In accordance with our findings (published in Int Care Med 1997, 23(Suppl.1.):S72) we identified 16 periods of generalization of the inflammatory response (fall in platelet count, antithrombine-III activity and serum albumin, positive fluid balance, hemodynamic instability). Within these periods, 101 hemodynamic measurements were performed, measured parameters are shown in Fig. 1. In periods without symptoms of generalized inflammatory response we performed 115 hemodynamic measurements, results of which are shown in Fig. 2. 130 hemodynamic measurements in patients without apparent clinical signs of the inflammatory response (25 patients, age $51 \pm 17$ years, pulmonary artery catheter inserted in setting of preoperative hemodynamics optimization) we used as the control group. Results are shown in Fig. 3.

\section{Results: See Figures}

Conclusion: The relationship between $\mathrm{Cl}$ and SVRI shows a different course in the phase of the generalized inflammatory response compared to the phase of the localized inflammatory response. The course of the relationship between $\mathrm{Cl}$ and SVRI in patients without inflammatory response is nearly identical to that in patients with symptoms of the localized inflammatory response. On the basis of these findings, we presume that assessment of the relationship between $\mathrm{Cl}$ and SVRI (but not isolated $\mathrm{Cl}$ and/or SVRI) is needed for accurate therapeutic decision making.

\section{Reference:}

Kula et al.: Int Care Med 1997, 23(Suppl.1):S74.
Figure 1

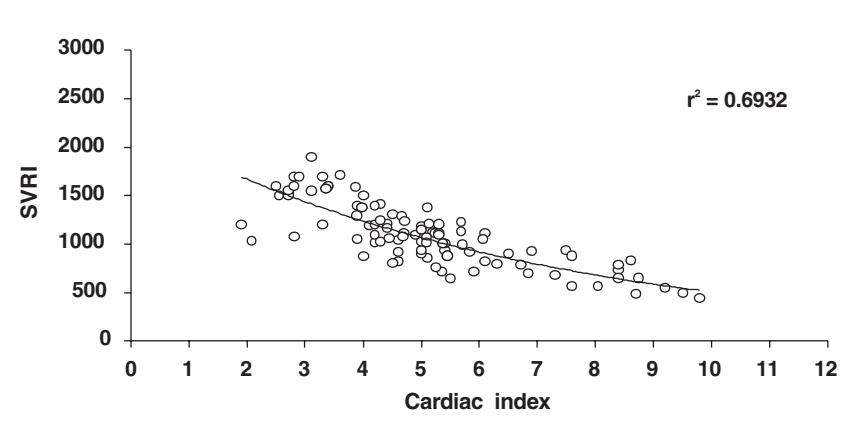

$\mathrm{Cl} / \mathrm{SVRI}$ relationship during period of the generalized inflammatory response

Figure 2

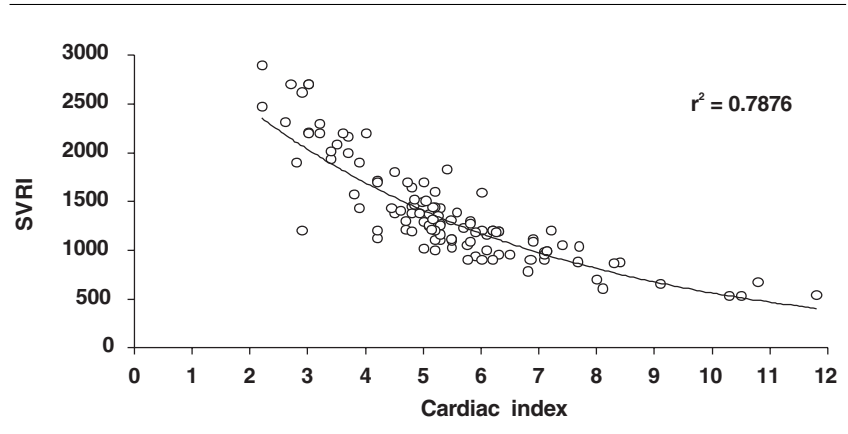

$\mathrm{CI}$ SVRI relationship during period of the localized inflammatory response

Figure 3

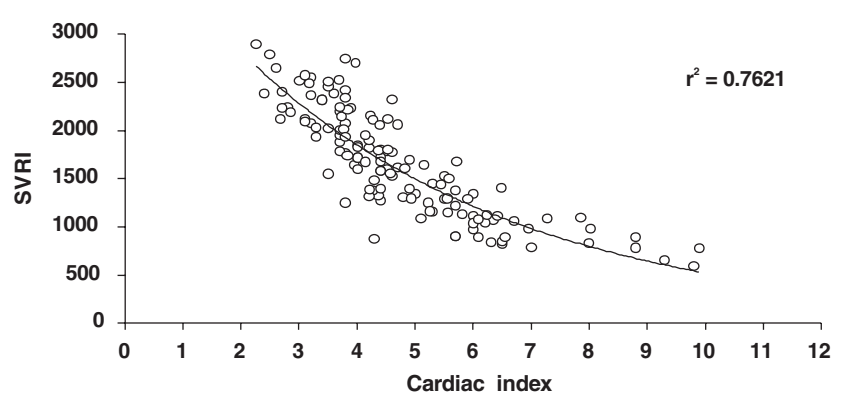

$\mathrm{Cl} / \mathrm{SVRI}$ relationship in patients without the inflammatory response 


\section{P14 Would the blood volume analyzer-100 (BVA-100) be an alternative to pulmonary artery catheter (PAC) in critically ill patients}

S Alrawi, W Cholewczynski, R Raju, A Acinapura and J Cunningham

Department of Surgery, Trauma office, Lutheran Medical Center, 150 55th Street, Brooklyn, New York, NY 11220, USA

Background: Measurements of urine output, mean arterial pressure (MAP), heart rate (HR), pulmonary wedge pressure $(\mathrm{PWP})$ and cardiac index $(\mathrm{Cl})$ reflect the status of central blood volume including heart, brain, and lungs but do not reflect the status of peripheral blood. The accurate measurement of total blood volume provided by a certain noninvasive technique compared to a 'guesstimate' given by hematocrit reading or by PAC with all its possible complications could mean the difference between life and death. The standard tests-hemoglobin and hematocrit-do not provide an accurate measure of blood volume in sudden acute blood loss situations.

Material and methods: Twenty-five critically ill patients in the intensive care unit (ICU; surgical and medical) with PAC and fourteen normal persons (control) were evaluated after IRB approval for total blood volume measurement (BVM) by BVA-100. The instrument is used in conjunction with a special patented syringe, which delivers a precise, quantitative injection of Albumin I-131 isotope into the patient. Six consecutive blood samples were then taken. All
ICU patients were classified according to the APACHE II severity of disease classification system, PAC parameters were considered and fluid balance, ventilatory settings, vasopressors and treatment were evaluated. Serum lactate, arterial blood gas and mixed venous blood gas were determined. All these parameters were correlated with total blood, red cell and plasma volume.

Results: We find no significant correlation between blood volume (BV), pulmonary capillary wedge pressure (PCWP) and central venous pressure (CVP) readings. There is also no correlation between (BV) and the other variables in the study including base excess from arterial blood gas, mixed venous blood gas saturation, cardiac output, cardiac index, systemic vascular resistance, serum lactate and oxygen delivery $\left(\mathrm{A}-\mathrm{VO}_{2}\right)$.

Conclusion: Clinical decision-making in the management of critically ill patients regarding blood volume status should be measured by direct blood volume analysis rather than by the indirect parameters of PCWP and CVP.

\section{P15 Sepsis increases accumulation of cell-free hemoglobin in intima of submucosal arterioles in rats}

AW Sielenkämper, K Eicker, H Kloppenborg, R Dreyer*, WJ Sibbald ${ }^{\dagger}$ and H Van Aken

Department. of Anesthesiology and Intensive Care Medicine, *Department of Pathology, University of Münster, 48129 Münster, Germany; ${ }^{\dagger}$ AC Burton Vascular Biology Laboratory, University of Western Ontario, London, Canada

Introduction: It was reported from in vitro studies that interruption of the endothelial barrier increases endothelial permeability for cell-free hemoglobin solutions[1]. We studied the effects of sepsis on the abluminal distribution of intravenously infused cell-free hemoglobin (DCLHb, Baxter Corp.) in submucosa of rat ileum.

Methods: Rats were randomized to sham laparotomy $(n=6)$ or cecal ligation and perforation (CLP, $n=7)$ to induce sepsis. Twenty-four $\mathrm{h}$ later, $300 \mathrm{mg}$ of DCLHb labeled with Evans-Blue dye was infused. The laparotomy was reopened, and material was obtained from the ileum 90 min after DCLHb infusion for histological examination. Using fluorescence microscopy, the concentration of cellfree hemoglobin in intima and smooth muscle of submucosal arterioles and in submucosal tissue was graded by two blinded investigators using a score $(0$, no; 1 , minor; 2 , moderate; 3 , strong; 4, very strong fluorescence). Additional studies $(n=3)$ were performed to exclude that the tracer alone accounted for the observed effects.
Results: DCLHb fluorescence scores were low in submucosal tissue and in smooth muscle of arterioles for both groups (all $<0.75$ points.). In the intima of arterioles, considerable fluorescence was observed, with scores increased in the septic group as compared to the sham group (3.2 \pm 0.5 vs $1.4 \pm 0.4$ points, mean $\pm S E M, P<0.05$ ). Infusion of Evans-Blue dye alone resulted in scores $<0.75$ points in all tissues.

Conclusion: Sepsis increased the accumulation of cellfree hemoglobin in the intima of submucosal arterioles. This finding may be relevant for the previously documented effects of cell-free hemoglobin to modify perfusion pressure and microvascular blood flow in sepsis [2].

Acknowledgement: This work was supported by Baxter Healthcare Corp., USA.

References:

1. Nakai et al.: J Lab Clin Med 1998, 132:313-319.

2. Sielenkämper et al.: Crit Care Med 2000, in press. 


\section{P16 Diaspirin cross-linked hemoglobin (DCLHb) ensures tissue oxygenation during hemodilution} below the critical hematocrit

G Kemming*, F Meisner, O Habler*, M Kleen*, J Tillmanns, J Hutter, A Pape, J Meier, C Wojtczyk, D Bottino and K Messmer Institute for Surgical Research and ${ }^{*}$ Clinic for Anesthesiology, Klinikum Großhadern, Marchioninistrasse 15, Ludwig-MaximiliansUniversity, D-81377 Munich, Germany

Background: Normovolemic hemodilution (HD) is an effective blood sparing strategy in the perioperative setting. Despite physiologic compensation for reduced hematocrit $(\mathrm{Hct})$ and $\mathrm{O}_{2}$ content $\left(\mathrm{CaO}_{2}\right)$, tolerance to HD is limited. At a Hct called 'critical' $\left(\mathrm{Hct}_{\text {crit }}\right)$, tissue hypoxia occurs.

Objective: To assess, whether HD can be extended below $\mathrm{Hct}_{\text {crit }}$ when using an hemoglobin-based $\mathrm{O}_{2}$ carrier as diluent.

Methods: Twelve anesthetized, mechanically-ventilated pigs were hemodiluted by $1: 1$ exchange of blood with either $10 \%$ DCLHb (DCLHb, $n=6$ ) or $8 \%$ albumin (HSA, $n=6$ ) oncotically matched to DCLHb (diluents were provided by Baxter Healthcare Inc., Boulder, CO). In both groups, measurements were performed at baseline and after HD to (1) preset Hct-values of $15 \%, 8 \%, 4 \% 2 \%, 1 \%$ or (2) until the individual $\mathrm{Hct}_{\text {crit }}$, defined by the onset of myocardial ischemia (ST-segment depression), was reached. Total body $\mathrm{O}_{2}$-delivery and $\mathrm{O}_{2}$-uptake $\left(\mathrm{DO}_{2} \mathrm{I}, \mathrm{VO}_{2} \mathrm{l}\right)$, as well as local tissue $\mathrm{O}_{2}$ partial pressure $\left(\mathrm{tPO}_{2}, \mathrm{MDO}-\right.$ Electrode, Eschweiler, Kiel, Germany) on the surface of liver and skeletal muscle were assessed during every measurement (Median \pm [Q1-Q3]/2; rANOVA, MWU-test $P<0.05$ ).

Results: In the HSA-group, $\mathrm{Hct}_{\text {crit }}$ was $6.1 \pm 0.9 \%$, at which all animals died. In the DCLHb-group, Hct $_{\text {crit }}$ was not reached despite hemodilution to Hct 1.2\%. At Hct $4 \%$ in the DCLHb-group, all parameters $\left(\mathrm{CaO}_{2}, \mathrm{DO}_{2} \mathrm{I}, \mathrm{tPO}_{2}\right.$ : $P<0.05$ vs $\mathrm{HSA} ; \mathrm{VO}_{2}$ I n.s.) were less altered than at $\mathrm{Hct}_{\text {crit }}$ in the HSA-group.

Conclusion: During HD with DCLHb, O2-transport and tissue oxygenation were fully preserved at Hct $4 \%$, while all HSA-animals had died at Hct $6.1 \%$. The difference is due to the $\mathrm{O}_{2}$ transporting capacity of DCLHb.

\section{References:}

Anesth Analg 1997, 85:953-958.

Adv Microcirc 1972, 4:1-77.

Anesth Analg 1992, 75:818-821.

\section{P17 Decrease of red blood cell sialic acid membrane content in septic patients}

\section{K Zouaoui Boudjeltia*, M Piagnerelli†, P Piro*, D Bastin*, E Carlier ${ }^{\dagger}$, P Lejeune ${ }^{\dagger}$ and M Vanhaeverbeek*}

${ }^{*}$ Experimental Medicine Laboratory and ${ }^{\dagger}$ Department of Intensive Care, A. Vésale Hospital, ULB, 6110, Montigny-le-Tilleul, Belgium

Background: Septic shock is a complex physiopathologic model with alterations in the microcirculation, occurring together with changes in biochemical and physiologic characteristics of the blood. Red blood cells (RBCs) of septic patients are characterized by a decreased deformability and an increased aggregatability. These events are also noted in RBCs of diabetic patients secondary to a reduction in sialic acid (SA) RBC membrane content.

Objective: To study the SA RBC membrane content in a septic population.

Methods: We studied blood samples in ICU patients without documented sepsis ( $n=20$, age $69 \pm 12$ years) and with documented sepsis ( $n=20$, age $65 \pm 16$ years).
SA was measured on isolated RBC membrane protein by a fluorimetric technique in high precision liquid chromatography (HPLC; Anumula).

Results: We observed a significant decrease in SA in septic patients compared with nonseptic patients: SA $1.94 \pm 0.79 \mu \mathrm{g} / 100 \mu \mathrm{g}$ protein and SA: $2.2 \pm 0.39 \mu \mathrm{g} / 100$ $\mu \mathrm{g}$ protein $(P=0.013)$, respectively for septic and nonseptic patients.

Conclusions: RBC of septic patients are characterized by a decrease in SA membrane content as described in diabetic patients. These data highlight another possible biochemical modification of RBC in sepsis.

\section{P18 Preload assessment in septic shock}

A Donati, G Conti, S Loggi, S Falcetta, E Adrario, P Pelaia and P Pietropaoli

Istituto delle Emergenze Medico-Chirurgiche, Università di Ancona, Ospedale Regionale Torrette, via Conca, 60020 Torrette (Ancona), Italy

Background: The accuracy of intra thoracic blood volume (ITBV) as a preload index, instead of central venous pressure and wedge pressure, has been demonstrated by Lichtwark-
Ashoff in mechanically-ventilated patients with acute respiratory failure $[1,2]$. The aim of our work was: (1) to verify ITBV as a preload index in patients suffering from septic shock 
and (2) to relate measured data (CVP, WP, ITBV, extra vascular lung water [EVLW]), with $\mathrm{PaO}_{2} / \mathrm{FiO}_{2}$ ratio.

Methods: 15 patients suffering from septic shock were studied. Admission criterion was septic shock refractory to standard therapy with cathecolamines. All patients were monitored with a Swan-Ganz catheter and Cold system (Pulsion, Münich) and received a bolus of methylene blue $(\mathrm{MB})$ at the rate of $3 \mathrm{mg} / \mathrm{Kg}$. Hemodynamic and oxyphoretic data were measured before MB infusion, and $20 \mathrm{~min}, 1 \mathrm{~h}$ and $2 \mathrm{~h}$ after the end of MB infusion, so that 4 sets of data for each patient were obtained to give a total of 60 data points.

Results: There is a significant correlation between ITBV and stroke volume (SV) and between ITBV and cardiac index $(\mathrm{Cl})$. There is also a correlation between ITBV and EVLW. No correlation was found between WP and SV and $\mathrm{Cl}$. Then we plotted WP vs the $\mathrm{PaO}_{2} / \mathrm{FiO}_{2}$ ratio and found that when the $\mathrm{PaO}_{2} / \mathrm{FiO}_{2}$ ratio was $<200$ there was no difference in EVLW between patients with WP $\leq 16$ $\mathrm{mmHg}$ and patients with WP $>16 \mathrm{mmHg}$. On the contrary, plotting ITBV vs $\mathrm{PaO}_{2} / \mathrm{FiO}_{2}$, with $\mathrm{PaO}_{2} / \mathrm{FiO}_{2}$ ratio $>200$, EVLW was very significantly higher $(P<0.001)$ if ITBV was $>1100 \mathrm{ml} / \mathrm{mq}$, than if it was $\leq 1100 \mathrm{ml} / \mathrm{mq}$.

Conclusions: In septic patients, when a respiratory failure happens, ITBV can be normal or high (>1100 ml/mq). A high ITBV is related to a high EVLW, while a high EVLW with a normal ITBV means a normal preload. In this case, it is not useful to decrease preload to increase the $\mathrm{PaO}_{2} / \mathrm{FiO}_{2}$ ratio. ITBV together with $\mathrm{PaO}_{2} / \mathrm{FiO}_{2}$ ratio is useful to optimize hemodynamic therapy during respiratory failure in septic patients and allows us to identify the patients that need diuretic therapy.

\section{References:}

1. Lichtwark-Ashoff $\mathrm{M}$ et al:: Intensive Care Med 1992, 18:142-147.

2. Lichtwark-Ashoff $M$ et al.: J Crit Care 1996, 11:180-188.

P19 The clinical relevance of the fluid balance in critically ill patients

\section{Petrašovicová, P Sklienka, L Kolár, J Jahoda and R Kula}

Department of Anaesthesia and Intensive Care, Faculty Hospital, Ostrava, Czech Republic

Introduction: Fluid management based on the assessment of heart filling pressures (CVP, PCWP) to reach hemodynamic stability is an important point in management of the critically ill patient. In the case of impaired permeability induced by inflammation, this strategy usually results in excessive interstitial fluid sequestration. In this situation, consequent hemodynamic stability is often at the expense of positive fluid balance and oedema formation, which can cause tissue oxygenation to deteriorate resulting in organ failure progression. The aim of our study was to analyse the relationship between fluid intake and fluid balance together with development of organ dysfunction in surviving and nonsurviving critically ill patients over the course of ten consecutive days.

Materials and methods: 117 critically ill patients admitted to our department from 1996 to 1998 were included in this retrospective study. Criterion for inclusion was hospitalisation longer than 3 days. Patients were divided in two groups: survivors ( $n=70$, average age $49 \pm 16$ years, $54 \%$ multiple trauma, 24\% elective surgery, 26\% medical, average duration of ICU stay $15 \pm 8$ days) and nonsurvivors ( $n=47$, average age $51 \pm 17$ years., $48 \%$ multiple trauma., $20 \%$ elective surgery, $32 \%$ medical, average duration of ICU stay $17 \pm 11$ days). In both groups, fluid intake and fluid balance were evaluated during a ten day interval (from 3rd until 13th day of ICU stay, Fig. 1, Fig. 2) together with intensity of inflammatory response (average CRP value during whole period of evaluation) and organ dysfunction development (sum of SOFA score on 3rd and 13th day of ICU stay, Fig. 3).
Results: See Figures.

Conclusion: In the nonsurvivors group a much more positive fluid balance was found within the interval from 3rd until 13th day of ICU stay, together with increasing extent of the organ dysfunction. The stronger correlation between fluid intake and fluid balance in nonsurviving patients compared to survivors (Fig. 1) points to the need for careful fluid management in critically ill patients, especially in those with an intensive inflammatory response.

\section{Figure 1}

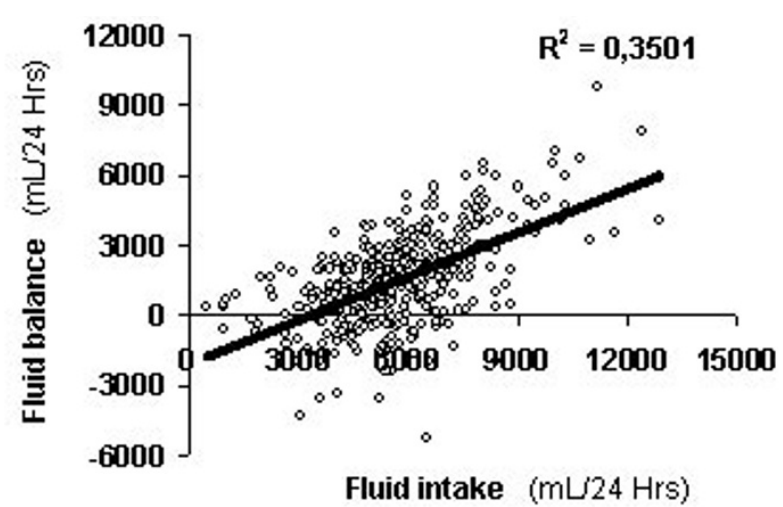

Relationship between fluid intake and fluid balance in nonsurvivors (average fluid intake, $5810 \pm 1800 \mathrm{ml}$; average fluid balance, $1580 \pm 1900 \mathrm{ml}$; average value of CRP, $125 \pm 62 \mathrm{mg} / \mathrm{l})$. 
Figure 2

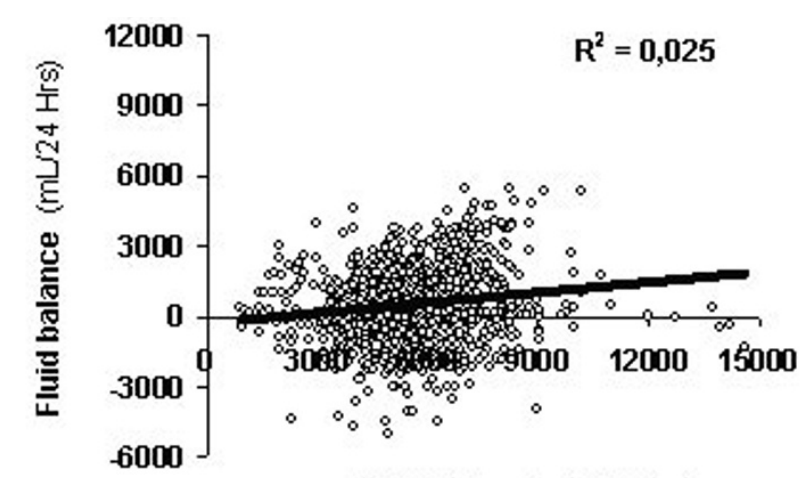

Fluid intake (mL/24 Hrs.

Relationship between fluid intake and fluid balance in survivors (average fluid intake, $5539 \pm 1160 \mathrm{ml}$, not significant compared to nonsurvivors; average fluid balance, $523 \pm 1480 \mathrm{ml}, P<0.05$ compared to nonsurvivors; average value of CRP, $92 \pm 60 \mathrm{mg} / \mathrm{l}, P<0.05$ compared to nonsurvivors).
Figure 3

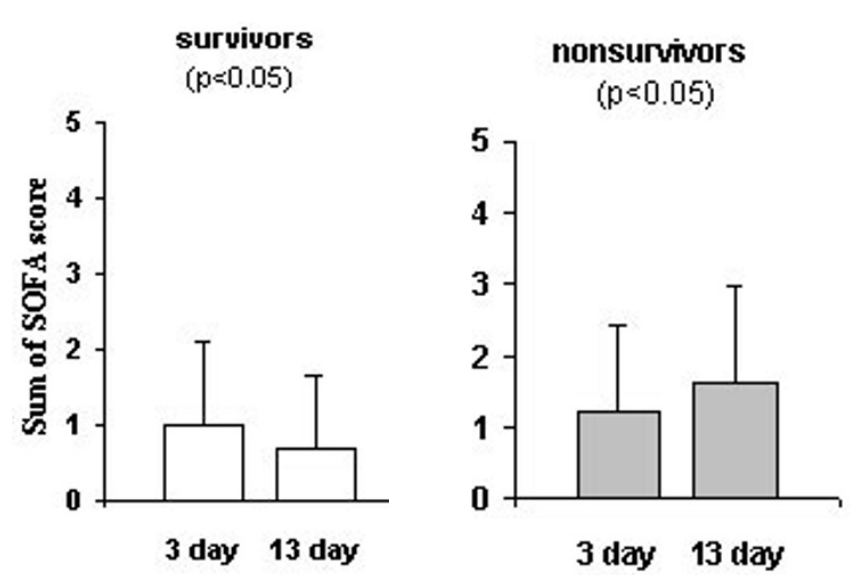

Organ dysfunction development.

\section{P20 Effects of volume replacement on plasma volume and albumin escape rate in a porcine model of fecal peritonitis}

G Marx*, B Vangerow ${ }^{*}$, M Cobas Meyer*, T Schuerholz*, R Sümpelmann*, L Wilkens ${ }^{\dagger}$, KF Gratz ${ }^{\ddagger}$, M Leuwer $^{*}$ and H Rueckoldt ${ }^{*}$ Departments of Anaesthesiology, ${ }^{\dagger}$ Pathology and ${ }^{\ddagger}$ Nuclear Medicine, Hannover Medical School, D-30625 Hannover, Germany

Objectives: We investigated effects of $4 \%$ and $8 \%$ gelatinbased solutions, hydroxyethyl starch (HES) and ringer's solution on albumin escape rate (AER) and maintenance of plasma volume (PV) in a porcine fecal peritonitis model.

Design: This was a prospective randomized, controlled animal laboratory study.

Measurement and main results: Fasted, anaesthetized, mechanically ventilated and multi catheterized pigs $(20.8 \pm 1.8 \mathrm{~kg})$ received $1 \mathrm{~g}$ of feces per $\mathrm{kg}$ of body weight into the abdominal cavity to induce sepsis and were observed over eight $h$. At induction, animals were randomized to resuscitation with gelatin $8 \%(n=5)$, gelatin $4 \%$ $(n=5), 6 \%$ HES 200/0.5 $(n=5)$, ringer's solution $(n=5)$ and compared to a nonseptic control group $(n=5)$ receiving $6 \%$ HES 200/0.5. The infusion rate was set to maintain a central venous pressure of $12 \mathrm{mmHg}$. Red blood cell volume was analyzed using chromium-51-tagged erythrocytes (RBC: ${ }^{51} \mathrm{Cr}$ ). PV was determined from standard formulae. AER was calculated using $99 \mathrm{~m}$-labeled technetium serum albumin. Systemic hemodynamics and oxygenation

Table

\begin{tabular}{|c|c|c|c|c|c|}
\hline & Gelatin 8\% & Gelatin 4\% & HES & Ringer & Control (HES) \\
\hline \multicolumn{6}{|l|}{ RBC: ${ }^{51} \mathrm{Cr}[\mathrm{ml} / \mathrm{kg}]$} \\
\hline Preshock & $20.1 \pm 1.9$ & $21.1 \pm 2.6$ & $22.1 \pm 2.5$ & $23.2 \pm 2.6$ & $24.7 \pm 1.6$ \\
\hline $8 \mathrm{~h}$ & $20.2 \pm 1.7$ & $19.8 \pm 2.8$ & $21.1 \pm 2.0$ & $18.9 \pm 1.4$ & $24.6 \pm 1.5^{*}$ \\
\hline \multicolumn{6}{|l|}{$\mathrm{PV}:{ }^{51} \mathrm{Cr}[\mathrm{ml} / \mathrm{kg}]$} \\
\hline Preshock & $60.0 \pm 5.1$ & $57.3 \pm 5.9$ & $59.0 \pm 8.1$ & $61.0 \pm 2.2$ & $65.5 \pm 7.7$ \\
\hline $8 \mathrm{~h}$ & $73.9 \pm 7.9^{*}$ & $54.8 \pm 9.5$ & $59.1 \pm 11.1$ & $32.9 \pm 2.9^{*}$ & $79.8 \pm 6.8^{*}$ \\
\hline Infusion volume $[\mathrm{ml} / \mathrm{kg}] 8 \mathrm{~h}$ & $105 \pm 15$ & $160 \pm 25$ & $146 \pm 43$ & $401 \pm 84^{*}$ & $108 \pm 31$ \\
\hline \multicolumn{6}{|l|}{$\mathrm{SvO}_{2}[\%]$} \\
\hline Preshock & $71 \pm 7$ & $73 \pm 4$ & $73 \pm 5$ & $75 \pm 5$ & $78 \pm 5$ \\
\hline $8 \mathrm{~h}$ & $37 \pm 20$ & $37 \pm 13$ & $55 \pm 19^{*}$ & $42 \pm 17$ & $74 \pm 5^{\star}$ \\
\hline \multicolumn{6}{|l|}{ AER [\%] } \\
\hline $4 \mathrm{~h}$ & $24 \pm 9$ & $24 \pm 3$ & $22 \pm 4$ & $21 \pm 2$ & $3 \pm 3$ \\
\hline $8 \mathrm{~h}$ & $11 \pm 8$ & $10 \pm 3$ & $8 \pm 3$ & $8 \pm 2$ & $-2 \pm 4^{*}$ \\
\hline
\end{tabular}

${ }^{\star} \mathrm{P}<0.05$, intergroup differences using univariate analysis of variance (UNIANOVA). 
were obtained before induction of sepsis, and 4 and $8 \mathrm{~h}$ afterwards.

Conclusion: PV was increased by gelatin $8 \%$ only. This suggests a better remaining volume in circulation during sepsis as there were no differences between infusion volume in all colloid groups. On the other hand, systemic oxygenation was significantly better in animals treated with HES than with gelatin $8 \%$ or $4 \%$. In this respect, it is interesting to note that histological investigation of lung and kidney revealed a bluish blubbery fluid in small blood vessels, glomerula and tubules of the animals treated with gelatin $8 \%$ or gelatin $4 \%$. \\ The formation of platelet microvesicles in septic pigs treated with different kinds of volume replacement}

T Schuerholz*, G Marx*, B Vangerow*, M Cobas Meyer*, M Ballmaier†, J Heine*, HJ Schuberth‡, H Rueckoldt* and R Sümpelmann* ${ }^{*}$ Department of Anesthesiology, ${ }^{\dagger}$ Pediatric Hematology and Oncology, Hannover Medical School, 30625 Hannover, Germany; ${ }^{\ddagger}$ Immunology Unit ${ }^{3}$, Hannover School of Veterinary Medicine, Hannover, Germany.

Introduction: Bacterial lipopolysaccharide (LPS) is believed to be one of the major pathogenic factors of Gram-negative sepsis. Sepsis may be accompanied with thrombocytopenia, platelet activation and elevated platelet-derived microvesicles. Higher formation of microvesicles has been associated with higher mortality in endotoxemic pigs.

Design: Prospective, randomized animal laboratory study.

Methods: Twenty anesthetized pigs divided in four groups received feces ( $1 \mathrm{~g} / \mathrm{kg}$ body-weight) installed in the abdomen to induce sepsis. Group 1 was treated with ringer's solution (RS), group 2 with $6 \%$ hydroxyethyl starch 200/0.5 (HES), group 3 with modified fluid gelatin $4 \%$ (MFG4) and group 4 with modified fluid gelatin 8\% (MFG8), each to maintain a central venous pressure of $12 \mathrm{mmHg}$. Before induction of sepsis (baseline) and after 2 (+2 h), 4 (+4 h), and 8 (+8 h) h, a citrated blood sample was drawn. Flow cytometry was used for determination of microvesicles. Platelets and microvesicles were identified with an anti-platelet-mAb (Donor: DH Sachs, Charlestown, USA) and a secondary antibody labeled with phycoery- thrin (goat-anti-mouse, Dako SA, Denmark). Microvesicles were determined as smallest $1-3 \%$ positive cells in forward scatter.

Results: Baseline values were considered as $100 \%$. In the RS-group sepsis was associated with an increase of microvesicles to $137 \pm 58 \%$ (Mean $\pm S D$ ) after $2 \mathrm{~h}$, leading to $179 \pm 45 \%(+4 \mathrm{~h})$ up to $210 \pm 121 \%(+8 \mathrm{~h})$. In group 2 (HES) there was an increase at $+2 \mathrm{~h}(109 \pm 25 \%)$ and a decrease at $+4 \mathrm{~h}(89 \pm 26 \%)$ and $+8 \mathrm{~h}(73 \pm 19 \%)$. In Group 3 and 4 a decrease of microvesicles after induction was revealed at $+2 \mathrm{~h}(51 \pm 25 \%$, MFG4 and $45 \pm 16 \%$, MFG8) +4 h (62 $\pm 10 \%$, MFG4, 79 $\pm 58 \%$, MFG8) and $+8 \mathrm{~h}(63 \pm 41 \%$, MFG4, $53 \pm 17 \%$ MFG8). Formation of microvesicles was significantly higher in the RS treated group compared to all colloid groups $(P<0.05)$.

Conclusion: In this porcine sepsis model, volume replacement with colloid solutions seemed to reduce the detectable number of platelet derived microvesicles, which could be seen after the use of ringers solution. This may be a sign of less activation with colloids.

P22 The changes in platelet function in SIRS, sepsis and MODS - a tight connection to the changes in the immune and hemostatic system

\section{U Leonhardt, M Koksch, G Röthig and L Engelmann}

University Leipzig, Department of Internal Medicine I, Philipp-Rosenthal-Strasse 27, 04103 Leipzig, Germany

Background: In previous studies platelet dysfunction was described as a part of disseminated intravascular coagulation (DIC). These findings are also important in septic patients. In this present study, the association of the platelet function with the systemic inflammation and the development in different parts of the septic process in patients of an internal intensive care unit were investigated.

Methods: Twenty-five patients fulfilling clinical, hemodynamic and blood chemistry results of a systemic inflammation were included. The investigations were done in a two-day period. The characterization of the immune-state with IL-6, TNF- $\alpha$ and Procalcitonin used standard methods. The platelet activation marker P-selectin (GMP140) was analyzed by flow cytometric detection ex vivo and after stimulation using $5 \mu \mathrm{M}$ ADP and $10 \mu \mathrm{M}$ TRAP-6. $\mathrm{DIC}$ was also characterized by standard laboratory results (platelet count, aPTT, AT III, fibrinogen, TAT, D-Dimer). The APACHE II-score evaluated the clinical situation.

Results: The activation status of platelets was significantly associated with the process of inflammation. Pre-activated platelets (ex vivo) were seen in all patients with systemic inflammation (PCT $P<0.03$ ). During the measurement over a two-day period (five results for each patient) the pre-activation and the reagibility of TRAP-6-stimulation were significantly correlated to plasma levels of IL-6 $(P<0.01)$ and TNF- $\alpha(P<0.04)$. Furthermore the detection of changes in platelet function started earlier then the measured results of common laboratory tests in DIC. 
Conclusion: Platelet function was tightly associated with the process of systemic inflammation. The dysfunction of the cellular part of coagulation could be an important marker of changes in the hemostatic system and the development of the disseminated intravascular coagulation.

P23 Newly developed thrombocytopenia in medical intensive care patients

\section{R Strauss, M Wehler, K Mehler, D Kreutzer, A Mueller, P Martus* and EG Hahn}

Departments of Medicine I and *Medical Informatics, Biometry and Epidemiology, University Erlangen-Nuremberg,

Krankenhausstrasse 12, D-91054 Erlangen, Germany

Introduction: Thrombocytopenia is a major concern in intensive care medicine. The incidence is greatly varying depending on the clinical setting.

Objective: To determine the incidence, severity, prognosis and therapeutic implications of thrombocytopenia in our 12-bed medical (noncoronary) ICU.

Methods: We evaluated, in a prospective observational study over 13 months (1.11.1997-30.11.1998), all patients who stayed longer than $48 \mathrm{~h}$ in the ICU. Thrombocytopenia was defined as a platelet count below $150000 / \mu l$.

Results: We studied 243 patients (63.3\% male) with a mean age of $57.9( \pm 16.34,18-96)$ years, a mean APACHE II score of $21.2( \pm 10.06,0-49)$ and a mean length of stay of 13.5 days. Thrombocytopenia was observed frequently: only 82 patients (33.7\%) were never thrombocytopenic. Ninety-nine (40.7\%) patients were thrombocytopenic on admission. From the 144 patients who had normal platelets on admission 62 (45.2\%) developed thrombocytopenia. In this group of patients, ICU mortality was significantly correlated with the degree of thrombocytopenia: $66.7 \%$ in patients with platelets $<20000 / \mu \mathrm{l}, \quad 55.6 \%$ in patients with platelets 20000-50000, 47.4\% in patients with platelets $50000-100000,9.7 \%$ in patients with platelets $100000-150000\left(P=0.003 \chi^{2}\right)$.

Conclusion: $45.2 \%$ of patients became thrombocytopenic during their ICU stay. The degree of newly developed thrombocytopenia was highly correlated with mortality, length of stay, initial APACHE II score and the consumption of blood products.

\begin{tabular}{|c|c|c|c|c|c|c|c|}
\hline \multicolumn{2}{|c|}{ Platelets during the ICU stay } & \multirow[b]{2}{*}{$\begin{array}{c}\text { Packed red blood cells } \\
\text { units }\end{array}$} & \multirow[b]{2}{*}{$\begin{array}{l}\text { FFPs } \\
\text { units }\end{array}$} & \multirow[b]{2}{*}{$\begin{array}{l}\text { SDAP (platelets) } \\
\text { units }\end{array}$} & \multirow[b]{2}{*}{$\begin{array}{l}\text { SOFA on } \\
\text { admission }\end{array}$} & \multirow[b]{2}{*}{$\begin{array}{l}\text { APACHE II } \\
\text { on admission }\end{array}$} & \multirow[b]{2}{*}{$\begin{array}{l}\text { Length of stay } \\
\text { days }\end{array}$} \\
\hline$/ \mu l$ & $\mathrm{~N}$ & & & & & & \\
\hline $100001-150000$ & 31 & $2.29 \pm 4.03$ & 0 & 0 & $4.81 \pm 2.70$ & $21.42 \pm 9.56$ & $17.06 \pm 18.88$ \\
\hline $50001-100000$ & 19 & $3.58 \pm 5.67$ & $2.84 \pm 5.40$ & $0.05 \pm 0.23$ & $6.63 \pm 2.93$ & $27.00 \pm 8.08$ & $13.79 \pm 11.02$ \\
\hline $20000-50000$ & 9 & $10.00 \pm 7.45$ & $4.22 \pm 7.03$ & $0.67 \pm 1.12$ & $7.67 \pm 2.87$ & $25.78 \pm 12.22$ & $23.22 \pm 27.92$ \\
\hline$<20000$ & 3 & $28.33 \pm 18.18$ & $17.67 \pm 4.73$ & $6.67 \pm 5.51$ & $8.67 \pm 3.21$ & $35.00 \pm 6.93$ & $55.67 \pm 64.63$ \\
\hline$P$ & & $<0.001$ & 0.027 & 0.003 & 0.075 & 0.002 & $<0.001$ \\
\hline
\end{tabular}

All data mean \pm standard deviation. ANOVA was used for testing.

P24 Effects of Antithrombin III on body cavity effusions, fluid balance, colloid osmotic pressure and hemodynamics in porcine septic shock

M Cobas Meyer, B Vangerow, J Ahrens, T Schuerholz, G Marx and H Rueckoldt

Department of Anaesthesiology, Hannover Medical School, D-30625 Hannover, Germany

Introduction: It has been suggested that Antithrombin III (AT III) might have an anti-inflammatory effect in addition to its well known anti-thrombotic properties. The aim of this study was to investigate the effects of AT III on abdominal and pleural effusions, fluid balance, colloid osmotic pressure (COP) and hemodynamics, in a porcine fecal peritonitis model.

Methods: This was a prospective randomized, controlled animal laboratory study. Fasted, anaesthetized, mechanicallyventilated and multi-catheterized pigs $(20.6 \pm 1.9 \mathrm{~kg})$ received $1 \mathrm{~g} / \mathrm{kg}$ body weight feces into the abdominal cavity to induce sepsis and were observed over $8 \mathrm{~h}$. Before induction, animals were randomized to treatment with 200 IE/ kg AT III (Kybernin ${ }^{\circledR}$ P, Centeon Pharma GmbH; Marburg, Germany) ( $n=4)$ or to controls ( $\mathrm{NaCl} 0.9 \%)(n=4)$.

Hemodynamic treatment scheme with ringer's solution was based on ensuring baseline intrathoracic blood volume (ITBV). Hemodynamics and COP were measured before sepsis induction and $4 \mathrm{~h}$ and $8 \mathrm{~h}$ afterwards. Cumulative fluid balance was calculated after 4 and $8 \mathrm{~h}$. Abdominal and pleural effusion volumes were obtained after sacrificing the animals.

Results: See Table. 


\begin{tabular}{|c|c|c|c|c|c|c|}
\hline \multirow[b]{2}{*}{ Group } & \multicolumn{2}{|c|}{ before } & \multicolumn{2}{|c|}{$+4 \mathrm{~h}$} & \multicolumn{2}{|c|}{$+8 \mathrm{~h}$} \\
\hline & controls & AT III & controls & AT III & controls & AT III \\
\hline Abdominal effusion [ml] & & & & & $1125 \pm 96^{*}$ & $588 \pm 103^{*}$ \\
\hline Pleural effusion [ml] & & & & & $175 \pm 87^{\star}$ & $60 \pm 32^{*}$ \\
\hline $\mathrm{COP}[\mathrm{mmHg}]$ & $10.1 \pm 3,3$ & $10.1 \pm 1.6$ & $5.2 \pm 0.5^{\star}$ & $7.8 \pm 1.8^{\star}$ & $3.3 \pm 0.9$ & $5,7 \pm 1.5$ \\
\hline Cumulative fluid balance [l] & & & $3.3 \pm 0.9^{*}$ & $1.4 \pm 0.2^{*}$ & $7.3 \pm 2.8$ & $4.1 \pm 1.5$ \\
\hline ITBV [ml] & $357 \pm 03$ & $383 \pm 57$ & $353 \pm 143$ & $356 \pm 89$ & $306 \pm 149$ & $291 \pm 61$ \\
\hline CVP [mmHg] & $10 \pm 3$ & $10 \pm 2$ & $10 \pm 4$ & $10 \pm 2$ & $15 \pm 2$ & $12 \pm 5$ \\
\hline PAWP [mmHg] & $12 \pm 3$ & $13 \pm 1$ & $12 \pm 5$ & $9 \pm 1$ & $11 \pm 2$ & $11 \pm 2$ \\
\hline $\mathrm{MAD}[\mathrm{mmHg}]$ & $118 \pm 6$ & $119 \pm 12$ & $110 \pm 14$ & $103 \pm 11$ & $60 \pm 8$ & $67 \pm 12$ \\
\hline
\end{tabular}

(*) $P<0.05$; difference between the groups (Student's t-test with Bonferroni Holm correction).

Conclusion: In the AT III treated group both abdominal and pleural effusion volumes were lower than in the controls. COP values $4 \mathrm{~h}$ after sepsis induction were higher and the cumulative fluid balance lower. This may indicate that AT III has a positive effect on capillary leakage in septic shock.

\section{P25 Antithrombin (AT) improves inflammation induced microcirculatory disturbance in rat mesentery}

B Leithäuser, S Lendemans, J Schumacher, H Tillmanns and FR Matthias

Medical Clinic I, Department of Cardiology-Angiology, Justus-Liebig-University, Klinikstrasse 36, 35385 Giessen, Germany

Background: Impairment of the microcirculation is one of the main causes for multiple organ failure (MOF) in critical care patients. Trials with AT substitution in patients with sepsis showed a positive effect on the development and course of MOF. Recent experimental studies indicate that there might be specific AT-effects independent of the coagulation cascade, which can modulate endothelialleukocyte interaction and vascular permeability.

Methods: The effect of AT substitution on LPS-induced microvascular leakage (ML) and leukocyte adhesion (LA) in the rat mesentery was investigated by means of intravital microscopy. Male CD rats were infused with $0.5 \mathrm{mg} / \mathrm{kg}$ LPS (E. coli) over $80 \mathrm{~min}$. Vascular leakage was detected with FITC-marked rat serum albumin by fluorescence microscopy and evaluated by grey-value analysis with image processing software. Light microscopy was used to evaluate leukocytes adherence to the vessel wall. Two treated groups received $500 \mathrm{U} / \mathrm{kg}$ AT either $20 \mathrm{~min}$ prior to or $20 \mathrm{~min}$ after the begin- ning of LPS-infusion. Animals not infused with LPS, either untreated or treated with placebo (albumin), served as controls. One pre-treated group additionally received heparin at a clinically used dosage. Furthermore, interleukin-2 (IL-2) was used to induce ML and treated with AT prior to infusion.

Results: LPS-infusion led to a significant increase of ML and LA compared to controls. Both effects were reduced to the level of controls by the substitution of AT. No significant differences were found between the pre-treated or the post-treated group. IL-2 induced ML was also reduced by treatment with AT.

Conclusion: Substitution of AT, even when given after LPS, ameliorates vascular leakage and leukocyte adhesion to the vessel wall. Together, these effects improve flow conditions in the microcirculation. Hence IL-2-induced leakage could also be modulated by AT and a direct effect by AT on leukocyte adhesion seems to be likely.

\section{P26 G-protein- and phosphodiesterase-dependent regulation of neutrophil migration by antithrombin III involving CXC-receptor 1}

S Dunzendorfer, A Rabensteiner, N Kaneider, J Römisch* and CJ Wiedermann

Department of Internal Medicine, University of Innsbruck, Innsbruck, Austria; *Centeon Pharma GmbH, Research, Marburg, Germany

Background: Antithrombin III (ATIII) is a serpin with a newly uncovered regulatory role in neutrophil (PMN) chemotaxis. ATIII and platelet factor-4 (PF4) differentially inhibited migration of PMN toward the CXC-chemokines, interleukin-8 (IL-8) and GRO- $\alpha$, and the classical attractants, fMet-Leu-Phe (fMLP) and C5a.

Aim: To determine signalling events and involvement of CXC-receptors (CXCR) 1 and 2 in ATIII-induced inhibition of directed PMN migration, we studied the in vitro effects of ATIII in the presence of specific CXCR1 and CXCR2 antibodies (mAb), pertussis toxin or various blockers of signalling enzymes of PMN.

Methods: As in the absence of other attractants, highly purified ATIII has been shown to itself induce PMN migration, effects were determined in modified Boyden chemotaxis chambers by the micropore-filter leading front assay 
in a 48-well system, after pretreatment of PMN with antibodies, pertussis toxin or enzymes blockers.

Results: Preincubation of PMN with effective concentrations of pertussis toxin, staurosporine or 3-isobutyl-1methylxantine completely blocked ATIII-induced migration whereas treatment with bisindolylmaleimide (GFX), a selective protein kinase $\mathrm{C}$ blocker, wortmannin or tyrphostin-23, had no effect. In assays of IL-8-induced migration, PMN responses to the enzyme blockers were comparable, whereas PF4-induced responses differed as they were also sensitive to GFX. Migration of PMN toward
ATIII was significantly reduced by pre-treatment of cells with CXCR1 but not CXCR2 mAb, whereas migration toward IL-8 was antagonised by both CXCR1 and CXCR2 mAb. The mAbs had no effect on fMPL-induced $\mathrm{PMN}$ migration. $\mathrm{A}$ mAb to IL-1R, which was used as a control, was inactive as well.

Conclusion: Effects of ATIII on PMN migration appear to involve specific signalling pathways including CXCR1, Gproteins and phosphodiesterase and staurosporine-sensitive enzymes other than protein kinase $C$ (i.e. protein kinase A or phospholipase D).

\section{Inhibition of neutrophil migration by the serpin antithrombin III}

\section{S Dunzendorfer, A Rabensteiner, C Reinisch, J Römisch* and CJ Wiedermann}

Department of Internal Medicine, University of Innsbruck, Innsbruck, Austria; *Centeon Pharma GmbH, Research, Marburg, Germany

Background: Beyond the well-known regulatory role of antithrombin III (ATIII) in haemostasis, anti-inflammatory effects have been described such as the induction of prostacyclin release by endothelial cells or the reduction of tissue accumulation of neutrophils (PMN) in animal models of ischemia-reperfusion and sepsis.

Aim: To determine a role of ATIII beyond its ability to reduce leukocyte-mediated events induced by thrombin, we studied direct effects on the in vitro migration and respiratory burst activity of PMN by the ATIII concentrate Kybernin ${ }^{\circledR} \mathrm{P}$, the monoclonal antibody-purified ATIII thereof and its $\alpha$ and $\beta$ isoforms.

Methods: PMN of healthy donors were isolated by lymphoprep ${ }^{\circledR}$ followed by hypotonic lysis of contaminating erythrocytes. Chemotactic activity was determined in modified Boyden chambers by the micropore-filter leading front assay in a 48-well system. Respiratory burst activity of PMN was measured fluorimetrically.
Results: Pre-incubation in vitro of human PMN with Kybernin ${ }^{\circledR} \mathrm{P}$, immune-adsorbed ATIII or both isoforms significantly inhibited the migration toward fMet-Leu-Phe (fMLP), interleukin-8 (IL-8), and ATIII itself, in a concentration-dependent manner following a bell-shaped curve. Maximum ATIII effects were seen at a concentration of $1 \mathrm{U} / \mathrm{ml}$, which is the level of ATIII found in normal human plasma. Purified ATIII did not deactivate PMN chemotaxis toward GRO- $\alpha$, whereas fMLP, IL-8, platelet factor-4 (PF4) and GRO- $\alpha$ itself did. Checkerboard analyses indicate that the inhibition of migration was not due to a general impairment of the chemotactic activity of the cells but most probably involved receptor regulatory processes. Deactivation of chemotaxis toward IL- 8 but not GRO- $\alpha$ suggests differential interaction at the CXC receptors 1 and 2. At comparable concentrations purified ATIII and Kybernin ${ }^{\circledR} \mathrm{P}$ did not affect resting or stimulated respiratory burst activity of PMN.

Conclusion: Our results suggest that ATIII may be a physiologic regulator of the acute inflammatory response by protecting cells from premature activation.

\section{P28 Lipopolysaccharide induced procoagulant activity and cytokine production: influence of antithrombin}

E Gray, P Souter, J Römisch* and S Poole

NIBSC, Blanche Lane, South Mimms, Potters Bar, Herts EN6 3QG, UK; *Centeon Pharma GmbH, 35002 Marburg, Germany

Sepsis-induced disseminated intravascular coagulation is usually caused by endotoxin evoked production of cellular procoagulant tissue factor (TF) and pro-inflammatory cytokines, which further perpetuate the generation of TF. Large doses of antithrombin (AT) reduce mortality and morbidity in septic patients and there is increasing evidence to suggest that AT has anti-inflammatory properties in addition to its anticoagulant properties. In the present study, we investigated the effect of AT in three in vitro cellular systems: citrated whole blood, human umbilical vein endothelial cells (HUVECs) and mononuclear cells (MNCs). The cells were stimulated with lipopolysaccharide
(LPS) for 4-6 $\mathrm{h}$ in the presence and absence of AT. Procoagulant tissue factor (TF) activity was estimated by a TFdependent clotting or chromogenic assay and interleukin- 6 (IL-6) was measured by ELISA. In all three systems, 5-40 $\mathrm{IU} / \mathrm{ml}$ AT was found to inhibit TF and IL-6 production in a dose-dependent manner. This inhibitory effect was not attributable to excipients or co-purified components of AT. Experiments with chemically modified AT and a low heparin binding fraction of AT indicated that binding to heparin and/or cell surface glycosaminoglycans is important for the inhibitory activity. Up to $40 \mu \mathrm{M}$ of a specific thrombin inhibitor, r-hirudin, did not inhibit the production of TF or IL- 
6 in either of three cellular systems, suggesting that inhibition of thrombin might not be the main mechanism by which AT prevents the production of TF and IL-6. The results of this study have shown that, apart from the inhibi- tion of thrombin and other activated coagulation factors, AT may also downregulate the cellular expression of proinflammatory responses and therefore may have an added value in the treatment of sepsis-induced DIC.

\section{P29 Effects of antithrombin III (ATIII) treatment (high dose) in severe pre-eclampsia and HELLP syndrome with alterations of coagulation inhibitors and inflammatory markers: a preliminary report}

A Giarratano, G Cuccio* and S Mangione ${ }^{\dagger}$

Istituto Materno Infantile, ${ }^{+}$Cattedra di Anestesia e Rianimazione, *Cattedra di Terapia Intensiva, Università degli Studi di Palermo, via C Rampolla 1, 90142-Palermo, Italy

Objective: Several investigations and our recent experience indicate that the intravascular inflammatory response and the clotting alterations registered during severe preeclampsia and HELLP syndrome are not an epiphenomenon but the cause of the clinical syndrome. The aims of this study were to investigate the effects of the ATIII substitutive treatment on cytokine plasma concentrations, assumed as a marker of endothelial damage and of the associated systemic inflammatory response. A secondary objective was to correlate the ATIII treatment with the evolution of both single or multi-organ dysfunction syndrome (MODS).

Materials and methods: The study involved four patients with severe pre-eclampsia and three HELLP syndrome patients. Diagnostic criteria for severe pre-eclampsia were those published by the American College of Obstetricians and Gynecologists (diastolic blood pressure $>110 \mathrm{mmHg}$ and proteinuria $\geq 0.5 \mathrm{~g} / \mathrm{l}$ ). Diagnostic criteria for HELLP syndrome required, in association with hypertension and proteinuria, thrombocytopenia $(<150000$ cells $/ \mu \mathrm{l}$ ), evidence of hepatic dysfunction (aspartate aminotransferase [AST] and alanine aminotranserase [ALT] levels $>40 \mathrm{IU} / \mathrm{L}$ with lactate dehydrogenase [LDH] level of $>600 \mathrm{IU} / \mathrm{I}$ ) and evidence of hemolysis (increased $\mathrm{LDH}$ and anemia). Plasma levels of tumor necrosis factor (TNF $\alpha$ and interleukins [IL1 $\beta$ and IL6]) were measured by enzyme-linked immunoadsorbent assay (ELISA). Plasma concentrations of Antithrombin III (ATIII) and Protein C (P. C) were measured by a chromogenic assay. ATIII was administered after the first sampling (admission) by a loading dose of $3.000 \mathrm{IU}$ in bolus infusion and a maintenance dose of $1500 \mathrm{U} / 12 \mathrm{~h}$ over 4 days. Results were compared using the Mann-Whitney test. Maternal parameters and clinical data were compared using unpaired Student t-test.

Results and discussion: Clinical data are listed in Table 1 and results are shown in Table 2 . These results demonstrate significant changes in fibronectin and ATIII concentrations between levels at admission compared with levels after ATIII treatment. It is very interesting to register a decrease in blood levels of cytokines and a reduced proinflammatory activity. The pro-inflammatory activity was increased in women with severe pre-eclampsia and HELLP syndrome patients and this result could also be explained by specific effects which are independent from the coagulation cascade (anti-inflammatory actions).

Conclusions: The preliminary results of this study confirm our hypothesis as well as the utility of marker monitoring and substitutive treatment. We believe that it is necessary to develop a large study (as a phase III trial) to confirm our hypothesis and to achieve other significant results.

\section{References:}

1. Redman CWG. et al:: Am J Obstet Gynecol 1999, 180:499-506.

2. Okajima $K$ et al.: Thromb Res 1998, 24:27-32.

3. Souter et al:: Int Care Med 1999,19

Table 1

\begin{tabular}{lcc}
\hline Clinical data & & \\
\hline & $\begin{array}{c}\text { Severe } \\
\text { Preeclampsia } \\
(\mathrm{n}=4)\end{array}$ & $\begin{array}{c}\text { HELLP } \\
\text { Syndrome } \\
(\mathrm{n}=3)\end{array}$ \\
\hline Age(y) & $28.2(5.9)$ & $29.1(4.8)$ \\
Diastolic pressure & $98(9)$ & $101(6)$ \\
AST/ALT (IU) (Admission) & $29 / 22$ & $201 / 158$ \\
AST/ALT (IU) (Peak) & $49 / 31$ & $451 / 254$ \\
Proteinuria (dipstick) & $1+/ 2+$ & $3+/ 4+$ \\
Gestational age at admission (w) & $33.1(0.2)$ & $32.8(0.2)$ \\
Gestational age at delivery (w) & $33.2(0.2)$ & $33.1(0.2)$ \\
\hline
\end{tabular}

Data were presented as mean $( \pm s d)$.

Table 2

\begin{tabular}{lcc} 
Markers and cytokines levels \\
\hline & $\begin{array}{c}\text { Levels at Admission } \\
(\mathrm{n}=7)\end{array}$ & $\begin{array}{c}\text { Levels after } \\
\text { ATIII treatment }(\mathrm{n}=7)\end{array}$ \\
\hline $\mathrm{IL} 1 \beta(\mathrm{pg} / \mathrm{mL})$ & $7.11(3.33-7.52)$ & $3.43^{\star}(2.11-6.06)$ \\
$\mathrm{IL} 6(\mathrm{pg} / \mathrm{mL})$ & $8.75(3.67-8.08)$ & $4.23^{\star}(2.55-7.02)$ \\
TNF $\alpha(\mathrm{pg} / \mathrm{mL})$ & $21.2(18.8-33.6)$ & $18.1^{\star}(11.3-19.9)$ \\
Fibronectin $(\mu \mathrm{g} / \mathrm{ml})$ & $592(497-701)$ & $444^{\star}(417-490)$ \\
AT III $(\%)$ & $52(38-62)$ & $91^{\star *}(78-96)$
\end{tabular}

$\mathrm{IL}=$ Interleukin; TNF $\alpha=$ tumor necrosis factor $\alpha$; AT III=antithrombin III; $\mathrm{PC}=$ Protein $\mathrm{C}$. Data are presented as median concentration (range). ${ }^{*} P<0.01$ compared with levels at admission (Mann Whitney $U$ test). ${ }^{* \star} P<0.001$ compared with levels at admission (Mann Whitney $U$ test). 


\section{P30 Decreased Protein C, Protein S and Antithrombin III Levels are predictive of poor outcome in} Gram-negative sepsis caused by Burkholderia pseudomallei

\section{SP Larosa*, SM Opal†, B Utterback*, B Yan*, J Helterbrand*, AJ Simpson ${ }^{\ddagger}$, N White ${ }^{\ddagger}$ and CJ Fisher*}

${ }^{*}$ Lilly Research Laboratories, Indianapolis, IN, USA; ${ }^{+}$Brown University, Providence, RI, USA; ${ }^{\ddagger}$ Mahidol University, Bangkok, Thailand

The acute septicemic form of Burkholderia pseudomallei infection or Melioidosis is associated with substantial release of endotoxin, TNF- $\alpha$ and IL-1. This inflammatory response leads to endothelial injury, activation of the extrinsic coagulation cascade, depletion of naturally occurring anticoagulants, microvascular thrombosis, organ failure and death. Plasma samples drawn at baseline and at time points during the illness from 30 patients with Melioidosis were assayed for D-dimer levels, Protein C and Protein $S$ antigen levels and Antithrombin III functional activities. Results of samples drawn during the illness were averaged for each patient. Baseline and continued deficiencies of Protein C, Protein S and Antithrombin III were predictive of poor outcome in a statistically significant fashion by logistic regression.

Endothelial injury as a result of inflammatory response to Burkholderia pseudomallei infection leads to coagulopathy and depletion of the natural anticoagulants Protein C, Protein S and Antithrombin III. Early and continued deficiency of these factors is predictive of poor outcome. Replacement therapy of depleted factors to achieve normal levels may be a worthwhile strategy for patients with Gram-negative sepsis.

\begin{tabular}{|c|c|c|c|c|c|c|c|}
\hline \multirow[b]{2}{*}{ * \pm std Assay } & \multirow[b]{2}{*}{ Outcome } & \multicolumn{3}{|c|}{ baseline } & \multicolumn{3}{|c|}{ averaged } \\
\hline & & $\#$ & mean* & $P$-value & \# & mean* & $P$-value \\
\hline Protein C (\%) ag & died & 12 & $43.4 \pm 18.0$ & 0.01 & 12 & $39.7 \pm 13.8$ & 0.004 \\
\hline nl. $(74-140)$ & survived & 18 & $74.6 \pm 27.8$ & & 18 & $80.8 \pm 26.9$ & \\
\hline Protein S (\%) ag & died & 12 & $65.8 \pm 17.9$ & 0.01 & 12 & $61.8 \pm 16.1$ & 0.006 \\
\hline nl. (55-116) & survived & 18 & $89.1 \pm 18.6$ & & 18 & $87.2 \pm 17.4$ & \\
\hline Anti-thrombin III (\%) & died & 12 & $71.0 \pm 20.0$ & 0.02 & 12 & $63.5 \pm 15.4$ & 0.005 \\
\hline nl. (84-124) & survived & 16 & $95.1 \pm 21.1$ & & 17 & $94.9 \pm 18.1$ & \\
\hline
\end{tabular}

P31 Hypercoagulability indicated by elevated blood TFPI (tissue factor pathway inhibitor) levels is closely related to severity of septic patients with glucose intolerance

M Hoshino, Y Haraguchi*, M Sakai, K Hayashi, N Horita, N Miyayama, H Saegusa and H Ohsawa

Department of Intensive and Critical Care Medicine, Tokyo Police Hospital, Fujimi 2-10-41, Chiyoda-ku 102-8161, Tokyo, Japan; *Tokyo Disaster Medical Center, Tokyo, Japan

Background and purpose: Hypercoagulability and endothelial cell activation and/or injury are mutually related and often found in acutely ill septic patients, and recently they have been reported to be related to multiple organ dysfunction syndromes (MODS).It is not clear, however, which parameters indicating coagulopathy are most closely related to MODS. In this report, we analyze correlations among the severity of the illness including MODS and parameters related to coagulopathy including TFPI in acutely ill septic patients, in order not only to ascertain their close relationships but also to find sensitive and predictive markers of the severity of septic patients.

Materials and methods: Five acutely ill septic patients with glucose intolerance were analyzed. Their blood glucose levels were strictly controlled by means of a bedside-type artificial pancreas (AP), STG-22, manufactured by NIKKISOH corporation in Japan. We selected septic patients in whom blood glucose levels were strictly controlled with STG-22, because some parameters related to coagulopathy, including Plasminogen Activator Inhibitor-1 (PAl-1), are influenced by blood glucose levels.

Analyzed items were: (1) regarding the severity of the illness, MOF score as the indicator of the degree of MODS (calculated from the MOF criteria of Japanese Association for Critical Care Medicine), blood thrombomodulin (TM) levels as the indicator of endothelial cell injury; (2) regarding coagulopathy, disseminated intravascular coagulation (DIC) score (calculated from the DIC criteria of the Ministry and Welfare of Japan), platelet count (PLT), fibrinogen, FDP, prothrombin time, TAT, D-dimer, PIC, plasminogen (PLG), Antithrombin (AT), Protein C activity, protein $S$ activity, total PAl-1, tissue plasminogen activator (tPA)-PAl-1 complex, and total TFPI; (3) regarding glucose tolerance, daily mean blood glucose level 
(BSm), $\mathrm{M}$ value (measured by the euglycemic hyperinsulinemic glucose clamp method with AP. The clamped blood glucose level was $80 \mathrm{mg} / \mathrm{dl}$, with the insulin infusion rate of $1.12 \mathrm{mU} / \mathrm{kg} . \mathrm{min}$ normal range $5-10 \mathrm{mg} / \mathrm{kg} . \mathrm{min})$.

Results: (1) Mean of $M$ value and BSm measured within a few days of admission (and during ICU stay) were $4.7 \pm 2.7$ $\mathrm{mg} / \mathrm{kg} \cdot \min \quad(n=4), \quad 176 \pm 10.7 \mathrm{mg} / \mathrm{dl} \quad(n=5) \quad[5.7 \pm 3.1$ $\mathrm{mg} / \mathrm{kg} \cdot \min (\mathrm{n}=12), 183.4 \pm 19.4 \mathrm{mg} / \mathrm{dl}(\mathrm{n}=65)]$, respectively. (2) There was strong positive relationship between MOF score and TM (correlation coefficient $r=0.95, n=32$, $P<0.005)$. (3) MOF score was correlated with TFPI $(r=0.91, n=30)$, PLT $(0.85, n=78)$, DIC score $(0.84$, $n=78)$, tPA-PAI $(0.76, n=33)$, AT $(0.66, n=32)$, PLG $(0.65, \mathrm{n}=33)$, and PIC (0.53, $\mathrm{n}=33),(P<0.005) .(4) \mathrm{TM}$ was correlated with TFPI $(0.89, n=29)$, tPA-PAI $(0.79$, $n=32)$, PLT (0.77, $n=32)$, DIC score $(0.75, n=32)$, PLG
(0.63, n=32), PIC (0.58, n=32), ( $P<0.005)$. (5) DIC score was correlated with PLT $(0.73, n=78)$, TFPI $(0.72, n=30)$, tPA-PAl (0.69, n=33), AT (0.61, $n=32),(P<0.005)$. (6) TFPI was correlated with tPA-PAI $(0.76, n=30)$, PLT $(0.70, \mathrm{n}=30), \mathrm{PIC}(0.50, \mathrm{n}=30),(P<0.005)$.

Interpretation and conclusions: The severity of disease in acutely ill septic patients, indicated by the progression of MODS and endothelial cell injury, was closely related to coagulopathy characterized by hypercoagulability with decreased fibrinolysis. Treatment for hypercoagulability seemed to be justified in severely septic patients. Although the mechanism was unclear, TFPI, which seemed to be a parameter of hypercoagulability and endothelial cell activation, was thought to be a sensitive and possibly predictive marker of the disease severity in septic patients.

P32 Endotoxin-induced capillary perfusion failure and leukocyte adhesion is not prevented by the thrombin inhibitor hirudin: an intravital microscopic study

JN Hoffmann*, B Vollmar', D Inthorn*, FW Schildberg* and MD Menger ${ }^{\dagger}$

${ }^{*}$ Department of Surgery Klinikum Großhadern, Ludwig-Maximilians-University Munich, 81377 München, Germany; ${ }^{\dagger}$ Department of Clinical and Experimental Surgery, University of Homburg/Saar, 66421 Homburg, Germany

Besides its central role in coagulatory pathways, thrombin is thought to be a key mediator of macrophage and granulocyte activation. During recent years the concept of coagulatory inhibition by the specific thrombin inhibitor hirudin has been established to treat septic disorders. Since basic mechanisms of sepsis include leukocyte-endothelial cell interaction as well as deterioration of capillary perfusion, we hypothesised that hirudin would modulate leukocyte activation and microvascular injury. In a skinfold preparation of Syrian hamsters, severe endotoxemia was induced by intravenous administration of endotoxin (LPS, E. coli, $2 \mathrm{mg} / \mathrm{kg})$. Hirudin $(0.25 \mathrm{mg} / \mathrm{kg})$ was substituted intravenously during $4 \mathrm{~h}$ after the induction of endotoxemia $[n=7$, hirudin group (hirudin)]. In control animals $[n=6$, Control group (control)], LPS was given without hirudin substitution. By intravital fluorescence microscopy leukocyte-endothelial cell interaction and functional capillary density (FCD, a measure of capillary perfusion) were analysed during a $24 \mathrm{~h}$ period after the LPS injection. Hirudin effectively normalised thromboplastin time and antithrombin activity when compared to controls $(P<0.05$, ANOVA). However, hirudin did not attenuate LPS-induced arteriolar and venular leukocyte adherence during $24 \mathrm{~h}$ $(P>0.05$, MANOVA). In parallel, hirudin caused significant deterioration in FCD over time when compared to controls (hirudin: baseline $=171 \pm 19$ vs $16 \pm 9$ at $24 \mathrm{~h}$; control: baseline $=150 \pm 20$ vs $62 \pm 18$ at $24 \mathrm{~h} ; P<0.05$ MANOVA). The decrease of FCD in hirudin animals was associated with a significant increase of wet-to-dry weight ratios in different organs (lung, kidney, muscle, and small intestine; $P<0.05$ vs control, ANOVA). Thus, our experiment does not indicate a protective effect of hirudin on microcirculation during endotoxemia, despite an improvement of coagulatory parameters. This result may at least in part explain the lack of efficacy of hirudin efficacy on lethality during endotoxemia and sepsis.

\section{P33 Heparin vs recombinant hirudin for anticoagulation in continuous renal replacement therapy}

\section{O Vargas Hein ${ }^{\dagger}$, C v Heymann+, J Nissen ${ }^{\dagger}$, M Lips ${ }^{\dagger}$, M Welte*, WJ Kox ${ }^{\dagger}$ and C Spies ${ }^{\dagger}$}

Departments of Anesthesiology and Intensive Care, *University Hospital Charite, Campus Mitte, Humboldt University Berlin, Schumannstr. 20/21, 10117 Berlin, Germany; ${ }^{\dagger}$ Benjamin Franklin Hospital, Free University Berlin, Hindenburgdamm 30,12200 Berlin, Germany

Introduction: Heparin as standard for anticoagulation in continuous renal replacement therapy (CRRT) has a bleeding incidence of $20 \%$ and is contraindicated in patients with heparin induced thrombocytopenia II [1]. Recently recombinant hirudin (rhirudin) has been reported to be superior as an anticoagulant to heparin for intermittent hemodialysis [2]. The aim of the study was to compare heparin and rhirudin as anticoagulants in CRRT regarding hemofiltration efficacy and possible bleeding complications.

Methods: After ethical committee approval and written informed consent from the relatives 16 critically ill patients with an indication for CRRT were randomly allocated to 2 
groups: heparin (9 patients) initially $250 \mathrm{IU} / \mathrm{h}$; target activated clotting time (ACT) 180-210 s, $125 \mathrm{IU} / \mathrm{h}$ stepwise heparin dose change; hirudin (8 patients) initially 10 $\mu \mathrm{g} / \mathrm{kg} / \mathrm{h}$; target ecarin clotting time (ECT) $80-100 \mathrm{~s}, 2$ $\mu \mathrm{g} / \mathrm{kg}$ stepwise dose change. Every four $\mathrm{h}$, thrombin time $(\mathrm{PT})$, partial thromboplastin time (PTT), hemoglobin $(\mathrm{Hb})$ and thrombocytes were determined. A bleeding complication was defined as an $\mathrm{Hb}$ decrease $>2 \mathrm{~g} / \mathrm{dl}$. The observation time was $96 \mathrm{~h}$. The ocurrence of filter clottings were recorded.

Statistical analysis: Mann-Whitney-U-Test, $P \leq 0.05$.

Results: Basic patient characteristics did not differ between groups.

Three bleeding complications were observed in three patients in the rhirudin group, but none were seen in the heparin group. The bleedings were observed $60 \mathrm{~h}$ after study initiation. Platelets were 76,14 and $62 / \mathrm{nl}$ at bleeding time. The ECT was 83,65 and 85 s respectively. PTT was higher than $60 \mathrm{~s}$ and PT was lower than $60 \mathrm{~s}$ at this point in all three patients. rhirudin application was inter-
Filter clotting and run time; median (range)

\begin{tabular}{lccc}
\hline & Heparin & RHirudin & $P$ \\
\hline Patients with filter clotting $(\mathrm{n})$ & $6 / 9$ & $3 / 8$ & 0.24 \\
Filter clottings/filter changes $(\mathrm{n})$ & $18 / 28$ & $8 / 22$ & 0.05 \\
Filter run time (h) & $12(4-36)$ & $20(4-56)$ & 0.18 \\
\hline
\end{tabular}

rupted 4 and $8 \mathrm{~h}$ before bleeding time point in two patients, the third patient received $5 \mu \mathrm{g} / \mathrm{kg} / \mathrm{h}$ rhirudin.

Conclusion: rhirudin prevented filter clotting more effectively than heparin. Low platelet count together with pathological PT and PTT, despite aimed or low ECT in patients with bleeding complications, indicated that improved clotting monitoring and dose adjustment must be implemented before rhirudin can be used safely.

\section{References:}

1. Vanholder R et al:: Kidney International 1994, 45:1754-1759.

2. vWyk V et al: Kidney International 1995, 48:1338-1343.

\section{P34 Using fraxiparin in patients with gestosis}

\section{Elioutine and DV Marshalov}

Saratov State Medical University, B. Kazachia 112, Saratov, 410026, Russia

Introduction: The problem of treating the coagulate disorders in cases of gestosis remains of topical interest in obstetrics; heparin is dangerous because of the possible development of thrombocytopenia, heparin-induced thrombosis, osteoporosis, and increased consumption of antithrombin III.

Methods: The investigation was carried out in 2 groups of pregnant women with gestosis. To the first group (consisting of 34 patients aged 18-32), the injections of fraxiparin (2850 ME) were given once a day. To the second group (consisting of 32 patients aged 19-30), the injections of heparin (5000) were given 4 times a day. The effectiveness of the drug was checked clinically and according to the investigations of coagulate and thrombocyte gemosta- sis the day before the operation and on the $1 \mathrm{st}, 4$ th and 7 th days after the operation.

Results: In the group of patients with gestosis who received heparin, reduction of the number of thrombocytes to $21.4 \%(P<0.01)$ and growth of their aggregation activity to $18.3 \%(P<0.05)$ were observed on the 7 th day of the postoperative period in comparison with the period prior to operation. In the group of pregnant women who received fraxiparin, a change in the number of thrombocytes and an increase in their aggregation activity were not observed.

Conclusion: The use of fraxiparin is more effective than treating with heparin and, as a result, it can be used for prophylaxis and treatment of thrombosis in pregnant women.

\section{P35 The effect of using a heparin-free flush system for central venous and pulmonary artery catheters} on a general medical and surgical intensive care unit

\section{Bradley and P Munro}

ITU, St George's Hospital, Blackshaw Road, London, SW17 OQT, UK

Introduction: Heparin flush systems prevent thrombus formation on invasive monitoring devices. Unfractionated heparin increases the risk of thrombocytopenia, and drug interactions in lines. Low dose heparin, given to most patients on the unit to prevent deep vein thrombosis (DVT), decreases thrombus formation on central venous catheters
(CVC). Pulmonary artery catheters (PAC) can be maintained with non-heparinised solutions but evidence for arterial lines is conflicting. Due to the high incidence of thrombocytopenia in our patients and the routine use of low molecular weight heparin to prevent DVT, we decided to investigate the use of a heparin-free flush system. 
Method: The study took place over two 4 week periods: one of heparin-free flush and one using a heparin flush. All patients on the unit were recruited into the study. Line care was according to unit practice. Duration of catheter patency and complications were recorded. Results were analysed using Chi squared and Fisher exact 2 tailed tests.

Results: The numbers of catheters in each group were:

\begin{tabular}{lcccc} 
& Arterial Catheter & CVC & PAC & Totals \\
\hline With Heparin & 65 & 48 & 14 & 127 \\
Heparin Free & 70 & 62 & 14 & 146 \\
Totals & 135 & 110 & 28 & 273
\end{tabular}

The numbers of thrombus associated problems were:

\begin{tabular}{lcccc} 
& Arterial Catheter & CVC & PAC & Totals \\
\hline With Heparin & 8 & 0 & 2 & 10 \\
Heparin Free & 7 & 1 & 1 & 9 \\
Totals & 15 & 1 & 3 & 19
\end{tabular}

There was no statistical difference in the thrombus-associated complication rates between the two groups.

Discussion: This study demonstrated that the removal of heparin from the flush systems of invasive monitoring lines does not lead to an increase in thrombus-associated complications, prevents the exposure to the risks of unfractionated heparin, which include thrombocytopenia and drug interactions, thereby improving patient care.

P36 Is the ecarin clotting time (ECT) a valid monitoring parameter for r-hirudin-based anticoagulation in continuous renal replacement therapy?

\author{
C v Heymann*, O Vargas Hein*, S Ziemer, P Sinha†, T Schröder*, J Nissen*, M Lips* and C Spies* \\ ${ }^{*}$ Department of Anaesthesiology and Intensive Care, ${ }^{\dagger}$ Institute of Laboratory Medicine and Pathological Biochemistry, University \\ Hospital Charité, Charité Campus Mitte, Schumannstrasse 20-21, D-10117 Berlin, Germany
}

Introduction: For continuous renal replacement therapy, systemic anticoagulation is required. Besides heparin, the current anticoagulant of choice, the direct acting thrombin-inhibitor r-hirudin was reported to be of advantage in chronic intermittent haemodialysis due to reduced filter clotting. The ecarin clotting time (ECT) was suggested to be a valid monitoring parameter with a linear correlation to $r$-hirudin concentrations in plasma [1]. To our knowledge, no results of hirudin-based anticoagulation during continuous renal replacement therapy in intensive care have been published yet. The objective of our study was to evaluate whether the ECT correlated to r-hirudin concentrations in plasma in this setting.

Methods: Seven patients on continuous veno-venous haemofiltration, due to acute renal failure, were included in this prospective study. Anticoagulation was titrated to achieve ECT-values between 80-100 s and parameters (ECT, PT, aPTT, TT, Fibrinogen, AT III, platelet count, ACT) were monitored by four-hourly determinations. R-hirudin concentrations in plasma were measured 24 and $48 \mathrm{~h}$ after starting therapy. Statistical analysis was performed by Spearman correlation.

Results: 5 of 14 measurements of hirudin-concentrations in plasma were found to be below the cutoff point of the $r$ hirudin test $(<0.1 \mu \mathrm{g} / \mathrm{ml})$. Six measurements were between 0.1 and $0.2 \mu \mathrm{g} / \mathrm{ml}$ and three results $>0.3 \mu \mathrm{g} / \mathrm{ml}$. For all measurements of our study we found a weak but statistically significant correlation between ECT and $r$ hirudin levels $(r=0.52 ; P=0.048)$. Within the narrow range
Figure

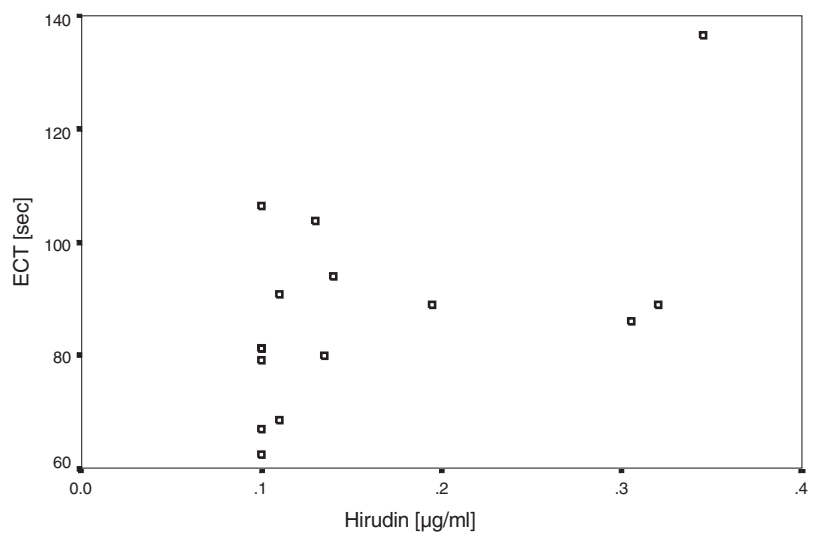

of r-hirudin levels between 0.1 and $0.2 \mu \mathrm{g} / \mathrm{ml}$, applying to 11 of 14 test results, a broad range of ECT values between 60 and 105 s. were found.

Conclusion: During CRRT very low plasma levels of $r$ hirudin ranging from $<0.1-0.35 \mu \mathrm{g} / \mathrm{ml}$ were observed. For low plasma r-hirudin concentrations up to $0.2 \mu \mathrm{g} / \mathrm{ml}$, including the majority of the investigated patients, the ECT may not be reliable for guiding anticoagulation during CRRT. It is not clear whether this is related to methodological problems due to laboratory determinations or to interference with pathological conditions of other coagula- 
tion disorders. However, a more valid monitoring for $r$ hirudin based anticoagulation in continuous renal replacement therapy is required.
Reference:

1. Nowak G et al:: Seminars in Thrombosis and Hemostasis 1997, 22:197-202.

\section{Impact of CVVH on monocyte activation in the critically ill}

\section{R Rokyta, M Holub*, M Matejovic, J Hanzlikova, I Novak, P Hora, K Balihar and V Sramek}

ICU, Medical Department I, Charles University Hospital, Pilsen; *Department of Infectious Diseases, University Hospital Bulovka, Prague

Introduction: Monocyte activation (HLA-DR $\left.{ }^{+} \mathrm{CD} 14^{+}\right)$was suggested to be a useful marker of immune system function. As extracorporeal circuit might influence the immune response (e.g. bioincompatibility and/or elimination of pro/anti-inflammatory cytokines) we investigated the impact of CVVH initiation on monocyte activation in critically ill patients.

Methods: Fifteen septic (4 ICU survivors) and 7 nonseptic (1 ICU survivor) critically ill patients in whom CVVH (ultrafiltration 1-2 $\left.\mid \mathrm{h}^{-1}\right)$ was initiated because of acute renal failure as a part of MODS. Expression of HLA-DR+ CD14 ${ }^{+}$ was assessed by flow-cytometry before CVVH initiation and at $4 \mathrm{~h}$ and $24 \mathrm{~h}$ afterwards. The Friedmann two-way ANOVA and Mann-Whitney $U$ tests were used when appropriate. Data are presented as means $\pm S D ; P<0.05$ was considered significant.

Results: Baseline HLA-DR ${ }^{+} \mathrm{CD} 14^{+}$values in septic and nonseptic patients were not different $(57 \pm 21 \%$ and $71 \pm 21 \%$ respectively, $P=0.23)$. Time course of $\mathrm{HLA}-\mathrm{DR}{ }^{+} \mathrm{CD} 14^{+}$ during the first $24 \mathrm{~h}$ of $\mathrm{CVVH}$ are shown in Fig. 1.
Figure 1

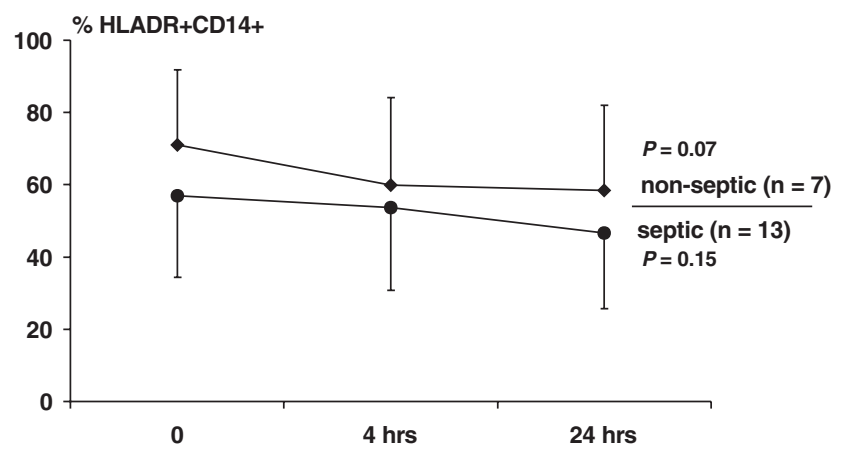

Conclusion: CVVH initiation has no impact on monocyte HLA-DR expression in both septic and nonseptic patients.

Acknowledgement: Supported by a Grant of IGA MZ (4972-3).

P38 Effect of continuous hemofiltration (CVVHF) on inflammatory parameters

\section{KH Smolle, W Wonisch, F Tatzber, P Kaufmann, G Koschsorur and R Aigner}

Introduction: Hemofiltration and hemodialysis are very important life saving procedures in the management of critically ill patients. The ability of these techniques to remove low molecular weight toxic substances from the circulation is very well documented, but little is known about their influence on inflammatory or lipid peroxidation parameters. Procalcitonin, PMN-elastase and Neopterin can be used as diagnostic and prognostic parameters to monitor critically ill patients and to control therapeutic interventions. For these reasons the aim of this preliminary investigation was to evaluate the effect of continuous veno-venous hemofiltration (CVVHF) versus intermittent hemodialysis (IHD) on plasma concentrations of inflammatory (Neopterin, PMN-elastase, Procalcitonin) and lipid peroxidation parameters (Malonic dialdehyde, Peroxides, Antibodies to oxidised LDL).

Patients and methods: We investigated 20 consecutive mechanically-ventilated critically ill patients $(n=12,25-86$ years, 7 male, 5 female) under CVVHF (blood flow
100-150 $\mathrm{ml} / \mathrm{min}$, ultrafiltrate 1-2 l/h). Cellulose triacetate membranes (FB-21U Biorena) $(n=3)$ and polyacrilonitrile membranes (AN69 Hospal) $(n=9)$ were used in this group. In the IHD group ( $n=8,31-86$ years, 5 male, 3 female) (blood flow 200-250 ml/min, dialysate flow $500 \mathrm{ml} / \mathrm{min}$ ) polysulfone membranes (F8HPS Fresenius) were applied. Blood samples were drawn at 0, 10,60 and 240 min after starting the procedure from before and after the membrane. Serum and plasma samples were drawn at 0, 10,60 and $240 \mathrm{~min}$ after the onset of CVVHF or IHD. After $30 \mathrm{~min}$ at $4^{\circ} \mathrm{C}$, the blood samples were centrifuged at $3000 \times \mathrm{g}$.

Malonic dialdehyde (MDA) determination was done by HPLC according to the method of Wong et al Neopterin (NPT), Elastase, Procalcitonin (PCT) and antibodies to oxidised LDL (oLAb) were determined by commercially available ELISA methods. Determination of peroxides was performed by an enzymatic method based on the peroxidase reaction with tetramethylbenzidine as a chromogenic substrate. 
Table 1

\begin{tabular}{|c|c|c|c|c|c|c|c|c|}
\hline Parameter & 0 pre & 0 post & 10 pre & 10 post & 60 pre & 60 post & 240 pre & 240 post \\
\hline \multicolumn{9}{|c|}{ CVVHF $(n=10)$} \\
\hline MDA & $1.60 \pm 0.73$ & $1.79 \pm 0.84$ & $1.61 \pm 0.78$ & $2.00 \pm 0.99$ & $1.60 \pm 0.56$ & $1.87 \pm 0.66$ & $1.60 \pm 0.47$ & $1.74 \pm 0.71$ \\
\hline Peroxide ${ }^{1}$ & 0.00 & 0.00 & 0.00 & 0.00 & 0.00 & 0.00 & 0.00 & 0.00 \\
\hline oLAb & $326 \pm 120$ & $373 \pm 160$ & $328 \pm 136$ & $381 \pm 203$ & $364 \pm 140$ & $388 \pm 181$ & $355 \pm 176$ & $395 \pm 214$ \\
\hline Elastase & $195 \pm 133$ & $240 \pm 157$ & $214 \pm 141$ & $254 \pm 144$ & $210 \pm 133$ & $256 \pm 136$ & $210 \pm 127$ & $262 \pm 151$ \\
\hline $\mathrm{PCT}^{2}$ & $12.6 \pm 11.3$ & $8.31 \pm 7.93$ & $10.4 \pm 8.26$ & $8.91 \pm 7.30$ & $10.7 \pm 9.98$ & $9.43 \pm 8.58$ & $10.4 \pm 9.82$ & $9.72 \pm 9.81$ \\
\hline Neopterin $^{3}$ & $103 \pm 18.8$ & $77.7 \pm 25.1$ & $108 \pm 32.8$ & $81.1 \pm 14.0$ & $110 \pm 32.0$ & $83.1 \pm 17.1$ & $100 \pm 24.4$ & $83 \pm 18.8$ \\
\hline \multicolumn{9}{|l|}{ IHD $(n=5)$} \\
\hline MDA & $1.39 \pm 0.59$ & $1.08 \pm 0.66$ & $1.68 \pm 0.89$ & $1.72 \pm 0.95$ & $1.54 \pm 0.73$ & $1.49 \pm 0.85$ & $1.31 \pm 0.54$ & $1.17 \pm 0.48$ \\
\hline Peroxide $^{1}$ & $160 \pm 126$ & $370 \pm 187$ & $264 \pm 175$ & $398 \pm 208$ & $247 \pm 179$ & $432 \pm 213$ & $329 \pm 181$ & $485 \pm 203$ \\
\hline oLAb & $265 \pm 182$ & $236 \pm 167$ & $244 \pm 127$ & $229 \pm 85.5$ & $254 \pm 160$ & $273 \pm 164$ & $236 \pm 167$ & $278 \pm 178$ \\
\hline Elastase & $114 \pm 86.2$ & $109 \pm 41.7$ & $132 \pm 86.1$ & $147 \pm 73.1$ & $176 \pm 89.4$ & $211 \pm 87.9$ & $179 \pm 71.1$ & $216 \pm 60.3$ \\
\hline $\mathrm{PCT}^{2}$ & $9.13 \pm 7.88$ & $7.30 \pm 6.37$ & $9.61 \pm 8.14$ & $9.50 \pm 8.07$ & $8.17 \pm 6.40$ & $8.86 \pm 6.92$ & $9.72 \pm 7.19$ & $10.3 \pm 8.20$ \\
\hline Neopterin ${ }^{3}$ & $131 \pm 72.5$ & $34.9 \pm 24.2$ & $124 \pm 59.9$ & $44.2 \pm 18.1$ & $101 \pm 45.8$ & $28.4 \pm 18.6$ & $93.4 \pm 40.0$ & $24.7 \pm 12.8$ \\
\hline
\end{tabular}

1, Median (No Gauss distribution of data); 2, Values >50 eliminated; 3, >10 and <500 included. Pre, Concentration before the membrane; Post, Concentration after the membrane.

Statistical analysis: In the case of a Gaussian distribution the statistical analysis was done by the t-test. Other distributions were assayed by the $\chi^{2}$ test.

Results: PCT and oLAb remained more or less constant in all observations. No statistically significant differences were observed before and after membrane passage or between the CVVHF and the IHD group. PMN-elastase concentrations increased constantly from start to the end of CVVHF and IHD. MDA levels reached the maximum 10 min after the onset of CVVHF and IHD. Again lower concentrations were observed in the IHD group compared with CVVHF. Peroxides were not detectable in most of the samples of the CVVHF group. In the IHD group, there were statistically significant differences in peroxide concentrations before and after the membrane passage. Neopterin concentrations decreased significantly after membrane passage in both CVVHF and IHD group. The decrease was more pro- nounced in the IHD group. Details in data for all parameters in both groups are presented in Table 1.

Discussion: For inflammation parameters (oLAb, NPT, PCT) no significant increases in concentrations could be observed. This indicates that no pronounced inflammatory reactions arise due to the dialysis procedure. The decrease in Neopterin concentrations after membrane passage shows efficient clearance of low molecular weight substances during CVVHF and IHD. In contrast, PMN-elastase concentrations increased from start to the end of CVVHF and IHD thus indicating PMN activation rather than activation of macrophages. In case of lipid peroxidation parameters, we found a peak in MDA concentrations 10 min after onset of CVVHF and IHD, which might be a consequence of PMN activation after contact with the membranes. In conclusion, our data suggest that even beneficial procedures like CVVHF or IHD may activate inflammatory and lipid peroxidation processes.

\section{P39 Prospective randomized study of hemodialysis membrane biocompatibility in acute renal failure}

A Jörres*, GM Gahl*, C Dobis*, A Kahl*, R Schindler*, U Frei*, C Guenther†, C Grossmann, W Gaus ${ }^{\ddagger}$ and J Hoegel ${ }^{\star}$ *Nephrology and Medical Intensive Care, UK Charité, Campus Virchow-Klinikum, Augustenburger Platz 1, D-13353 Berlin, Germany; ${ }^{\dagger}$ Membrana GmbH, Wuppertal; ${ }^{\ddagger}$ Biometry and Medical Statistics, University of Ulm, Germany

Introduction: There is considerable controversy in the current literature as to whether HD membrane biocompatibility may influence the mortality of patients with acute renal failure (ARF). We performed a prospective randomized (central telephone randomization) study in patients with dialysis-dependent ARF treated either with Cuprophan ${ }^{\circledR}$ (CUPRO) or polymethylmethacrylate (PMMA) lowflux membranes. The data presented are the unicentric evaluation of a larger international multicenter trial that was published recently [1]. 
Methods: In the study center in Berlin, a total of 104 patients were randomized. Of these, 94 patients $(35 f / 59 \mathrm{~m})$ with ARF were evaluable (CUPRO 45, PMMA 49 patients). Forty-six (49\%) of these were surgical/trauma patients. Mean age was 63.6 (19-87) years, mean APACHE II score was 26.3 (11-42). Sixtyseven $(71 \%)$ patients required mechanical ventilation, 81 $(86 \%)$ received parenteral nutrition.

Results: Overall, 56 patients (60\%) survived, 25 (56\%) in the CUPRO group and $31(63 \%)$ in the PMMA group $(P=$ n.s., Fisher's two-sided Exact Test). The odds ratio (OR) for not surviving for CUPRO vs PMMA was 1.38 with a $95 \% \mathrm{Cl}$ ranging from 0.56 to 3.42 . Moreover, no differ- ence between CUPRO and PMMA was detected when age and APACHE II score were entered as possible confounders in a logistic regression model. There was also no difference between the two study groups regarding time on dialysis, number of dialysis sessions required, need for mechanical ventilation, or total parenteral nutrition.

Conclusion: In summary, this controlled, prospective randomized trial did not reveal any differences in the outcome of dialysis-dependent ARF patients treated with CUPRO vs PMMA dialyser membranes.

References:

1. Jörres et al.: Lancet 1999, 354:1337-1341.

P40 Carbon dioxide elimination during continuous venovenous hemodiafiltration (CVVHD)-laboratory experiment

J Ruzicka, I Novak, R Rokyta, M Matejovic, P Hora, M Hadravsky and V Sramek

ICU and Department of Biophysics, Medical Faculty of Charles University, Pilsen, Czech Republic; Department of Biophysics, Karlovarska 36, Pilsen 301 66, Czech Republic

Objective: In a previous experiment with crystalloid solution we have shown that during $\mathrm{CVVH}(\mathrm{D}) \mathrm{CO}_{2}$ losses are proportional to the fractional ultrafiltration (UF) rate of circulating solution, with only a marginal impact of dialysis and temperature [1]. We report here results of a similar experiment with packed RBC.

Methods: Packed RBC were heparinized, diluted with crystalloid solution with bicarbonate to hematocrit of 0.35 and continuously saturated in a special mixing chamber to targeted $\mathrm{pCO}_{2}$ value of $5 \mathrm{kPa}$. After equilibration, the mixing chamber was connected to a PRISMA monitor (Prisma, Hospal, France) at a blood rate $(\mathrm{Ob})$ of $150 \mathrm{ml} \mathrm{hr}^{-1}$ and different CVVH(D) settings were tested for $\mathrm{CO}_{2}$ elimination at two temperature levels (37 and $40^{\circ} \mathrm{C}$ ). Blood samples were drawn in triplicate at each setting before and after the filter for blood gases analysis (ABL 520, Radiometer, Denmark). $\mathrm{CO}_{2}$ content was calculated using standard formula [2] and $\mathrm{CO}_{2}$ removal was expressed as pre-postfilter $\mathrm{CO}_{2}$ content difference.

Results: $\mathrm{CO}_{2}$ removal values (in \% of prefilter $\mathrm{CO}_{2}$ content) are summarised in Table 1 (means).

Conclusion: The often used CVVH setting (UF $1000 \mathrm{ml} / \mathrm{hr}$, $\mathrm{Ob} 150 \mathrm{ml} / \mathrm{min}$ ) leads to $\mathrm{CO}_{2}$ elimination at about
Table 1

\begin{tabular}{|c|c|c|c|c|c|}
\hline \multicolumn{3}{|c|}{ Blood temperature $37^{\circ} \mathrm{C}$} & \multicolumn{3}{|c|}{ Blood temperature $40^{\circ} \mathrm{C}$} \\
\hline & UF 1000 & UF 3000 & & UF 1000 & UF 3000 \\
\hline Dial 0 & $4.2 \%$ & $6.8 \%$ & Dial 0 & $5.2 \%$ & $11.0 \%$ \\
\hline Dial 1000 & $7.4 \%$ & $9.6 \%$ & Dial 1000 & $7.0 \%$ & $13.0 \%$ \\
\hline Dial 2500 & $10.7 \%$ & $13.3 \%$ & Dial 2500 & $9.4 \%$ & $15.4 \%$ \\
\hline
\end{tabular}

Dial-dialysis $[\mathrm{ml} / \mathrm{hr}]$, UF-ultrafiltration $[\mathrm{ml} / \mathrm{hr}]$

$5 \mathrm{ml} / \mathrm{min}$, i.e. a negligible part of the whole body $\mathrm{CO}_{2}$ production $\left(\mathrm{VCO}_{2}\right)$. A significant $\mathrm{CO}_{2}$ loss (about $15 \%$ of $\mathrm{VCO}_{2}$ ) can be expected using high volume ultrafiltration techniques.

Acknowledgement: This paper was supported by GAUK, Czech Republic.

References:

1. Ruzicka J, Šrámek V, Novák I, Hadravský M, Rokyta R, Hora P, Matejovic M, Nalos M: Gas exchange during continuous extracorporeal elimination techniques (CRRT). In Anaesthesia, Pain, Intensive Care and Emergency Medicine Selected Papers. Edited by Gullo A. 1998, p409-412.

2. Giovannini I, Chiarla C, Boldrini G, Castagneto M: Calculation of venoarterial $\mathrm{CO}_{2}$ difference. J Appl Physiol 1993, 74:959-964.

P41 The combination of lactate and bicarbonate buffers in continuous venovenous hemodiafiltration and its impact on serum lactate levels and homeostasis

M Balik*, A Kazda', M Kolar* and A HendI'

*Department of Intensive Care and Anaesthesiology, University Hospital Kralovske Vinohrady, Prague 10, Srobarova 50, Czech Republic; ${ }^{\dagger}$ Department of Clinical Biochemistry, Postgraduate Medical School, Faculty Policlinic, Prague, Czech Republic

Introduction: The buffer substitution of dialysis and hemofiltration is usually performed using either lactate or bicarbonate solutions. Bicarbonate works as a diuretic and it has certain renal tubular protective properties. The 
use of lactate containing solutions can interfere with the monitoring of lactate as a tissue oxygenation parameter.

Methods: Twelve septic ventilated patients were treated with continuous venovenous hemodiafiltration (CVVHDF). They were monitored before the treatment was started and after 24 and $48 \mathrm{~h}$. The dialysis fluid contained $35 \mathrm{mmol} / \mathrm{l}$ of Na-lactate as a buffer. The hemofiltration substitution fluid was Ringer's solution and $8.4 \%$ bicarbonate was infused at the average rate of $16.0 \mathrm{ml} / \mathrm{h}$. The dose was calculated initially and it was adjusted later to reach positive BE and mild hypernatremia up to $150 \mathrm{mmol} / \mathrm{l}$. In all patients the dose of furosemide was reduced to $1 \mathrm{mg} / \mathrm{kg}$.day after commencing of the treatment. Hemodynamic monitoring was performed with transesophageal echocardiography in all patients. Systemic oxygen delivery $\left(\mathrm{DO}_{2}\right)$ was calculated, oxygen extraction was approximated according to central venous $\mathrm{pO}_{2}\left(\mathrm{cvpO}_{2}\right)$.

Results: Lactate levels were found to be significantly elevated $24 \mathrm{~h}$ and $48 \mathrm{~h}$ after commencing the treatment: $1.77 \pm 0.68 \mathrm{mmol} / \mathrm{l}$ vs $2.70 \pm 0.88 \mathrm{mmol} / \mathrm{l}(P<0.001) \mathrm{vs}$ $2.52 \pm 1.06 \mathrm{mmol} / \mathrm{l}(P<0.03)$. No significant relationship was found between arterial lactate and $\mathrm{cvpO}_{2}$ before the treatment, but there was an indirect relationship between arterial lactate and $\mathrm{cvpO}_{2}$ during CVVHDF (correl. coeff. $r=-0.5 P=0.01 n=23$ ). The regression equation of the estimate of arterial lactate using $\mathrm{cvpO}_{2}$ was derived: lactate $=6.9-0.81^{*} \mathrm{CvpO}_{2}$. Average $\mathrm{DO}_{2}$ changed little $(777.2 \pm 187.0 \mathrm{ml} / \mathrm{min}$ vs $741.5 \pm 171.8 \mathrm{ml} / \mathrm{min}$ vs $760.1 \pm 149.6 \mathrm{ml} / \mathrm{min})$. Average sodium did not change but the standard deviation of sodium levels decreased (147.6 \pm 11.4 vs $148.7 \pm 6.9$ vs $146.2 \pm 1.9$ ) as well as the standard deviation of base excess (0.83 \pm 5.29 vs $2.24 \pm 4.00$ vs $1.74 \pm 3.48)$. Eight patients (67\%) had preserved sufficient residual renal functions. The average time of CVVHDF was 3.83 (3-5) days in six patients who survived (50\%).

Conclusion: Lactate remains a valuable marker of tissue hypoxia in patients treated with CVVHDF with constant input of lactate by dialysis. The stabilization of serum tonicity and acid-base balance was recorded. Continuous bicarbonate infusion during early CVVHDF can probably reduce the dose of other diuretics and promote the residual renal functions.

P42 Efficacy of the endotoxin absorption method (PMX) in patients with septic shock associated with intraperitoneal infections

T Ikeda, K Ikeda, T Onizuka, H Suzuki, K Suzuki and H Maruya

Division of Critical Care and Emergency Medicine, Tokyo Medical Center of Tokyo Medical University, 1163 Tatemchi, Hachiojicity, Tokyo, 193 Japan

Many points remain to be clarified concerning the mechanism of the endotoxin absorption method (PMX). In the present study, we investigated the efficacy of PMX in 13 patients who met all of the following four conditions: (1) patients in septic shock associated with an intraperitoneal infection; (2) patients who underwent SIRS; (3) patients with endotoxin (ET) levels of at least $10 \mathrm{pg} / \mathrm{ml}$ at the start of PMX; and (4) patients with circulatory dynamics requiring administration of catecholamine. The 13 patients consisted of nine men and four women with an average age of $62 \pm 10$ years. The outcome after four weeks was survival of nine patients and death of four patients. PMX was performed for $2 \mathrm{~h}$ and nafamosat mesylate was used as anticoagulant. The ET value, blood pressure, WBC, $\mathrm{pH}, \mathrm{PaO}_{2} / \mathrm{FiO}_{2}$, amount of DOA used, IL-6, IL-1ra, NOx, PAl-1, thrombo- modulin (TM), ICAM-1 and ELAM-1 were measured immediately before, immediately after and $24 \mathrm{~h}$ after PMX.

Results: The ET value showed a definite decrease from $29.7 \pm 23.4$ to $14.9 \pm 11.2 \mathrm{pg} / \mathrm{ml}$. The mean blood pressure increased from $79.7 \pm 13.8$ to $97.4 \pm 16.8$ torr. Among the cytokines, no significant differences were observed in IL-6 before and after PMX but IL-1 ra tended to decrease. PAI1 also decreased after PMX in the same way as IL-1ra. NOx, ICAM-1, ELAM-1 and TM showed no changes.

Conclusion: The inhibition of excessive increases in antiinflammatory cytokines by PMX was considered beneficial for the body's defense from the standpoint of preventing progression to CARS.

P43 Effects of epinephrine and norepinephrine on endotoxin-induced tissue factor expression in blood monocytes

\author{
L de Rossi, T Scholz*, T Eckermann+, U Temmler*, S Rußwurm+, K Reinhart' and W Lösche* \\ Clinic of Anaesthesiology, Rheinisch-Westfälische Technische Hochschule Aachen, Pauwelsstr. 30, D-52074 Aachen, \\ Germany; ${ }^{+}$Clinic of Anaesthesiology and Intensive Care Therapy; and *Centre for Vascular Biology and Medicine/Erfurt, \\ Friedrich-Schiller-University, Bachstr. 18, D-07740 Jena, Germany
}

Background: Tissue factor (TF) is the most important initiator of intravascular coagulation. In monocytes the expression of pro-inflammatory cytokines and TF is con- trolled by the same transcription factors. Catecholamines, frequently used in sepsis therapy of critically ill patients, have been shown to inhibit endotoxin-induced expression 
of monocyte cytokines, such as TNF- $\alpha$ or IL-6 [1]. The aim of our study was to prove whether epinephrine (EPI) and norepinephrine (NOREPI) may also affect TF expression.

Methods: To induce TF and TNF- $\alpha$ expression in monocytes we incubated citrated human whole blood (WB) for four hours with LPS (E. coli 055:B5; $50 \mathrm{ng} / \mathrm{ml})$. TF expression on monocytes was determined by flow cytometry, TNF- $\alpha$ secretion was measured by ELISA.

Results: Incubating WB samples with $50 \mathrm{ng} / \mathrm{ml}$ LPS we observed an increase in the number of TF-positive monocytes from $3.9 \pm 0.7 \%$ to $42.9 \pm 2.8 \%$. $5.5 \mathrm{nM}$ EPI inhibited LPS-induced TF expression by about $16 \%(P=0.03)$, but the inhibitory effect was attenuated at higher EPI concentrations $(>550 \mathrm{nM})$. LPS-induced TNF- $\alpha$ secretion $(4.2 \pm 0.5 \mathrm{ng} / \mathrm{ml})$ was inhibited by about $60 \%$ in presence of $5.5 \mathrm{nM} \mathrm{EPI}$, and maximum inhibition of $75 \%$ was reached at $55 \mathrm{nM}$. Compared to EPI, higher concentrations of NOREPI were needed to get a significant inhibition of monocyte activity. Inhibition of TF-expression by $13 \%$ was observed at $550 \mathrm{nM}$ and the same inhibition was observed at $5,500 \mathrm{nM}$. TNF- $\alpha$ secretion was inhibited in a clear dose-dependent manner and amounted at 5.5 nM NOREPI to about $35 \%$ and at $5500 \mathrm{nM}$ to about $75 \%$.

Conclusion: EPI is more effective than NOREPI to inhibit LPS-induced monocyte TF expression and TNF- $\alpha$ secretion. At higher concentrations EPI also seemed to have a stimulatory effect on TF expression, which could be due to an interaction of EPI-activated platelets [2] with monocytes.

\section{References:}

1. van der Poll et al.: Infect Immunol 1994, 62:2046-2050.

2. Amirkhosravi et al:: Thromb Haemost 1996, 75:87-95.

\title{
P44 Influence of enoximone and dobutamine upon liver perfusion and function in fluid optimized septic patients
}

\author{
H Kern, T Schröder, M Kaulfuss, WJ Kox and C Spies \\ Department of Anesthesiology and Intensive Care Medicine, University Hospital Charité (Campus Mitte), Humboldt-University \\ Berlin, Schumannstr. 20/21, 10117 Berlin, Germany
}

Introduction: Splanchnic mucosal perfusion abnormalities have been implicated in the development of sepsis and multiple organ failure. In clinical and experimental settings, administration of dobutamine can increase hepatosplanchnic perfusion $[1,2]$. The administration of enoximone during and after cardiopulmonary bypass diminished endotoxin levels in liver venous blood indicating a beneficial effect on tissue perfusion and barrier function of the gut [3]. The aim of this study was to investigate the impact of dobutamine and enoximone upon liver perfusion and function in fluid optimized septic patients.

Methods: After approval by the local ethical committee and obtained written informed consent by next of kin, 48 septic patients were included in this study. After fluid resuscitation according to an optimal left atrial filling pressure established by plotting left ventricular stroke work index against pulmonary artery occlusion pressure, patients were randomly treated with dobutamine (initially $5 \mu \mathrm{g} / \mathrm{kg} / \mathrm{min}$, increasing dose up to a maximum of $20 \mu \mathrm{g} / \mathrm{kg} / \mathrm{min}$ ) or enoximone (initially $2.5 \mu \mathrm{g} / \mathrm{kg} / \mathrm{min}$, increasing dose up to a maximum of $10 \mu \mathrm{g} / \mathrm{kg} / \mathrm{min}$ ) for $10 \mathrm{~h}$ up to a dosage where no further increase in left ventricular stroke work index was achieved. Hemodynamics, liver blood flow (LBF), hepatic tumor necrosis factor (TNF) and monoethylglycinexylidide (MEGX) kinetics to assess hepatic function were performed within the first $12 \mathrm{~h}$ of sepsis, before administration of inotropic support as well as $12 \mathrm{~h}$ and $48 \mathrm{~h}$ after treatment. Statistical analysis was performed using Wilcoxon signed rank sum and Friedman test.

Results: Basic patient characteristics (age, sex, APACHE III) did not differ between groups. Oxygen delivery and oxygen consumption increased in both groups without significant difference between groups.

Conclusion: Administration of enoximone in fluid optimized septic patients may be favorable in comparison to dobutamine. Enhanced $\mathrm{DO}_{2}$ and $\mathrm{VO}_{2}$ in both regimens resulted in an increased regional perfusion and improved splanchnic function only in the enoximone group indicated

\begin{tabular}{|c|c|c|c|c|c|c|}
\hline & \multicolumn{3}{|c|}{ Enoximone } & \multicolumn{3}{|c|}{ Dobutamine } \\
\hline & Baseline & $12 \mathrm{~h}$ treatment & $48 \mathrm{~h}$ treatment & Baseline & $12 \mathrm{~h}$ treatment & $48 \mathrm{~h}$ treatment \\
\hline $\mathrm{LBF}\left[\mathrm{L} / \mathrm{min} / \mathrm{m}^{2}\right]$ & $2.9 \pm 0.7$ & $3.3 \pm 0.8 b$ & $3.2 \pm 0.7 \mathrm{~b}$ & $2.8 \pm 1.0$ & $3.0 \pm 0.8 b$ & $2.9 \pm 0.8 \mathrm{~b}$ \\
\hline LTNF [pg/mL] & $32 \pm 26$ & $43 \pm 31 \mathrm{ab}$ & $37 \pm 32$ & $29 \pm 28$ & $62 \pm 36 a b$ & $29 \pm 26$ \\
\hline MEGX $[\mu \mathrm{g} / \mathrm{L}]$ & $19 \pm 19$ & $33 \pm 26 a b$ & $29 \pm 20 \mathrm{~b}$ & $22 \pm 16$ & $21 \pm 22 \mathrm{a}$ & $32 \pm 17 b$ \\
\hline
\end{tabular}

Results are presented as means \pm standard deviation; a) $P<0.05$ between groups; b) $P<0.05$ within groups compared to baseline. 
by a diminished release of the pro-inflammatory hepatic TNF and increased MEGX.
References:

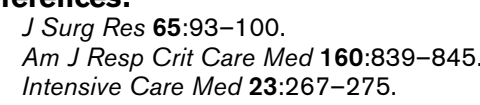

\section{P45 Renal blood flow in critically ill cardiac patients: effect of dobutamine on total flow and selective redistribution of the cardiac index}

\section{H Sherif, AW Andraos, A Abdel Fattah, A Amer, A Eltaweel, O Elhennawy and MS Mokhtar}

Baseline regional distribution of cardiac output (CO) depends on the physiological determinants of the endorgans. In congestive heart failure (CHF), this distribution is modified in favour of the coronary, brain and kidney circulations, depending on the main regulatory mechanisms of the vasomotor system, including the sympathetic nervous system, the vascular endothelium and the local non-endothelial mechanisms.

In this study, we measured the renal blood flow (RBF) and $\mathrm{CO}$ in 20 critically ill cardiac patients (17 males, mean age $49 \pm 10$ years), utilizing both techniques of color coded duplex and echocardiography. All patients had CHF due either to dilated cardiomyopathy (12 patients) or ischemic heart disease (8 patients). A control group of 14 males (mean age $41 \pm 8.6$ years) was also included. Following clinical evaluation, including the history and 12-lead ECG, all patients and control group were subjected to duplex assessment of RBF $\left(\mathrm{ml} / \mathrm{min} / \mathrm{m}^{2}\right)$, in post-absorptive state by measuring the internal diameters of both renal arteries at their aortic origins, and then estimation of the renal artery pulsed flow wave to measure the time average velocity, (the average of the velocity spectrum in one second) and then multiplied by 60 to express the flow in one min. The CO was measured echocardiographically through the pulsed wave Doppler trans-aortic flow. The whole procedure was repeated, first under low dose $(5 \mu \mathrm{g} / \mathrm{kg} / \mathrm{min})$, and then under high dose $(20 \mu \mathrm{g} / \mathrm{kg} / \mathrm{min})$ dobutamine, with $24 \mathrm{~h}$ apart. The regional renal percentage (RRP) was calculated and expressed as percentage of the cardiac index.

Compared to the control group, patients, at baseline, showed statistically significant lower $\mathrm{CO}, \mathrm{RBF}$ and RRP (mean $=16.7 \pm 4.3 \%$ vs $19.3 \pm 4.1 \%, P<0.003$ ). On low dose dobutamine, they showed a significantly higher $\mathrm{CO}$ and RBF with an insignificant increase in RRP (mean $=17.3 \pm 2.3 \%$ ) due to renal vasodilatation. On the other hand, reaching the higher dose of dobutamine, CO showed a further statistically significant increase, whereas RBF and RRP decreased significantly (mean = $14.2 \pm 3.4 \%, P<0.02)$, due to renal vasoconstriction.

Conclusion: The effects of different doses of dobutamine on the $\mathrm{CO}$ were not paralleled by similar effects on the RBF and the RRP. In patients with CHF, the adjustment of the proper dose of dobutamine should follow the regional renal blood flow rather than the absolute values of the $\mathrm{CO}$.

P46 Influence of iloprost on hepato splanchnic metabolic activity and energy balance in patients with septic shock

P Kiefer, I Tugtekin, H Bracht, C Altin, J Vogt, H Wiedeck, M Georgieff and P Radermacher

Universitätsklinik f. Anästhesiologie, Universität, D-89070 Ulm, Germany

Introduction: Septic shock is characterized by increased splanchnic blood flow due to enhanced metabolic activity. Endogenous prostacyclin may be crucial to maintain liver function and gastric mucosal integrity [1]. Therefore we studied the effect of intravenous. iloprost on hepatosplanchnic metabolic activity and energy balance in patients with septic shock.

Patients/methods: Twelve patients with septic shock (cardiac index, $\mathrm{Cl} \geq 3 \mathrm{l} / \mathrm{min} \times \mathrm{m}^{2}$, all requiring noradrenaline $\geq 0.03 \mu \mathrm{g} / \mathrm{kg} \times \mathrm{min}^{-1}$ to maintain mean arterial pressure (MAP $\geq 70 \mathrm{mmHg}$ ) were studied. In addition to routine systemic hemodynamics and gas exchange we inserted a Swan Ganz catheter into a hepatic vein (hv) to measure splanchnic blood flow using primed continuous infusion of indocyanine-green. Moreover, we assessed splanchnic lactate uptake (Fick principle), endogenous glucose production rate (stable isotope approach), as well as hepatic venous lactate/pyruvate and acetoacetate $/ \beta-\mathrm{OH}$-butyrate ratios. Measurements obtained after 90' of hemodynamic steady-state were recorded before, after and during iloprost infusion titrated to obtain a $20 \%$ increase in $\mathrm{Cl}$ $\left(0.9-3 \mathrm{ng} / \mathrm{kg} \times \mathrm{min}^{-1}\right)$.

Results/statistics: See Table. Friedmann test/StudentNewman-Keuls: ${ }^{*} P<0.05$ vs baseline.

Conclusion: While maintaining hepato-splanchnic lactate clearance, iloprost reduced the endogenous glucose production rate, hence the hepatic $\mathrm{O}_{2}$ requirements. The unchanged regional $\mathrm{O}_{2}$ uptake therefore suggests shifting of $\mathrm{O}_{2}$ consumption to other energy demanding processes. 


\begin{tabular}{lccc} 
(median/percentile) & Baseline & lloprost & Baseline \\
\hline Sys. $\mathrm{DO}_{2} \mathrm{ml} / \mathrm{min} / \mathrm{m}^{2}$ & $565 / 642-473$ & $634 / 699-513$ & $524 / 651-472$ \\
Sys. $\mathrm{VO}_{2} \mathrm{ml} / \mathrm{min} / \mathrm{m}^{2}$ & $139 / 142-131$ & $147 / 164-136^{*}$ & $143 / 154-133$ \\
$\mathrm{Spl.} \mathrm{DO}_{2} \mathrm{ml} / \mathrm{min} / \mathrm{m}^{2}$ & $122 / 166-103$ & $134 / 203-117^{*}$ & $130 / 158-98$ \\
$\mathrm{Spl} . \mathrm{VO}$ & $\mathrm{ml} / \mathrm{min} / \mathrm{m}^{2}$ & $59 / 71-57$ & $56 / 65-52$ \\
Spl. Lactate balance $\mu \mathrm{mol} / \mathrm{kg} / \mathrm{min}$ & $58 / 67-49$ & $13 / 14-2$ & $11 / 13-5$ \\
Glucose production $\mu \mathrm{mol} / \mathrm{kg} / \mathrm{min}$ & $12 / 13-4$ & $11 / 15-9^{*}$ & $11 / 11-8$ \\
Hv. Lactate/pyruvate & $16 / 19-14$ & $16 / 34-12$ & $13 / 22-12$ \\
Hv. Acetoacetate//3-OH-butyrate & $18 / 21-16$ & $0.22 / 0.45-0.08$ & $0.22 / 0.34-0.05$ \\
\hline
\end{tabular}

Acknowledgement: Supported by Deutsche Forschungsgemeinschaft (Ra 396/4-1); Schering - Germany
Reference:

1. Brinkmann A et al.: Crit Care Med 1996; 24:1293-1301.

\section{P47 Epinephrine is more effective than other sympathomimetics in correcting cerebral hypoperfusion associated with mesenteric ischemic reperfusion insult}

MM Zayek, CR Hamm, KT O'Donnell and FG Eyal

Pediatrics, University of south Alabama, Mobile, AL, USA

Objective: To assess the efficacy of sympathomimetics in maintaining cerebral blood flow when cardiac function is impaired.

Background: Sympathomimetics are frequently used to treat hypotensive newborns with one of the goals being the preservation of cerebral blood flow. No animal model has substantiated the efficacy of this practice. Although inotropes have been extensively studied in healthy animals, little is available concerning their efficacy in "sick" hemodynamically impaired newborn animals.

Design/methods: A laparotomy was performed in anesthetized piglets $(10 \pm 1$ days old, $n=40$ ) to clamp a major branch of the superior mesenteric artery for $30 \mathrm{~min}$. One hour after, a persistent state of impaired cardiac function was produced. Cardiac output remained $24 \pm 2 \%$ below initial baseline. There was a parallel decrease in carotid blood flow $(21 \pm 5 \%)(\mathrm{CBF})$ while the decrement in systemic mean blood pressure (BP) was small $(73 \pm 2$ to $67 \pm 2 \mathrm{mmHg} ; P<0.01)$. Those parameters remained the same for the next two hours when treatment consisted only of intravenous normal saline at a rate of $35 \mathrm{ml} / \mathrm{kg} / \mathrm{h}$. One hour after the start of mesenteric reperfusion, animals were randomized to additionally receive: Dobutamine (DOB), Dopamine (DP), Epinephrine (EPI) or no inotropes (CONT). The sympathomimetics (SYMP) were given at $20 \mathrm{~min}$ intervals, using a randomized latin squares design, at a rate of 5, 10, 20, 40 (DOB and DP) or 0.5, 1, 2 and $4 \mu \mathrm{g} / \mathrm{kg} / \mathrm{min}$ (EPI). The animals were instrumented to measure: aortic, pulmonary artery and superior sagittal sinus blood gases and lactate, cerebral oxygen extraction (CBF ext); mean systemic and pulmonary artery pressures(PAP); cardiac output (thermodilution), carotid blood flow (ultrasonic flow transducers).

Results: See Table. Mean \pm s.e.m of the \% change from levels immediately prior to treatment.

Conclusions: Depressed cardiac output is associated with a significant decrease in cerebral blood flow even though BP is minimally reduced. EPI may be more efficient than the other inotropes in supporting neonatal CBF when $\mathrm{CO}$ is impaired.

\begin{tabular}{|c|c|c|c|c|c|c|c|c|c|}
\hline \multirow{2}{*}{$\begin{array}{l}\% \text { change } \\
\text { Rate }\end{array}$} & \multicolumn{3}{|c|}{ DOBUTAMINE } & \multicolumn{3}{|c|}{ DOPAMINE } & \multicolumn{3}{|c|}{ EPINEPHRINE } \\
\hline & 10 & 20 & 40 & 10 & 20 & 40 & 1 & 2 & 4 \\
\hline $\mathrm{CO}^{\star \star}$ & $28 \pm 2^{*}$ & $49 \pm 13$ & $44 \pm 13$ & $24 \pm 7^{\star}$ & $41 \pm 12$ & $51 \pm 20$ & $29 \pm 12^{*}$ & $42 \pm 12$ & $39 \pm 12$ \\
\hline $\mathrm{BP}$ & $-3 \pm 6$ & $-0.2 \pm 5$ & $-2.5 \pm 8$ & $0.4 \pm 3$ & $2 \pm 3$ & $1.5 \pm 4$ & $12 \pm 6^{\S}$ & $25 \pm 5^{\S}$ & $44 \pm 7^{\S}$ \\
\hline CBF & $-5 \pm 6$ & $10 \pm 7$ & $18 \pm 9$ & $-3 \pm 4$ & $5 \pm 6$ & $-2 \pm 10$ & $7 \pm 12$ & $26 \pm 8$ & $19 \pm 7$ \\
\hline CBF ext & $9 \pm 11$ & $3 \pm 7$ & $1 \pm 6$ & $-1 \pm 6$ & $-2 \pm 7$ & $9 \pm 7$ & $-11 \pm 6$ & $-23 \pm 6 \S$ & $-30 \pm 8^{\S}$ \\
\hline
\end{tabular}

${ }^{*}: 10 \neq 20,40$ or $1 \neq 2,4,{ }^{* *}:$ SYMP $\neq$ CONT, $\S:$ EPI $\neq$ CONT,DOB,DP; $P<0.05$; RM Anova. All SYMP similarly improved cardiac output. EPI was the

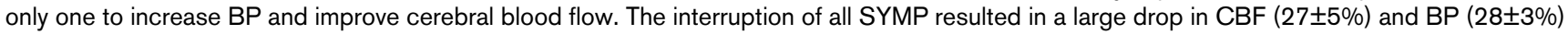
while $\mathrm{CO}$ remained unchanged. 
P48 Norepinephrine-induced blood pressure increase to pre-shock levels worsens regional flow distribution in porcine septic shock

MM Treggiari-Venzi, J-A Romand, D Burgener*, M Svensson ${ }^{\dagger \neq}$, PM Suter and A Aneman ${ }^{\ddagger}$

Surgical Intensive Care Unit Division, *Division of Gastroenterology, University Hospital, 1211 GENEVA 14-Switzerland;

${ }^{\dagger}$ Departments of Physiology, Göteborg University, and ${ }^{\ddagger}$ Anesthesiology and Intensive Care, Sahlgren’s University Hospital,

Göteborg, Sweden

Septic shock is characterized by hypotension, persisting after fluid replacement and requiring vasopressors. Mean arterial pressure (MAP) as endpoint of treatment providing adequate regional perfusion is unclear. In an acute endotoxic shock model, norepinephrine was used to reverse hypotension in seven fluid-resuscitated pigs, anesthetized with $\alpha$-chloralose and equipped with flow probes around the portal vein and renal artery, renal and jejunal mucosal laser Doppler flowmetry and jejunal tonometry. MAP was increased by 10 and $20 \mathrm{mmHg}$ above the shock level with norepinephrine. Seven shocked, fluid-resuscitated only animals served as control. Measurements were performed before 2 h-endo- toxin infusion and at the end of each increased MAP level. Raising MAP with norepinephrine by $10 \mathrm{mmHg}$ increased significantly cardiac output, systemic oxygen extraction, portal vein blood flow, improved metabolic balance and tended to restore renal and jejunal mucosal flows to preshock levels. Increasing MAP by $20 \mathrm{mmHg}$ further increased cardiac output and oxygen delivery but reduced portal vein blood flow and tended to decrease renal and jejunal mucosal flows. In conclusion, using norepinephrine to increase MAP by $10 \mathrm{mmHg}$ in volumeresuscitated, acutely septic animals improved systemic and regional perfusion. Higher MAP increase did not add any benefit despite an enhanced cardiac output.

P49 Inhibition of inducible nitric oxide synthase (iNOS) reduces multi-organ failure (MOF) in the thioacetamide (TAA) rat model

TM Rahman and HJF Hodgson

Centre for Hepatology, Department of Medicine, Royal Free School of Medicine, Rowland Hill Street, London NW1, UK

Introduction: The role of iNOS in MOF has been controversial, studies having presented contradicting results, however, the use of more specific inhibitors of iNOS may provide benefit.

Aim: To examine the effects of iNOS inhibition in our model of TAA induced MOF.

Method: MOF was induced by two intraperitoneal (IP) injections of TAA (500 mg/kg) eight hours apart. Three groups were studied, Group1 receiving TAA only. Group 2 and 3 followed thr protocol for Group 1, however, Group 2 was pre-treated with the NO precursor L-Arginine (300 mg/kg IP) once daily and Group 3 was pre-treated with NO synthase inhibitor aminoguanidine (100 mg/kg SC) for three days.

Results: See Table. $\mathrm{n}=10$ Mean $\pm S D$.

Histology: The histological sections show markedly less organ damage in the aminoguanidine group (Group 3).

Conclusion: Inhibition of iNOS using aminoguanidine significantly improves the incidence of MOF and mortality in the TAA model of MOF.

\begin{tabular}{|c|c|c|c|c|c|c|c|}
\hline \multirow{2}{*}{$\begin{array}{l}\text { Groups } \\
\text { Time (h) }\end{array}$} & \multicolumn{2}{|c|}{ AST (iu/l) } & \multicolumn{2}{|c|}{ Creatinine (umol/l) } & \multicolumn{2}{|c|}{ Lactate $\mathrm{mmol} / \mathrm{l}$} & \multirow{2}{*}{$\frac{\text { Survival \% }}{96}$} \\
\hline & 24 & 48 & 24 & 48 & 24 & 48 & \\
\hline Control & $34 \pm 10$ & $41 \pm 12$ & $43 \pm 13$ & $51 \pm 10$ & $1.5 \pm 0.3$ & $1.4 \pm 0.2$ & 100 \\
\hline TAA & $1653 \pm 164$ & $4045 \pm 247$ & $167 \pm 22$ & $231 \pm 37$ & $3.9 \pm 0.9$ & $6.2 \pm 0.7$ & 25 \\
\hline TAA + L-ARG & $2177 \pm 231$ & $4475 \pm 240$ & $187 \pm 14$ & $271 \pm 27$ & $4.2 \pm 0.5$ & $7.3 \pm 0.7$ & 10 \\
\hline $\mathrm{TAA}+\mathrm{AMG}$ & $346 \pm 50$ & $1090 \pm 188$ & $87 \pm 21$ & $112 \pm 23$ & $2.1 \pm 0.2$ & $3.4 \pm 0.3$ & 90 \\
\hline
\end{tabular}

\section{Effect of iNOS inhibition on exhaled NO and serum nitrate in hyperdynamic porcine endotoxemia}

MM Theisen, D Vasilev, J Altherr, M Matejovic*, I Tugtekin, M Georgieff and P Radermacher

Department of Anesthesia, University Hospital, D-89073 Ulm, Germany; *Department of Intensive Care, Charles-University, CZ30460 Plzen

Introduction: Controversial data have been reported on the pivotal role of excessive nitric oxide (NO) production for sepsis-induced vasodilatation in higher species $[1,2,3]$. Since both the measurement of plasma nitrate $\left(\mathrm{NO}_{3}\right)$ and 


\begin{tabular}{|c|c|c|c|c|c|}
\hline & Baseline & $12 \mathrm{~h}$ & $18 \mathrm{~h}$ & $24 \mathrm{~h}$ & \\
\hline \multirow[t]{2}{*}{ A nitrate $\mathrm{mol} / \mathrm{l}$} & CNT & $24(13 ; 31)$ & $21(16 ; 33)$ & $22(13 ; 31)$ & $18(11 ; 31)$ \\
\hline & $1400 \mathrm{~W}$ & $28(16 ; 29)$ & $21(17 ; 26)$ & $17(14 ; 23)$ & $15(13 ; 23)$ \\
\hline \multirow[t]{2}{*}{$\mathrm{PV}$ nitrate $\mathrm{mol} / \mathrm{l}$} & CNT & $20(11 ; 25)$ & $25(16 ; 28)$ & $23(13 ; 30)$ & $20(16 ; 28)$ \\
\hline & $1400 \mathrm{~W}$ & $18(14 ; 28)$ & $20(15 ; 22)$ & $19(13 ; 22)$ & $16(14 ; 18)$ \\
\hline \multirow[t]{2}{*}{$\mathrm{HV}$ nitrate $\mathrm{mol} / \mathrm{l}$} & CNT & $25(16 ; 34)$ & $24(15 ; 34)$ & $23(14 ; 32)$ & $21(10 ; 29)$ \\
\hline & $1400 \mathrm{~W}$ & $25(16 ; 28)$ & $19(16 ; 27)$ & $20(15 ; 23)$ & $18(14 ; 20)$ \\
\hline \multirow[t]{2}{*}{$\mathrm{NO}$ expired $\mathrm{pmol} / \mathrm{kg} / \mathrm{min}$} & CNT & $8(4 ; 12)$ & $25(19 ; 34)^{*}$ & $18(13 ; 25)^{\star}$ & $22(12 ; 27)^{\star}$ \\
\hline & $1400 \mathrm{~W}$ & $9(5 ; 16)$ & $36(27 ; 55)^{*}$ & $13(9 ; 18)$ & $17(12 ; 21)$ \\
\hline
\end{tabular}

exhaled NO levels are used as a surrogate for excess NO formation, we investigated the effect of selective iNOS inhibition on these parameters during longterm porcine endotoxemia [4].

Methods: $12 \mathrm{~h}$ after shock induction with continuous i.v. endotoxin (LPS) anesthetized pigs were randomly assigned to receive either LPS alone (CNT, $n=13)$ or LPS plus the selective iNOS inhibitor 1400W (1400W, $n=9$ ) tritrated to keep MAP at pre-shock levels (range 0.1-0.5 $\mathrm{mg} / \mathrm{kg} / \mathrm{h}$ ). Hydroethyl starch was infused to maintain sustained increase in cardiac output. Before, as well as 12, 18 and $24 \mathrm{~h}$ after, the start of LPS we measured expired $\mathrm{NO}$ levels and arterial (A), portal (PV) and hepatic venous (HV) $\mathrm{NO}_{3}$ concentrations (after chemical reduction to $\mathrm{NO}$ ) using chemiluminescence. Exhaled NO was calculated as described in [5].

Results: Data are median (25/75\% quartiles), $P<0.05$ *vs pre-shock (RM ANOVA on ranks, SNK test for multiple comparison), ${ }^{\dagger} 1400 \mathrm{~W}$ vs CNT (Mann-Whitney Rank Sum Test). There was no significant relationship between exhaled $\mathrm{NO}$ and blood $\mathrm{NO}_{3}$ concentration.

Conclusions: Remaining plasma $\mathrm{NO}_{3}$ levels do not reflect excess NO release during sepsis, probably due to variable volume of distribution resulting from interstitial edema and/or flux into third spaces such as ascites. Exhaled NO may be more appropriate for on-line monitoring of NO production in sepsis.

Acknowledgement: Supported by the Deutsche Forschungsgemeinschaft, Deutscher Akademischer Austauschdienst and ESICM.

\section{References:}

1. Šantak B et al.: Br J Pharmacol 1997, 122:1605-1610.

Pastor CM et al:: AJP 1999, in press.

Mehta S et al:: Crit Care Med 1999, 27:385-393.

Šantak B et al.: Br J Pharmacol 1998, 124:1689-1697.

Mehta S et al:: Chest 1997, 111:1045-1049.

\section{P51 Effects of mercaptoethylguanidine during long-term hyperdynamic porcine endotoxemia}

\section{F Ploner*, I Tugtekin, M Matejovic ${ }^{\dagger}$, M Theisen, A Stehrł, C Szabo§, UB Brückner\# and P Radermacher}

${ }^{*}$ Department of Anesthesia, District Hospital, I-39042 Brixen; ${ }^{\dagger}$ Department of Intensive Care, Charles-University, CZ-30460 Plzen; " Department of Surgery, University Hospital, D-93042 Regensburg; §Inotec Inc., Beverly, MA 01915, USA; Division of Pathophysiology in Anesthesia and "Surgical Research, University Hospital, D-89073 Ulm, Germany

Introduction: Excess NO production due to iNOS activation and cellular toxicity resulting from peroxynitrite $\left(\mathrm{ONOO}^{-}\right)$may contribute to organ dysfunction in septic shock. Therefore, we studied the effect of the combined $\mathrm{ONOO}^{-}$scavenger and selective iNOS-inhibitor mercaptoethylguanidine (MEG) [1] on hepato-splanchnic hemodynamics and energy metabolism during long-term hyperdynamic porcine endotoxemia [2].

Methods: $12 \mathrm{~h}$ after starting continuous intravenous endotoxin, pigs received either no drug (CNT; $n=9)$ or 3 $\mathrm{mg} / \mathrm{kg} / \mathrm{h}$ MEG ( $\mathrm{n}=7$ ). Hydroxyethyl starch was infused to maintain a sustained increase in cardiac output [2]. Before, as well as 12, 18, and $24 \mathrm{~h}$ after, the start of LPS we assessed expired NO formation (chemiluminescence), systemic (CO) and liver (Doppler ultrasound flow probes) blood flow, arterial-ileal mucosal $\mathrm{PCO}_{2}$-gap (fiberoptic sensor), portal (pv) and hepatic venous (hv) lactate/pyruvate $(\mathrm{L} / \mathrm{P})$ ratios and hepatic lactate clearance.

Results: See Table.

Conclusion: MEG allowed for hemodynamic stabilization due to blunting of the progressive endotoxin-induced fall in MAP while maintaining $\mathrm{CO}$ but did not influence the parameters of hepato-splanchnic energy metabolism. Ongoing oxidative stress resulting from inadequate dosage of the compound may account for this result [3].

Acknowledgement: Supported by Provinz Bozen-Südtirol (Italy), ESICM and Deutsche Forschungsgemeinschaft.

\section{References:}

1. Szabó et al.: Crit Care Med 1999, 27:1353.

2. Šantak et al: Br J Pharmacol 1998,124:1689-1697.

3. Whiteman et al: Br J Pharmacol 1999, 126:1646. 


\begin{tabular}{|c|c|c|c|c|c|c|}
\hline & & Before LPS & 12 h LPS & 18 h LPS & 24 h LPS & \\
\hline $\begin{array}{l}\text { Expir. NO } \\
\mathrm{pmol} / \mathrm{kg} / \mathrm{min}\end{array}$ & $\begin{array}{l}\text { CNT } \\
\text { MEG }\end{array}$ & $\begin{array}{c}6[2 ; 8] \\
7[6 ; 14]\end{array}$ & $\begin{array}{l}16[13 ; 21] \# \\
20[16 ; 34] \#\end{array}$ & $\begin{array}{c}13[10 ; 18] \# \\
8[6 ; 16]\end{array}$ & $\begin{array}{c}15[14 ; 19] \# \\
10[3 ; 19]\end{array}$ & $\begin{array}{l}\text { Data are median } \\
{[25 ; 75 \% \text { quartile] }}\end{array}$ \\
\hline $\begin{array}{l}\text { MAP } \\
\mathrm{mmHg}\end{array}$ & $\begin{array}{l}\text { CNT } \\
\text { MEG }\end{array}$ & $\begin{array}{c}101[91 ; 106] \\
99[90 ; 105]\end{array}$ & $\begin{array}{c}85[75 ; 95] \# \\
88[82 ; 97]\end{array}$ & $\begin{array}{c}79[68 ; 90] \# \\
94[90 ; 111] \S\end{array}$ & $\begin{array}{c}82[61 ; 85] \# \\
102[93 ; 106] \S\end{array}$ & $\begin{array}{l}\# P<0.05 \text { vs Before } \\
\text { LPS (Kruskal-Wallis }\end{array}$ \\
\hline $\begin{array}{l}\mathrm{CO} \\
\mathrm{ml} / \mathrm{kg}\end{array}$ & $\begin{array}{l}\text { CNT } \\
\text { MEG }\end{array}$ & $\begin{array}{c}108[98 ; 122] \\
115[104 ; 128]\end{array}$ & $\begin{array}{l}160[147 ; 168] \# \\
154[135 ; 165] \#\end{array}$ & $\begin{array}{l}150[128 ; 174] \# \\
159[136 ; 175] \#\end{array}$ & $\begin{array}{l}159[134 ; 198] \# \\
160[149 ; 186] \#\end{array}$ & $\begin{array}{l}\text { ANOVA on Ranks) } \\
\S P<0.05 \text { MEG vs }\end{array}$ \\
\hline $\begin{array}{l}\text { L/P ratio } \\
\text { pv }\end{array}$ & $\begin{array}{l}\text { CNT } \\
\text { MEG }\end{array}$ & $\begin{array}{l}15[13 ; 17] \\
13[11 ; 14]\end{array}$ & $\begin{array}{l}19[18 ; 21] \# \\
18[17 ; 21] \#\end{array}$ & $\begin{array}{l}20[17 ; 28] \# \\
21[18 ; 26] \#\end{array}$ & $\begin{array}{l}25[21 ; 30] \# \\
24[20 ; 27] \#\end{array}$ & $\begin{array}{l}\text { CNT (Mann- } \\
\text { Whitney Test). }\end{array}$ \\
\hline $\begin{array}{l}\mathrm{PCO}_{2} \text {-gap } \\
\mathrm{mmHg}\end{array}$ & $\begin{array}{l}\text { CNT } \\
\text { MEG }\end{array}$ & $\begin{array}{c}15[8 ; 17] \\
14[10 ; 22]\end{array}$ & $\begin{array}{l}17[15 ; 26] \\
22[17 ; 25]\end{array}$ & $\begin{array}{l}20[16 ; 27] \\
15[13 ; 20]\end{array}$ & $\begin{array}{l}20[10 ; 31] \\
14[10 ; 26]\end{array}$ & \\
\hline $\begin{array}{l}\text { Liver blood } \\
\text { flow } \mathrm{ml} / \mathrm{kg}\end{array}$ & $\begin{array}{l}\text { CNT } \\
\text { MEG }\end{array}$ & $\begin{array}{l}28[23 ; 31] \\
24[23 ; 27]\end{array}$ & $\begin{array}{l}31[27 ; 34] \\
26[24 ; 33]\end{array}$ & $\begin{array}{l}31[23 ; 36] \\
31[27 ; 36]\end{array}$ & $\begin{array}{l}33[24 ; 35] \\
26[24 ; 37]\end{array}$ & \\
\hline $\begin{array}{l}\text { Lac-Clear. } \\
\mu \mathrm{mol} / \mathrm{min} / \mathrm{kg}\end{array}$ & $\begin{array}{l}\text { CNT } \\
\text { MEG }\end{array}$ & $\begin{array}{l}15[13 ; 23] \\
12[10 ; 16]\end{array}$ & $\begin{array}{c}3[-4 ; 6] \# \\
5[2 ; 9] \#\end{array}$ & $\begin{array}{c}-4[-8 ; 0,6] \# \\
4[-6 ; 10] \#\end{array}$ & $\begin{array}{l}-8[-12 ;-6] \# \\
0,4[-15 ; 7] \#\end{array}$ & \\
\hline $\begin{array}{l}\text { L/P ratio } \\
\text { hv }\end{array}$ & $\begin{array}{l}\text { CNT } \\
\text { MEG }\end{array}$ & $\begin{array}{l}15[13 ; 19] \\
14[12 ; 16]\end{array}$ & $\begin{array}{l}22[16 ; 30] \# \\
20[17 ; 22] \#\end{array}$ & $\begin{array}{l}29[20 ; 75] \# \\
22[19 ; 31] \#\end{array}$ & $\begin{array}{c}64[24 ; 100] \# \\
50[17 ; 94] \#\end{array}$ & \\
\hline
\end{tabular}

\section{P52 Endotoxin-induced pulmonary hyporesponsiveness to inhaled nitric oxide is improved by nitric oxide synthase 2 inhibition}

\section{Bopp, A Holzmann, H Schmidt, MM Gebhard* and E Martin}

Department of Anaesthesiology and *Department of Experimental Surgery, Ruprecht-Karls-University, INF 110, 69120

Heidelberg, Germany

Introduction: In isolated-perfused lungs of endotoxin-challenged rats, inhibition of nitric oxide synthase 2 (NOS2) by aminoguanidine (AG) improved responsiveness to inhaled nitric oxide (NO) [1]. In dogs treated with lipopolysaccharide (LPS), S-methylisothiourea (SMT) and AG prevented worsening of hemodynamics and gas exchange [2]. In this study, inhibition of NOS2 by SMT and AG modulates responsiveness to inhaled NO in lungs of endotoxin (LPS)challenged rats in a dose-dependent manner.

Methods: Sprague-Dawley rats (400-450 gm BW) were injected i.p. with $0.5 \mathrm{mg} / \mathrm{kg}$ E. coli 0111:B4 LPS ( $=42)$ or were controls $(n=9)$. Four hours later, LPS-treated rats were injected i.p. with 3, 10, and $30 \mathrm{mg} / \mathrm{kg} \mathrm{AG}$ or 0.01 , $0.1,1$ and $10 \mathrm{mg} / \mathrm{kg}$ SMT. $18 \mathrm{~h}$ later, lungs were isolatedperfused in situ using Hank's solution. Pulmonary artery pressure (PAP) was elevated by $6-8 \mathrm{mmHg}$ using the thromboxane analogue U46619. Decrease of PAP in response to inhaled $\mathrm{NO}(0.4,4$, and $40 \mathrm{ppm} \mathrm{NO}$ in random order) was measured. Serum and lung perfusate nitrate/nitrite levels were measured with the Griess-reaction.

Results: See Table.

Conclusions: $\mathrm{AG}$ is more effective in improving $\mathrm{NO}$ responsiveness than SMT. Responsiveness to inhaled NO appears to be dependent on the degree of NOS2 inhibition in lungs of LPS-challenged rats, because improve-

\begin{tabular}{lccc} 
& \multicolumn{3}{c}{$\Delta \mathrm{PAP}(\mathrm{mmHg})$} \\
\hline Control & $0.4 \mathrm{ppm} \mathrm{NO}$ & $4 \mathrm{ppm} \mathrm{NO}$ & $40 \mathrm{ppm} \mathrm{NO}$ \\
\hline LPS+3mg/kg AG & $-1.94 \pm 0.63$ & $-4.01 \pm 1.16$ & $-5.28 \pm 0.86$ \\
LPS+10mg/kg AG & $-1.98 \pm 1.12^{\star}$ & $-4.02 \pm 2.16^{\star}$ & $-6.22 \pm 1.65^{\star}$ \\
LPS+30mg/kg AG & $-1.32 \pm 0.79 \#$ & $-3.37 \pm 1.80^{\star}$ & $-5.53 \pm 1.27^{\star} \#$ \\
LPS+0.01mg/kg SMT & $-0.44 \pm 0.33 \#$ & $-1.60 \pm 1.23 \#$ & $-2.64 \pm 1.71$ \\
LPS+0.1 mg/kg SMT & $-0.24 \pm 0.27 \#$ & $-1.28 \pm 0.97 \#$ & $-1.96 \pm 1.51 \#$ \\
LPS+1 mg/kg SMT & $-1.75 \pm 1.32^{\star}$ & $-3.70 \pm 0.79^{\star}$ & $-5.75 \pm 2.47^{\star}$ \\
LPS+10mg/kg SMT & $-0.30 \pm 0.42 \#$ & $-0.50 \pm 0.51 \#$ & $-1.77 \pm 0.40 \#$ \\
LPS & $-0.12 \pm 0.15 \#$ & $-0.64 \pm 0.68^{\star}$ & $-0.98 \pm 0.65 \#$ \\
\hline
\end{tabular}

${ }^{*} P<0.05$ vs treatment with LPS alone; \# $P<0.05$ vs control; data are mean \pm SEM (Scheffe-test).

ment of NO responsiveness correlated with nitrate/nitrite levels in serum and lung perfusate.

\section{References:}

1. Holzmann A: Anaesthesiology 1999, 91(I):215-221.

2. Numata M.: J Immunol 1998, 160(6):3031-3037. 
P53 In contrast to CD64 expression, CD14 expression is persistently downregulated on polymorphonuclear cells (PMN) but not on monocytes of patients with septic shock

E Barth, J Wollmeyer, G Fischer, T Weckmann, M Appel, EM Schneider, LL Moldawer*, M Georgieff and M Weiss

Department of Anesthesiology, University of Ulm, Steinhoevelstr. 9, 89070 Ulm, Germany; *Department of Surgery, University of Florida, Gainesville, USA

Objectives: To investigate the time course of surface CD14 and CD64 on PMN and on monocytes, in postoperative/post-traumatic patients with septic shock, from the first day of septic shock onwards.

Methods: Over a six month period, in 22 patients admitted to the intensive care unit, a daily analysis of CD14 and CD64 surface expression on PMN and monocytes was performed by flow cytometry (Becton Dickinson, USA). All patients suffered from septic shock for three days at least. Box plots of the mean intensity (Mnl) values of surface CD14 and CD64 are given for days 1-14. Statistical analysis was performed by Wilcoxon rank sum test $(P<0.05$; significant intragroup differences between the day indicated by a star $\left.{ }^{*}\right]$ and the following days denoted by a line over the respective days [I-I]). In addition, normal range of controls $[\mathrm{C}]$ is presented by a box plot.
Results: CD64 is significantly upregulated on PMN and monocytes compared to healthy volunteers, whereas CD14 expression is significantly decreased on all days. Regarding the time course, a significant decrease in CD14 and CD64 expression on PMN occured within the first three days. In monocytes, compared with the first two days, CD64 expression was diminished from day 3 onwards, however, CD14 expression on day 4, only.

Conclusions: Upregulation of CD64 expression on PMN and monocytes indicates leukocyte activation in patients with septic shock. In contrast to CD64, surface CD14 on PMN decreased and is continuously downregulated over time, whereas no decrease in surface CD14 on monocytes is observed. This may be due to different membrane anchors and/or different LPS-CD14 signaling in PMN and monocytes in patients during septic shock.

Figure
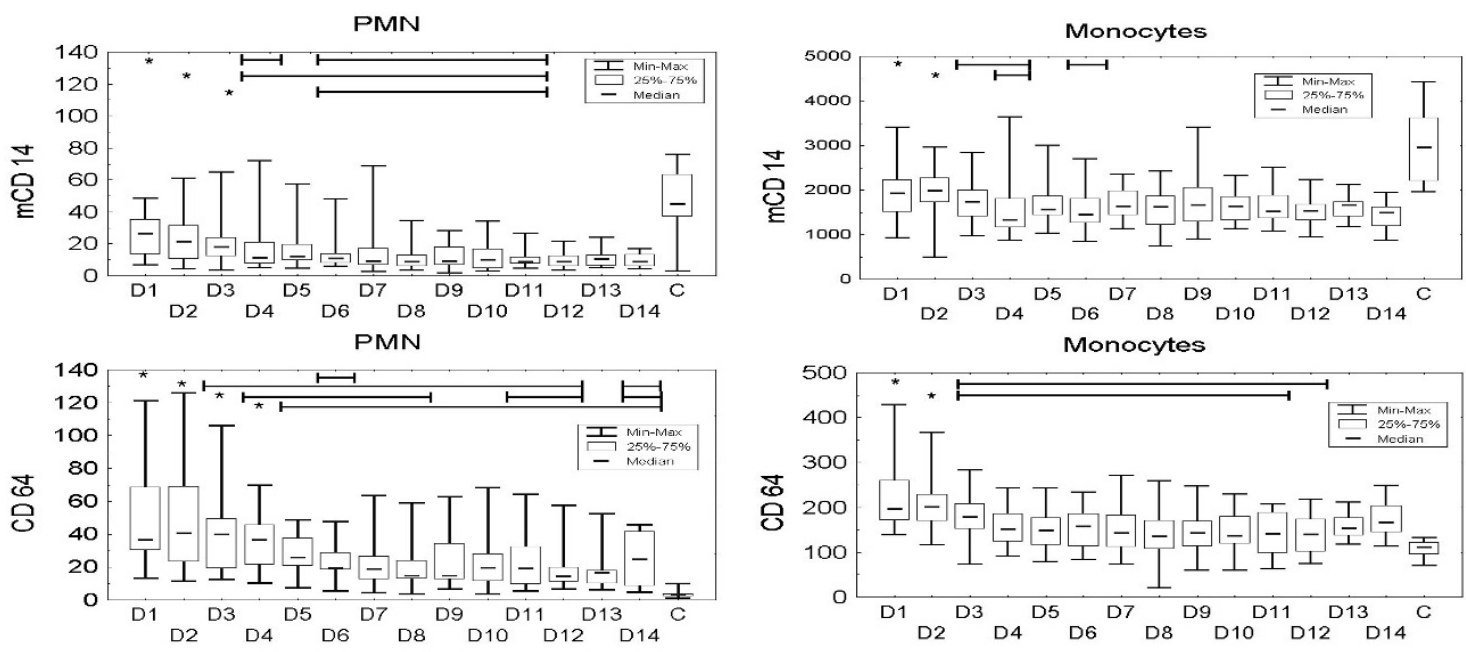

\section{Respiratory burst activity of PMN is increased in most patients during septic shock}

\section{E Barth, G Fischer, F Börner, LL Moldawer*, EM Schneider, M Georgieff and M Weiss}

Department of Anesthesiology, University of Ulm, Steinhoevelstr. 9, 89070 Ulm, Germany; *Department of Surgery, University of Florida, Gainesville, USA

Objectives: To investigate whether burst activity of polymorphonuclear cells (PMN) of postoperative/post-traumatic patients during septic shock is predominantly increased, unchanged or decreased compared to that of healthy volunteers.
Methods: Over a period of six months, 12 patients (four survivors and eight nonsurvivors) with septic shock for at least three days duration were studied during their period of septic shock on the intensive care unit. $E$. coli induced burst activity of granulocytes was determined by flow 
cytometry (Bursttest ${ }^{\mathrm{TM}}$, Orpegen, Heidelberg, Germany). The PMN burst activity of 12 healthy volunteers served as normal range.

Results: PMN burst activity in healthy volunteers ranged from 5-18 $\mathrm{Mnl}$ (median 9.2). Compared to this normal range, burst activity of patients with septic shock was predominantly (i.e. on more than $50 \%$ of days in septic shock) greater than that of healthy volunteers, in all patients, survivors and non-survivors.

Conclusions: PMN burst activity is predominantly increased in most patients with septic shock compared to healthy volunteers, indicating an activation of PMN during septic shock. Moreover, burst activity of granulocytes
Number of patients with more than $\mathbf{5 0 \%}$ of days during septic shock within a distinct range of PMN burst activity compared to the normal range

\begin{tabular}{lccc}
\hline Mnl & $\begin{array}{c}\text { below } \\
<5\end{array}$ & $\begin{array}{c}\text { within } \\
5-18\end{array}$ & $\begin{array}{c}\text { above } \\
>18\end{array}$ \\
\hline Survivors & $0 / 4$ & $2 / 4$ & $2 / 4$ \\
Non-survivors & $0 / 8$ & $2 / 8$ & $6 / 8$ \\
All patients & $0 / 12$ & $4 / 12$ & $8 / 12$ \\
\hline
\end{tabular}

during septic shock does not discriminate survivors from non-survivors.

P55 Differences in phagocytosis by polymorphonuclear leucocytes (PMNs) and monocytes (Mos) in endotoxemia and endotoxin (ET) tolerance

K-H Staubach, J Nolde, L Song, H Brade* and H-P Bruch

Department of Surgery, Medical University of Lübeck, Ratzeburger Allee 160, 23538 Lübeck, *Research Center Borstel, Medical University of Lübeck

Flow cytometry offers a rapid method to quantitate phagocytosis by PMNS and Mos. Since the PMN is a major defense beside the Mos against bacterial infection we compared both systems in a porcine endotoxin shock model in control animals (C) and endotoxin-tolerant animals ( $\mathrm{T}$ ). A commercial test kit (phagotest, Orpegen, Heidelberg, Germany) was used for demonstrating ingestion of opsonized fluorescent $E$. coli $(\mathrm{Fl})$ and phagocytosing cells (PC). Seven pigs were pretreated over five days with increasing doses of atoxic endotoxin from Rhodopseudomonas sphaeroides $(\mathrm{T})$ before induction of an ET shock with ET from Salmonella friedenau at a dose of $250 \mathrm{ng} / \mathrm{kg} \mathrm{BW/h}$, like the controls who were not pretreated.

Results of both groups were expressed in fluorescence intensity (FI) and summarized in the Table.

The functional assessment of Mos and PMNS in our shock model revealed an enhancement of phagocytic activity in endotoxin-tolerant animals both in Mos and PMNS with the highest increase in the latter.

\begin{tabular}{|c|c|c|c|c|c|c|c|c|c|c|c|}
\hline & & & Days (ir & nunizatic & period) & & & & rs (ET sl & & \\
\hline & $\mathrm{FI}$ & I & II & III & IV & V & 1 & 3 & 6 & 9 & 12 \\
\hline Mos & C & 679 & 715 & 631 & 670 & - & 459 & 723 & 554 & 210 & - \\
\hline Mos & $\mathrm{T}$ & 532 & 639 & 529 & 438 & - & 294 & 683 & 617 & 743 & 703 \\
\hline PMNS & C & 996 & 886 & 1007 & 940 & - & 1316 & 1810 & 1375 & 501 & - \\
\hline PMNS & $\mathrm{T}$ & 1165 & 1096 & 984 & 1082 & - & 555 & 1426 & 1343 & 1964 & 2146 \\
\hline & PC & I & II & III & IV & V & 1 & 3 & 6 & 9 & 12 \\
\hline Mos & C & 15.0 & 15.0 & 15.0 & 15.0 & - & 19.2 & 15.6 & 9.7 & 2.9 & - \\
\hline Mos & $\mathrm{T}$ & 10.6 & 16.7 & 14.9 & 5.9 & - & 20.8 & 23.6 & 19.3 & 15.3 & 14.0 \\
\hline PMNS & C & 8.2 & 8.2 & 8.2 & 8.2 & - & 5.7 & 6.8 & 6.7 & 8.9 & - \\
\hline PMNS & $\mathrm{T}$ & 4.9 & 8.4 & 8.2 & 2.6 & - & 14.6 & 14.0 & 7.5 & 9.1 & 11.0 \\
\hline
\end{tabular}




\section{P56 Role of platelet activating factor (PAF) on leukocyte-independent plasma extravasation and mast} cell activation during endotoxemia

A Walther, N Yilmaz, W Schmidt, A Secchi, MM Gebhard*, E Martin and H Schmidt

Department of Anesthesiology, and *Department of Experimental Surgery, Ruprecht-Karls-University, Im Neuenheimer Feld 110 , 69120 Heidelberg, Germany

Introduction: Independently from leukocyte adherence, endothelial factors and mast cell activation promote microvascular permeability [1]. The platelet activating factor (PAF) has been shown to play a significant role in endotoxin-induced leukocyte adherence [2]. The aim of our study was to investigate if there is also a role of PAF in mediating leukocyte-independent microvascular permeability changes and activation of mast cells during endotoxemia. Therefore, during endotoxemia, microvascular permeability and mast cell activation were determined after inhibition of the L-selectin mediated leukocyte-adherence by fucoidin and after inhibition of PAF effects by the the PAF receptor antagonist BN52021.

Methods: In male Wistar rats, microvascular permeability (MP), leukocyte adherence (LA) and mast cell activation (MCA) were determined in mesenteric postcapillary venules using intravital microscopy at baseline and at 60 and 120 min after the start of a continuous infusion of endotoxin (ETX; $2 \mathrm{mg} / \mathrm{kg} / \mathrm{hr}$, E. coli O26:B6) (group A, $n=10$ ). Leukocyte-endothelial interaction was blocked using fucoidin (25 mg/kg b.w.). In addition to the proce- dure in group A, group $B(n=10)$ received BN52021 (5 $\mathrm{mg} / \mathrm{kg}$ b.w.) after baseline measurements. Group C $(n=10$; control group) only received equivalent volumes of $\mathrm{NaCl} 0.9 \%$. Statistical analysis was performed using student's t-test. A $P$-value $<0.05$ was considered significant.

Results: In groups $A$ and $B$, fucoidin prevented LA, and so there were no significant differences in LA between these groups. In group $A, M P$ and MCA significantly increased starting at $60 \mathrm{~min}$ ( $P<0.05$ vs baseline). There was no significant increase in MP and MCA in groups $B$ and $C$. Differences between groups $A$ and $B$ in MP and MCA were significant at $120 \mathrm{~min}$.

Conclusions: The results of this study demonstrate that PAF plays a significant role in the initiation of endotoxininduced leukocyte-independent plasma extravasation and mast cell activation.

\author{
References: \\ 1. Kubes P: Am J Physiol 1996, 271:H2438. \\ 2. Schmidt H: J Surg Res 1996, 60:29.
}

P57 TNF- $\alpha$ : a possible mediator of remote tissue injury after viper envenomation

O Szold, AA Weinbroum, R Ben-Abraham, TE Englender, D Ovadia, S Marmor, M Sorkine, R Flaison and P Sorkine

General Intensive Care and the Departments of Oncology and Pathology, Tel Aviv Sourasky Medical Center and the Sackler

Faculty of Medicine, Tel Aviv University, Tel Aviv, Israel; and Unite des Venins, Institute Pasteur, Paris,Cedex 15, France

Introduction: TNF is a potent inflammatory promoting agent that can potentiate organ and tissue injury. A possible role of TNF in causing local tissue damage following snakebite was recently demonstrated in an intact rat model. We compared the systemic effects of TNF on the hearts and lungs of rats following an intramuscular injection of a sub-lethal dose of Vipera asis venom (500 $\mu \mathrm{g} / \mathrm{kg}$, experimental groups) to equivalent injections of saline (control group).

Results: Systemic TNF activity, heart rate and blood pressure as well as lung permeability and neutrophil sequestration were then evaluated in both groups. The venom caused a significant reduction in heart rate and arterial blood pressure, and the serum TNF levels peaked after two hours. These values remained unchanged in the control group. In contrast, lung microvasculature permeability and neutrophil sequestration were not significantly different between the experimental and control groups.

Conclusions: This study of the systemic and inflammatory effects of Vipera aspis venom showed that intramuscular injection of the poison results in systemic effects that are possibly mediated in part by TNF. These findings may have therapeutic implications in the treatment of patients with severe systemic manifestations after snakebites in terms of the possible benefits of blocking TNF activity. \\ Phagocytosis of granulocytes is decreased in most patients with severe sepsis or septic shock} G Fischer, E Barth, R Remmele, LL Moldawer*, EM Schneider, M Georgieff and M Weiss Department of Anesthesiology, University of Ulm, Germany, and *Department of Surgery, University of Florida, Gainesville, USA

Objectives: To investigate the phagocytotic activity of granulocytes during septic shock in postoperative/posttraumatic patients compared to that of healthy volunteers.
Methods: Over a six month period 19 patients with septic shock were monitored on a daily basis during their stay in the intensive care unit. Phagocytotic activity of granulocytes was determined by flow cytometry (Phagotest $^{\mathrm{TM}}$ 
Orpegen, Heidelberg, Germany). The normal range of phagocytosis (expressed as mean intensity, Mnl) was defined by a control group of 11 healthy volunteers.

Results: Phagocytosis of healthy volunteers was in the range of $34 \mathrm{Mnl}$ and $149 \mathrm{Mnl}$. All survivors and 7 of 10 nonsurvivors (i.e. $84 \%$ of all patients) had a lower phagocytotic activity on more than $50 \%$ of days in septic shock compared to that of healthy volunteers. No patient had a phagocytosis greater than $149 \mathrm{Mnl}$, the upper value of the normal range.

Conclusions: Phagocytosis is decreased in most patients during septic shock compared to healthy volunteers. Thus diminished phagocytotic activity may contribute to an impaired mechanism of bacterial elimination or a reduced
Number of patients with more than $\mathbf{5 0 \%}$ of days during septic shock within a distinct range of phagocytosis during septic shock compared to the normal range.

\begin{tabular}{lcccc}
$\begin{array}{l}\text { Phagocytosis (Mnl) } \\
\text { compared to normal } \\
\text { range }\end{array}$ & $\begin{array}{c}\text { Below } \\
(<34)\end{array}$ & $\begin{array}{c}\text { Within } \\
(34-149)\end{array}$ & $\begin{array}{c}\text { Above } \\
(>149)\end{array}$ & Total \\
\hline Survivors & 9 & 0 & 0 & 9 \\
Nonsurvivors & 7 & 3 & 0 & 10 \\
Total & 16 & 3 & 0 & 19 \\
\hline
\end{tabular}

resolution of infection in these patients. Moreover, phagocytotic activity of granulocytes does not discriminate survivors and nonsurvivors of septic shock.

P59 Triiodothyronine $\left(\mathrm{T}_{3}\right)$ ameliorates the cytokine storm in rats with sepsis

T Yokoe*, M Negishi*, Y lino* and Y Morishita ${ }^{\dagger}$

${ }^{*}$ Department of Emergency and Critical Care Medicine, ${ }^{\dagger}$ Second Department of Surgery, Gunma University School of Medicine, 3-39-15 Showa-machi Maebashi Maebashi, Gunma 371-8511, Japan

We reported that $T_{3}$ eliminated acidosis and improved survival rate in a rat model of sepsis. The aim of this study is to clarify the effect of $T_{3}$ on cytokine levels.

Male Sprague-Dawley rats, weighing 350-420 g, were ligated in the cecum with puncture (CLP method) under anesthesia with pentobarbital $(40 \mathrm{mg} / \mathrm{kg})$. After CLP, 50 $\mathrm{ml} / \mathrm{kg}$ of saline was injected subcutaneously for fluid resuscitation. Rats were assigned two groups; no treatment group (control group, $n=15$ ) and a $T_{3}$ treated group $(n=10)$. The $T_{3}$ treated group was given $3 \mathrm{ng} / \mathrm{hr}$ of $T_{3}$ using a osmotic pump embedded subcutaneously. Survival rate, levels of IL-1 $\beta$, TNF- $\alpha$, IL- 6, IL-8, and IL-10 were studied $24 \mathrm{~h}$ after surgery.
Three animals died in the control group, whereas no animals died in $T_{3}$ treated group. There was no significant difference on mean value of IL1- $\beta$ and TNF- $\alpha$ between two groups. Mean levels of IL- 6 and IL-8 in the control group were $237.4 \mathrm{pg} / \mathrm{ml}$ and $5342.7 \mathrm{pg} / \mathrm{ml}$, respectively, however, those in the $T_{3}$ group were suppressed to the level of $183.5 \mathrm{pg} / \mathrm{ml}$ and $55.2 \mathrm{pg} / \mathrm{ml}$, respectively $(P<0.05)$. Mean IL-10 level $(6.0 \mathrm{pg} / \mathrm{ml})$ in the $\mathrm{T}_{3}$ treated group was lower than that $(72.4 \mathrm{pg} / \mathrm{ml})$ in the control group $(P<0.05)$.

It is concluded that $T_{3}$ prevents the cytokine storm and improves the survival rate in this rat model of sepsis.

P60 Interleukin-17 stimulates intraperitoneal neutrophil infiltration through the release of the chemokine GRO $\alpha$ from peritoneal mesothelial cells

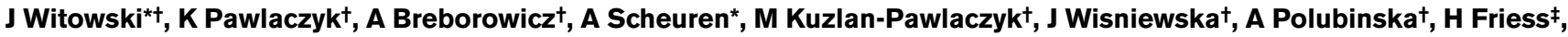
GM Gahl*, U Frei* and A Jörres*

*Nephrology and Medical Intensive Care, UK Charité, Campus Virchow-Klinikum, Augustenburger Platz 1, D-13353 Berlin,

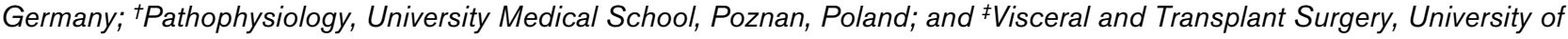
Bern, Inselspital, Bern, Switzerland

Introduction. IL-17 is a newly discovered cytokine implicated in the regulation of hematopoiesis and inflammation. Since IL-17 production is restricted to activated T lymphocytes the effects exerted by IL-17 may help to understand the contribution of $T$ cells to the inflammatory response. We investigated the role of IL-17 in leukocyte recruitment into the peritoneal cavity.

Methods: Leukocyte infiltration in vivo was assessed in BalB/ CJ mice. Effects of IL-17 on chemokine generation in vitro were examined in human peritoneal mesothelial cells (HPMC).
Results: Intraperitoneal administration of IL-17 resulted in a selective recruitment of neutrophils into the peritoneum and increased levels of $\mathrm{KC}$ chemokine (murine homologue of human GRO $\alpha$ ). Pre-treatment with anti-KC antibody significantly reduced the IL-17-driven neutrophil accumulation. Primary cultures of HPMC expressed IL-17 receptor mRNA. Exposure of HPMC to IL-17 led to a dose- and time-dependent induction of GRO $\alpha$ mRNA and protein. Combination of IL-17 together with TNF $\alpha$ resulted in an increased stability of GRO $\alpha$ mRNA and synergistic 
release of GRO $\alpha$ protein. Anti-IL-17 antibody blocked the effects of IL-17 in vitro and in vivo.
Conclusions: IL-17 is capable of selectively recruiting neutrophils into the peritoneal cavity via the release of neutrophilspecific chemokines from the peritoneal mesothelium.

P61 The ex vivo production of tumor necrosis factor- $\alpha$ (TNF- $\alpha$ ) in men: relationship to cytokine balance during cardiopulmonary bypass

\author{
Y Tabardel', L Dumont ${ }^{\dagger}$, D Schmartz ${ }^{*}$ JL Vincent ${ }^{\ddagger}$ and J Duchateau \\ Departments of *Anesthesiology and ${ }^{\ddagger}$ Intensive Care, Erasme University Hospital, Departments of ${ }^{\dagger}$ Anesthesiology and \\ \#Immunology, Brugmann University Hospital, Free University of Brussels, Brussels, Belgium
}

Objectives: To discriminate patients with strong or weak ex vivo TNF- $\alpha$ synthesis in response to whole blood stimulation with endotoxin (LPS) and to relate this ex vivo response to cytokine balance during and after cardiac surgery with cardiopulmonary bypass (CPB).

Patients and methods: 19 patients scheduled for coronary artery bypass graft (CABG) with CPB and 29 adult volunteers were entered into the study. Whole blood was stimulated with LPS, incubated for $16 \mathrm{~h}$ and TNF- $\alpha$ concentrations determined in the culture supernatant. Results obtained in both patients and volunteers were pooled. Individuals showing TNF- $\alpha$ concentrations lower or higher than the median value of $1052 \mathrm{pg} / \mathrm{ml}$ were defined as low and high responders, respectively. In the 19 patients, cytokine balance during and after CPB was evaluated by the plasma concentrations of TNF- $\alpha$, interleukin (IL)-8 and IL-10.

Results: In patients undergoing cardiac surgery, perioperative cytokine plasma levels were not significantly different in both low and high responders. In these two groups $e x$ vivo TNF- $\alpha$ production did not correlate with either TNF- $\alpha$ or IL-8 plasma levels measured during and after CPB. In contrast, ex vivo TNF- $\alpha$ production inversely correlated with IL-10 plasma concentrations observed four hours after the end of CPB in the low responder group (Spearman, $-0.76, P=0.01$ ) and tended to do so in the high responder group (Spearman, $-0.62, P=0.1$ ).

Conclusion: In our series, the ex vivo production of TNF$\alpha$ does not allow us to predict the magnitude of the systemic inflammatory response related to cardiac surgery, as evaluated by the plasma levels of TNF- $\alpha$ and IL8 during and after CPB. The negative correlation observed between the ex vivo TNF- $\alpha$ production and the production of IL-10 after CPB suggests that patients with a low ex vivo capability to synthesize the pro-inflammatory TNF- $\alpha$ have a high potential to synthesize the anti-inflammatory cytokine IL-10 in vivo. Whether this profile can be considered a risk factor for morbidity after cardiac surgery, as it has been shown for infectious diseases remains to be assessed. \\ Relationship between TNF $\alpha$ and IL10 release during cardiac operations and the development of apoptosis
}

M Qing*, J Vazquez-Jimenez†, B Klosterhalfen, K Schumacher*, A Pütz-Ebert*, J Duchateau§, G von Bernuth* and M-C Seghaye*

${ }^{*}$ Department of Pediatric Cardiology, ${ }^{+}$Cardiac Surgery, ${ }^{\ddagger}$ Pathology, Aachen University of Technology, Pauwelsstrasse 30 , D-52057, Aachen, Germany; §Department of Immunology, Hopital Brugman, Brussels, Belgium

Aim: To analyze the role of tumor necrosis factor (TNF) $\alpha$ and interleukin (IL) 10 produced during cardiac operations on the development of apoptosis in vital organs and the effect of core temperature during CPB on these finding.

Methods: 16 young pigs were assigned to a temperature $\left(\mathrm{T}^{\circ}\right)$ regimen during standardized CPB: normothermia ( $\mathrm{T}^{\circ}$ $\left.37^{\circ} \mathrm{C} ; n=8\right)$ and moderate hypothermia $\left(T^{\circ} 28^{\circ} \mathrm{C} ; n=8\right)$. TNF $\alpha$ and IL10 were determined by a pig specific ELISA. Six hours post-operative, tissue probes of the heart, liver, lung, and kidney were taken for standard- and immuno-histological examinations. Apoptotic cells were detected by an in situ apoptosis detection kit (TUNEL). Necrotic cells were counted by light microscopy.

Results: Pigs operated on in normothermia showed significantly higher TNF $\alpha$ production and lower IL10 production during and after CPB, and higher degree of cellular necrosis but lower degree of cellular apoptosis than pigs operated on in moderate hypothermia. In all animals, postoperative IL10 levels were negatively correlated with the production of myocardial apoptosis $(P=0.005)$ and $\mathrm{TNF} \alpha$ levels correlated significantly with necrosis in the heart and liver $(P<0.05)$.

Conclusion: Cardiac operations are related to cell death via both the necrotic and the apoptotic pathways. In this setting, IL10 could be an inductor of apoptosis. By increasing the production of IL10 and the ratio apoptosis/necrosis, moderate hypothermia during CPB might therefore provide organ protection. 


\section{P63 Elevated levels of adhesion molecules in septic patients with pre-existing coronary artery disease}

\section{R Wittich*, H Kern, WJ Kox, G Haring* and C Spies}

Department of Anesthesiology and Intensive Care, University Hospital Charité, Humboldt-University, D-10098 Berlin, Germany; *Department of Anesthesiology and Intensive Care, Carl-Thiem-Hospital, Thiemstrasse 111, D-03048 Cottbus, Germany

Introduction: Sepsis, as well as coronary artery disease $(C A D)$, is associated with high levels of circulating adhesion molecules [1,2]. Elevated plasma levels of these molecules may have clinical significance as markers of endothelial injury [2]. The purpose of the study was to investigate whether CAD influences the liberation of plasma adhesion molecules, cytokines and markers of the NO-system in septic patients and if these parameters may serve for identification of patients at greater risk for cardiovascular complications in sepsis.

Methods: After approval by the local ethical committee and obtaining informed consent from relatives, 44 septic patients were included in this study, of whom 24 patients met the criteria of CAD [3]. Measurements were performed within $12 \mathrm{~h}$ (early sepsis) of onset of sepsis and after $72 \mathrm{~h}$ (late sepsis). Soluble adhesion molecules sEselectin, and soluble intercellular adhesion molecule (sICAM)-1 were analyzed by commercially available ELISA. NO-markers were measured by the Griess reaction and cyclic guanosine monophosphate (cGMP) by competitive radioimmunoassay.

Results: Basic patient characteristics did not differ between groups. CAD patients showed significantly increased sICAM-1 levels in early and late sepsis (Table 1) but this failed to be predictive for cardiovascular complications. ICAM-1 and cGMP correlated positively in both groups with the intravenously administered norepinephrine
Table 1

\begin{tabular}{llcc}
\hline & & CAD $n=24$ & non-CAD n=20 \\
\hline \multirow{2}{*}{ sE-selectin $(\mathrm{ng} / \mathrm{ml})$} & Early & $135(33-352)$ & $141(56-320)$ \\
& Late & $98(34-472)$ & $49(25-175)^{\star}$ \\
\multirow{2}{*}{ sICAM-1 $(\mathrm{ng} / \mathrm{ml})$} & Early & $614(207-1616)$ & $446(207-867)^{\star}$ \\
& Late & $536(203-1479)$ & $351(202-764)^{\star}$ \\
cGMP $(\mathrm{mmol} / \mathrm{l})$ & Early & $17(6-46)$ & $13(6-44)$ \\
& Late & $35(8-51)$ & $17(3-52)^{\star}$ \\
\hline
\end{tabular}

median and range, ${ }^{*} P<0.05$ between groups.

dosage in early and late sepsis. Cardiac index, oxygen delivery and mixed venous oxygen saturation were significantly decreased in CAD patients in early and late sepsis $(P<0.05)$, whereas $\mathrm{VO}_{2}$ did not differ significantly between both groups. Mortality was increased in tendency in CAD patients ( $75 \%$ vs $50 \%$ in non-CAD).

Conclusion: Plasma levels of sICAM-1, sE-selectin and cGMP were elevated in CAD but may not serve as markers for cardiovascular complications in sepsis. The tendency in increased mortality rate could be due to an impaired endothelial reserve in CAD patients.

\footnotetext{
References:

1. Spies C et al:: Chest 1998; 113:1055-1063.

2. Hwang SJ et al.: Circulation 1997; 96: 4219-4225.

3. Mangano D: Anesthesiology 1990; 72:153-184.
}

\section{P64 Are IL-6, IL-10 and PCT plasma concentrations more reliable than APACHE-III or SAPS-II for the} individual mortality risk prediction in severe sepsis?

\section{Müller, G Drüge, $\mathbf{O}$ Eichelbrönner and N Roewer}

Bayerische Julius-Maximilians-Universität Würzburg, Klinik für Anaesthesiologie, Josef-Schneider-Strasse 2, D-97070 Würzburg, Germany

Introduction: Scoring systems such as APACHE-III and SAPS-II often fail to reliably predict the individual mortality risk particularly in patients with severe sepsis. Cytokines and procalcitonin (PCT) have been shown to play a crucial role both in the pathogenesis of sepsis and as diagnostic tools for the inflammatory process. The objective of this study was to investigate the relationship between plasma cytokine and PCT concentrations and the actual and predicted mortality using APACHE-III and SAPS-II in patients with severe sepsis.

Material and methods: The SCCM/ACCP selection criteria were used to identify the patients with severe sepsis. After severe sepsis was diagnosed SAPS-II and APACHE-III scores were calculated. In addition, blood samples were taken daily for the analysis of IL-6, IL-10 and PCT plasma concentrations for three consecutive days. IL6 and IL-10 were analysed using a sandwich type of a immunoenzymatic assay (Immunotech, Marseille, France) PCT plasma concentrations were measured using a specific, ultra sensitive immunoluminometric assay (LUMltest PCT assay, BRAHMS Diagnostica, Berlin, Germany).

Results: Out of the 29 patients of this study, 13 patients died leading to a mortality rate of $44.8 \%$. The APACHE-III and SAPS-II scoring systems produced almost identical results for the predicted mortality risks, but the areas under the ROC-curves (AUC) formed by the plot of possible pairs of false-positive and true-positive rates exhibited a poor prognostic validity for both scores (day 1: 0.5612; 
day 2: 0.5593; day 3: 0.5732). The plasma PCT concentrations were significantly higher in non-survivors compared to survivors (day 1: $P<0.01$; day 2: $P<0.07$; day 3 : $P<0.015)$. However, a discrimination value, to simply distinguish between survivors and non-survivors, was not found. The determination of plasma IL-6 concentrations on the first three days after inclusion into the study showed no significant difference between survivors and non-survivors. The IL-10 plasma concentrations of the patients who died were significantly higher compared with those of the survivors measured on the first two days (day 1: $P<0.001$; day 2: $P<0.005)$. The degree of correlation between PCT-values and the APACHE-III scores on all days of study was high (day 1: $P<0.0004$; day 2: $P<0.001$; day 3: $P<0.03$ ). For the PCT plasma concentrations and the SAPS-II score, a good correlation was only found on the first day (day 1: $P<0.005$ ). The IL-6 concentrations showed a good correlation with the APACHE-III scores only on the first day (day $1: P<0.04$ ) and the IL-10 concentrations correlated well with the APACHE-III score at day 1 and day 3 (day 1: $P<0.006$; day $3: P<0.026$ ). No correlation was found between the interleukin concentrations and the SAPS-II scoring system.

Conclusion: Our results demonstrate that the APACHEIII and the SAPS-II scoring systems evaluate a similar mortality rate in severe sepsis. However, in terms of individual mortality risk prediction, the reliability and precision of both scores is limited and does not allow a precise statement about the mortality risk. In contrast, the plasma concentrations of PCT and IL- 10 were significantly higher in the non-survivors and showed a good correlation with the actual mortality. IL-6 was not useful in this context. Thus, daily analyses of plasma PCT and IL-10 concentrations might be a helpful tool to improve the individual mortality risk prediction in patients with severe sepsis, possibly in combination with scores assessing the severity of illness.

\section{Tumor necrosis factor receptor I, II and tumor necrosis factor- $\alpha$ levels in patients with burns}

\section{S Endo*, Y Yamada*, $\mathrm{K}$ Inada $^{\dagger}$ and $S$ Sato ${ }^{\dagger}$}

Critical Care and Emergency Center, Department of Bacteriology, School of Medicine, Iwate Medical University, 19-1 Uchimaru, Morioka 020-8505, Japan

Objects: The present study was undertaken to examine the relationship between the severity of burns and TNFRI and TNFRII levels in the acute post-burn period and the entire follow-up period.

Materials and methods: Tumor necrosis factor $\alpha$ (TNF- $\alpha$ ) and TNF receptor I and II (TNFRI and TNFRII) were studied in 24 burn patients who had a total burn surface area (TBSA) of $50.2 \pm 20.4 \%$. TNFRI and TNFRII were quantified by enzyme-linked immunosorbent assay (ELISA) using a kit (Amersham, Buckinghamshire, UK). TNF- $\alpha$ was quantified by ELISA (Medogenix, Fleurus, Belgium).

Results: Immediately after the injury, both the TNFRI and TNFRII levels correlated significantly with TBSA $(r=0.7344, P<0.0001 ; r=0.6074, P=0.0012)$. The TNFRI and TNFRII levels immediately after the injury were significantly higher in the 11 patients who later died of their burns than in the 13 patients who survived $(0.8 \pm 0.4 \mathrm{ng} / \mathrm{ml}$ vs $1.8 \pm 0.7 \mathrm{ng} / \mathrm{ml}, P=0.0002 ; 2.3 \pm 1.1 \mathrm{ng} / \mathrm{ml}$ vs $4.5 \pm 1.6$ $\mathrm{ng} / \mathrm{ml}, P=0.0009$ ). The TNF- $\alpha$ levels immediately after the injury did not differ significantly between the group that survived and the group that died. The TNFRI and TNFRII values for the entire follow-up period also correlated significantly with TBSA. Peak TNFRI and TNFRII levels were significantly higher in the group that died than in the group that survived $(6.0 \pm 4.7 \mathrm{ng} / \mathrm{ml}$ vs $14.1 \pm 7.8 \mathrm{ng} / \mathrm{ml}$, $P=0.0009 ; 7.0 \pm 5.1 \mathrm{ng} / \mathrm{ml}$ vs $16.7 \pm 5.2 \mathrm{ng} / \mathrm{ml}, P=0.0003$ ).

Conclusion: The TNF- $\alpha$ levels correlated significantly with both the TNFRI and the TNFRII levels. The TNFRI and TNFRII levels thus closely reflected the severity of the burns in both the acute post-burn period and the subsequent follow-up period. In other words, these parameters well reflected the severity and outcome of the burns, irrespective of the presence or absence of accompanying infection.

P66 Cytokines and sepsis - just black smoke?

S Marum, JP Ribeiro, E Arranhado*, H Lage*, L Mota, P Marcelino, AP Fernandes, J Oliveira and MR Silva

Hospital de Curry Cabral, Unidade de Cuidados Intensivos, *Laboratório de Imunologia/Nefrologia, Rua da Beneficiência, $n^{\circ} 8$, 1069-166, Lisbon, Portugal

Introduction: Sepsis is a clinical syndrome resulting from an inflammatory response of the host to infection, mediated by the production of cytokines in circulation. The need to understand better the mechanisms that lead to multiple organ failure, the authors (AA) began a study of cytokines in these patients.
Material and methods: In 1998 the AA began a prospective study of soluble cytokines, consisting at the moment of 54 patients, 36 with criteria of Sepsis/MODS (Multiple organ dysfunction syndrome) and 18 with SIRS (Systemic inflammatory response syndrome). The soluble cytokines (TNF $\alpha$, IL-6, IL-8 and IL-10) were measured in serum 
samples by the quantitative sandwich enzyme immunoassay (solid phase) - RD systems, Inc. Minneapolis (CITOMED, PORTUGAL) - with a previously established protocol.

Results: In the sepsis/MODS group, 7 patients with IL-6 $<300 \mathrm{pg} / \mathrm{ml}$ survived; among the ones with $\mathrm{IL}-6>300$ $\mathrm{pg} / \mathrm{ml}$, only the five patients submitted to pulses of methylprednisolone and/or emergent surgery survived. The patients with SIRS always presented low concentrations of cytokines, and 14 of them survived.
Conclusion: In the patients with Sepsis/MODS, the clinical severity was always related to high serum levels of IL6 , and/or its increase. Serum values of IL-6>300 pg/ml were predictive of death. Although TNF $\alpha$ and IL- 8 values were high in some patients, these showed a non-uniform behaviour, and the correlation of these values with clinical severity or outcome was not statistically significant as was IL-6. In those patients who survived, the clinical improvement was always followed by a remarkable decline in the serum values of IL-6. IL-6 is an important severity serum marker in equal or superior value to others described and accepted world-wide for patients with sepsis.

P67 The GH/IGF-I axis in children with a meningococcal septic shock: striking differences between survivors and non-survivors

F de Groof, ED de Kleijn, JA Hazelzet, P Uitterlinden, J v Doorn, ACS Hokken-Koelega and KFM Joosten

Department of Paediatrics, Sophia Children's Hospital, Dr Molewaterplein 60, 3015 GJ Rotterdam, The Netherlands

Introduction: Meningococcal septic shock is a life-threatening clinical syndrome that may lead to pronounced neuro-endocrine and metabolic alterations. In critically ill adults a $\mathrm{GH}$ resistant state has been well documented. Less information is available in critically ill paediatric patients. In the present study we evaluated the time course and pulsatile pattern of the $\mathrm{GH}$ secretion and the levels of serum IGF-I, IGFBP-1 and IGFBP-3.

Methods: Children with a septic shock and petechiae/purpura requiring intensive care treatment were enrolled in this study. The paediatric risk of mortality (PRISM) score was used to monitor the severity of disease. A GH profile was done in the first $6 \mathrm{~h}$ after admission and at day 3. The serum IGF-I, IGFBP-1 and IGFBP-3 levels were measured on admission $(T=0)$, and 24 and 48 $h$ after admission. The serum levels of IGF-I and IGFBP-3 were corrected for sex and age, expressed as SDS-levels.

Results: Twelve patients fulfilled the inclusion criteria, 7 boys and 5 girls, with a median age of 22 months. The median PRISM score was 23. Three of the 12 patients died. The serum levels of IGF-I and IGFBP-3 were decreased in all patients, the median SDS being respec- tively -2.6 and -5.7 . There was a significant difference between survivors and non-survivors regarding median $\mathrm{GH}$ levels during the first $6 \mathrm{~h}(7 \mathrm{mU} / \mathrm{l}$ vs $131 \mathrm{mU} / \mathrm{l}, P=0.01)$, serum IGF-I levels at $\mathrm{T}=0$ (43 $\mathrm{ng} / \mathrm{ml}$ vs $7 \mathrm{ng} / \mathrm{ml}, P=0.03)$, IGFBP-3 SDS levels at $\mathrm{T}=0$ ( -5.6 vs $-7.7, P=0.05)$, and PRISM score (18 vs $34, P=0.01$ ). The levels of serum IGFBP-1 at $\mathrm{T}=0$ were increased in the non-survivors in comparison with the survivors (median being respectively 1315 and $262 \mathrm{ng} / \mathrm{ml}$ ), but this difference didn't reach significance $(P=0.15)$. Non-survivors showed very high $\mathrm{GH}$ levels without $\mathrm{GH}$ peaks during the first $6 \mathrm{~h}$ after admission. In survivors, the $\mathrm{GH}$ secretion pattern at day 1 showed a larger number of $\mathrm{GH}$ peaks and larger $\mathrm{GH}$ peak amplitudes than at day 1. A significant increase in serum IGF-I levels was found in all patients between $T=0$ and $\mathrm{T}=24(P=0.04)$, followed by a significant decrease in serum IGF-I levels between $\mathrm{T}=24$ and $\mathrm{T}=48(P=0.01)$.

Conclusion: Mortality in children with a meningococcal septic shock is associated with extremely increased serum $\mathrm{GH}$ levels, no oscillatory $\mathrm{GH}$ secretion, high levels of IGFBP-1 and a high PRISM score. In those who survived there are significant changes during the first $48 \mathrm{~h}$ in the $\mathrm{GH} / \mathrm{IGF}-\mathrm{I}$ axis, suggesting a decrease in the $\mathrm{GH}$ resistance.

P68 Secretory phospholipase A2 (SPLA2), procalcitonin (PCT) and C-reactive protein (CRP) for the diagnosis and differentiation of septic shock and non-septic shock

O Anglès, MH Chabannier, E Bauvin, J Fauvel, Y Coulais, B Cathala and M Génestal

Service de Réanimation Adultes, Hôpital Purpan, F31059 Toulouse, France

Introduction: Serum SPLA2 and CRP levels increase in patients with systemic inflammatory response syndrome (SIRS). High serum levels of PCT have been detected in patients with inflammatory conditions from invasive bacterial and fungal infections. The aim of this study was to determine the diagnostic value of SPLA2, CRP and PCT in septic shock.
Patients and methods: Consecutive patients admitted to the ICU with shock were included. Patients were screened in two groups: septic shock (American college of chest physicians criteria) and non-septic shock. Upon admission, serum sPLA2, CRP and PCT were simultaneously analysed. Data, expressed as means $\pm S D$, were analysed by an independent investigator not involved in 


\begin{tabular}{lccc} 
& sPLA2 & CRP & PCT \\
\hline Septic & $153 \pm 202$ & $201 \pm 124$ & $47 \pm 83$ \\
Non-septic & $13 \pm 14$ & $83 \pm 116$ & $5.8 \pm 7,7$ \\
$P$ & $<0.0001$ & 0.0004 & 0.001 \\
\hline
\end{tabular}

Mann-Whitney test

ICU. The catalytic activity of SPLA2 was detected by fluorimetric assays (normal $10 \mathrm{mU} / \mathrm{ml}$ ). PCT was analysed by commercially available Lumitest ${ }^{\circledR}$ kit (BRAHMS, Berlin).

Results: Fifty-nine patients were included (20 women, 39 men), thirty-nine with septic shock (13 women, 26 men, mean age $=56 \pm 18)$ and twenty with non-septic shock (7 women, 13 men, mean age $=51 \pm 22$ ). Total mortality $=40 \%$. Septic shock mortality $=51 \%$ and non-septic shock mortality $=20 \%$.

sPLA2, CRP and PCT values were significantly higher in patients with septic shock. The areas under the curve (ROC) of SPLA2, CRP and PCT were respectively 0.896, 0.792 and 0.765 . The area under the curve of sPLA2 was significantly higher than PCT area $(P<0.05)$.
Figure

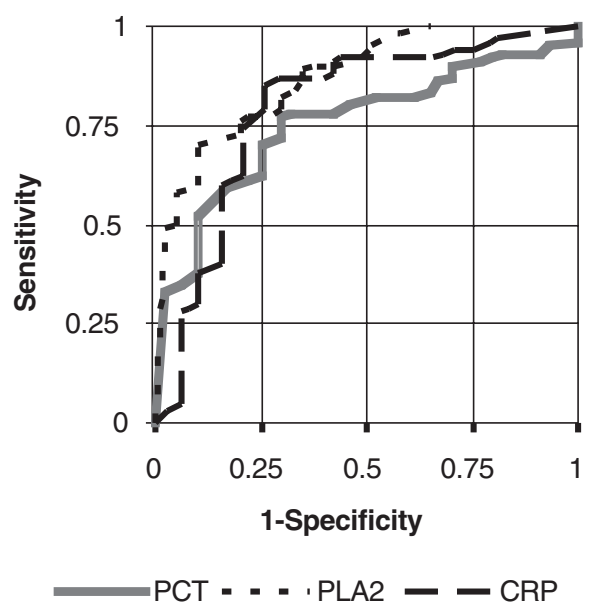

Conclusion: PCT does not appear to be a better marker to discriminate septic shock and non-septic shock than sPLA2 and CRP.

\section{P69 How does major abdominal surgery induce procalcitonin and IL-6 in the postoperative period?}

\section{EK Karpel}

Department of Anaesthesiology and Intensive Therapy, Silesian University of Medicine, ul. Medyków 14, 40-752 Katowice, Poland

Introduction: Major abdominal surgery is often complicated by systemic inflammatory response (SIRS), local or general infection, sepsis and even septic shock. It is important to evaluate the risk of these serious complications to prevent them. It has been demonstrated that circulating markers of inflammation can be helpful in early diagnosis of surgical infection in the postoperative period. The aim of my study was to evaluate how major abdominal surgery induces interleukin 6 and procalcitonin by itself and to determine the usefulness of these markers in diagnosis of infectious complications.

Study design: Prospective clinical study approved by local Ethics Committee.

Patients: Thirty patients, ASA II-IV, undergoing elective surgery of the gastrointestinal system were studied. Surgical procedures included: gastrectomy or oesophagus resection $(\mathrm{N}=10)$, Whipple's operation $(\mathrm{N}=10)$, bowel resection $(\mathrm{N}=5)$, partial liver resection $(\mathrm{N}=2)$, others $(\mathrm{N}=3)$. In all patients, antibiotic prophylaxis $(24-48 \mathrm{~h})$ was applied. Eleven patients developed local infection (wound $\mathrm{N}=6$, lung infection $\mathrm{N}=3$ ) or general infection $(\mathrm{N}=2)$. None of the analysed patients died; the total period of hospital treatment varied from 15 to 110 days.
Methods: Blood samples were taken during induction of anaesthesia and on days 1, 3, 5 and 7 after surgery. IL-6 measurements were performed by immunoradiometric assay (IL-6-IRMA, BIOSOURCE) and procalcitonin was measured by illuminometric method (PCT LUMltest, Brahms). Number of SIRS criteria and SOFA score were assessed at the same time points.

Results: We observed low plasma levels of both IL-6 and PCT prior to surgery and a slight increase in PCT at the first postoperative day in patients with signs of infection accompanied by increases in SIRS and SOFA assessment.

In two patients with general infection, the highest levels of PCT were 8.41 and 7.95 with IL-6 levels of 3500 and 3700 respectively.

\begin{tabular}{lcccccc} 
& \multicolumn{3}{c}{ Infection $(\mathrm{N}=11)$} & \multicolumn{3}{c}{ no infection $(\mathrm{N}=19)$} \\
\cline { 2 - 7 } $\begin{array}{l}\text { Time of } \\
\text { sampling }\end{array}$ & SIRS & PCT & IL-6 & SIRS & PCT & IL-6 \\
\hline Before surgery & 0 & 0.24 & 28.9 & 0.2 & 0.41 & 107 \\
1 day & 3.4 & 4.73 & 552 & 3.4 & 0.44 & 365 \\
3 day & 3.1 & 0.75 & 106 & 2.3 & 0.53 & 181 \\
5 day & 2.5 & 0.21 & 106 & 1.3 & 0.24 & 111 \\
7 day & 1.6 & 0.16 & 105 & 0.6 & 0.12 & 111 \\
\hline
\end{tabular}


Conclusions: Major abdominal surgery slightly increases procalcitonin serum levels in patients with infectious complications only, with the peak level 24-48 h after operation. Increase of IL-6 serum levels occurs $24-48 \mathrm{~h}$ after surgery in all patients and correlates with the assessment of inflammation measured by the number of SIRS criteria. PCT is a marker of infection rather than inflammation.

\section{P70 Role of decisive markers in diagnosis and outcome of patients with septic shock}

A Rizk, F Khalil, F Ragab, L Hamed and S Mokhtar

Critical Care Medicine Department, Cairo University Hospital, Cairo, Egypt

Introduction: Of the few biochemical parameters available to monitor critically ill patients and to control the course of therapy in severe inflammation, procalcitonin (PCT) and polymorphonuclear (PMN) elastase enzyme stand as innovative diagnostic parameters with features different from other presently routine indicators of the inflammatory response.

Methods: Our work aims at assessing the potential role of PCT and PMN elastase enzyme in early diagnosis and early prediction of prognosis in patients (pts) with sepsis and septic shock. To achieve this goal we studied 20 pts with septic shock ( 16 male and 4 female, mean age 50.15 years), together with a second group comprising 10 pts (9 male, mean age 49.2 years) with systemic sepsis without shock. A third group including 20 healthy volunteers matching with age and sex, served as controls. Serum PCT and PMN elastase enzyme levels were estimated on admission for both pts and control groups with other laboratory investigations and clinical parameters. A multivariate, discriminate analysis was performed using the following variables: PCT, PMN elastase enzyme, albumin, $\alpha$-1-antitrypsin, $\alpha$-2-macroglobulin, and C-reactive protein (CRP), as independent parameters.

Results: Comparing the three groups (septic shock, sepsis, and healthy control subjects) all together our results exhibited significantly higher PCT levels $(59.7 \pm 14$, $97.2 \pm 14$ vs $0.4 \pm 0.1 \mathrm{ng} / \mathrm{ml}, P<0.0000$ ), higher PMN elastase enzyme (183.4 $\pm 101,132.1 \pm 117$ vs $6.5 \pm 3 \mu \mathrm{g} / \mathrm{l}$, $P<0.0000)$, lower serum albumin $(2.1 \pm 0.6,2.6 \pm 0.9$ vs $4.7 \pm 0.5 \mathrm{gm} \%, P<0.05)$, higher $\alpha$-1-antitrypsin $(3.9 \pm 1$, $6.2 \pm 3$ vs $3.6 \pm 0.6 \mathrm{mg} / \mathrm{L}, P<0.0001)$, higher $\alpha$-2-macro- globulin (2.27 $\pm 0.8,2.4 \pm 1$ vs $0.7 \pm 0.2 \mathrm{mg} / \mathrm{l}, P<0.0000)$, higher CRP $(52.2 \pm 36, \quad 46.8 \pm 31$ vs $5.4 \pm 0.4 \mathrm{mg} / \mathrm{l}$, $P<0.0000)$.

With further patient subdivision to 6 survivors and 24 nonsurvivors only PCT could be identified as independent predictor for short term prognosis in patients with sepsis with an overall predictive accuracy $80 \%$ and with cut off value of $\geq 78 \mathrm{ng} / \mathrm{ml}$. While PMN elastase enzyme could be used as a parameter inside a model including the whole previous parameters with overall predictive accuracy $76.7 \%$, and with cut off value of $\geq 142 \mu \mathrm{g} / \mathrm{l}$.

Conclusion: Serum PCT and PMN elastase enzyme are independent useful diagnostic markers for early detection of systemic inflammatory response syndrome with or without shock. However PCT has the advantage over the above mentioned parameters in being significantly predictive of short-term prognosis, with overall predictive accuracy of $80 \%$. Procalcitonin, PMN elastase enzyme, $\alpha$-1-antitrypsin, $\alpha$-2-macroglobulin, CRP, and albumin, is a model which could be used for early prediction of complications of sepsis patients with overall predictive accuracy of $76.7 \%$.

\section{References:}

1. Gramm HJ et al: Procalcitonin - ein neuer marker der inflammatorischen wirtsantwort, longitudinalstudien bei patienten mit sepsis und peritonitis. Chir Gastroenterol 1995, 11(suppl.):51-54.

2. Bohuon $\mathrm{C}$ et al: Blood procalcitonin is a new biological marker of the human septic response. New data on the specificity. Supp/ Clin Intens Care 1994, 5:2

3. Enscnauer $\mathrm{M}$, Quintel $\mathrm{R}$, Kattermann J Aufenanger. Clin Investig 1994, 72:843-849.

4. Gardinali M et al:: Arch Surg 1992, 127(10):1219-1224.

\section{P71 A new diagnostic and severity marker in patients with SIRS, sepsis, severe sepsis and septic} shock; procalcitonin

S Çelebi, P Ergin Özcan, $\mathbf{S}$ Tugrul, $\mathbf{N}$ Çakar and F Esen

Department of Anesthesiology and Intensive Care, University of Istanbul, Medical Faculty, Istanbul, Turkey

Although the exact origin of procalcitonin (ProCT) in infection remains unclear, its association with the presence and severity of infection has been demonstrated. This prospective study aims to evaluate ProCT as a diagnostic marker of infection in critically ill patients with SIRS, and to investigate the relationship of ProCT levels with sepsis severity and outcome.
The study included 85 consecutive adult patients, fulfilling the criteria of inflammatory response, admitted to the ICU at the Istanbul University Hospital. The clinical state of the patients was defined on the basis of clinical, laboratory and bacteriological findings as SIRS, sepsis, severe sepsis and septic shock according to the consensus criteria. Data were collected including temperature, heart rate, 
Table 1

\begin{tabular}{lcccccc}
\hline & Sepsis & Severe Sepsis & Septic Shock & $\begin{array}{c}\text { Sepsis vs } \\
\text { Severe Sepsis }\end{array}$ & $\begin{array}{c}\text { Sepsis vs } \\
\text { Septic Shock }\end{array}$ & $\begin{array}{c}\text { Severe Sepsis vs } \\
\text { Septic Shock }\end{array}$ \\
\hline ProCT & $3.14 \pm 7.62$ & $19.25 \pm 43.08$ & $37.15 \pm 61.39$ & $P<0.05$ & $P<0.05$ & $P<0.05$ \\
CRP & $11.46 \pm 6.99$ & $10.23 \pm 8.02$ & $14.22 \pm 6.89$ & NS & NS & $P<0.05$ \\
WBC & $11287 \pm 1667$ & $12538 \pm 7275$ & $16740 \pm 8949$ & NS & $P<0.05$ & $P<0.05$ \\
SOFA & $4.97 \pm 2.71$ & $8.23 \pm 3.33$ & $12.14 \pm 3.51$ & $P<0.05$ & $P<0.05$ & $P<0.05$ \\
MODS & $3.82 \pm 2.43$ & $6.56 \pm 3.26$ & $9.92 \pm 3.25$ & $P<0.05$ & $P<0.05$ & $P<0.05$ \\
\hline
\end{tabular}

respiratory rate, arterial pressure, WBC count, ProCT, and CRP on each ICU day. The severity of sepsis was recorded by SOFA score and the organ system failure by MODS score. Correlation of ProCT values with severity indices were done by Spearman correlation analysis and to compare independent samples we used Mann Whitney U-test. ROC curves and area under the curves of ProCT, $\mathrm{CRP}$, and WBC in diagnosing infection and prognosticating sepsis outcome, were calculated.

Compared with CRP, ProCT had a higher sensitivity ( 0.35 vs 0.73 ) and specificity (0.42 vs 0.81 ) in identifying infec- tion in SIRS with a best cutoff level of $1.31 \mathrm{ng} / \mathrm{ml}$ for ProCT and $13.9 \mathrm{mg} / \mathrm{dl}$ for CRP. Parallel to SOFA and MODS scores, there was a consistent increase in ProCT levels with sepsis severity (Table 1).

Plasma ProCT values were higher in nonsurvivors in septic patients, and area under the ROC curve of ProCT in prognosticating outcome was higher than for both CRP and WBC. The present data demonstrated ProCT as a better diagnostic marker of infection in patients with inflammatory response. This laboratory procedure also seemed to be closely correlated with sepsis severity and outcome.

\section{P72 Procalcitonin serum concentrations do not differentiate severity of septic shock in postoperative} patients

M Weiss*, E Barth*, G Fischer*, H Wiedeck*, EM Schneider*, M Georgieff* and G Steinbach ${ }^{\dagger}$

Departments of ${ }^{*}$ Anesthesiology and ${ }^{\dagger}$ Clinical Chemistry, University Hospital, D-89070 Ulm, Germany

Objectives: To investigate whether procalcitonin (PCT) serum concentrations differentiate severity of disease in postoperative/post-traumatic patients with septic shock, in which severity of shock is defined by different ranges of dosages of norepinephrine, necessary for cardiovascular stabilization.

Methods: Over a six month period, 192 patients admitted to the intensive care unit (ICU) were studied. Out of them, 18 patients were in a first phase of septic shock lasting at least four days. PCT serum concentrations in these patients were measured on days $1,2,4,6,8,10,12$ and 14 after onset of septic shock until death or discharge from ICU. PCT was measured using a commercial immunoluminometric assay (BRAHMS Diagnostica, Berlin).
Results: Nine patients survived the episode of septic shock lasting 4-10 days. Nine patients died after 5-55 days. In six patients, maximal dosage of norepinephrine was $\leq 0.1 \mu \mathrm{g} / \mathrm{kg} \times \min$ (group 1), in five patients between $>0.1$ and $\leq 0.2 \mu \mathrm{g} / \mathrm{kg} \times \min$ (group 2), and in seven patients $>0.2 \mu \mathrm{g} / \mathrm{kg} \times \min$ (group 3 ). Ranges of PCT serum concentrations overlapped between group 1,2 , and 3 , with $1.2-67.6,1.8-194.3$, and $0.4-46.1 \mathrm{ng} / \mathrm{ml}$ of PCT, respectively, resulting in no significant differences of PCT values during septic shock between the three groups.

Conclusions: PCT serum concentrations are not a marker of severity of disease in patients with septic shock, if severity of shock is defined by certain ranges of dosages of norepinephrine.

\section{P73 Expression and regulation of procalcitonin in different human cells}

\section{S Rußwurm, I Stonans, M Wiederhold, M Oberhoffer, M Meisner, PF Zipfel and K Reinhart}

Clinic of Anesthesiology and Intensive Care Medicine, University of Jena, Bachstr. 18, D-07740 Jena, Germany

Introduction: Procalcitonin (PCT) was recently forwarded as a diagnostic marker of systemic bacterial infection and sepsis. The biological function(s) and biochemical properties of this protein are poorly defined and the cellular sources of plasma PCT remains yet to be established.
Methods: Primary human cells (peripheral blood monocytes, umbilical vein endothelial cells) and cell lines (liver, renal parenchymal and lung fibroblastic lines) were cultivated under standard conditions. Basal and stimulated mRNA expression of PCT was investigated using a semi- 
quantitative reverse transcriptase polymerase chain reaction (RT-PCR). Intracellular PCT protein expression was verified by Western blotting and surface-enhanced laser desorption/ionization (SELDI). Experiments elucidating the intracellular location of PCT were performed after protein fragmentation in different fractions by secondary immunofluorescence and laser scan confocal microscopy.

Results: (1) A basal and inducible mRNA expression of PCT was found only in human peripheral blood monocytes. (2) In these cells, a distinct influence of various proinflammatory mediators was observed. (3) Western blotting of monocyte lysates using various primary antibodies directed against PCT showed a strong intracellular protein expression. (4) Experiments with SELDI revealed a molecular weight for PCT in monocytes of $12.1 \mathrm{kDa}$. (5) Human monocytes express PCT protein in association with cytoskeleton. No PCT was found in cytoplasmic fractions.

Conclusions: Since human peripheral blood monocytes produce PCT and its expression depends strongly from sepsis-related mediators, we conclude, that this cell population is one important source of elevated PCT serum levels during sepsis. Further experiments analyzing the role of Kupffer cells and liver parenchymal cells are in progress.

\section{P74 Procalcitonin helps to discriminate between septic and non-septic underlying disease at admission in ICU}

C Lepousé, B Hamou Ouali, J Cousson, P Raclot, JL Suinat and A Leon

Hôpital R. Debré, 51092 Reims cedex, France

Introduction: Differentiation between acute bacterial infection from other types of inflammation is often difficult in ICU. Procalcitonin (PCT) was reported to be a new potential specific marker for infection. The aim of this study was to assess, at the admission, a PCT cutoff of infection in ICU patients.

Methods: In a prospective study, we studied all patients admitted to our ICU between January 1999 and July 1999. PCT was measured at admission (JO) and on second day (J2). Patients were grouped according to Bones classification (SIRS, sepsis, severe sepsis, septic shock, SDMV). Statistical analysis was performed using SYSTAT (GENDEL).

Results: 377 consecutive patients (234 males and 143 females, mean age $60.3 \pm 16.9$ years, mean IGS II $34.12 \pm 17.3$ ) were admitted to ICU. Mortality rate was $26 \%$, mean length of ICU stay was 7.14 \pm 8.1 days. At admission and J2, mean PCT for all of them was $16.4 \pm 57 \mathrm{ng} / \mathrm{ml}$ and $27.1 \pm 100 \mathrm{ng} / \mathrm{ml}$. According to Bones classification, mean

\begin{tabular}{lccc} 
& Sensitivity & Specificity & $\begin{array}{c}\text { Predictive } \\
\text { positive value }\end{array}$ \\
\hline J0: PCT $\geq 1.5$ & $68.1 \%$ & $86.5 \%$ & $73.8 \%$ \\
J0: PCT $\geq 3$ & $58.6 \%$ & $92.7 \%$ & $81.9 \%$ \\
J2: PCT $\geq 1.5$ & $73.5 \%$ & $65 \%$ & $59.8 \%$ \\
J2: PCT $\geq 3$ & $67.8 \%$ & $74.7 \%$ & $65.5 \%$ \\
\hline
\end{tabular}

PCT was: without $\mathrm{SIRS}=1.74 \mathrm{ng} / \mathrm{ml} \quad(\mathrm{n}=213), \quad \mathrm{SIRS}=$ $1.51 \mathrm{ng} / \mathrm{ml}(\mathrm{n}=33)$, sepsis=14.48 $\mathrm{ng} / \mathrm{ml}(\mathrm{n}=43)$, severe sepsis=35.56 ng/ml ( $n=41)$, septic shock $=70.85 \mathrm{ng} / \mathrm{ml}$ $(n=30), S D M V=94.95 \mathrm{ng} / \mathrm{ml}(n=13)$. The PCT cut off of infection (Bones $\geq$ sepsis) is shown in the Table.

Discussion: At admission to ICU, PCT seems to be an interesting marker of early diagnosis of infection when the level is higher than $1.5 \mathrm{ng} / \mathrm{ml}$. On the second day, a PCT level of $3 \mathrm{ng} / \mathrm{ml}$ seems to be more effective for diagnosis of infection than $1.5 \mathrm{ng} / \mathrm{ml}$.

\section{P75 Procalcitonin concentrations in blood after long-distance running}

A Leon*, C Lepousé*, T Floch*, X Schwartz* and N Schneider**

*Laboratoire d'Anesthésie-Réanimation, Faculté de Médecine and ${ }^{* *}$ Laboratoire de Radioanalyse et Immunologie, Institut Jean

Godinot, Reims, France

Incidence of injuries and other health problems is known during prolonged running and recent studies suggest that exercise-induced damage may trigger production of proinflammatory molecules.

The aim of the study: The present study investigates to what extent prolonged strenuous exercise influences the plasma concentrations of procalcitonin as well as cytokines inhibitors and anti-inflammatory cytokines.
Methodology: After informed consent, 4 marathon runners and 10 half-marathon runners were recruited and investigated $12 \mathrm{~h}$ before running, immediately after the end of the run and $24 \mathrm{~h}$ later. Blood samples were collected at each time and PCT concentrations in plasma were measured with a luminometric method (Brahms). The plasma concentrations of TNF $\alpha$, IL-1 $\beta, \mathrm{IL}-6, \mathrm{IL}-1$ ra, sTNF-r1, sTNF-r2 were measured by ELISA. Statistical analysis used Systat (Jandel). 
Results: The mean duration for marathon running was $235 \pm 18 \mathrm{~min}$ and $105 \pm 10 \mathrm{~min}$ for half-marathon running. The highest concentration of IL- 6 and TNF $\alpha$ was found immediately after the run. Time course $(1=12 \mathrm{~h}$ before; 2 $=$ immediately after and $3=24 \mathrm{~h}$ later) of blood PCT, $\mathrm{ng} / \mathrm{ml}$ is presented in the figure $(P<0.001)$.

Discussion: This study suggests that long-distance running may trigger production of TNF $\alpha$ and pro-inflammatory cytokines, which stimulate the production of PCT. As it has been demonstrated in sepsis, PCT may be an early marker of elevated cytokines and muscle and/or splanchnic damage.

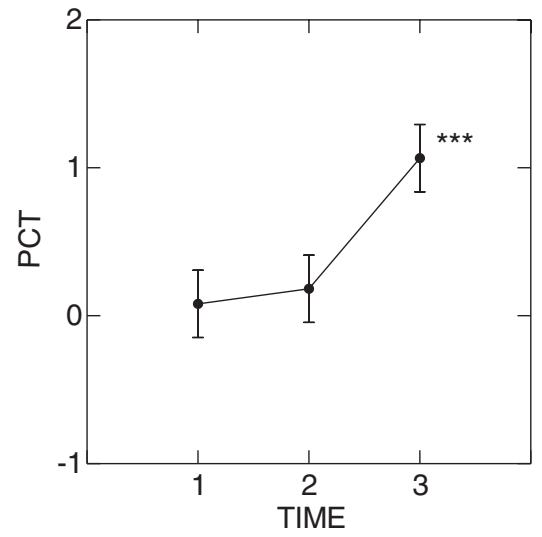

P76 The role of gallium-67 scintigraphy in diagnosing sources of fever in critically ill patients in ICU

I Rozet, S Ben-Haim* and S Burzstein-De Myttenaere

Intensive Care Unit, Department of Anesthesia, and *Nuclear Medicine, Carmel Medical Center, Haifa, Israel

Introduction: The role of radionuclide imaging to diagnose sources of infection is rather confusing [1].The aim of this study was to evaluate the diagnostic value of gallium-67-scintigraphy in critically ill febrile patients and its impact on treatment and outcome.

Methods: Forty whole-body Ga-67 scans performed in 37 patients in a five year period (1994-1999) were retrospectively analyzed.

Result: 34 patients were surgical and 3 medical with a median age 69 years and median APACHE II 23. All patients were febrile with systemic inflammatory response syndrome (SIRS) and all, except two, were ventilated for more than three days. Ga-67 scan was performed either within the first two weeks (median 7 day) because of no improvement of sepsis (group 1) or later than two weeks (median 22 day) because of the resurgence of SIRS (group 2). Group 1 consists of 24 patients with APACHE II 21, multisystemic organ failure (MSOF) in 6 patients and 5 exitus. Four from the $24 \mathrm{Ga}-67$ scans showed positive findings (two soft tissue abscesses, one intra-abdominal collection and one osteomyelitis) leading to changes in treatment and improvement of outcome. Group 2 consists of 13 patients with an APACHE II 24, MSOF and exitus in 7 patients. On the 16 Ga-67 scans performed in this group, only one showed retrosternal Ga fixation and had no impact on treatment and outcome.

False negative results were observed in 9 of 15 patients with known lung and thoracic pathology proved by other investigations. In spite of rather high sensitivity in patients with abdominal pathology, the results were non-specific.

Conclusion: Ga-67 scan in the critically ill may be helpful in localizing a pyrogenic focus in severe unimproving septic patients in the early phase after their admission. However, in patients with resurgence or sepsis in the late phase of hospitalization, Ga-67 scan appeared to be of very little diagnostic help and its value remains questionable.

Reference:

1. Peters A: Br J Radiol 1998, 71:252-261.

\section{P77 Bacteremia and systemic inflammatory response syndrome (SIRS) in confirmed post-partum} endometritis

P Myrianthefs, K Varvaresou, C Ladakis, S Pactitis, V Lappas, J Stamatiou, A Carousou, J Pavlides and J Baltopoulos Department of Intensive Care Unit, "IASO" Maternity Hospital, Kifissias 37-39, Marousi, Athens, Greece

Background: Postpartum endometritis is the most common infectious complication of pregnancy, which has a serious impact on women's health and can lead to severe sepsis.

Material and methods: We encountered 150 women with clinical evidence of early (1-48 h) post-partum endometritis and SIRS (fever, tachycardia, tachypnea and increased white blood cell count and C-reactive protein) after either vaginal delivery or caesarian section. We obtained blood cultures (aerobic and anaerobic) at $38.5^{\circ} \mathrm{C}$ and before administration of antibiotics.

Results: Fifteen samples (10\%) were positive proving bacteremia of which $12(80 \%)$ were indicative of aerobic and $3(20 \%)$ of anaerobic bacteria. There were also 7 cases of contamination due to Staphylococcus epidermidis. Isolated aerobic Gram negative bacteria 
(10/15, 66.6\%) were Escherichia coli (6), Pseudomonas cepacia (1), Enterobacter aerogenes (1), Salmonella group C (1), Klebsiella pneumoniae (1) and Gram positive cocci $(2 / 15,13.3 \%)$ were Enterococcus faecalis (1) and Streptococcus group A (1). Anaerobic bacteria $(3 / 15,20 \%)$ were Bacteroides fragilis (2) and Clostridium sp (1). All patients were treated empirically until antibiotic sensitivity tests were available with ceftriaxone $2 \mathrm{gr} \times 1$ iv, netilmicin $300 \mathrm{mg} \times 1$ iv and metronidazole $500 \mathrm{mg} \times 3$ iv. Outcome was successful in all patients. None of the isolated bacteria was multi-drug resistant.

Conclusion: Bacteremia in early post-partum endometritis was confirmed in $10 \%$ of cases. Isolated bacteria were similar to those referred to in the literature $(80 \%$ Gram negative bacteria, 20\% Gram positive and anaerobic bacteria). Early recognition and appropriate management ensures successful outcome and prevention of complications such as severe sepsis.

P78 Evolution of micro-organisms isolated in nosocomial bloodstream infections in Belgian Intensive Care Units, 1992-1999

B Jans, C Suetens, H Carsauw, I Morales, E Leens and P Selway

Scientific Institute of Public Health (IPH), J. Wytsmanstreet 14, 1050 - Brussels, Belgium

Objectives: To compare the distribution of micro-organisms from nosocomial bloodstream infections (BSIs) in Belgian intensive care units (ICUs) during two surveillance periods: from October 1992 until June 1996 and from October 1998 until September 1999.

Methods: Since 1992, Belgian hospitals have been invited to participate on a voluntary basis in the national surveillance of nosocomial BSIs (NSIH). Surveillance data are transmitted by the hospitals to the IPH for analysis, national benchmarking and feedback. The surveillance was discontinued in July 1996 and started up again on 1 October 1998. We compared the distribution of pathogens isolated in BSls occurring $>48 \mathrm{~h}$ of hospital stay before and after the interruption of the surveillance.

Results: During the first period a total of 3563 isolates were reported from 104 hospitals, for the second period 925 isolates from 62 hospitals. Table 1 shows the distribution of the micro-organisms by type. The most frequently isolated micro-organisms were coagulase-negative staphylococci (1992-1996: $22.6 \%$ and 1998-1999: $22.8 \%)$, Staphylococcus aureus $(13.1 \% ; 11.3 \%)$, enterococci $(7.8 \% ; 9.8 \%)$, Escherichia spp $(7.7 \% ; 8.6 \%)$, and Enterobacter spp $(7.1 \% ; 9.4 \%)$. The proportion of Enterobacter spp. increased significantly from $7.1 \%$ in $1992-1996$ to $9.4 \%$ in $1998-1999(P=0.017)$. This increase was due to an increase of Enterobacter aerogenes isolates (from $3.2 \%$ to $4.7 \%, P=0.025$ ). The pro-
Table 1

Distribution of micro-organisms isolated in nosocomial BSl's in Belgian ICUs, 92/96 and 98/99:

\begin{tabular}{lccccc}
\hline & \multicolumn{2}{c}{$1992-96$} & & \multicolumn{2}{c}{$1998-99$} \\
\cline { 2 - 3 } \cline { 5 - 6 } Type & $\mathrm{n}$ & $\%$ & & $\mathrm{n}$ & $\%$ \\
\hline Gram-positive cocci & 1751 & 49.1 & & 454 & 49.1 \\
Enterobacteriacea & 1030 & 28.9 & & 289 & 31.2 \\
Other gram-negative bacilli & 470 & 13.2 & & 120 & 13.0 \\
Yeast and fungi & 259 & 7.3 & & 52 & 5.6 \\
Gram-positive bacilli & 47 & 1.3 & & 8 & 0.8 \\
Other micro-organisms & 6 & 0.2 & & 2 & 0.2 \\
Total & 3563 & $100 \%$ & & 925 & $100 \%$ \\
\hline
\end{tabular}

portion of Enterobacter cloacae isolates remained constant $(3.1 \%$ to $3.6 \%)$. A significant decrease was observed for Citrobacter spp (0.9\% to $0.2 \%, P<0.03)$ and Acinetobacter spp (2.2\% to $1.2 \%, P=0.043)$.

Conclusions: Overall, the distribution of micro-organisms in BSIs in Belgian ICUs has remained quite stable since 1992. The earlier reported increasing trend in Enterobacter aerogenes BSls was again confirmed.

P79 Ten years of blood cultures surveillance in an intensive care unit of a tertiary hospital in Israel

\section{R Kitzes*, S De Myttenaere-Bursztein, A Sheskin and Z Stoler}

${ }^{*}$ Clinical Pharmacology and Infectious Diseases Unit, Intensive Care Unit\#, Department of Community Medicine and Epidemiology Carmel Medical Center, The Rappaport Faculty of Medicine, Technion, Haifa, Israel

Carmel Medical Center is a tertiary hospital with 430 beds. The intensive care unit (ICU) has 7 beds and admits patients from all hospital departments. The objectives of this study were to analyze the etiologies of blood cultures $(\mathrm{BC})$ in ICU and to compare them to those obtained from the departments. The study period included retrospectively ten years from 1988 to 1998 , excluding 1990. During this period 4497 positive BC in different patients were reported from the hospital. 3976 BC including 835 from ICU were analyzed. These BC 
represent $90 \%$ of most commonly found Gram positive and negative bacteria. The distribution of Gram negative/Gram positive BC was respectively $61 \% / 39 \%$ for the ICU and $53 \% / 47 \%$ for the departments. This difference is statistically significant. In decreasing order of incidence the most important bacteria in the ICU were: Klebsiella 23.8\%, Pseudomonas 15.6\%, MRSA 11.9\%, MRSE $10.5 \%$, Acinetobacter $8.7 \%$, Stapylococcus aureus $6.7 \%$, E. coli $5.4 \%$, Enterococcus $4.7 \%$ and Enterobacter $4.7 \%$. The most important bacteria from departments were: E. coli 19.9\%, Klebsiella 13.2\%, Staphylococcus aureus 11.4\%, Streptococcus 9.2\%, MRSE 8.2\%, Pseudomonas 7.1\%, Acinetobacter 5.3\%,
Enterococcus 5.2\%, Staphylococcus epidermidis 4.9\%, MRSA $4.1 \%$.

Conclusion: In spite of continuous transfer of patients from departments to ICU and from ICU to departments, the etiology of BC is different. E. coli and Staphylococcus aureus are the most predominant Gram negative/positive bacteria from the hospital departments, reflecting community acquisition. Klebsiella and MRSA are the most predominant Gram negative/positive bacteria in the ICU. The etiologies of $B C$ remain stable throughout the years for the majority of bacteria excluding outbreaks of MRSA and Acinetobacter in the ICU.

\section{P80 Intraluminal antibiotic treatment of central venous catheter infection in patients receiving total parenteral nutrition}

\section{Alivizatos, S Rathosis, D Skarpetas and P Athanasopoulos \\ Department of Surgery, Nutrition Unit, "St. Andrew" General Hospital, Patras, Greece}

Catheter guidewire exchange has been suggested as an effective method of treatment of catheter-related infection (CRI) in nontunneled central venous catheters. However, the old line is usually intraluminally colonised, so contamination of the new line through the guidewire occurs early. To minimise this event, we evaluated a method of treatment consisting of catheter guidewire exchange followed by intraluminal antibiotic administration.

Methods: Eight consecutive patients with CRI were studied. They received TPN through a subclavian vein catheter for a period of 8-19 days prior to CRI. In the suspicion of $\mathrm{CRI}$, the catheter was removed and exchanged over a guidewire with a new catheter, followed by catheter tip culture and peripheral blood culture. TPN administration was stopped for 2-3 days until isolation and identification of the responsible bacteria. After identification of the bacteria, TPN administration was started again and the appropriate antibiotic, as indicated by microbiological sensitivity, was administered through the new central venous catheter for a period of
10 days. All new catheters were removed after 10 days and cultured.

Results: In all the patients was noted defervescence of the clinical signs of infection following catheter removal. The responsible pathogens were Staphylococcus (S. epidermidis in $4, S$. aureus in 3, all sensitive to vancomycin), and Enterococcus in one (sensitive to ampicillin). There were no further problems with the new catheters in 7 patients, and the new cultures were sterile after 10 days of intraluminal antibiotic treatment; however, one patient developed a new episode of CRI in the 9th day of treatment, and the catheter tip culture showed colonisation by Proteus. In this patient, CRI was finally treated by removal of the catheter and replacement at a different site.

Conclusion: Our results suggest that, in the majority of cases, CRI can be successfully treated by exchange of the catheter over a guidewire followed by administration of the appropriate antibiotic through the new catheter for at least 10 days.

\section{P81 Urgent orotracheal intubation induces transient bacteremia in critically ill patients}

BJA Rijnders, A Wilmer, J Van Eldere and E Van Wijngaerden

UZ gasthuisberg, Inwendige ziekten, Herestraat 49, B-3000 Leuven, Belgium

Objectives: To test the hypothesis that urgent or semiurgent orotracheal intubation can induce short-lived bacteremia with oral flora. To find predictive factors for post-intubation bacteremia.

Methods: Prospective study in a 17 bed medical ICU. Patients in need of orotracheal intubation (OI) could be included if no cardiopulmonary resuscitation was performed. One aerobe and anaerobe blood culture (BC) was taken immediately before $\mathrm{OI}$, as soon as possible (preferable less than $10 \mathrm{~min}$ ) after and $60 \mathrm{~min}$ after OI. If it was impossible to take a $B C$ before intubation, patients could be included if both BCs after Ol were taken. The indication for $\mathrm{Ol}$, ease of $\mathrm{Ol}$, experience of the doctor $(<10,<100$ or $>100 \mathrm{Ol}$ previously) and the antibiotics used before OI were registered.

Results: During the study period (16 weeks) 70 patients underwent OI. Twenty-eight patients were included. Lack of inclusion was always due to the urgency of the situation, which caused delay in taking the first post-intubation BC. Four patients $(4 / 28$ or $14 \%)$ had streptococcal bac- 
teremia immediately after OI (mean of $11 \mathrm{~min}, \mathrm{SD} 2.5$ ). No patient $(0 / 28)$ had streptococcal bacteremia $60 \mathrm{~min}$ after OI $(P=0.03)$. Three of the four patients showed streptococcal bacteremia after $\mathrm{OI}$ had to be performed by a second doctor because of difficulties experienced during Ol whereas this was the case in only $4 / 24$ in the control group $(P<0.001)$. Two of the four doctors intubating the four patients with subsequent streptococcal bacteremia had previous experience of fewer than 10 Ols. Four of 24 doctors in the control group had previous experience of less than $10 \mathrm{Ols}(P=0.001)$. In other terms, three of the seven patients $(43 \%)$ that needed to be intubated by a second doctor due to difficulties during Ols showed transient streptococcal bacteremia.

Conclusion: Non-elective (urgent or semi-urgent) OI can cause transient bacteremia with Streptococcus species in a significant proportion of medical intensive care patients. The observed frequency of bacteremia is higher than previously reported after elective $\mathrm{Ol}$. The difficulty of $\mathrm{Ol}$ might be a major predisposing factor.

P82 Tetanus - following up 285 patients in ICU

\section{JS Brauner, SRR Vieira and CA Franke}

ICU at Nossa Senhora da Conceição Hospital and ICU at Clinicas Hospital of Porto Alegre, Porto Alegre, RS, Brasil

Introduction: Tetanus is a toxic disease generally prevented by immunization, but it is still present in the Third World and it shows high mortality rate.

Objective: To evaluate morbidity and mortality of tetanic patients in ICU during 18 years of follow up.

Methods: This trial is an historical and a prospective cohort that studied 285 patients from October 1981 to October 1999. They were classified in two groups according to modified Ablett's scale: not severe (mild and moderate) tetanus and severe tetanus. The following variables were compared: incubation period (IP), onset period (OP), symptomatic period (SP), age, period of autonomic instability (Al), period of administration of benzodiazepine (Pbenzo), curare (Pcur), mechanical ventilation (PMV), clinical and infectious events and mortality during ICU follow up. In statistical analyses, the continuous variables were presented as the mean and standard deviation. Student $t$ test was used to compare the two groups. The level of significance was $P<0.05$.
Results: This trial evaluated 71 patients with not severe tetanus and 214 patients with severe tetanus. The group with severe tetanus had a smaller IP and OP $(P=0.002$ and $P=0.003$, respectively), but a longer Pbenzo, Pcur and PMV $(P=0.001)$ than the not severe group. There was no difference concerning the mean age between the two groups $(P=0.26)$. The most common clinical events in tetanic patients were cardiorespiratory arrest $(25.6 \%)$, pneumothorax (10.17\%) and acute renal failure (10.17\%). The incidence of respiratory, urinary and catheter infections were $76.14 \%, 38.24 \%$ and $7.71 \%$, respectively. The mortality rates were $4.28 \%$ in not severe tetanus and $28.9 \%$ in severe tetanus $(P<0.001)$.

Conclusions: The severe tetanus group presents a high mortality rate, probably as a result of autonomic instability, despite intensive care. Infections related to prolonged mechanical ventilation and invasive procedures were the most frequent events noticed in this study.

P83 Leptospirosis in intensive care units: report of 33 cases

SRR Vieira, JS Brauner, F Fernandes and C Moraes

ICU at Clinicas Hospital of Porto Alegre and ICU at Nossa Senhora da Conceição Hospital, Porto Alegre, RS, Brasil

Introduction: Leptospirosis is, in general, a self-limited disease but it can be associated with important complications such as multiple organic dysfunction and high mortality [1].

Objective: The goal of this paper is to evaluate the clinical characteristics and the morbimortality of severe leptospirosis in general Intensive Care Units from two general hospitals.

Methods: All cases with the diagnosis of leptospirosis confirmed by blood macroagglutination test and admitted from 1990 to 1999 were studied. We have analyzed their clinical and laboratory characteristics, the occurrence of multiple organ dysfunction and their mortality rate. We have also compared survivors with non-survivors. The quantitative variables have been compared by unpaired $\mathrm{t}$-tests and the qualitative variables by a Chi squared test.

Results: We described 33 adult patients, aged $40 \pm 16$ years, of which 27 were men and 6 women. The most frequent clinical manifestations were fever $(n=30)$, myalgias $(n=29)$, jaundice $(n=27)$ and dyspnea $(n=27)$. All patients showed some level of organic dysfunction such as respiratory $(n=29)$, renal $(n=24)$, hepatic $(n=24)$ and cardiovascular $(n=20)$. The mortality rate was $52 \%(n=17)$. The comparison of non-survivors with survivors showed they have higher incidences of respiratory, renal, cardiovascular 
and neurological failures as well as higher levels of acidosis $(P<0.05)$.

Conclusions: In endemic regions leptospirosis has to be considered as a cause of multiple organic dysfunction with a high mortality rate mainly when respiratory, renal, cardiovascular or neurological failures are present.

References:

1. Ko Al et al:: Urban epidemic of severe leptospirosis in Brazil. Lancet 1999:820-825.

\section{P84 Falciparum malaria in ICU}

A Aaron and B Avinash

Parsee General Hospital, Cumballa Hill, Mumbai 400036, India

Objectives: To study and compare the various treatment modalities and the attendant complications of Falciparum malaria with or without concomitant Vivax in the Intensive Care Unit between 1993 and 1999.

Methods: Retrospective study of patients hospitalized between 1993 and 1995 and prospectively from 1996 to 1998.

Results: See Table.

Conclusions: 1) Over the period of years, early use of Quinine has given good and consistent results $(10 \mathrm{mg} / \mathrm{kg}$, up to $600 \mathrm{mg}$ thrice daily for 5-10 days) and can cure all stages of Falciparum and other Plasmodia, save the hyp- nozoites of Vivax. 2) Parasites have developed resistance to Chloroquine, Sulfadoxine- Pyremethamine. Quinine is poorly tolerated when given after Mefloquine due to the addition of adverse effects especially those of GIT. 3) If patients who have been treated with chloroquine or other drugs, including if radical cure has been attempted, continue to spike, it is best to restart with quinine even if a species other than Falciparum is the culprit. 4) Hypoglycemia, otological complications were not really severe enough or irreversible to warrant stopping Quinine. Although ECG(QTc) or other cardiac disturbances were not seen in those group,a daily ECG is a must. 5) In severely toxic patients, a simultaneous intramuscular dose of $60 \mathrm{mg}$ of Artesunate on each buttock followed by a daily dose of $60 \mathrm{mg}$ for the next 5 days reduces the para-

Table

Total admissions in the ICU:

Quinine group: 40 patients

Survived

34

All Started with Quinine $10 \mathrm{mg} / \mathrm{kg}$

(up to max $600 \mathrm{mg}$ thrice a day for 5-10 days)

IV Fluids esp. Dextrose

Antibiotics esp. Doxycycline

96
Non Quinine group: 56 patients

Survived $20 \quad$ Died 36

Started either with Chloroquine, SulfadoxinePyremethamine, Mefloquine with or without Halofantrine, Doxycycline, Norfloxacin Artemesnin derivatives and others

Other parameters like Mechanical ventilatory support, Blood transfusions, Positive lonotropes were used indiscriminately for all patients, as the situation demanded

Complications:

Complications:

CNS - Drowsy, altered sensorium responded well by the 3rd dose of Quinine

CNS - Disorientation, Unconsciousness, coma, death

Respiratory- Reversible with prompt treatment Respiratory - Respiratory Failure, ARDS and others recovered well after mechanical ventilaton

Renal Failure: High Creatinine due to microvascular ischemia returned to baseline over a period of time

Renal Failure - High creatinine, Anuria, ATN

DIC, Shock, Multi organ failure were not encountered

Hepatic failure, Multi Organ Failure, Hemolysis, Circulatory failure, DIC Death

Ominous signs of Falciparum

1) Peripheral smear: due to invasion of young RBCs and sequestration in tissues, a large number of parasites may be invisible on the smear, thereby giving false assurance of a "low parasite index".

2) Premunition: in holo and hyperendemic areas, due to some degree of acquired immunity, patients are largely asymptomatic early on. It is almost always late when symptoms manifest overtly.

3) Cerebral Malaria is a "diffuse symmetrical encephalopathy" and therefore sparing mild neck rigidity, signs of meningeal irritation are largely absent. Mild delerium, obtundation, convulsions etc. are to be taken seriously.

4) Pregnant women are spared gross parasitemia for a long time due to heavy "parasitisation of the placenta". 
site load as it is relatively new and thereby less resistant and is an effective schizonticide. It is, however, devoid of any action on the gametocytes. This was the only other group of drugs which promised future potential for concomitant use with Quinine. 6) It is best to wait for a minimum $72 \mathrm{~h}$ before discontinuing quinine or switching to an alternate regime. 7) Intravenous Quinine may be used only in the most toxic and orally intolerant population. 8) Primaquine must be given in Chloroquine resistant Vivax even after usage of Quinine for radical cure.

\section{P85 Fungaemia in the intensive care unit: Baragwanath experience}

\section{B Zeippen and S Madhi}

Intensive Care Unit, Chris-Hani Baragwanath Hospital, University of the Witwatersrand, Johannesburg, PO Bertsham 2013, RSA

Introduction: Despite advances in the care of the critically ill, fungaemia often remains undiagnosed and caries a high mortality.

Objectives: To assess the demographics, the morbidity and mortality of patients infected with Candida species.

Materials and methods: The intensive care unit records of 26 patients diagnosed with fungaemia during 1998 were reviewed (eight medical, twelve surgical, six paediatrics).

Results: See Table.

Conclusion: Despite treatment, fungaemia carries a high mortality.The actual mortality of $69 \%$ is higher then predicted by the APACHE II/PRISM scores.

\section{Table}

Demographics:

\begin{tabular}{|c|c|c|c|c|c|}
\hline & & Total $(n=26)$ & Alive $(n=8)$ & Dead $(n=18)$ & $P$ \\
\hline Age (years) & Median (range) & 47 (8d-76y) & $26(10 m-53 y)$ & 48.5 (8d-76y) & 0.182 \\
\hline APACHE $\|(n=20)$ & Mean (SD) & $16.9(7.4)$ & $11.6(6.1)$ & $18.6(7.0)$ & 0.06 \\
\hline PRISM (n=6) & Mean (SD) & $12.7(5.8)$ & $8.6(5.0)$ & $16.6(3.1)$ & 0.07 \\
\hline ICU stay (days) & Median (range) & $13.5(1-115)$ & $15.5(3-71)$ & $11(1-115)$ & 0.88 \\
\hline Delay to Rx (days) & Mean (SD) & $2.6(3.6)$ & $8.0(1.5)$ & $1.2 \mathrm{~d}(2.6)$ & $<0.05$ \\
\hline
\end{tabular}

Microbiology:

Mean time to initial fungaemia following ICU admission was 6.5 days (25th-75th: $2-14)$. The mean time of isolation of fungi from tracheal aspirate $(n=9)$, catheter tip $(n=7)$, urine $(n=6)$ was 4.4 (SD 4.4), 12.3 (SD 13) and 21.8 (SD 18.2) days respectively.

\begin{tabular}{|c|c|c|c|c|c|}
\hline \multirow[b]{2}{*}{ Albicans } & \multirow{2}{*}{$\frac{N}{10}$} & \multirow{2}{*}{$\begin{array}{c}\text { Cured } \\
3\end{array}$} & \multirow{2}{*}{$\begin{array}{c}\text { Relapse } \\
0\end{array}$} & \multicolumn{2}{|c|}{ Died during therapy } \\
\hline & & & & 7 & $70 \%$ \\
\hline Glabrata & 5 & 0 & 2 & 3 & $60 \%$ \\
\hline Parapsilosis & 10 & 6 & 0 & 4 & $40 \%$ \\
\hline Glabrata+Parapsilosis & 1 & 0 & 0 & 1 & $100 \%$ \\
\hline$\leq 5$ risk factors & 11 & 6 & 0 & 5 & $45 \%$ \\
\hline$\geq 6$ & 15 & 3 & 2 & 10 & $66 \%$ \\
\hline
\end{tabular}

\section{P86 High incidence of positive fungal DNA in the blood of critically ill patients}

\section{K Ikegami, T Sakai*, F Morimoto, T Seki, K Hirose and Y Sato}

Trauma and Critical Care Center, Koshigaya Hospital, Dokkyo University School of Medicine, 2-1-50 Minamikoshigaya Koshigaya-shi, Saitama, Japan 343-8555; *Department of Molecular Biology, Kyorin University School of Medicin, Japan

Introduction: Systemic fungal infection is not uncommon in critically ill patients. Because of difficulty in the diagnosis, early antifungal therapy is not always possible.
Although positive blood culture for fungus is well recognized as a hallmark of systemic therapy, sensitivity of blood culture is low. Detection of fungal DNA in the blood is 
expected to serve as a new clue to initiate antifungal therapy.

Purpose: To assess the incidence of positive fungal DNA in the blood of critically ill patients.

Methods: 34 blood samples were obtained from 24 patients. Blood samples were processed for polymerase chain reaction (PCR) to detect fungal DNA. DNA extraction was performed with the modified methods of Buchman. PCR primers to amplify conserved DNA sequences of $18 \mathrm{~S}$ rRNA genes shared by most clinically important fungi were used. The amplification was performed in a thermal cycler through the cycles as follows.
Results: Fungal DNA was detected from the 16 samples taken from 22 patients with SIRS and from seven samples taken from 12 patients without SIRS. When patients developed SIRS after seventh hospital day, fungal DNA was detected in $80 \%$ of samples taken from these patients.

Conclusions: Incidence of positive fungal DNA was more than $50 \%$ and the incidence reached $80 \%$ when patients developed SIRS after seventh hospital day. High incidence of positive fungal DNA suggests that there are more candidates for systemic antifungal therapy than expected.

\section{P87 Frequency, mortality and risk factors of candidemia at a tertiary care hospital}

J Garbino, P Rohner, Th Kinge, P Pichna, D Pittet and D Lew

Infectious Diseases Division University Hospital of Geneva, Geneva, Switzerland

Introduction: The incidence of fungal infections has been increasing for the last three decadess, especially in neutropenic, cancer and critically ill patients. It is associated with high mortality rates.

Methods: We retrospectively reviewed medical charts of adult patients with fungemia from 1989 to 1998 at the University Hospital of Geneva. For patients with candidemia, demographic information, risk factors (antibiotic treatment, preceding surgery, cancer, intravascular devices, ICU stay), outcome, and the incidence of fungal species was analyzed as well as the overall consumption of fluconazole.

Results: Of 291 patients with fungemia, we reviewed 278 (96\%) medical records, $184(66 \%)$ were from male patients. The mean age was 60 years and the overall mortality $45.5 \%$. Candida albicans (61\%) was the most commonly identified species followed by C. glabrata (15\%), and C. parapsilosis (6\%). The incidence of candidemia ranged from 0.2 to 0.56 per 10000 patient-days with the highest incidence in 1993 and the lowest in 1997. Of the risk factors analyzed only ICU stay was statistically significant $(P<0.0001)$ (OR 3.42; Cl 1.98-5.92). When candidemia was revealed, $38 \%$ of the patients were hospitalized in an ICU. Most candidemias occurred several days after admission, that is $52 \%$ after two weeks and $70 \%$ after three weeks.

Conclusion: The regular use of fluconazole since 1990 may explain a decreasing trend of candidemias due to $C$. albicans, whereas those due to other Candida spp remained stable. The predisposing risk factors analyzed associated with a high mortality were similar to those described in other series. For the last two years, fungemia remained low and stable in our institution. No significant changes in the incidence of fungal species during the 10 year study period were observed.

\section{P88 Hospital urinary infection epidemic by Candida famata: reduction of mortality after therapy with fluconazole and identification of the risk factors related to the infection}

MS Lapchik*, E Andrioli*, M Melo', P Junior ${ }^{\dagger}$, LC Leiro* and EF Silva*

${ }^{*}$ Hospital Evaldo Foz, Av. Vereador J. Diniz, 3505, São Paulo, Brazil, CEP-04603-901; 'Laboratório SAE, São Paulo, Brazil

Background: The more recent studies have revealed that infections by Candida spp. have increased their participation as a hospital infection. Some clinical studies have revealed reduction of the mortality rates in critical patients treated for candiduria with fluconazole. In 1997 and 1998, we detected an epidemic outbreak of hospital urinary infection by $C$. famata, which determined an epidemiological investigation.

Objectives: Identify the risk factors for hospital candiduria by C. famata, the lethality attributed to this infection, as well as evaluate the impact of early treatment with fluconazole on the lethality of candiduria.
Results: This matched paired case-control study detected 19 cases of hospital infection by C. famata, fulfilling $100 \%$ of the pairing criteria with 38 patients of the control group. The univariate analysis revealed the following variables as risk factors for hospital candiduria by $C$. famata $(P<0.05)$ : Post-operative of abdominal surgery, previous use of antimicrobials with coverage for aerobic Gram-negative bacilli and anaerobic bacteria, the prolonged use of vesicular catheter ( $>4$ days) and of antimicrobials. The greater time of use of antimicrobials was considered the only independent risk factor, obtained by multivariate analysis $(O R=1.1)$. The lethality attributed to hospital candiduria by C. famata was 
$42 \%(P<0.05)$, with identification of secondary infection in the blood stream in two patients. All the patients who did not make use of fluconazole or other anti-fungal therapy for treatment of the candiduria (7 patients) evolved to obit. $(P<0.05)$ No potential sources of infection were identified, with an endogenous source being attributed to as most probable for the occurrence of the outbreak.
Conclusion: The diagnosis of candiduria in critical patients remains as a characteristic related to the high morbidity and lethality. The institution of preventive measures such as the reduction of time of use of vesicular catheter, rational use of antimicrobials, and the early treatment of candiduria episodes in critical patients, are measures which can reduce the lethality related to this infection, considering candiduria as a predisposing factor to systemic candidiasis. The research of $C$. famata as etiological agent in hospital urinary infection must be taken into account in future studies.

\section{P89 Usefulness of vancomycin serum concentration monitoring in the critically ill patient}

M Bertolissi, A Di Silvestre, F Pea*, M Furlanut* and F Giordano

Department of Anesthesia and ICU $2^{\circ}$ General Hospital, * Department of Clinical Pharmacology University, 33100 Udine, Italy

Introduction: The aim of this study was to compare two methods, the serum concentration monitoring (SCM) and the nomogram of Moellering (NM), employed to calculate the dose of vancomycin (V) in the critically ill patient.

Methods: We studied 32 patients admitted to our ICU and treated with $\mathrm{V}$ for Staphylococcus aureus infections. The patients were randomly divided into two groups: $A(16)$ and $B$ (16). In group $A$ the dose of $V$ was calculated by the SCM (fluorescence polarization immunoassay technique) on two blood samples drawn daily just before the first administration of the drug [to determine the minimal serum concentration ( $\mathrm{C} \mathrm{min}$ )] and $30 \mathrm{~min}$ after the infusion of $\mathrm{V}$ has been stopped [to determine the maximal serum concentration ( $\mathrm{C}$ max)]. In group $B$ the daily dose of $V$ was calculated on the basis of the NM even though the SCM was also performed but its result was not known. We fixed the therapeutic range of $V$ between 5 and $10 \mathrm{mg} / \mathrm{l}$ for $\mathrm{C} \min$ [1].

Results: In group A, C min of vancomycin was inside the therapeutic range in all patients: In group $B$ it was above $10 \mathrm{mg} / \mathrm{l}$ in 4 patients $(14.3 \pm 2.7)$ and below $5 \mathrm{mg} / \mathrm{l}$ in 3 patients $(3.9 \pm 0.3)$ (Table 1). C max was $20.4 \pm 3 \mathrm{mg} / \mathrm{l}$ in group $A$ and $20.1 \pm 6 \mathrm{mg} / \mathrm{l}$ in group $B$.

Discussion: The results obtained show that the SCM is a useful tool to maintain the serum concentration (SC) of vancomycin inside the therapeutic range, while the NM is not so effective. The possibility of maintaining the right SC of $V$ is essential to prevent both the side effects from overdosing (oto, nephro-toxicity) and underdosing the drug (uneffective antibiotic activity, bacterial resistance), but it
Table 1

Number of patients with the minimal serum concentration (C min) of $\mathbf{V}$ below, between and above the limits of the therapeutic range.

\begin{tabular}{lcc}
\hline Group & A & B \\
\hline$C$ min $<5 \mathrm{mg} / \mathrm{l}$ & 0 & 3 \\
$5 \mathrm{mg} / \mathrm{l}<\mathrm{C} \min <10 \mathrm{mg} / \mathrm{l}$ & 16 & 9 \\
$\mathrm{C} \min >10 \mathrm{mg} / \mathrm{l}$ & 0 & 4 \\
\hline
\end{tabular}

can be problematic in the critically ill patient whose physiopathological characteristics may interfere with the pharmacokinetics of the drug. In the 4 patients who showed a $\mathrm{C}$ min of vancomycin above $10 \mathrm{mg} / \mathrm{l}$ we did not find any correlation between the elevated level of $\mathrm{V}$ and the clinical conditions; in the 3 cases with $\mathrm{C}$ min below $5 \mathrm{mg} / \mathrm{l}$ we observed an increase in the volumes of distribution because of peritonitis and fluid overload.

We conclude sustaining the usefulness of the serum concentration monitoring to establish the right dose of vancomycin in the critically ill patient, and underlining that the clinical conditions characterized by the increase in the volumes of distribution represent a risk factor of underdosing the drug.

\section{Reference:}

1. Leader WG et al:: Pharmacokinetic optimisation of vancomycin therapy. Clin pharmacokinet 1995, 28(4):327-342.

\section{P90 Vancomycin as a single daily dose in critically ill patients on an intensive care unit}

\section{S Grundler, N Stallard, A Paull and MN Smithies}

University Hospital of Wales, Heath Park, Cardiff, Wales, UK, CF4 4XW

Purpose: The purpose of the study was to assess the peak and trough levels of $2 \mathrm{~g}$ of vancomycin (VAN), given once daily (od), in critically ill patients, irrespective of renal function, instead of Ig VAN twice daily.
Methods: Prospective, observational, open study. $2 \mathrm{~g}$ of VAN, diluted in $500 \mathrm{mls}$ of $0.9 \%$ normal saline, infused over four hours. VAN blood-level assays were done at 1,2 and $12 \mathrm{~h}$ after completion of the infusion, and 12 hourly there- 
after or until the next dose of VAN. (Random VAN levels are done routinely on the ICU at $08 \mathrm{~h} 00$ daily.) Another dose of VAN was given if the random level was below $10 \mathrm{mg} / \mathrm{l}$.

Results: Five subjects (2 male, 3 female) aged 16-64 (mean 56士23.5) years, weighing 65-105 $(83 \pm 21.4) \mathrm{kg}$ were enrolled. The assay used to measure VAN levels was the FPIA using the FLX system. The peak levels 1 and $2 \mathrm{~h}$ after the infusion were between 21.5-50.6 (35.6 \pm 12.9 $\mathrm{mg} / \mathrm{l})$ and $20.4-48 \mathrm{mg} / \mathrm{l}$ respectively.

Conclusion: Our peak levels did not exceed $80 \mathrm{mg} / \mathrm{l}$, a level which has been associated with ototoxicity. The incidence of nephrotoxicity with VAN alone is about $5 \%$ and is unrelated to serum VAN concentrations. We did not assess the effect on renal function, since most of our patients requiring VAN were already on continuous renal replacement therapy.

Concerns have been voiced regarding the short postantibiotic effect (PAE) of VAN $(<2 \mathrm{~h})$. In aminoglycides the larger, od dose resulted in a prolonged PAE. The PAE of once daily VAN is unknown and at present we are maintaining trough levels above $10 \mathrm{mg} / \mathrm{l}$.

Our results show that od VAN $2 \mathrm{~g}$ should not cause an increase in ototoxicity as peak VAN levels are well below $80 \mathrm{mg} / \mathrm{l}$ and if trough levels are maintained above the minimum inhibitory concentration of the organism, then concerns regarding the short PAE are eliminated.

\section{P91 Risk factors associated with vancomycin-resistant enterococcal colonization in a general ICU and the effect of surveillance and prevention over respiratory tract and nosocomial infections}

\section{PS Martins, FR Perrechi, MH Kai, RC Zanella, F Valdetaro and S Blecher}

Hospital Santa Marcelina, São Paulo, Brasil

Objectives: 1) To determine the risk factors associated with vancomycin-resistant enterococcal (VRE) colonization in a general ICU. 2) To observe the effect of infection control measures over respiratory tract and nosocomial infections in that unit.

Design: Point-prevalence surveys for VRE colonization of patients were carried out over the period from March 1998 to June 1998.

Setting: General medical and surgical ICU in a tertiary teaching hospital in the city of São Paulo, Brasil.

Measurements: The first case of VRE colonization in the unit was related in March 1998. Point-prevalence surveys were then carried out to evaluate the main risk factors related to VRE colonization (enteral feeding, severity of illness, length of ICU stay, colonization pressure, antimicrobial use, and others). A univariate analysis was made and the variables that demonstrated $P \leq 0.10$ were then entered into a discriminant analysis to identify the $\beta$ coefficients.

Measures against VRE colonization were established based on CDC criteria (MMWR 44(RR12);1-13) and the effects of these measures over the respiratory tract infec- tion rate and the nosocomial infection rate according to the NNIS (National Nosocomial Infections Surveillance) system were measured. Strain typing was characterized using pulsed-field gel electrophoresis (PFGE).

Main results: Thirty-eight patients were evaluated. 18 VRE and 11 vancomycin-susceptible enterococcal (VSE) colonizations were identified in anal swab, wound and venous catheter. Out of 18 enterococcal strains isolated, 13 strains were $E$. faecium, distributed in 5 different groups according to PFGE. The other 5 strains were $E$. fecalis, distributed in 4 groups according to PFGE as well.

The results concerning the univariate analysis and the discriminant analysis (Table 1), the respiratory tract infection rates and the nosocomial infection rates (Table 2) are given.

Conclusions: The colonization pressure, vancomycin use and length of ICU stay $\geq 29$ days were the main risk factors associated with VRE colonization.

Measures against VRE colonization resulted in a significant decrease in the respiratory and nosocomial infection rates. This decrease was probably due to reduction of other pathogens colonization pressure.

Table 1

\begin{tabular}{lccc}
\hline & Univariate analysis & & Discriminant analysis \\
\cline { 2 - 3 } Risk factors & OR (Cl 95\%) & $\beta$ & Cutoff $\geq 5$ \\
\hline Length of ICU stay $\geq 29$ days & $7.07(0.92-6.77)$ & $1.9^{\star}$ & Sensitivity $94 \%$ \\
Vancomycin & $12.5(1.7-326)$ & $4.08^{\star}$ & Specificity $81.8 \%$ \\
Colonization pressure & $36(3.19-691)$ & $6.38^{\star}$ & \\
\hline
\end{tabular}


Table 2

\begin{tabular}{lccccc}
\hline & \multicolumn{2}{c}{$\begin{array}{c}\text { Respiratory infection } \\
\text { rate/1000 }\end{array}$} & & \multicolumn{2}{c}{$\begin{array}{c}\text { Nosocomial infection } \\
\text { rate/1000 }\end{array}$} \\
\cline { 2 - 3 } \cline { 5 - 6 } Months & 1997 & 1998 & & 1997 & 1998 \\
\hline January & 54 & 57 & & 210 & 160 \\
February & 66 & 65 & & 219 & 147 \\
March & 46 & 47 & & 144 & 139 \\
April & 47 & 34 & & 147 & 116 \\
May & 55 & $21^{*}$ & & 144 & $88^{\star}$ \\
June & 51 & $22^{\star}$ & & 188 & $91^{*}$ \\
July & 61 & $13^{\star}$ & & 162 & $81^{*}$ \\
August & 56 & $22^{*}$ & & 186 & $88^{*}$ \\
September & 53 & $25^{*}$ & & 221 & $85^{\star}$ \\
\hline
\end{tabular}

${ }^{\star} P \leq 0.05$.

P92 Risk factors of infection and molecular typing in ICU colonized patients with Enterobacter aerogenes

E Carlier*, M Piagnerelli*, A Deplano ${ }^{\star}$, P Lejeune* and D Govaerts ${ }^{\dagger}$

${ }^{*}$ Department of Intensive Care and ${ }^{\dagger}$ Department of Microbiology, A. Vésale Hospital, 6110 Montigny-le-Tilleul; ${ }^{\ddagger}$ Department of Microbiology, Erasme University Hospital, 1070 Brussels, ULB, Belgium

Objectives: To identify risk factors for Enterobacter aerogenes (Ea) infection in colonized intensive care patients and compare by Pulsed-Field Gel Electrophoresis (PFGE) infection strains to colonization strains.

Methods: During a prospective 8 month period, prescription order of perineal swabs has been undertaken to all ICU patients hospitalized more than $48 \mathrm{~h}$ on the admission day, discharge and on a weekly basis. The following risk factors were studied by univariate and multivariate analyses: age, sex, underlying pathologies, antibiotic and corticoid therapies, SAPS II score, intravascular and urinary catheters, surgical drain, nasogastric tube, use of mechanical ventilation, bronchoscopy, length of ICU stay, length between hospital and ICU admission (H/ICU) and mortality rate. Antimicrobial susceptibility and clonal analysis by PFGE were performed in colonized and infected groups.

Results: 204 patients were included: 170 without Ea colonization/infection (83.3\%), 24 colonized (11.8\%) and 10 infected (4.9\%). The Ea infection in colonized patients was associated with younger age: respectively 76.5 years in colonized and 63.5 years in infected patients $(P=0.039)$; higher ICU stay (9 days versus 31 days; $P=0.007)$ mechanical ventilation $(P=0.039)$ and bronchoscopy $(P=0.026)$. PFGE profiles demonstrated two major clones (profiles 1 and 2) in ICU colonized and infected groups (45/52 strains tested). We calculated an attack rate infection/colonization of $28 \%$ for clone 1 and $20 \%$ for clone 2 .

Seven of eight infected patients $(87.5 \%)$ had the same genomic profile for colonization and infection using PFGE.

Conclusions: Our results indicate that younger age, long length of ICU stay, use of mechanical ventilation and bronchoscopy were risk factors for Ea infection in colonized patients. Ea colonization is associated with a very high risk of Ea infection by the same genomic strain. This may guide antibiotic treatment in this particular population of ICU patients.

\section{P93 The new way of postoperative pulmonary complications prophylaxis in oesophageal cancer} surgery

S Sviridova, I Nekhaev, M Kiselevsky, A Pleskov and E Gorobets

Russian Cancer Research Centre, 115478, 24 Kashirskoye shosse, Moscow, Russia

Objective: The efficacy of Neupogen (granulocyte colonystimulating factor) in prevention of pulmonary complications after transthoracic subtotal oesophagectomy (TSE).

Design: Prospective randomized double-blind clinical trial. 
Materials: Twenty-eight oesophageal cancer patients were treated with Neupogen $5 \mu \mathrm{g} / \mathrm{kg}$ s.c. one day prior surgery, and on the 1 st and 2 nd days after TSE. Thirty-five patients received placebo on the same days. No one patient was basically neutropenic. The therapeutic regimen, including choice of antibacterial agents, was similar in both groups. The incidence of postoperative pneumonia (according to CPIS criteria), sepsis and multiple organ failure syndrome (MOFS) was registered.

Results: Four (14\%) patients in the Neupogen group developed pneumonia postoperatively. There were no cases of sepsis and MOFS in this group. White blood cells (WBC) in peripheral blood samples on the third postoperative day reached $24.5 \pm 2.9 \times 10^{3}$ per $\mu$ l.

On the contrary, severe pneumonia occurred in $16(46 \%)$ patients of control group including $5(14 \%)$ cases of sepsis and MOFS. WBC level was $8.1 \pm 3.4 \times 10^{3}$ per $\mu$ l. The difference between the two groups was significant $(P<0.05)$.

Conclusion: The stimulation of granulocytes with Neupogen decreases greatly the incidence of pneumonia and sepsis after transthoracic oesophageal resections in cancer patients, and probably after extended cancer surgical procedures in general.

\section{P94 The impact of late-onset ventilator-associated pneumonia on mortality in a Saudi-Arabian} hospital

W Djazmati, GA Oni, ZA Memish, G Cunningham, M Itani, Y Beyene and R Mclntyre

King Fahad National Guard Hospital, ICU 1425, PO Box 22490, Riyadh 11426, Saudi Arabia

Introduction: Ventilator-associated pneumonia (VAP) has been recognized as a major factor affecting mortality in intensive care units (ICU). This study compared the relative impact on mortality of early- versus late-onset VAP in an adult medical surgical ICU of a 550-bed tertiary care hospital in Riyadh, Saudi Arabia.

Methods: All patients mechanically ventilated for more than $48 \mathrm{~h}$ between November 1996 and December 1997 were included prospectively. VAP was diagnosed according to the definition of the Center for Disease Prevention and Control (CDC), Atlanta. Early- or late-onset VAP was defined respectively as the occurrence of VAP within or after five days of intubation. Pathogens isolated were compared between the two groups. The mortality in the two groups were also compared using univariate and multivariate (logistic regression) analysis.

Results: A total of 202 patients were included in the study. Our incidence density rate of VAP was 16.8 per 1000 person days of ventilation. Fifty-one patients
(25.2\%) developed VAP, of whom 22 (43.1\%) were lateonset. The mortality rates for the early- and late-onset VAP groups were $10.3 \%(95 \% \mathrm{Cl}=2.7$ to $28.5 \%)$ and $36.4 \%$ (95\% Cl=18.0 to $59.2 \%)$, respectively. In a univariate analysis, the relative risk of mortality in the latter group was $3.5(95 \% \mathrm{Cl}=1.1$ to $11.7 ; P=0.029)$. $S$. aureus was significantly more common in early- compared to late-onset $\operatorname{VAP}(44.8 \%$ vs $13.9 \% ; P=0.02)$, while Acinetobacter was more common in late- compared to early-onset VAP (40.9\% vs $17.1 \% ; P=0.06)$. In the logistic regression in which adjustment was made for the effects of pathogens, age and the administration of $\mathrm{H}_{2}$ receptor antagonists, late-onset VAP remained significantly associated with mortality (risk ratio $=6.0,95 \% \mathrm{Cl}=1.1$ to $33.9 ; P=0.042$ ).

Conclusion: Our study shows significant association of late-onset VAP with mortality. This supports what has been observed in previous studies. It is possible to use the time of occurrence of VAP as an important indicator of mortality, and may be used to identify 'at risk' patients and initiate more aggressive therapy at an earlier stage.

\section{P95 Effect of mechanical ventilation and respiratory tract colonization on bronchial immunoglobulin} $A$ and $G$ levels in ICU patients

\section{A Kalopissi, X Katsenos, E Kremasmenou, A Anthi, K Mandragos, K Tergoglou and P Behrakis}

Intensive Care Unit, Red Cross Hospital, Athens, Greece

Objective: The aim of this study was to evaluate the potential effect of mechanical ventilation and colonization of respiratory tract on bronchial $\lg A$ and $\lg G$ levels.

Patients and methods: Fifteen patients under mechanical ventilation, 10 males and 5 females, sustaining cerebrovascular injury, were included in the study. All patients underwent blood and bronchial immunoglobulin determinations on day 1 (intubation day) and day 4. At the same time bronchial cultures were obtained. None of the patients developed lung infection during the study period.

Results: Mean $\operatorname{lgG}$ and $\lg A$ plasma levels were 757.72 $\mathrm{mg} / \mathrm{dl}$ (day 1) $738.81 \mathrm{mg} / \mathrm{dl}$ (day 4) and $163.45 \mathrm{mg} / \mathrm{dl}$ (day 1), $185.18 \mathrm{mg} / \mathrm{dl}$ (day 4), respectively.

Mean $\lg G$ and $\lg A$ bronchial levels were $35.95 \mathrm{mg} / \mathrm{dl}$ (day 1), $29.69 \mathrm{mg} / \mathrm{dl}$ (day 4) and $17.84 \mathrm{mg} / \mathrm{dl}$ (day 1), $14.76 \mathrm{mg} / \mathrm{dl}$ (day 4), respectively. No significant differ- 
ences were observed between the two measurements of immunoglobulins (day 1,4). In contrast, bronchial cultures were positive $\left(>10^{4} \mathrm{cfu} / \mathrm{ml}\right)$ in 13 out of 15 patients on day 4 . Bronchial cultures obtained on day 1 were negative in all the patients.
Conclusion: The colonization of the respiratory tract occurring under mechanical ventilation doesn't alter significantly the bronchial immunoglobulin levels. Although the slight decrease observed may account for the high incidence of pneumonia in ICU patients.

\section{P96 Effects of bronchoalveolar lavage (BAL) fluids of patients with ventilator-associated} bronchopneumonia (VAP) on alveolar cells in culture

M Nys*, P Damas*, G Deby*†, M Mathy', S Legrand‡ and M Lamy*†

*Service of Anaesthesiology and Intensive Care, University Hospital of Liege; ${ }^{+}$Center of Oxygen Research and Development, University of Liege; ' Laboratory of Fundamental Virology, University of Liege, B4000, Sart-Tilman, Liege, Belgium

Lungs of patients with VAP or ARDS are invaded by neutrophils which are activated and degranulate in situ, releasing granulocytic enzymes and reactive oxygen species (ROS) that could be responsible for alterations of neighbouring alveolar cells. When clinical and radiological signs suggesting VAP were encountered, we performed 71 bronchoalveolar lavages (BAL) in 58 ventilated patients (42 men, 16 women with a mean age of $55.59 \pm 16.87$ years; an APACHE II score at entry of $15.85 \pm 5.49$; a mean intubation duration of $19.93 \pm 15.49$ days; a mean ICU stay of $29.45 \pm 23.99$ days; and 17 deaths). VAP was confirmed by quantitative bacteriological culture $>104 \mathrm{CFU} / \mathrm{ml}$ of $\mathrm{BAL}$ and non-infectious ARDS was confirmed by classical criteria including $\mathrm{PaO}_{2} / \mathrm{FiO}_{2}$ ratio <200. In these $\mathrm{BAL}$ fluids, we measured the concentrations of nitrated proteins (NTP) as an indicator of oxidative activity (ELISA technique), and the concentrations of active myeloperoxidase and elastase, two markers of phagocyte degranulation (enzymatic measure). The effects of BAL fluid on human alveolar cells (A549) in culture were analyzed by measuring the release of ${ }^{51} \mathrm{Cr}$ pre-incorporated by the cells (cytotoxicity test) and correlated with NTP, myeloperoxidase and elastase values (Pearson's correlation and Mann-Whitney test with $P<0.05$ being significant). Preliminary studies were also performed to determine the mechanism of cytotoxicity of BAL by analysing the capacity of these fluids to activate the nuclear transcription factor NFKB.

VAP and/or ARDS was diagnosed in 38 patients (VAP/ARDS group: $29 \mathrm{VAP}$ and 9 ARDS), and the 20 others formed the control group. No significant difference was observed between the two groups for clinical parameters. There was no significant difference in the mean

\begin{tabular}{lccc} 
& $\begin{array}{c}\text { VAP/ARDS } \\
\text { group (range) }\end{array}$ & $\begin{array}{c}\text { control group } \\
\text { (range) }\end{array}$ & $P$ value \\
\hline neutrophils $\times 10^{6} / \mathrm{ml}$ & $1.21 \pm 0.35$ & $0.18 \pm 0.11$ & 0.0023 \\
$\mathrm{NTP} \mu \mathrm{g} / \mathrm{ml}$ & $13.50 \pm 3.80$ & $0.8 \pm 0.4$ & 0.025 \\
$\mathrm{MPO} \mathrm{U} / \mathrm{ml}$ & $1.66 \pm 0.45$ & $0.25 \pm 0.1$ & 0.0012 \\
elastase U/ml & $6.93 \pm 2.96$ & $0.008 \pm 0.003$ & 0.001 \\
$\mathrm{IC} \%$ & $51 \pm 15$ & $8.7 \pm 1.3$ & 0.01 \\
\hline
\end{tabular}

protein value of $B A L$ between the two groups $(1.49 \pm 0.17 \mathrm{mg} / \mathrm{ml}$ in VAP/ARDS versus $1.38 \pm 0.34$ in controls). On the contrary, a significant difference was found for the neutrophil count, NTP, myeloperoxidase and elastase values, and for cytotoxicity (IC: cytotoxicity index expressed in \%).

The cytotoxicity of BAL was correlated with the values of NTP $(r=0.92 ; P<0.001)$ and MPO ( $r=0.88 ; P<0.001)$. In $19 \mathrm{BAL}, \mathrm{NFKB}$ was found to be activated, and this activation was correlated with the BAL value of IL8 $(P<0.005)$.

From these data, we concluded that MPO and elastase were released in the alveoli by activated neutrophils and that NTP were formed in situ by the oxidant activity of stimulated neutrophils (in situ production of peroxynitrite and/or activity of MPO on nitrite or peroxynitrite). This intra-alveolar oxidant activity led to the production of $B A L$ fluids which were cytotoxic on alveolar cells and which enable the activation of the signal transduction pathway. However, the exact consequences of this NFKB activation and the particular compounds of BAL responsible for cytotoxicity remain to determine.

\section{P97 Cardiopulmonary effects of bronchoalveolar lavage in critically ill patients with ventilator- associated pneumonia}

\section{A Koroneos, I Kalomenidis, F Moraitou, P Polakis, S Papanikolaou and G Katsaris}

ICU, Nikaia-Piraeus General State Hospital, Nikaia, Fanarioton Street, Greece

Purpose: The purpose of this study was to evaluate the cardiopulmonary effects of bronchoalveolar lavage (BAL) in critically ill patients with ventilator-associated pneumonia (vap).
Method: Ten sedated and paralyzed critically ill patients suspected of having vap and requiring invasive hemodynamic monitoring were studied. A pulmonary artery catheter with continuous $\mathrm{SvO}_{2}$ and $\mathrm{CO}$ display was 
inserted to titrate administration of fluids and vasopressors. The $\mathrm{FiO}_{2}$ was adjusted to $1.0,30$ min before the performance of BAL for bacteriologic diagnosis and remained at this level for $6 \mathrm{~h}$ after the procedure. The following parameters were determined (1) before, (2) immediately after, (3) $2 \mathrm{~h}$ after and (4) $6 \mathrm{~h}$ after the BAL: compliance, $\mathrm{P}_{\text {peak }}$, $\mathrm{P}_{\text {plat }}, \mathrm{P}_{\mathrm{i}}, \mathrm{Rs}_{\mathrm{max}}, \mathrm{Rs}_{\min }, \Delta \mathrm{Rs}, \mathrm{PO}_{2}, \mathrm{PCO}_{2}, \mathrm{pH}, \mathrm{SaO}_{2}, \mathrm{SvO}_{2}$, $A P(S / D / M), P A P(S / D / M), C V P, P C W P, C l$, heart rate, PVRI, SVRI, LVSWI, RVSWI, $\mathrm{DO}_{2} \mathrm{l}, \mathrm{VO}_{2}, \mathrm{CcO}_{2}, \mathrm{CaO}_{2}$, $\mathrm{CvO}_{2}, \mathrm{C}(\mathrm{a}-\mathrm{v}) \mathrm{O}_{2}, \Delta \mathrm{PCO}_{2}, \mathrm{O}_{2} \mathrm{ER}, \mathrm{Q}_{\mathrm{s}} / \mathrm{O}_{\mathrm{t}}$.

The paired t-test method was used to compare the results at the four different time frames. Patients with necessary manipulation in the infusion rate of fluids-vasopressors and/or the parameters of mechanical ventilation during the $6 \mathrm{~h}$ period were excluded from data analysis.

Results: The results of eight patients were analyzed. Compliance showed after a nonsignificant fall from (1) to
(2), an increase from (2) to (3) $(P=0.038)$ and from (2) to (4) $(P=0.017) . P_{\text {plat }}$ increased slightly from (1) to (2) and decreased afterwards significantly from (2) to (4) $(P=0.017) . \mathrm{PCO}_{2}$ increased from (1) to (2) $(P=0.015)$ and remained high afterwards with parallel drop in $\mathrm{pH}$ from (1) to (2) $(P=0.006)$. The $\mathrm{Cl}$ showed, after a fall from (1) to (3) $(P=0.031)$, a gradual improvement. PVRI demonstrated, after a nonsignificant fall from (1) to (2), a gradual increase after (2) which was significant at (4) $(P=0.026)$. No further significant differences were recognized in every other parameter studied.

Conclusions: BAL in our patient population was a safe procedure. It did not affect oxygenation and pulmonary shunt. Compliance and $\mathrm{P}_{\text {plat }}$ showed after a nonsignificant initial deterioration gradual improvement. The delayed fall of $\mathrm{Cl}$ did not persist at the end of the study. The increase in PVRI needs further evaluation.

\section{P98 The influence of empiric antimicrobial therapy on acquired pulmonary infection in patients with a chest injury on ICU}

I Chytra, E Kasal, R Pradl, J Voborníková and R Sviták

Department of Anaesthesiology and Intensive Care, University Hospital, Alej svobody 80, 30460 Plzen, Czech Republic

Introduction: Infections acquired in the intensive care unit (ICU) commonly complicate the course of critical illness. The administration of empiric antibiotics was identified as an independent risk factor for the development of subsequent nosocomial infection [1]. The aim of our study was to evaluate the influence of empiric antimicrobial therapy on the acquired pulmonary infection in patients with a chest injury on interdisciplinary ICU.

Methods: We analyzed a prospectively-collected database of 64 patients with a chest injury admitted for at least four days to the interdisciplinary ICU of university hospital in 1999. Patients were retrospectively divided into a group receiving, from admission to the ICU, antimicrobial therapy (ATB group, $n=41$ ) and a group without antimicrobial therapy during first $48 \mathrm{~h}$ after admission (No ATB group, $\mathrm{n}=23$ ). The age, APACHE II, ISS, TRISS, length of ICU stay, duration of mechanical ventilation, rate of isolated chest injury and associated diagnoses (multiple injury, abdominal injury, head injury), ICU mortality, and rate of acquired pulmonary infection were evaluated. $\chi^{2}$ test, Mann-Whitney and unpaired t-test were used accordingly; $P<0.05$ was considered statistically significant.

Results: No differences in most of the parameters were found between groups, except for the length of ICU stay,
Table 1

\begin{tabular}{lccc}
\hline & $\begin{array}{c}\text { ATB } \\
(n=41)\end{array}$ & $\begin{array}{c}\text { No ATB } \\
(n=23)\end{array}$ & $P$ \\
\hline Admission APACHE II & $18.8 \pm 8.3$ & $17.6 \pm 10.4$ & NS \\
ISS (Injury Severity Score) & $27.9 \pm 10.1$ & $24.5 \pm 14.1$ & NS \\
Length of stay (days) & $12.4 \pm 9.6$ & $5.9 \pm 2.6$ & * \\
Mechanical ventilation (days) & $9.1 \pm 8.7$ & $3.5 \pm 3.6$ & * \\
$\begin{array}{l}\text { Number of acquired } \\
\text { pulmonary infection }\end{array}$ & $17(41.5 \%)$ & $4(17.4 \%)$ & $*$ \\
& & & \\
\hline
\end{tabular}

Data expressed as mean $\pm \mathrm{SD},{ }^{*}=P<0.05, \mathrm{NS}=$ non-significant.

duration of mechanical ventilation and the incidence of acquired pulmonary infection (Table 1).

Conclusions: Empiric antimicrobial therapy in patients with the chest injury on ICU may increase the rate of pulmonary infections, prolong duration of mechanical ventilation and extend the time of ICU stay. There is a need for controlled studies to define the role of antibiotics in empiric therapy in trauma patients.

\section{Reference:}

1. Kollef MH et al:: Chest, 1997, 112:666-675. 


\section{P99 Non invasive mechanical ventilation (NINMV) in cardiac surgery}

\section{Ferrante, L Bianciardi, E Conti, A Quacquarelli, V Praštalo and B Amari}

Department of Cardiac Anaesthesia and Intensive Care, Poliambulanza Hospital, Via Bissolati 57, 25124, Brescia, Italy

Objective: To evaluate the efficacy of NINMV in cardiac surgical patients. In order to verify if, beside already established advantages in critical care patients [1] (less intubation related complications, minor discomfort for patients and less need for sedation), NINMV can be useful in managing the weaning process in the postoperative cardiac surgical course.

Design: Retrospective survey. We used NINMV with two indications: (1) patients with unexpected postoperative respiratory failure requiring reintubation; (2) patients extubated early and with incomplete postoperative recovery of cardiorespiratory stability.

Setting: Five bed intensive care unit at a cardiothoracic surgery centre.

Patients: Twenty NINMV patients representing $4.1 \%$ of the 484 patients operated on from October 1998 to September 1999 in our centre. Urgent surgery occurred in $12 \%$ of cases, emergency surgery in $4.75 \%$ of cases. Overall in-hospital mortality was 14/484 (2.2\%).
Interventions: We use a nasal or facial mask (Respironics Inc., Murrysville, Pennsylvania, USA) connected to a Servo Ventilator 300 (Siemens-Elema, Sweden). Patients were ventilated by Pressure Support (10-15 $\left.\mathrm{cmH}_{2} \mathrm{O}\right)$ with PEEP $\left(5-8 \mathrm{cmH}_{2} \mathrm{O}\right)$. Intermittent periods of NINMV were alternated with periods of spontaneous breathing accordingly to patients needs. Sedation was obtained by Remifentanyl infusion in the range 0.03-0.07 $\mathrm{\gamma} \mathrm{kg}^{-1} \mathrm{~min}^{-1}$.

Measurements and main results: Twenty patients. were treated by NINMV (two with indication 1 and 18 patients. with indication 2). The two patients with indication 1 were successfully weaned and discharged. None of the other 484 patients in this series needed reintubation.

Table 1 characterises patients with indication 2 and summarises results.

Only 3 patients had to be reintubated $\left(n^{\circ} 3\right.$ for unexpected haematological complication, $n^{\circ} 8$ for psychotic disturbances, $n^{\circ} 18$ failed full haemodynamic recovery).

Table 1

\begin{tabular}{|c|c|c|c|c|c|}
\hline pt & age & Pathology & Haemodynamic support & NINMV days & Outcome \\
\hline 1 & 19 & Ascend. Aorta Replacement, AR,MR & high & 3 & W \\
\hline 2 & 81 & MR, Pulm. Hypert., postop renal failure & high & 1 & W \\
\hline 3 & 72 & CABG, postop acute leukaemia & high & 3 & $\mathrm{R}$ \\
\hline 4 & 64 & CABG, postop redo $\mathrm{CABG}$ & high & 2 & W \\
\hline 5 & 62 & CABG, postop PTCA & low & 2 & W \\
\hline 6 & 55 & AR, MR, Pulm Hypert. & high & 3 & W \\
\hline 7 & 74 & MR,CABG,cardiogenic shock & high & 5 & W \\
\hline 8 & 71 & AR,CABG, postop psycomot. agitation & low & 4 & $\mathrm{R}$ \\
\hline 9 & 60 & MR, Pulm Hypert & medium & 2 & W \\
\hline 10 & 77 & CABG, COPD & medium & 1 & W \\
\hline 11 & 61 & CABG, Pulm. Fibrosis & medium & 2 & W \\
\hline 12 & 75 & MR & medium & 2 & W \\
\hline 13 & 70 & CABG, obesity & low & 1 & W \\
\hline 14 & 71 & CABG,postop renal failure & high & 3 & W \\
\hline 15 & 65 & AR,pulm Hypert & high & 1 & W \\
\hline 16 & 77 & CABG & low & 1 & W \\
\hline 17 & 42 & $\mathrm{AR}, \mathrm{CABG}$ & medium & 2 & W \\
\hline 18 & 77 & CABG, Hypertrophic. Myocard. & medium & 8 & $\mathrm{R}$ \\
\hline
\end{tabular}

$\mathrm{AR}=$ aortic replacement, $\mathrm{MR}=$ mitral replacement, $\mathrm{CABG}=$ coronary artery bypass grafting, $\mathrm{COPD}=$ chronic obstructive pulmonary disease, PTCA=percutaneous coronary angioplasty, $W=$ weaned, $R=$ reintubated. 
Conclusions: NINMV patients with indication 2 were weaned from respiratory support earlier than usual during a complicated postoperative course. The good results of our series suggest that NINMV indication 2 can be viewed as a weaning model. The most severe forms of circulatory impairment and the non-collaborative patient seem to be, accordingly to literature, exclusion criteria. A wider study would better precise inclusion and exclusion criteria in cardiac surgical patients.

\section{Reference:}

1. Slutsky AS: ACCP consensus conference. Mechanical ventilation. Chest 1993, 104: 1833-1859.

\section{P100 An audit of unplanned extubations in a medical intensive care unit}

\section{A Johan}

Tan Tock Seng Hospital, 11 Jalan Tan Tock Seng, Singapore 308433, Republic of Singapore

Aim: To study the factors associated with and the outcome of unplanned extubations (UE) in an adult medical intensive care unit (MICU).

Methods: Prospective observational study of all patients admitted to an 8 bed university-affiliated MICU from January to December 1998. All patients requiring orotracheal intubation and mechanical ventilation were enrolled at time of the first UE. Information on demographics, disease severity, sedation, ventilator parameters, nursing factors and outcome was captured. Patients were followed up until in-hospital death or hospital discharge.

Results: 312 (57.5\%) of the 543 patients admitted over the 12 months required intubation and mechanical ventilation. There were $27(8.7 \%)$ events of UE. The mean age of all UE patients was $57.8 \pm 21.0$ years with a mean APACHE ॥ score of $18.8 \pm 9.8$. The mean number of days prior to UE, mean MICU length of stay (LOS) and mean hospital LOS were $3.2 \pm 3.3,9.5 \pm 10.4$ and $26.0 \pm 34.7$ respectively There were observed differences in MICU LOS (12.7 \pm 11.4 versus $3.2 \pm 2.6)$ and hospital LOS (31.4 \pm 41.2 versus 15.1 \pm 10.8 ) between the groups who did and did not require reintubation. All 6 deaths came from the group that was reintubated. These 6 patients were not weaning when UE occurred. $17(63 \%)$ of the UE events were deliberate despite $16(59 \%)$ and $20(74 \%)$ of this cohort being on chemical and physical restraints respectively. Nursing ratios of 1 nurse to 2 or 3 patients, depending on shift, were acceptable by our hospital standards and UE events occurred during period of decreased nursing acuity during night shift, change of shift and break time.

Conclusion: $8.7 \%$ UE, the majority being deliberate, was observed in our MICU. Patients who extubated during ventilator weaning had a better outcome. Windows of decreased nursing acuity may have contributed to the UE. Manpower redistribution during these windows, the adoption of improved sedation titration protocols and protocols to screen patients for readiness for extubation in our MICU may further reduce the risk for deliberate unplanned extubations.

\section{P101 Timing of extubation after esophagectomy}

\section{FHY Yap, ACW Chan and GM Joynt}

Department of Anaesthesia and Intensive Care, Prince of Wales Hospital, Shatin, HKSAR

Introduction: Prior to 1995 , it had been our standard practice to ventilate patients overnight after esophagectomy. Since then, however, this practice has changed. If patients are warm, hemodynamically stable, and comfortable, we have elected to extubate them early.

Aim: To study the association between time to extubation and outcome after esophagectomy.

Methods: We retrospectively reviewed the records of all esophagectomies between 1990 and 1998. Early extubation was defined as extubation within $12 \mathrm{~h}$. The primary outcomes evaluated were respiratory complications, hospital mortality and length of ICU stay. Outcomes between the two time periods 1990-94 (first period) and 1995-98 (second period) were compared. In the second time period, the relationship between time to extubation and outcomes was also evaluated.
Results: There were 78 esophagectomies in the first period (1990-94) and 84 in the second period (1995-98). In the second period, more patients received epidural analgesia $(64 \%$ vs $34 \%, P=0.001)$ and more patients were extubated early $(41 \%$ vs $6 \%, P<0.001)$ compared to the first period. There were no significant differences in the rates of respiratory complications ( $27 \%$ vs $34 \%$ ) and hospital mortality (6\% vs $10 \%)$ when comparing the second with the first time period.

In the second time period (1995-98), 31 of the 84 patients were extubated early (median time to early extubation was $6.8 \mathrm{~h}$ postop). More patients in the early extubation group received epidural analgesia ( $90 \%$ vs $50 \%, P=0.001$ ). There were again no significant differences in the rates of respiratory complications (29\% vs $30 \%)$ and hospital mortality $(6.7 \%$ vs $7 \%)$, but the late extubation group stayed longer in the ICU (median stay 2 days vs 1 day, $P=0.006$ ). 
Conclusions: Our experience suggests that early extubation after esophagectomy in stable patients is safe, and may decrease the length of ICU stay with cost-saving implications. We believe that thoracic epidural analgesia may facilitate early extubation by enabling patients to cough and cooperate with physiotherapy in the early postoperative period.

\section{P102 Evaluation of different CPAP systems in ICU population}

\section{Chiumello, P Pelosi, N Bottino, E Calvi, P Taccone and L Gattinoni}

Institute of Anaesthesia and ICU, Policlinico Hospital, IRCCS, University of Milan, Italy

The aim of this study was to evaluate the effects of different CPAP delivery systems in ICU patients. We tested four systems: 1) a home-made CPAP low flow circuit with an inspiratory reservoir and expiratory water valve (LW); 2) the same system with an expiratory mechanical valve (Vital Sign's) (LM); 3) a high flow CPAP system (Jet Venturi) with an expiratory mechanical valve (Vital Sign's) (HM); 4) the CPAP of the mechanical ventilator Servo 300 (Siemens); 5) the CPAP of Servo 300 plus three additional breaths per min delivered in pressure control ventilation (i.e. plateau airway pressure of $30 \mathrm{cmH}_{2} \mathrm{O}$ ) (ServoPC). In 13 intubated patients (9 males, PEEP level

of $6 \pm 2 \mathrm{cmH}_{2} \mathrm{O}$ and $\mathrm{PaO}_{2} / \mathrm{FiO}_{2}$ 250 \pm 10$)$ during the weaning phase we measured the breathing pattern, work of breathing, gas exchange and functional residual capacity. Data are expressed as mean \pm sd.

We found no differences between the low flow, either water or mechanical valve, and the high flow CPAP systems. The Servo 300 reduced the work of breathing, while the same CPAP system plus three additional breaths caused a further reduction in work of breathing and improvement in gas exchange and FRC.

\begin{tabular}{|c|c|c|c|c|c|}
\hline & LW & LM & $\mathrm{HM}$ & Servo & ServoPC \\
\hline $\mathrm{RR}$ bpm & $29 \pm 6$ & $29 \pm 7$ & $28 \pm 6$ & $28 \pm 6$ & $26 \pm 8$ \\
\hline TV ml & $401 \pm 138$ & $367 \pm 116$ & $393 \pm 147$ & $380 \pm 148$ & $336 \pm 171$ \\
\hline PTP $\mathrm{cmH}_{2} \mathrm{O}^{*} \mathrm{sec} / \mathrm{min}$ & $324 \pm 116$ & $303 \pm 124$ & $297 \pm 68$ & $240 \pm 96^{*}$ & $153 \pm 93^{\star}$ \\
\hline P. $01 \mathrm{cmH}_{2} \mathrm{O}$ & $-3.7 \pm 1.8$ & $-3.4 \pm 1.6$ & $-3.7 \pm 2.6$ & $-2.8 \pm 1.9$ & $-2.1 \pm 1.1$ \\
\hline $\mathrm{PaO}_{2} \mathrm{mmHg}$ & $98 \pm 18$ & $98 \pm 18$ & $103 \pm 21$ & $104 \pm 19$ & $125 \pm 23^{*}$ \\
\hline FRC L & $1.61 \pm 0.95$ & $1.59 \pm 0.95$ & $1.57 \pm 1.03$ & $1.68 \pm 0.88$ & $2.14 \pm 0.92^{*}$ \\
\hline
\end{tabular}

${ }^{*} P<0.05$ vs others.

P103 A comparison of the effects of conventional and balloon laryngoscopy on the sagittal dimensions of the space available for the cord at the level of the occipitoatlantoaxial complex

SD Mentzelopoulos, MJ Tzoufi and EP Papageorgiou

Department of Anesthesiology of Egion General Hospital, Egion, Greece

Background: The purpose of this study was to compare the sagittal surface areas (SSAs) of the space available for the cord (SAC) at the occiput (OCC)-first cervical vertebra (C1) and $\mathrm{C} 1-\mathrm{C} 2$ levels determined at neutral head position, and during conventional and balloon laryngoscopy $[1,2]$.

Methods: Anesthesia was induced in eight ASA I, Mallampati I, elective surgery patients. Cross-table lateralview radiographs were taken at neutral head position (NHP) and upon maximal laryngeal exposure (MLE) with a \#4 standard and a \#4 modified blade carrying two 10 Foley catheters. MLE during balloon laryngoscopy was achieved by right catheter-balloon inflation with $2 \mathrm{ml}$ air and blade-elevation $[1,2]$. The radiographs were scanned in Photoshop 5.0, the X-ray magnification was determined, and the OCC-C1 and C1-C2 SAC-SSAs were measured with Autocad 2000. The OCC-C1-SAC and C1-C2-SAC anatomic landmarks are shown in Fig. 1. Data were analyzed with Kolmogorov-Smirnov test and ANOVA.

Results: At the OCC-C1 level, the SAC-SSA was significantly reduced during conventional laryngoscopy (MLEvalue $=0.386 \pm 0.104 \mathrm{in}^{2}, \mathrm{NHP}$-value $=0.501 \pm 0.11 \mathrm{in}^{2}$, $P=0.0095)$. During balloon laryngoscopy, there was no significant SAC-SSA reduction (MLE-value $=0.423 \pm 0.128 \mathrm{in}^{2}$, $P=0.07$ vs NHP-value), while the SAC-SSA was significantly greater than the SAC-SSA determined during conventional laryngoscopy $(P=0.044)$. At the C1-C2 level, the SAC-SSA-changes were nonsignificant.

Conclusions: The OCC-C1-SAC is significantly less affected by balloon laryngoscopy. 
Figure 1

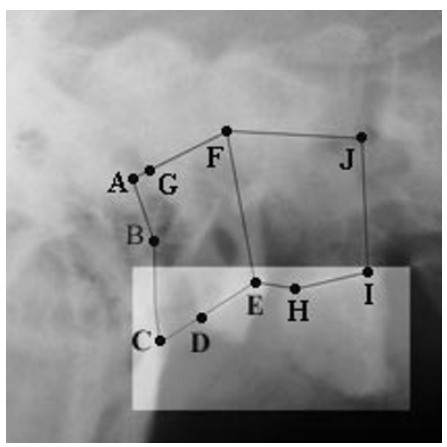

References:

1. Mentzelopoulos SD et al: Anesth Analg 1999, 88:1425-1426.

2. Mentzelopoulos SD et al: Crit Care 1999, 3(suppl 1):3-4.

$\mathrm{ABC}=$ inferior margin of occiput. $\mathrm{DE}=\mathrm{C} 1$-spinous process base. $\mathrm{E}=$ most caudal point of $\mathrm{C} 1$-spinous process base. $\mathrm{F}=$ radiographic intersection of $\mathrm{C} 1$ by odontoid-posterior cortex. $\mathrm{G}=$ most cephalad point of odontoid-posterior cortex. $\mathrm{HI}=\mathrm{C} 2$-spinous process base. $\mathrm{l}=$ most caudal point of $\mathrm{C} 1$-spinous process base. $\mathrm{FJ}=$ posterior cortex of C2-vertebral body

\section{P104 Use of Combitube airway to protect the airway from methylene blue dye}

\section{MH Mercer}

Department of Anaesthesia, Frenchay Hospital, Bristol BS16 1LE, United Kingdom

Introduction: The Combitube airway (Kendall UK Ltd., Basingstoke, England) is included in the European Resuscitation guidelines for the management of the emergency airway [1]. In the trauma patient tracheal soiling is usually from the upper airway [2]. Although the proximal, large cuff of the Combitube may protect the airway, this has not been formally assessed.

Method: After local ethics committee approval, 10 ASA 1 and 2, starved patients undergoing routine elective general anaesthesia involving non-depolarising neuromuscular blockade and mechanical ventilation of the lungs gave informed consent. Only patients with grade 1 direct laryngoscopic views were included to ensure adequate views of the vocal cords. The small adult sized Combitube was placed into the oesophagus, all patients being of the appropriate height, and the cuffs inflated. $10 \mathrm{ml}$ of $0.1 \%$ methylene blue dye was instilled into the mouth. Full monitoring was used, and adequate ventilation ensured throughout. At the end of surgery all dye was suctioned away and the oral cavity and proximal Combitube cuff manually cleaned with absorbent gauze. The Combitube was removed and the airway examined laryngoscopically.

Results: One patient had an initial airway leak that settled with minor Combitube realignment prior to dye instillation. No laryngeal dye was detected in 9 patients. One patient had significant blue staining of the vocal cords.

Conclusion: The Combitube therefore protects the majority of patients airways from aspiration of dye from the oral cavity. In the trauma setting blood and oral debris may therefore be prevented from entering the trachea. The one failure was probably a result of patient movement by surgeons as this same patient developed a ventilatory leak mid-operation. However, patient movement would be expected in the trauma population.

\section{References:}

1. European Resuscitation Council: Resuscitation 1996, 31:201-230.

2. Lockey DJ, Coats T, Parr MJA: Aspiration in severe trauma: a prospective study. Anaesthesia 1999, 54:1097-1098.

\section{P105 Translaryngeal tracheostomy: prospective experience in two Canadian tertiary intensive care units}

\section{Sharpe ${ }^{*}$, L Parnes ${ }^{\dagger}, C_{\text {Harris }}^{*}$ and J Drover ${ }^{\ddagger}$}

${ }^{*}$ Departments of Anaesthesia and ${ }^{\dagger}$ Otolaryngology, London Health Sciences Centre, 339 Windermere Road, London, Ontario

Canada N6A 5A5; ₹Program in Critical Care Medicine, University of Western Ontario, and Department of Surgery, Kingston

General Hospital, Queen's University, Kingston, Ontario, Canada

Introduction: Bedside percutaneous tracheostomy has supplanted open surgical tracheostomy as the procedure of choice in many Intensive Care Units for patients who require prolonged mechanical ventilatory support. The new translaryngeal tracheostomy (TLT) method described by Fantoni et al. [1] has inherent advantages over other percutaneous techniques. We present our prospective review of 111 cases using this new technique. 
Methods: During a 26 month period, a TLT was performed on 111 patients who required an elective tracheostomy in our two ICUs. Under general anesthesia, the TLT was performed at the bedside using the Translaryngeal Tracheostomy kit (Mallinckrodt, Italy). This technique is performed under direct visualization with either a flexible bronchoscope or a rigid $30^{\circ}$ endoscope. The tracheostomy tube with its dilator is pulled from within the trachea using a guide wire that is initially placed during direct visualization. After rotating the cut tube into place, bronchoscopic visualization confirms its correct position within the trachea.

Results: All 111 tracheostomies were performed successfully. Mean duration of the procedure was $28 \pm 11 \mathrm{~min}$ with a mean oxygen saturation of $98 \pm 3 \%$; the lowest saturation occurring was $81 \%$. Desaturations occurred in 20 patients; however, they were short lived and caused no harm. Mean blood pressure was $77 \pm 26 \mathrm{mmHg}$. Blood loss was minimal $(<5 \mathrm{mls})$ in all cases. Mean platelet count was $277 \times 106 \pm 135$; range $39-818)$. Mean INR was 1.2 \pm 0.3 ; range 0.9-2.8. Mean PTT was $48 \pm 58$ s; range 22-554. All patients tolerated the procedure well. Postoperative wound infections did not occur. One patient was noted to have a pneumothorax the day following the procedure while on positive pressure ventilation.

Conclusion: This technique induces minimal trauma due to its 'dilation' of tissue as it passes through the anterior wall of the trachea. This new dilatational technique is easy, safe and reliable. Bleeding is minimal, even in patients with coagulopathies due to the tight approximation of the tube to the tissue. Ventilation and protection of the airway is maintained throughout the procedure. It has become our procedure of choice for patients requiring an elective tracheostomy.

\section{Reference:}

1. Fantoni et al:: Int Care Med 1997, 23:386-392.

\section{P106 The use of percutaneous tracheostomy in patients with severe acute or chronic liver disease}

\section{N Parnell, W Bernal and J Wendon}

The Institute of Liver studies, King's College Hospital, Denmark Hill, London, UK

Introduction: Percutaneous tracheostomy (PT) is recognised as a safe and effective method for prolonged ventilatory support, avoiding the complications of lengthy endotracheal intubation. Bleeding has been reported as the most common perioperative complication in most series [1]. Patients with severe liver disease frequently have a coagulopathy and thrombocytopaenia. We wished to assess the safety of PT in this group of patients, who are at high risk of haemorrhagic complications.

Methods: A retrospective study of PT's performed in patients admitted to a dedicated liver intensive care unit in a supraregional liver centre over two years. All procedures followed the Ciaglia technique [2] and were performed by, or under the supervision of, experienced operators. All patients had liver disease, which included decompensated chronic disease, hyperacute and acute liver failure and liver transplantation with severe postoperative complications.

Patients considered to be at particularly high risk for bleeding, based on platelet count (PIts) and international normalised ratio (INR) received transfusions of platelets and/or fresh frozen plasma prior to the procedure.
Results: The median age was 46.5 years with 20 males and 14 females. Five of the thirty-four patients suffered complications, none of which were fatal, and included minor bleeding only (requiring no treatment). The MannWhitney $U$ test was used to compare differences in INR and Plts between those with and without complications. The median pre-correction INR for the group without complications was $1.1(0.82-1.79)$ and for those with complications was $1.34(0.92-1.76)$ and this did not reach statistical significance. There was a trend towards lower Plts in those with a complication (median 44; range 21-84) compared to those without (median 76; range $3-510)$ but again this was not statistically significant $(P<0.3)$.

Conclusions: Although this is a small study, based on the data presented, PT appears to be a safe procedure in this group of patients, at high risk of bleeding, when standard precautions are followed.

\section{References:}

1. Petros S: Percutaneous tracheostomy. Crit care 1999, 3:R5-R10.

2. Ciaglia $P$, Firsching $R$, Syniec C: Elective percutaneous dilatational tracheostomy. Chest 1985, 87:715-719. 


\section{P107 In vitro study of a new vaporization humidifying device: DAR HC $2000^{\circledR}$}

\section{N Bottino, L Chiaravalli, M Panigada, F Carrieri, E Allegritti, P Pelosi ${ }^{*}$ and L Gattinoni}

1st. Anestesia e Rianimazione, Osp. Maggiore Policlinico-IRCCS, via F. Sforza, 35, 20122, Milan, Italy; *Dip. di Scienze Cliniche e Biologiche, Univ. dell'Insubria, Varese, Italy

In long-term mechanical ventilation, inspired gas should reach the endotracheal tube with a temperature of $30-32^{\circ} \mathrm{C}$, and an absolute humidity $\left(\mathrm{H}_{\mathrm{A}}\right)$ of approximately 28-30 $\mathrm{mg} \mathrm{H}_{2} \mathrm{O} / \mathrm{l}$.

We tested a humidifier (DAR HC 2000 ${ }^{\circledR}$ ) composed of: 1 ) a vaporising cartridge, containing a hydrophobic membrane that separates the gas flow from the heated water; 2) a heating wire that runs all along the inspiratory line, maintaining the heat and moisture conditions of the inspired gas from the cartridge to the patient; 3 ) a double temperature control that permits the setting of the desired heating level both at the cartridge $\left(\mathrm{T}^{\circ} \mathrm{C}\right)$ and at the patient's mouth $\left(T^{\circ} \mathrm{p}\right)$.

At first, we studied the system's ability to condition inspired gas at different min ventilation $\left(V_{E}=6,10,14\right.$ $\mathrm{l} / \mathrm{min})$, tidal volume $\left(\mathrm{V}_{\mathrm{T}}=250,500,750 \mathrm{ml}\right)$, and inspiratory flow $\left(\mathrm{V}_{\mathrm{i}}=0.5,1 \mathrm{l} / \mathrm{sec}\right)$ with different settings of $\mathrm{T}^{\circ} \mathrm{C}$ and $\mathrm{T}^{\circ} \mathrm{p}$ (32-32, 34-34, 36-36, 34-32, 36-32, 36-34, 38-34, $39-34^{\circ} \mathrm{C}$, respectively). We found that increasing $V_{E}$ from 6 to 10 to $14 \mathrm{l} / \mathrm{min}$ led to a progressive decrease of $\mathrm{H}_{\mathrm{A}}$ from $33.0 \pm 4.4$ to $27.5 \pm 4.4$ to $23.4 \pm 3.6 \mathrm{mg} / \mathrm{l}(P<0.01)$, while $V_{T}$ and $V_{i}$ had no effects on humidification.
Secondly, we tested the humidifier at different $V_{E}$ (from 4 to $16 \mathrm{l} / \mathrm{min}, 2 \mathrm{l} / \mathrm{min}$ per step) with different $\mathrm{T}^{\circ} \mathrm{p}(32,34$, $36^{\circ} \mathrm{C} ; \mathrm{T}^{\circ} \mathrm{C}$ initially set at the same level as $\mathrm{T}^{\circ} \mathrm{p}$, and then increased at $1^{\circ} \mathrm{C}$ at a time until condensate appeared in the thermoregulated tube). Adequate humidification levels $\left(H_{A}=30 \mathrm{mg} / \mathrm{l}\right)$ can be obtained for each $\mathrm{V}_{E}$ and $T^{\circ} \mathrm{p}$ by increasing the temperature set at the cartridge enough.

The third part of the study was to verify the decrease of microbiological contamination by simulating a contaminated patient (using a reservoir containing Micrococcus luteus) and analysing the inspiratory line with the heating wire switched first OFF, then ON: keeping the thermoregulated tube OFF, as supposed, a bacterial growth was seen in the inspiratory line, while switching it $\mathrm{ON}$, no $M$. luteus colonies were observable.

In conclusion, DAR HC $2000^{\circledR}$ humidifier allows us to obtain adequate levels of humidity of inspired gas for each ventilatory set, just by increasing the cartridge temperature. Besides, the hydrophobic membrane and the heating wire avoid, respectively, the gas-water contact and the condensate formation in the inspiratory line resulting in a reduction of microbiological contamination of the circuit.

\section{P108 Non-invasive two-point estimation of arterial $\mathrm{PCO}_{2}$ and alveolar deadspace}

\section{E Michel ${ }^{*}$ and JA Peper ${ }^{* \dagger}$}

${ }^{*}$ Emma Children's Hospital, PICU, and 'Department of Anaesthesiology, Academic Medical Centre, University of Amsterdam, Meibergdreef 9, NL-1105 AZ Amsterdam, The Netherlands

Introduction: Often end-tidal $\mathrm{PCO}_{2}\left(\right.$ etPCO $\mathrm{PC}_{2}$ gives a good estimate of arterial $\mathrm{PCO}_{2}\left(\mathrm{PaCO}_{2}\right)$. However, there are conditions, namely diseases with pulmonary ventilation/perfusion mismatch, where $\mathrm{PaCO}_{2}$ is severely underestimated.

Objective: We aimed to develop a method for the accurate non-invasive estimation of $\mathrm{PaCO}_{2}$ from etPCO $\mathrm{PC}_{2}$.

Method: The method is based on the model assumption that etPCO $\mathrm{P}_{2}$ is a mixture of $\mathrm{PCO}_{2}$ from both the ventilated and perfused alveoli, and the ventilated but non-perfused ones ('alveolar deadspace'). Knowing the etPCO $\mathrm{CO}_{2}$ under conditions of inspiratory gases with different $\mathrm{CO}_{2}$ content $\left(\mathrm{PCO}_{2} \mathrm{a} ; \mathrm{PCO}_{2} \mathrm{~b}\right)$, it is possible to calculate the $\mathrm{PCO}_{2}$ of the ventilated and perfused alveoli $\left(\mathrm{PCO}_{2} \mathrm{alv}\right)$. Knowledge of $\mathrm{PCO}_{2}$ alv allows to calculate the fraction of alveolar deadspace ventilation. As long as para-alveolar (i.e. cardiac) shunting is less than $20 \%, \mathrm{PCO}_{2}$ alv accurately reflects $\mathrm{PaCO}_{2}$.

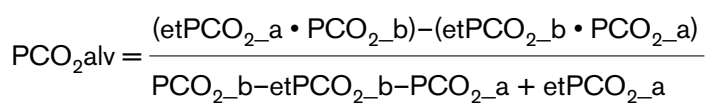

The model was applied on ventilated adult patients while inpiring different levels of $\mathrm{PCO}_{2}$. Estimates of $\mathrm{PaCO}_{2}$ and alveolar deadspace were compared to the results of invasive determination.

Results: Preliminary results indicate a good correlation between invasively and non-invasively determined $\mathrm{PaCO}_{2}$ and alveolar deadspace (Table 1).

Conclusions: Using gas void of $\mathrm{CO}_{2}$ as one of the two inspiratory gases further simplifies the formula. Our preliminary findings show that a difference in the levels of inspired $\mathrm{PCO}_{2}$ of at least $3 \mathrm{kPa}$ gives an estimate of $\mathrm{PaCO}_{2}$ with an accuracy of $10 \%$ or better. We provide a non-invasive method for the accurate estimation of $\mathrm{PaCO}_{2}$ 


\begin{tabular}{|c|c|c|c|c|c|c|c|c|c|}
\hline \multirow[b]{2}{*}{ No. } & \multirow{2}{*}{$\begin{array}{c}\mathrm{PCO}_{22} \mathrm{a} \\
(\mathrm{kPa})\end{array}$} & \multirow{2}{*}{$\begin{array}{l}\operatorname{etPCO}_{22} \mathrm{a} \\
(\mathrm{kPa})^{-1}\end{array}$} & \multirow{2}{*}{$\underset{(\mathrm{kPa})}{\mathrm{PCO}_{2-} \mathrm{b}}$} & \multirow{2}{*}{$\begin{array}{l}\text { etPCO }{ }_{2} b \\
(\mathrm{kPa})^{-b}\end{array}$} & \multicolumn{2}{|c|}{$\mathrm{PaCO}_{2}(\mathrm{kPa})$} & \multirow[b]{2}{*}{ difference (\%) } & \multicolumn{2}{|c|}{$\mathrm{Vd} / \mathrm{Vt}$} \\
\hline & & & & & calc. & measured & & calc. & measured \\
\hline 1 & 0 & 5.3 & 2.9 & 6.0 & 6.97 & 7.10 & -1.8 & 0.32 & 0.34 \\
\hline 2 & 0 & 5.3 & 5.0 & 6.6 & 7.16 & 7.10 & +0.9 & 0.35 & 0.34 \\
\hline 3 & 0 & 5.5 & 4.0 & 5.2 & 5.12 & 5.61 & -8.8 & -0.07 & 0.02 \\
\hline 4 & 0 & 5.5 & 4.4 & 5.4 & 5.38 & 5.61 & -4.1 & -0.02 & 0.02 \\
\hline 5 & 0 & 5.2 & 2.0 & 5.6 & 6.50 & 6.89 & -5.7 & 0.25 & 0.33 \\
\hline 6 & 0 & 4.1 & 1.3 & 4.4 & 5.36 & 5.98 & -10.4 & 0.31 & 0.46 \\
\hline 7 & 0 & 4.1 & 3.2 & 4.9 & 5.51 & 5.98 & -7.8 & 0.34 & 0.46 \\
\hline 8 & 0 & 4.1 & 4.4 & 5.6 & 6.22 & 5.98 & +4.0 & 0.52 & 0.46 \\
\hline
\end{tabular}

and alveolar deadspace ventilation. We suggest its implementation in ventilators for the close monitoring of pulmonary treatment response.

\section{P109 Hemodynamic effects of the inspiratory flow rate in patients with septic shock}

\section{A Koroneos, J Kalomenidis, F Moraitou, P Polakis, G Leptidis, V Kekeris and G Katsaris}

ICU, Nikaia-Piraeus General State Hospital, Fanarioton Street, Nikaia, Greece

Purpose: The purpose of the study was to assess the effects of changes in inspiratory flow (IF) on hemodynamic parameters of mechanically-ventilated patients with septic shock.

Method: Eight patients with septic shock (pneumonia $n=2$ and abdominal sepsis $n=6$ ) were included in the study. A pulmonary artery catheter with continuous $\mathrm{SvO}_{2}$ and $\mathrm{CO}$ display had been inserted to titrate fluid and vasopressor therapy. The patients were on volume control ventilation with a square flow waveform. Three different levels of IF were applied, each for a period of thirty $\min \left(\mathrm{IF}_{1}=40 \mathrm{I} / \mathrm{min}, \mathrm{IF}_{2}=50 \mathrm{I} / \mathrm{min}, \mathrm{IF}_{3}=60 \mathrm{I} / \mathrm{min}\right)$. At the end of each period the following parameters were recorded: CVP, PCWP, MAP, MPAP, CI, SVRI, PVRI,
LVSWI, RVSWI, $\mathrm{SaO}_{2}, \mathrm{SvO}_{2}, \mathrm{DO}_{2}$. The paired t-test method was used to compare the results at the three different levels of IF.

Results: Two patients were withdrawn from the study, due to necessary manipulation in the infusion rate of fluids and/or vasopressors. In six patients, a significant increase in LVSWI from $\mathrm{IF}_{1}$ to $\mathrm{IF}_{3}\left(5.22 \pm 1.38 \mathrm{~g} \cdot \mathrm{m} / \mathrm{m}^{2}\right.$ [mean $\pm S E$ ], $P=0.013)$ was observed. No further significant differences were recognized.

Conclusion: The change in the IF from 40 to $60 \mathrm{l} / \mathrm{min}$ did not have any negative effect in the hemodynamic profile of patients with septic shock. On the contrary, it led to an increase of the LVSWI.

\section{P110 Automatic tube compensation combined with pressure support ventilation - improved work of breathing pattern and less work}

\section{P Singer, J Cohen, A Abraham and E Grozovsky}

General Intensive Care Unit, Rabin Medical Center, Beilinson Campus, Petah Tikwa 49100, Israel

Introduction: Liberation from mechanical ventilation may be difficult due to resistive properties of the endotracheal tube (ETT), which increases work of breathing. PSV alone may not overcome this problem as the increased resistance largely limits expiratory gas flow-inspiration and expiration. The benefits of combining ATC with PSV have not been assessed.
Method: We included 34 patients ventilated with three levels of PSV $\left(<15,15-20,20-30 \mathrm{CMH}_{2} \mathrm{O}\right)$. ATC with $100 \%$ conpensation was then added for 30 min, stopped for $30 \mathrm{~min}$ and re-instituted for $30 \mathrm{~min}$. At the end of each period we measured respiratory rate (RR), tidal volume (TV), minute ventilation (MV), PEEPi, HR, VO, $\mathrm{VCO}_{2}$ and energy expenditure (EE). 
Results are given as median and $\pm \mathrm{SD}$

\begin{tabular}{|c|c|c|c|c|c|}
\hline Parameters & With ATC & Without ATC & $P$ & With ATC & $P$ \\
\hline $\mathrm{RR} / \mathrm{mnt}$ & $25 \pm 7.6$ & $31 \pm 7.3$ & 0.01 & $23 \pm 6.9$ & 0.04 \\
\hline TV (ml) & $513 \pm 20.4$ & $472 \pm 13.5$ & 0.02 & $324 \pm 125.5$ & 0.03 \\
\hline MV (L/mnt) & $11 \pm 3.7$ & $12.3 \pm 3.55$ & 0.01 & $11 \pm 3.5$ & 0.03 \\
\hline PEEP $i$ & $5.4 \pm 2.5$ & $7.4 \pm 2.6$ & 0.01 & $4.7 \pm 2.7$ & 0.07 \\
\hline $\mathrm{HR} / \mathrm{mnt}$ & $97 \pm 29.4$ & $107 \pm 16$ & NS & $96 \pm 21$ & 0.09 \\
\hline V02 & $332 \pm 65.9$ & $343 \pm 65.9$ & 0.02 & $344 \pm 64.9$ & NS \\
\hline VCO2 & $295 \pm 63.9$ & $293 \pm 3.9$ & NS & $296 \pm 61.3$ & NS \\
\hline EE (Kcal/D) & $2320 \pm 42.8$ & $2360 \pm 442.8$ & $0 / 01$ & $2390 \pm 460.9$ & NS \\
\hline
\end{tabular}

No significant differences were found for the parameters studied at other levels of PSV.
Conclusions: The addition of ATC to low levels of PSV $(<15 \mathrm{CM}$ of water) results in improved breathing patterns with metabolic benefits to the patient.

\section{P111 Proportional pressure support in acute lung injury: an observational study}

\section{Capra and S Manera}

Intensive Care Unit, Saronno, P.zza Borella 1, Saronno (VA), Italy

Proportional Pressure Support (PPS ${ }^{\mathrm{Tm}}$ ) is a partial ventilatory support that overcomes patient respiratory system elastance (Ers) and resistance (Rrs) by applying pressure 'proportional' to volume (volume assist, VA) and flow (flow assist, FA) respectively. The aim of this study was to evaluate its clinical use at the bedside in critically ill intubated patients affected by acute lung injury (ALI).

Methods: Thirty previously healthy patients affected by post-traumatic (emopneumothorax and lung contusion, $\mathrm{N}=21$ ), infective (pneumonia, $\mathrm{N}=8$ ) and inflammatory (pancreatitis, $\mathrm{N}=1$ ) ALI (American-European Consensus Conference on ARDS) were studied. After several days (range 2-7, mean 4) of assist/controlled (A/C) ventilation $\left(\mathrm{BIPAP}^{\mathrm{TM}}\right)$, patients were switched to PPS once they were stable. Ers and Rrs were determined during a short period of volume control ventilation (square flow wave, tidal volume $10 \mathrm{ml} / \mathrm{kg}$ ) using the inspiratory hold technique. On the ventilator (EVITA 4, Draeger) FA and VA were set to $80 \%$ value of patient Ers and Rrs respectively. The Automatic Tube Compensation (ATC ${ }^{\mathrm{m}}$ ) was also used at $100 \%$ compensation. $\mathrm{O}_{2}$ inspiratory fraction $\left(\mathrm{FiO}_{2}\right)$ and positive end-respiratory pressure (PEEP) were the same as in $A / C$ mode. Progressive reduction of FA, VA, PEEP and $\mathrm{FiO}_{2}$ was used as the weaning technique, during the healing phase of the disease. The following parameters were ascertained daily: arterial blood gas analysis, respiratory rate $(\mathrm{RR})$, tidal volume $(\mathrm{Vt})$, minute ventilation $(\mathrm{Ve})$, patients' comfort, number of respiratory distress that needed changes in ventilatory mode and failure to wean. The FA and VA values at which the patients were able to sustain spontaneous breathing and extubation were registered.
Results: $\mathrm{PaO}_{2} / \mathrm{FiO}_{2}$ ratio remained unchanged or slightly better compared to $\mathrm{A} / \mathrm{C}$ mode, and progressively returned to normal during recovery. $\mathrm{PaCO}_{2}$ ranged between normal values and $\mathrm{pH}$ ranged between 7.45 and 7.40 for each patient during the study period. The respiratory pattern changed greatly in terms of RR and Vt from one patient to another and in the same patient during the days, while Ve remained more stable. All patients, except two, were always comfortable and they were successfully weaned and extubated at mean values of $\mathrm{FA}=7 \pm 2 \mathrm{cmH}_{2} \mathrm{O} / \mathrm{l} / \mathrm{sec}$ and $\mathrm{VA}=8 \pm 3 \mathrm{cmH}_{2} \mathrm{O} / \mathrm{l}$. The two patients that experienced respiratory distress were affected by pneumonia and needed a change of ventilatory mode.

Conclusion: PPS ${ }^{\mathrm{TM}}$ with $\mathrm{ATC}^{\mathrm{TM}}$ may be used to assist the ventilatory needs of patients with post-traumatic, infective or inflammatory ALI. The clinician must not be frightened by an unusual respiratory pattern in the presence of a comfortable patient, because with PPS ${ }^{\text {TM }}$ patients are able to breathe as they like to maintain a normal $\mathrm{pH}$, most likely because the neuroventilatory coupling is improved by this mode, as described by Younes [1]. Extensive studies are needed to evaluate the role of PPS ${ }^{\text {TM }}$ with ATC $^{\text {TM }}$ among the ventilatory modes now available to support the patient with ALI.

\section{Reference:}

1. Younes M: Proportional Assist Ventilation. In Principles and Practice of Mechanical Ventilation. Tobin MJ: Mc Graw-Hill, Inc.; 1994:349-396. 
http://ccforum.com/supplements/4/S1

\section{P112 Selected biochemical values and organ dysfunction assessment in prediction of difficult to wean patients}

V Cerny, P Zivny*, P Dostal and R Parizkova

Department of Anesthesiology and Intensive Care, *Department of Biochemistry, Charles University, Faculty of Medicine, 50005 Hradec Králové, Czech Republic

Introduction: Metabolic status and severity of illness play an important role in developing ventilatory dependency during long term ventilatory support in critically ill patients. The aim of the study was to evaluate selected clinical and biochemical values in order to identify 'difficult to wean' and 'easy to wean' patients during ventilatory support.

Methods: After institutional approval, 40 critically ill patients were prospectively studied during ventilatory support and weaning. All patients were weaned according to standard weaning protocol. Blood samples were drawn daily and collected until analysis. APACHE II score, organ failure score (Goris), sepis organ failure assessment score (SOFA), ventilatory days, and 'weaning' days were recorded. After successful weaning, patients were divided into two groups according to the length of weaning (W): group $S(W \leq 3$ days, $n=15)$, group $L(W>3$ days, $n=22)$. Serum levels of albumin, prealbumin, cholinesterase, CRP, magnesium, phosphorus and urine levels of myoglobin were measured during ventilatory support (including weaning trials). SigmaStat Statistical Software was used for analysis, $\mathrm{r}=$ corr. coeff., ${ }^{\star} P<0.05$.

Results: There were no significant differences in any biochemical values between groups during ventilatory support. In group $L$ there were positive significant correlations between SOFA score and length of ventilatory support and weaning $(r=0.473, P=0.035)$ and between Goris score and length of ventilatory support $(r=0.626$, $P=0.003$ ).

Conclusion: Assesment of severity of organ dysfunction, but no special biochemical values, seems to be from 'cost/effectiveness point-of-view' the best way to identify patients with need for long term ventilatory support and who are difficult to wean.

\section{P113 Respiratory mechanics studied by multiple regression and the end-inspiratory pause technique during mechanical ventilation}

MP Vassiliou, Ch Psarakis, A Amygdalou, K Kalopissi, M Moukas and PK Behrakis

Pulmonary Function Laboratory, 'St. Savas' Hospital and Laboratory of Experimental Physiology, Medical School, University of Athens, Athens, Greece

Aim of the study: In the present study, a comparative evaluation of the multiple linear regression analysis (MLRA) and the end-inspiratory pause technique (EIP) is attempted for the investigation of respiratory system (RS) mechanics during mechanical ventilation.

Methods: Airway pressure (Pao) and flow $\left(\mathrm{V}^{\prime}\right)$ data were digitally obtained from 25 ICU patients, who were mechanically ventilated (CMV mode) and under sedation and muscle relaxation. Volume (V) was calculated by numerical integration of $\mathrm{V}^{\prime}$. Data were analysed on a cycle per cycle basis with the aid of MLRA according to: $\mathrm{Pao}=\mathrm{PE}+$ Ers.V + Rrs.V', where Ers represents the RS Elastance, Rrs the RS resistance and PE the end-expiratory pressure. Ers and Rrs were also calculated with the aid of the EIP, while end-expiratory pressure was measured on the actual Pao signal. Ers(EIP), Rrs(EIP) and $P E(E I P)$ were used to reconstruct the pressure, according to EIP. Predicted Pao according to MLRA and EIP were correlated to the measured Pao. The error of correlations measured as the root mean square difference (RMSD) was used for the comparative evaluation of the two techniques. Student paired t-test was used for the comparison of the calculated mechanical coefficients $(P=0.05)$.

Results: Results are presented in the table (*denotes statistically significant difference at $P=0.05$ ).

\begin{tabular}{lcccc} 
& $\operatorname{Ers}(\mathrm{hPa} / \mathrm{L})$ & $\operatorname{Rrs}(\mathrm{hPa} / \mathrm{L} / \mathrm{s})$ & $\mathrm{PE}(\mathrm{hPa})$ & $\mathrm{RMSD}(\mathrm{hPa})^{*}$ \\
\hline MLRA & $29.15 \pm 8.832$ & $12.42 \pm 4.346$ & $2.10 \pm 2.466$ & $1.17 \pm 0.590$ \\
EIP & $28.06 \pm 8.314$ & $12.48 \pm 4.157$ & $1.86 \pm 1.746$ & $1.40 \pm 0.738$ \\
\hline
\end{tabular}

Conclusions: Both MLRA and EIP offer an almost equal and linear approach to respiratory mechanics during mechanical ventilation. MLRA gives more accurate results according to the better correlation (lower RMSD) of MLRA pressure than EIP pressure to the actually measured Pao. 


\title{
P114 Open lung strategies in patients with intracranial lesions and acute lung injury
}

\section{S Wolf, L Schürer, HA Trost and Ch B Lumenta}

Department of Neurosurgery, Academic Hospital Munich-Bogenhausen, Englschalkinger Strasse 77, 81925 Munich, Germany

Objective: For patients with acute lung injury, a recent randomized controlled trial showed the superiority of a ventilator strategy with low tidal volumes and elevated PEEP levels after performing recruiting maneuvers (together termed the 'open lung approach') compared to a conventional approach with tidal volumes of $12 \mathrm{ml} / \mathrm{kg}$ body weight [1]. However, patients with intracranial lesions were excluded from this and other ARDS studies due to the potential risk of exacerbation of intracranial pressure.

Methods: We present the clinical course of eight patients with known intracranial pathology and concomitant acute lung injury, which was treated according to the open lung concept. Primary diagnosis was either traumatic brain injury $(n=3)$, subarachnoid hemorrhage $(n=2)$, cerebellar hemorrhage $(n=1)$, cerebellar infarction $(n=1)$ or frontal meningeoma $(n=1)$. ICP monitoring was present in all patients, brain tissue oxygen $\left(\mathrm{p}_{\mathrm{ti}} \mathrm{O}_{2}\right)$ monitoring in two of them. Data was collected with multimodal monitoring devices with a sampling rate of 1 per min.

Results: Ventilation according to the open lung approach improved the mean oxygenation index $\left(\mathrm{p}_{\mathrm{a}} \mathrm{O}_{2} / \mathrm{F}_{\mathrm{i}} \mathrm{O}_{2}\right)$ signifi- cantly from $130 \pm 104$ before to $344 \pm 63$ measured $24 \mathrm{~h}$ after first recruiting $(P<0.001)$. Mean PEEP after the first recruitment maneuver was $14.9 \pm 2.7 \mathrm{mmHg}$. ICP values during the first $24 \mathrm{~h}$ rose slightly (not significant) in seven of the eight patients, requiring standard treatment with immediate response in two of them. In one patient, the ICP decreased from $33 \mathrm{mmHg}$ to $25 \mathrm{mmHg}$ after the first recruitment maneuver. Relevant change of $\mathrm{p}_{\mathrm{ti}} \mathrm{O}_{2}$ values was not noticed, as the $\mathrm{F}_{\mathrm{i}} \mathrm{O}_{2}$ was consequently lowered, according to provide an arterial $\mathrm{p}_{\mathrm{a}} \mathrm{O}_{2}$ around $100 \mathrm{mmHg}$, and with respect of a $\mathrm{p}_{\mathrm{ti}} \mathrm{O}_{2}$ above $20 \mathrm{mmHg}$.

Conclusion: Although this study was done as a feasibility analysis and lacks a control group, our results encourage us to propose open lung strategies, which had been restricted up to now, for neurosurgical patients with acute lung injury. The potential risk of intracranial deterioration due to critical ICP values seems far lower than the achievable benefit and decrease of mortality due to improved oxygenation.

\section{Reference:}

1. Amato MB et al.: N Engl J Med 1998, 338:347-354.

\section{P115 Relationship between superimposed pressure and pleural pressure gradient in an experimental model of ARDS}

\author{
N Bottino, G Eccher, P Pelosi ${ }^{\dagger}$, A McKibben*, A Adams*, M Goldner*, JJ Marini* and L Gattinoni \\ 1st. Anestesia e Rianimazione, Osp. Maggiore Policlinico-IRCCS, via F. Sforza, 35, 20122, Milano, Italy; ${ }^{*}$ University of \\ Minnesota, St. Paul, MN, USA; 'Dip. di Scienze Cliniche e Biologiche, Univ. dell'Insubria, Varese, Italy
}

Introduction: During Acute Respiratory Distress Syndrome (ARDS), the lung density increases along a gravity gradient, causing a hydrostatic pressure gradient, even if the edema is homogeneously distributed. In an oleic acid induced ARDS experimental model, we studied by CT scan the influence of lung hydrostatic pressure on regional pleural pressure.

Methods: ARDS was induced in eight sedated, paralyzed and mechanically-ventilated dogs, by an oleic acid dose $(0.075 \mathrm{mg} / \mathrm{kg})$ injected in the pulmonary circulation. Before and after ARDS induction, a CT scan basal section was taken, and pleural pressure was directly measured throughout two flat pressure sensors (wafers) positioned into the pleural space, in the most upper part and in the most dependent part of the lung. We calculated the superimposed pressure (SP) by mean density $(\rho)$ and height (h) of the CT scan section, according to the following formula: $S P=\rho \times h$ [1]. SP represents an estimation of hydrostatic pressure that weighs at the lowest level of the CT section.
Results: After oleic acid, SP is significantly increased (from 5.0 \pm 0.3 to $10.5 \pm 1.9 \mathrm{cmH}_{2} \mathrm{O}, P<0.01$ ), and pleural pressure gradient (calculated as the difference between the pressure measured by the lower and the upper wafer) has a similar trend (from $6.5 \pm 2.0$ to $10.0 \pm 4.2 \mathrm{cmH}_{2} \mathrm{O}$, $P<0.01)$. The SP sterno-vertebral gradient $(\Delta S P)$ is significantly correlated with the pleural pressure gradient $(\Delta \mathrm{Ppl})$ ( $P=0.01 ; r=0.76)$, according to the following equation: $\Delta \mathrm{Ppl}\left[\mathrm{cmH}_{2} \mathrm{O}\right]=(1.33 \pm 1.21)+(0.74 \pm 0.23)^{*} \Delta \mathrm{SP}$ $\left[\mathrm{cmH}_{2} \mathrm{O}\right]$.

Conclusion: Pleural pressure seems to change as a function of superimposed pressure, and both these pressures change as a function of the sterno-vertebral level. However, the slope between $\Delta \mathrm{Ppl}$ and $\Delta \mathrm{SP}$ is lower than 1 because also other variables (thorax shape, regional compliance, etc.) probably influence the SP effect on the pleural pressure changes.

\author{
Reference: \\ 1. Pelosi P et al:: Am J Respir Crit Care Med 1994, 149:8-13.
}




\title{
P116 Effects of a lung recruitment maneuver keeping PEEP before and after L-Pflex on gas exchange in child ARDS patients
}

AMAS Gaudêncio, EJ Troster, LF Faria, JBS Borges, FAC Vaz, CRR Carvalho, MBP Amato and CSV Barbas

Respiratory and Pediatric ICU, Hospital das Clínicas, University of São Paulo, S.P. Brazil, Av. Dr. Eneas de Carvalho Aguiar 155- 8 andar-bloco 3, CEP-05403-900, São Paulo, SP, Brazil

PEEP titrated by the L-Pflex of static PxV curve of respiratory system associated with recruitment maneuvers improved oxygenation and mortality in ARDS patients. In order to study the effects of PEEP $2 \mathrm{cmH}_{2} \mathrm{O}$ below and above the L-Pflex without and with a recruitment maneuver on gas exchange and hemodynamic parameters, we analyzed foyr children (7 to 14 months) with ARDS criteria: acute bilateral $\mathrm{RX}$ infiltrates, $\mathrm{PaO}_{2} / \mathrm{FIO}_{2}<200$, echocardiography without left cardiac dysfunction, $(<5$ days of installation). All the children were sedated and paralyzed. After a ventilatory control period ( $\left.T V=10 \mathrm{ml} / \mathrm{kg}, \mathrm{PEEP}=5 \mathrm{cmH}_{2} \mathrm{O}\right)$, a constant flow (0.2 l/min) PxV curve was done for each child and the L-Pflex was calculated. Then the children were ventilated in VCV (6 ml/kg, RR=20/min- Servo-Siemens-300) for 30 min with PEEP $2 \mathrm{cmH}_{2} \mathrm{O}$ below the L-Pflex without recruitment maneuver, then $30 \mathrm{~min}$ with the same PEEP level associated with a recruitment maneuver ( $P C V=15$ $\mathrm{cmH}_{2} \mathrm{O}$, PEEP between 30 and $40 \mathrm{cmH}_{2} \mathrm{O}$, according to an immediately previous thoracic CT-scan for each child that showed less than 5\% of the lung area between -100 and $+100 \mathrm{HU})$. Then the children were ventilated for 30 more min with PEEP $2 \mathrm{cmH}_{2} \mathrm{O}$ above the L-Pflex without a recruitment maneuver, and finally $30 \mathrm{~min}$ with the same PEEP level associated with the recruitment maneuver.

Results: See Table.

Conclusion: A recruitment maneuver according to the findings of the thoracic CT-scan improved oxygenation mainly after PEEP set $2 \mathrm{cmH}_{2} \mathrm{O}$ above the L-Pflex of the $\mathrm{PxV}$ curve, improved $\mathrm{CO}_{2}$ exchange without hemodynamic impairment, in child ARDS patients.

\begin{tabular}{|c|c|c|c|c|c|}
\hline Patients $n=4$ & $\begin{array}{c}30 \mathrm{~min} \\
\text { PEEP } 2 \mathrm{cmH}_{2} \mathrm{O} \\
<\text {-Pflex without R.M. }\end{array}$ & $\begin{array}{c}30 \mathrm{~min} \\
\text { PEEP } 2 \mathrm{cmH}_{2} \mathrm{O} \\
<\text { L-Pflex with R.M. }\end{array}$ & $\begin{array}{c}30 \mathrm{~min} \\
\text { PEEP } 2 \mathrm{cmH}_{2} \mathrm{O} \\
>\text { L-Pflex without R.M. }\end{array}$ & $\begin{array}{c}30 \mathrm{~min} \\
\text { PEEP } 2 \mathrm{cmH}_{2} \mathrm{O} \\
>\text { L-Pflex with R.M. }\end{array}$ & \\
\hline $\operatorname{PEEP}\left(\mathrm{cmH}_{2} \mathrm{O}\right)$ & $16.5 \pm 4.1$ & $16.5 \pm 4.1$ & $20.00 \pm 3.2$ & $20.00 \pm 3.2$ & \\
\hline $\mathrm{PaO}_{2} / \mathrm{FIO}_{2}$ & $180.7 \pm 64.8^{*}$ & $227.0 \pm 57.6^{\star}$ & $219.42 \pm 51.7^{\star \star}$ & $292.0 \pm 73.8^{\star \star}$ & $P=0.02$ \\
\hline $\mathrm{PaCO}_{2}(\mathrm{mmHg})$ & $101.7 \pm 29$ & $107.7 \pm 2.5$ & $122.17 \pm 32.8^{*}$ & $91.15 \pm 4.84^{*}$ & $P=0.01$ \\
\hline MAP $(\mathrm{mmHg})$ & $69 \pm 14.5$ & $62.5 \pm 5.4$ & $62.75 \pm 9.2$ & $64.25 \pm 9.32$ & n.s. \\
\hline HR & $151 \pm 13.32$ & $153 \pm 13.69$ & $152.25 \pm 11.5$ & $149.5 \pm 15.16$ & n.s. \\
\hline
\end{tabular}

\section{P117 Effects of artificial changes in chest wall compliance on respiratory mechanics and gas exchange in patients with acute lung injury (ALI)}

\author{
N Bottino, M Panigada, D Chiumello, P Pelosi* and L Gattinoni \\ 1st. Anestesia e Rianimazione, Osp. Maggiore Policlinico-IRCCS, via F. Sforza, 35, 20122, Milan, Italy; ${ }^{*}$ Dip. di Scienze \\ Cliniche e Biologiche, Univ. dell'Insubria, Varese, Italy
}

In ALI patients, the prone position induces a decrease in chest wall compliance significantly correlated with oxygenation improvement. We studied the effects on respiratory mechanics and oxygenation of artificial change of chest wall compliance (obtained positioning a $10 \mathrm{~kg}$ 'sand bag' (SB) upon the upper thorax) in 11 supine mechanically ventilated $\mathrm{ALI}$ patients (volume control mode, $P E E P=14 \pm 3 \mathrm{cmH}_{2} \mathrm{O}$, i.e., 2-5 $\mathrm{cmH}_{2} \mathrm{O}$ above the $\mathrm{PV}$ curve inflection point (' $\left.P_{\text {flex }} P E E P '\right), V_{T}=556 \pm 137 \mathrm{ml}$, $\mathrm{RR}=17 \pm 4 \mathrm{br} / \mathrm{min}, \mathrm{V}_{\mathrm{E}}=9.1 \pm 1.3 \mathrm{~L} / \mathrm{min}, \mathrm{F}_{\mathrm{i}} \mathrm{O}_{2}=80 \pm 22 \%$ ).

We measured gas exchange, hemodynamics, total respiratory system compliance $\left(\mathrm{C}_{\mathrm{RS}}\right)$ partitioned into its lung $\left(C_{L}\right)$ and chest wall $\left(C_{C W}\right)$ components (end-inspiratory occlusion and esophageal balloon technique), end-expi- ratory lung volume (EELV; helium dilution), abdominal ventilation distribution $\left(\mathrm{V}_{\text {abd }} / \mathrm{V}_{\text {tot }} ;\right.$ Respitrace ${ }^{\circledR}$, SensorMedics) and intraabdominal pressure (IAP; intrabladder technique).

SB positioning led to an EELV decrease (from 1.22 \pm 0.75 to $0.99 \pm 0.66 \mathrm{I}, P<0.05$ ) and, interestingly, to an increase of $\mathrm{C}_{\mathrm{CW}}$ and $\mathrm{C}_{\mathrm{RS}}$ (from $43 \pm 23$ to $58 \pm 35$ and from $34 \pm 15$ to $40 \pm 18 \mathrm{ml} / \mathrm{cmH}_{2} \mathrm{O}$, respectively, $\left.P<0.05\right)$. Mean $\mathrm{PaO}_{2}$, $\mathrm{C}_{\mathrm{L}}$ and $\mathrm{V}_{\mathrm{abd}} / \mathrm{V}_{\text {tot }}$ did not change, even if patients who improved oxygenation were the same who reduced their $\mathrm{C}_{\mathrm{CW}} \quad(\mathrm{r}=0.78, P<0.01)$ and increased $\mathrm{C}_{\mathrm{L}} \quad(\mathrm{r}=0.64$, $P<0.05)$ and $V_{\text {abd }} / \mathrm{V}_{\text {tot }}(r=0.68, P<0.05)$. Moreover, patients with lower baseline IAP had a greater $C_{\mathrm{CW}}$ reduction $(r=0.60, P<0.05)$. 
We tested SB positioning also at an higher PEEP level (5 $\mathrm{cmH}_{2} \mathrm{O}$ above ' $\mathrm{P}_{\text {flex }}$ PEEP'): increasing PEEP induced an increase of EELV (from $1.22 \pm 0.75$ to $1.37 \pm 0.82$, $P<0.05), \mathrm{V}_{\text {abd }} / \mathrm{V}_{\text {tot }}$ (from $41 \pm 19$ to $\left.50 \pm 20 \%, P<0.05\right)$ and $\mathrm{PaO}_{2}$ (from $100 \pm 24$ to $121 \pm 38 \mathrm{mmHg}, P<0.05$ ) but, at this higher PEEP level, after SB application, oxygenation decreased in all patients (from $121 \pm 38$ to $105 \pm 30$ $\mathrm{mmHg}, P<0.05)$.
Conclusion: Effects of artificial change of chest wall compliance depend on PEEP level and the patient's pathophysiological characteristics: in patients with low IAP values, SB positioning could decrease the regional compliance of upper thorax and favor a caudal displacement of the diaphragm, increasing the ventilation distribution to the abdominal compartment. This can result in an oxygenation improvement, probably due to a decrease of 'true' shunt and/or a more homogeneous ventilation to perfusion ratio.

\title{
P118 The lower inflection point of the inspiratory pressure-volume curve overestimates optimal PEEP in surfactant-treated immature lambs
}

\author{
J Ingimarsson*, LJ Björklund ${ }^{\dagger}$, A Larsson and Olof Werner ${ }^{*}$ \\ Departments of *Anaesthesiology and ${ }^{+}$Pediatrics, University Hospital, Lund, Sweden; and ${ }^{\star}$ Department of Anaesthesiology, \\ Gentofte University Hospita, Hellerup, Denmark
}

Introduction: Static inspiratory pressure-volume (P-V) curves are typically S-shaped with a lower (LIP) and an upper inflection point (UIP), probably because of successive recruitment of lung units. It is often said that PEEP should be set above LIP to avoid derecruitment during expiration. However, as shown in a rabbit model [1], the effect of PEEP on lung volume is determined by the expiratory part of the P-V loop, which has a markedly different shape.

Methods: Eleven lambs with gestational age 119-141 days (term $=145$ days) delivered by cesarean section were given surfactant and subsequently mechanically ventilated. Using an interrupter technique, static P-V loops were recorded between an end-expiratory pressure of a) 0 $\mathrm{cmH}_{2} \mathrm{O}$ (ZEEP) or b) $5 \mathrm{cmH}_{2} \mathrm{O}$ and an inspiratory pressure of $35 \mathrm{cmH}_{2} \mathrm{O}$.

Results: Loops obtained during insufflation from ZEEP had a large hysteresis; LIP was $16-21 \mathrm{cmH}_{2} \mathrm{O}$, while UIP was above $30 \mathrm{cmH}_{2} \mathrm{O}$, if seen at all (Fig. 1 loop A) and the expiratory limbs showed a high deflation stability down to a pressure of $6-11 \mathrm{cmH}_{2} \mathrm{O}$ with a steep segment at lower pressures. Loops from $5 \mathrm{cmH}_{2} \mathrm{O}$ had a nearly linear inspiratory limb, and much less hysteresis (Fig. 1 loop B).

Conclusion: In preterm lambs with fully-recruited lung volume, derecruitment can be avoided by a much lower
Figure 1

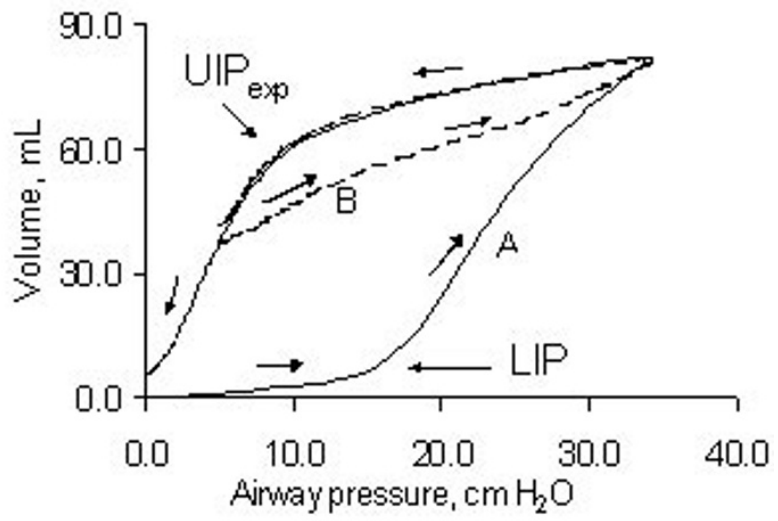

Loops at 15 min of age in a 132 day GA lamb. A: loop from ZEEP; B: loop from positive end-expiratory pressure $\left(5 \mathrm{cmH}_{2} \mathrm{O}\right)$.

PEEP than indicated by LIP. During mechanical ventilation of immature subjects, the upper inflection point of the expiratory limb $\left(\mathrm{UIP}_{\text {exp }}\right)$ is probably a better indicator of optimal PEEP than LIP of the inspiratory curve.

\section{Reference:}

1. Rimensberger et al.: Crit Care Med 1999, 27:1946-1952.

\section{P119 Effect of different tidal volumes and PEEP levels on gas exchange and FRC}

\section{Chiumello*, P Pelosi*, E Calvi*, P Taccone*, A Aliverti, R Dellaca', S Lanzi and L Gattinoni*}

Dipartimento di Bioingegneria, Politecnico di Milano, Italy; *Istituto di Anestesia e Rianimazione, Ospedale Policlinico, IRCCS, Universita' di Milano, Italy

The effect of PEEP on improving oxygenation and FRC is well known [1], not so the effects of PEEP and tidal volumes on gas exchange and FRC.

The aim of this study was to evaluate the effect of three different levels of tidal volume $(300,600,900 \mathrm{ml})$ and
PEEP $\left(5,10,15 \mathrm{cmH}_{2} \mathrm{O}\right)$ on gas exchange and FRC. We studied nine intubated sedated and paralyzed patients (5 males) in controlled volume ventilation with respiratory failure in the early phase of the disease. Baseline clinical characteristics: age $60 \pm 19$ years, weight $72 \pm 10 \mathrm{~kg}$, $\mathrm{PaO}_{2} / \mathrm{FiO}_{2} 240 \pm 101$, PEEP level of $10 \mathrm{cmH}_{2} \mathrm{O}$. FRC was 


\begin{tabular}{llccc} 
& TV & $300 \mathrm{ml}$ & $600 \mathrm{ml}$ & $900 \mathrm{ml}$ \\
\hline PEEP & & & & \\
$5 \mathrm{cmH}_{2} \mathrm{O}$ & $\mathrm{PaO}_{2}$ & $67 \pm 12$ & $96 \pm 20$ & $102 \pm 41 \mathrm{mmHg}$ \\
& $\mathrm{FRC}$ & $740 \pm 503$ & $794 \pm 355$ & $870 \pm 392 \mathrm{ml}$ \\
$10 \mathrm{cmH}_{2} \mathrm{O}$ & $\mathrm{PaO}_{2}$ & $87 \pm 33$ & $116 \pm 44$ & $130 \pm 63 \mathrm{mmHg}$ \\
& $\mathrm{FRC}$ & $1032 \pm 568$ & $1057 \pm 427$ & $1189 \pm 613 \mathrm{ml}$ \\
$15 \mathrm{cmH}_{2} \mathrm{O}$ & $\mathrm{PaO}_{2}$ & $106 \pm 58$ & $144 \pm 84$ & $147 \pm 82 \mathrm{mmHg}$ \\
& $\mathrm{FRC}$ & $1249 \pm 626$ & $1310 \pm 588$ & $1435 \pm 670 \mathrm{ml}$ \\
\hline
\end{tabular}

ANOVA two way RM: $P<0.05$ either PEEP or TV, no significant interaction between PEEP and TV. measured with the Helium dilution technique during an end-expiratory pause. Data are expressed as mean \pm sd.

Moreover, we found a significant correlation between $\mathrm{PaO}_{2}$ and $\mathrm{FRC}$ in every considered conditions $(r=0.61$, $P<0.05)$.

Our results showed that both the level of PEEP and the level of tidal volume, but not their interaction, have a significant effect on improving FRC and gas exchange.

\section{Reference:}

1. Ranieri et al:: Am Rev Respir Dis 1991, 144:544-551.

\title{
P120 Beneficial effects of lung recruitment with PEEP after cardiac surgery
}

\author{
PT Morley, K Rees, JJ Presneill, M Rowlands, JF Cade and J Tatoulis \\ Cardiothoracic Intensive Care, The Royal Melbourne Hospital, Victoria, 3050, Australia
}

Introduction: Delayed extubation and pulmonary atelectasis increase resource utilization after cardiac surgery. Lung recruitment techniques may help prevent these problems.

Objectives: To study the cardiovascular and respiratory effects of a recruitment maneuver using PEEP in postoperative cardiac surgical patients.

Methods: A prospective, randomized, double-blind study was performed. All patients were stabilized on a standard ventilatory protocol $\left(10 \mathrm{ml} / \mathrm{kg}\right.$ at $10 / \mathrm{min}$; with $\mathrm{FIO}_{2}$ of 0.8 and $P E E P=5 \mathrm{cmH}_{2} \mathrm{O}$ ). PEEP was either left at $5 \mathrm{cmH}_{2} \mathrm{O}$ (control) or increased (1 $\mathrm{cmH}_{2} \mathrm{O} / \mathrm{min}$ ) until $15 \mathrm{cmH}_{2} \mathrm{O}$ (recruitment), when cardiovascular endpoints were reassessed. PEEP was then returned to baseline at the same rate. Primary endpoints were the proportion of patients achieving predetermined oxygenation criteria for extubation $\left(\mathrm{PaO}_{2} \geq 70 \mathrm{mmHg}\right.$ on $\left.\leq 50 \% \mathrm{FiO}_{2}\right)$ at $1 \mathrm{~h}$, and time to extubation.
Results: 100 patients (mean age 63 years; 79\% male) were randomized to either recruitment $(n=55)$ or control $(n=45)$. In the recruitment group, at peak PEEP there was a small but statistically significant increase in PAWP and CVP, with a small decrease in mean BP and cardiac index.

Time to extubation was similar in each group. Oxygenation criteria for extubation at $1 \mathrm{~h}$ were achieved by $93 \%$ of the total recruitment group versus $82 \%$ of controls $(P=0.13)$. In the subgroup with an initial $\mathrm{PaO}_{2} / \mathrm{FIO}_{2}$ ratio $<300,94 \%$ achieved oxygenation criteria for extubation at $1 \mathrm{~h}$ versus $61 \%$ of controls $(P<0.01)$. Chest radiographs taken after completion of the protocol showed significantly less basal pulmonary collapse in the recruitment group (20\% versus $53 \%$ in controls; $P<0.01$ ).

Conclusion: The use of this incremental PEEP maneuver after cardiac surgery improves pulmonary gas exchange, probably via recruitment of otherwise collapsed pulmonary segments. These benefits are more pronounced in those patients with initially poor gas exchange.

\section{P121 Effects of PEEP and tidal volume on elastances and distribution of volume changes of the different chest wall compartments}

\section{A Aliverti, R Dellacà, A Lo Mauro, E Carlesso, W Del Frate, P Pelosi, D Chiumello* and A Pedotti}

Dipartimento di Bioingegneria, Politecnico di Milano, Italy; */stituto di Anestesia e Rianimazione, Università di Milano, Ospedale Maggiore IRCCS, Milano, Italy

We used optoelectronic plethysmography [1] to analyze six sedated and paralyzed ALI/ARDS patients receiving continuous positive pressure ventilation (CPPV) with different settings of PEEP $\left(5,10,15 \mathrm{cmH}_{2} \mathrm{O}\right)$ and tidal volume (TV, $300,600,900 \mathrm{ml})$. The aim of the present work is to study the effects of PEEP and TV on volume distribution in three different chest wall compartments (upper thorax, $\mathrm{V}_{\mathrm{UT}}$, lower thorax, $\mathrm{V}_{\mathrm{LT}}$ and abdomen, $\mathrm{V}_{\mathrm{AB}}$ ) and on their elastances $\left(E_{U T}, E_{L T}\right.$ and $E_{A B}$, respectively) assessed by the end-inspiratory occlusion technique. Results are expressed in the table as mean $\pm S D$.

We found that both PEEP and TV, but not their interaction, have an effect on volume distribution in the UT and AB $(P<0.05)$. At increasing PEEP and TV, the increased abdominal and decreased upper thoracic contribution to inspired volume were associated to a decreased compliance of the UT. Finally, we found significant $(P<0.001)$ 
Critical Care Vol 4 Suppl 1 20th International Symposium on Intensive Care and Emergency Medicine

\begin{tabular}{lccccccc} 
PEEP $\left(\mathrm{cmH}_{2} \mathrm{O}\right)$ & TV $(\mathrm{ml})$ & Vut $(\% \mathrm{TV})$ & Vlt $(\% \mathrm{TV})$ & Vab $(\% \mathrm{TV})$ & Eut $_{\left(\mathrm{cmH}_{2} \mathrm{O} / \mathrm{L}\right)}$ & $\left.\mathrm{Elt}^{(\mathrm{cmH}} \mathrm{cm}_{2} \mathrm{~L}\right)$ & $\left.\mathrm{Eab}^{(\mathrm{cmH}} \mathrm{H}_{2} \mathrm{O} / \mathrm{L}\right)$ \\
\hline 5 & 300 & $39.4 \pm 14.4$ & $10.1 \pm 5.3$ & $50.5 \pm 14.0$ & $76.9 \pm 26.3$ & $378.1 \pm 266.9$ & $63.6 \pm 37.6$ \\
5 & 600 & $32.5 \pm 9.5$ & $11.1 \pm 3.3$ & $56.4 \pm 11.4$ & $76.8 \pm 25.3$ & $211.4 \pm 63.6$ & $45.3 \pm 23.4$ \\
5 & 900 & $27.7 \pm 7.6$ & $11.1 \pm 4.0$ & $61.2 \pm 9.1$ & $92.3 \pm 47.8$ & $180.3 \pm 73.7$ & $36.3 \pm 21.2$ \\
10 & 300 & $31.7 \pm 8.8$ & $10.6 \pm 2.3$ & $57.7 \pm 9.9$ & $69.0 \pm 18.8$ & $220.5 \pm 87.6$ & $46.9 \pm 39.9$ \\
10 & 600 & $26.7 \pm 6.8$ & $10.1 \pm 3.0$ & $63.1 \pm 8.5$ & $85.2 \pm 19.6$ & $230.1 \pm 70.0$ & $39.4 \pm 14.5$ \\
10 & 900 & $24.9 \pm 6.2$ & $11.1 \pm 3.2$ & $64.0 \pm 8.2$ & $96.7 \pm 7.4$ & $190.9 \pm 51.0$ & $31.9 \pm 11.2$ \\
15 & 300 & $26.2 \pm 5.4$ & $10.4 \pm 4.5$ & $63.4 \pm 7.9$ & $92.2 \pm 10.2$ & $344.3 \pm 231.0$ & $34.1 \pm 12.1$ \\
15 & 600 & $24.8 \pm 6.7$ & $11.5 \pm 3.0$ & $63.7 \pm 8.2$ & $112.9 \pm 20.9$ & $254.7 \pm 84.9$ & $42.6 \pm 11.1$ \\
15 & 900 & $23.7 \pm 6.4$ & $10.8 \pm 3.6$ & $65.5 \pm 9.0$ & $126.7 \pm 33.1$ & $251.5 \pm 50.5$ & $43.5 \pm 17.6$ \\
\hline
\end{tabular}

exponential relationships between UT and $A B$ contributions to $\mathrm{TV}$ and $\mathrm{E}_{\mathrm{UT}}$.

We conclude that at high PEEP and TV, the elastance of the upper thorax decreases and it causes a more 'abdominal' distribution of TV.

Reference:

1. Aliverti $A$, Dellacà $R$, Pelosi $P$, Chiumello $D$, Pedotti $A$, Gattinoni L: Opto-electronic plethysmography in intensive care patients. $\mathrm{Am} \mathrm{J}$ Resp Crit Care Med (in press).

\section{P122 Effects of PEEP above the L-Pflex on gas exchange, hemodynamic and gastric tonometry in ARDS patients}

\section{CSV Barbas, E Silva, C Hoelz, AG Garrido, EC Meyer and E Knobel}

ICU, Hospital Israelita Albert Einstein, São Paulo,SP-Brazil, Av. Albert Einstein 627/701-5 andar-CTI-adultos-CEP-105651-901, São Paulo, SP-Brazil

PEEP titrated by the L-Pflex of static PxV curve of respiratory system improved oxygenation and mortality in ARDS patients. In order to study the acute effects of PEEP on oxygenation, $\mathrm{CO}_{2}$ exchange, hemodynamic parameters and gastric tonometry we analyzed six ARDS patients $(<5$ days of installation) after 30 min on PEEP of $5 \mathrm{cmH}_{2} \mathrm{O}$, then 30 min on PEEP $2 \mathrm{cmH}_{2} \mathrm{O}$ above the L-Pflex and then $30 \mathrm{~min}$ after PEEP of $5 \mathrm{cmH}_{2} \mathrm{O}$ again. They were all sedated and paralyzed. A Swan-Ganz catheter with a continuos cardiac output $\left(\right.$ Baxter $\left.^{\circledR}\right)$ and a continuos gastric tonometer $\left(\right.$ Tonocap $^{\circledR}$ ) were inserted in each patient. The tidal volume and respiratory rate were kept constant (8 $\mathrm{ml} / \mathrm{kg}$ and 20/min- VCV-Ventilation-Servo-Siemens-300). L-Pflex was titrated by the static PxV curve of the respiratory system (random volumes).

Results: See Table.

Conclusion: PEEP titrated by the L-Pflex of the static PxV curve of the respiratory system improved oxygenation without impairing global as well as regional hemodynamic parameters in ARDS patients.

\begin{tabular}{|c|c|c|c|c|}
\hline $\begin{array}{l}\text { Patients } \\
\mathrm{N}=6\end{array}$ & $\begin{array}{c}30 \text { min after } \\
\text { PEEP=5 } \mathrm{cmH}_{2} \mathrm{O}\end{array}$ & $\begin{array}{c}30 \mathrm{~min} \text { after } \\
\text { PEEP } 2 \mathrm{cmH}_{2} \mathrm{O}>\text { L-Pflex }\end{array}$ & $\begin{array}{c}30 \text { min after } \\
\text { PEEP }=5 \mathrm{cmH}_{2} \mathrm{O}\end{array}$ & \\
\hline $\operatorname{PEEP}\left(\mathrm{cmH}_{2} \mathrm{O}\right)$ & $5 \pm 0$ & $12.6 \pm 3.3$ & $5 \pm 0$ & $P<0.05$ \\
\hline Minute ventilation (L/min) & $10.37 \pm 2.74$ & $10.37 \pm 2.74$ & $10.37 \pm 2.74$ & n.s. \\
\hline $\mathrm{PaO}_{2} / \mathrm{FIO}_{2}$ & $61.4 \pm 18.9$ & $131.6 \pm 40.1$ & $90.2 \pm 39.4$ & $P<0.001$ \\
\hline $\mathrm{PaCO}_{2}(\mathrm{mmHg})$ & $43.6 \pm 15.4$ & $41.4 \pm 17.1$ & $38.6 \pm 18.9$ & n.s \\
\hline $\mathrm{PgCO}_{2}(\mathrm{mmHg})$ & $50.4 \pm 9.8$ & $49.8 \pm 16.8$ & $49.4 \pm 17.4$ & n.s \\
\hline Arterial Lactate $(\mathrm{mg} / \mathrm{dL})$ & $22.2 \pm 14.6$ & $21.9 \pm 5.7$ & $22.6 \pm 5.9$ & n.s \\
\hline Cardiac Index & $4.8 \pm 1.2$ & $4.0 \pm 1.2$ & $4.6 \pm 1.4$ & $P=0.06$ \\
\hline Mean arterial pressure $(\mathrm{mmHg})$ & $71.7 \pm 12.7$ & $80.3 \pm 14.9$ & $72.0 \pm 11.7$ & n.s. \\
\hline
\end{tabular}




\section{P123 Influence of different PEEP levels and tidal volumes on the regional nonaerated tissue: experimental study}

N Bottino, G Eccher, P Pelosi ${ }^{\dagger}$, A McKibben*, A Adams*, M Goldner*, JJ Marini* and L Gattinoni

1st. Anestesia e Rianimazione, Osp. Maggiore Policlinico-IRCCS, via F. Sforza, 35, 20122, Milano, Italy; *University of

Minnesota, St Paul, MN, USA; ${ }^{\dagger}$ Dip. Scienze Cliniche e Biologiche, Univ. dell'Insubria, Varese, Italy

Introduction: In the Acute Respiratory Distress Syndrome (ARDS), although the edema is homogeneously distributed, a hydrostatic pressure gradient squeezes the gas out from the alveoli, causing a lung density increase along a gravity gradient. We studied by CT scan, in an oleic acid induced ARDS experimental model, the influence of different $\mathrm{V}_{\mathrm{T}}$ and PEEP levels on the regional nonaerated tissue during volume-controlled ventilation.

Methods: ARDS was induced in six sedated, paralyzed and mechanically-ventilated dogs by an oleic acid dose $(0.075 \mathrm{mg} / \mathrm{kg})$ injected into the pulmonary circulation. After ARDS induction, the volume-controlled ventilation was maintained, changing three tidal volumes $\left(\mathrm{V}_{\mathrm{T}}\right)(12,24$ and $36 \mathrm{ml} / \mathrm{kg}$ ) and two PEEP levels ( 5 and $15 \mathrm{cmH}_{2} \mathrm{O}$ ). During each of the six randomized steps a CT scan section has been taken, at end-expiration and end-inspiration, $5 \mathrm{~cm}$ above the diaphragm. For data analysis, we divided the CT scan section into an upper and a lower part. The nonaerated tissue mass of the upper or lower region was calculated from the densities $(\rho)(-200 \mathrm{H}<\mathrm{CT}$ $<+100 \mathrm{H})$ and the volume $(\mathrm{V})$, according to the formula $m=\rho \times V$ [1]; then it was standardized for the area of each lung region. To evaluate the effect of $\mathrm{V}_{\mathrm{T}}$, PEEP level and lung region (upper/lower) on the amount of nonaerated tissue, we performed analysis of variance, in condition of end-expiration and end-inspiration, respectively.

Results: The behavior of nonaerated tissue $\left(\mathrm{g} / \mathrm{cm}^{2}\right)$ is shown in the table (data expressed as mean $\pm S D$ ).

The amount of nonaerated tissue is greater in the lower region than in the upper one, in both end-expiration and end-inspiration $(P<0.01)$. In both regions PEEP and $V_{T}$ independently affect the amount of nonaerated tissue $(P<0.01)$. There is interaction between PEEP and $V_{T}$ only at end-inspiration (end-expiration: $P=$ n.s.; end-inspiration: $P<0.01$ )

Conclusion: The effects of PEEP level and $V_{T}$ on lung recruitment are greater in dependent lung regions and at end-inspiration.

\section{Reference:}

1. Pelosi P et al:: Am J Respir Crit Care Med 1994, 149:8-13.

\begin{tabular}{llllllll} 
& & 5 , low $\mathrm{V}_{\mathrm{T}}$ & 5 , normal $\mathrm{V}_{\mathrm{T}}$ & 5 , high $\mathrm{V}_{\mathrm{T}}$ & 15 , low $\mathrm{V}_{\mathrm{T}}$ & 15 , normal $\mathrm{V}_{\mathrm{T}}$ & 15, high $\mathrm{V}_{\mathrm{T}}$ \\
\hline upper & end-exp. & $0.14 \pm 0.08$ & $0.12 \pm 0.07$ & $0.17 \pm 0.14$ & $0.06 \pm 0.02$ & $0.05 \pm 0.03$ & $0.06 \pm 0.02$ \\
& end-insp. & $0.06 \pm 0.03$ & $0.05 \pm 0.03$ & $0.05 \pm 0.03$ & $0.05 \pm 0.02$ & $0.05 \pm 0.03$ & $0.04 \pm 0.02$ \\
\multirow{3}{*}{ lower } & end-exp. & $0.81 \pm 0.05$ & $0.70 \pm 0.11$ & $0.55 \pm 0.19$ & $0.29 \pm 0.14$ & $0.20 \pm 0.10$ & $0.12 \pm 0.04$ \\
& end-insp. & $0.68 \pm 0.05$ & $0.42 \pm 0.07$ & $0.21 \pm 0.17$ & $0.24 \pm 0.12$ & $0.10 \pm 0.05$ & $0.05 \pm 0.03$ \\
\hline
\end{tabular}

P124 Computer tomography of chest organs in the diagnosis of acute respiratory distress-syndrome of patients with severe brain injury

\section{NV Agafonova, SA Kravtsov and EP Rodionov}

State Scientific and Clinical Center of Coal Miners' Health Protection, Leninsk-Kuznetsky, Russia, 652509

Purpose: Early diagnosis of acute injuries of lungs in patients with severe brain injury (SBI) during acute period providing, with the comparison of results in dynamic control, reduction of radial load and of examination time.

Materials and methods: We examined 25 patients with $\mathrm{SBI}$ (in admission the severity rate was less than 8 points according to the Glasgow scale) at 1, 2, 3, 5, 7 and 14 days. We developed the algorhythm of patients examination: high resolution computer tomography (HRCT) of four levels with assessment of densitometric index in four points of each level.
Results: Roentgenologic signs of $96 \%$ patients during 1 day; from these patients $62.5 \%$ had first rate with the increase of density index by $13.7 \%, 37.5 \%$ patients had second rate with the increase of density index by $25.2 \%$ in comparison with density index. Main localization of pathologic changes registered in posterior basal parts of the lungs. Pleural exudate was diagnosed in $50 \%$, hydrothorax in $4 \%$, aspiration pneumonia in $8 \%$ patients.

Conclusion: The proposed method, in contrast to standard CT, allows the appreciation of the revealed changes, to diagnose the early symptoms of ARDS on the first day, to analyze the development of pathologic in dynamics and to conduct the treatment correction in time. 


\section{P125 Partial liquid ventilation combined with two different gas ventilation strategies in acute lung} injury in piglets

G Zobel, S Rödl, B Urlesberger, I Knez and D Dacar

Departments of Pediatrics, Neonatology, and Cardiac Surgery, University of Graz, Austria

Objectives: To investigate the effects of two different gas ventilation strategies during partial liquid ventilation (PLV) on gas exchange and lung mechanics in acute lung injury.

Design: Prospective, randomized, experimental study.

Setting: University research laboratory.

Subjects: Fourteen piglets weighing $8.9 \pm 0.2 \mathrm{~kg}$.

Interventions: Acute lung injury was induced by the infusion of oleic acid and repeated lung lavages with $0.9 \%$ $\mathrm{NaCl}(20 \mathrm{ml} / \mathrm{kg})$. After a stabilization period of $30 \mathrm{~min}$ the animals were randomized to two groups with different PEEP levels and tidal volumes (Group A: Vt $>12.5 \mathrm{ml} / \mathrm{kg}$, PEEP $6 \mathrm{cmH}_{2} \mathrm{O}$; Group B: Vt $9 \mathrm{ml} / \mathrm{kg}$, PEEP $12 \mathrm{cmH}_{2} \mathrm{O}$ ). The pre-oxygenated and warmed perfluorocarbon liquid $(30 \mathrm{ml} / \mathrm{kg}$ ) was instilled into the trachea over $15 \mathrm{~min}$ without changing the ventilator settings.

Measurements and main results: Airway pressures, tidal volumes, static respiratory compliance, inspiratory and expiratory airway resistances and arterial blood gases were measured. Data were obtained at baseline, after lung injury and at 60 and $120 \mathrm{~min}$ of PLV. See Table 1.

Data analysis: Values are given as mean \pm SEM. Comparisons were made by ANOVA for repeated measures. A $P$ value of $<0.05$ was considered significant. The infusion of oleic acid $(0.07 \mathrm{ml} / \mathrm{kg})$ combined with five lung lavages induced a significant reduction of $\mathrm{PaO}_{2} / \mathrm{FiO}_{2}$ from $486 \pm 24$ torr to $60 \pm 3.8$ torr $(P<0.01)$.

Conclusions: During PLV both gas ventilation strategies significantly improved oxygenation in an animal model of acute lung injury. Static compliance of the respiratory system was not different between both gas ventilation strategies. Inspiratory airway resistance was significantly higher during the low PEEP-high Vt gas ventilation strategy, whereas the high PEEP-low VT strategy resulted in significantly higher $\mathrm{PaCO}_{2}$ values.

Table 1

Blood gases and respiratory variables during PLV with two different strategies of gas ventilation.

\begin{tabular}{|c|c|c|c|c|c|}
\hline Parameters & Group & $\mathrm{BL}$ & ALI & $\mathrm{T}-60 \mathrm{~min}$ & $\mathrm{~T}-120 \mathrm{~min}$ \\
\hline $\mathrm{PaO}_{2} / \mathrm{FiO}_{2}$ (torr) & $\begin{array}{l}\text { A } \\
B\end{array}$ & $\begin{array}{l}490 \pm 30^{\star \star} \\
493 \pm 40^{\star \star}\end{array}$ & $\begin{array}{l}62 \pm 6 \\
61 \pm 125\end{array}$ & $\begin{array}{l}228 \pm 50^{*} \\
230 \pm 56^{*}\end{array}$ & $\begin{array}{l}207 \pm 34^{*} \\
245 \pm 62^{*}\end{array}$ \\
\hline Qs/Qt(\%) & $\begin{array}{l}\text { A } \\
B\end{array}$ & $\begin{array}{r}14.9 \pm 3^{\star *} \\
14 \pm 2^{\star *}\end{array}$ & $\begin{array}{l}55 \pm 4 \\
48 \pm 3\end{array}$ & $\begin{array}{l}34 \pm 6^{*} \\
32 \pm 4^{*}\end{array}$ & $\begin{array}{l}35 \pm 4^{*} \\
31 \pm 4^{*}\end{array}$ \\
\hline $\operatorname{Cstat}\left(\mathrm{ml} / \mathrm{cmH}_{2} \mathrm{O} / \mathrm{kg}\right)$ & $\begin{array}{l}\text { A } \\
B\end{array}$ & $\begin{array}{l}1.02 \pm 0.1^{\star *} \\
1.05 \pm 0.05^{\star *}\end{array}$ & $\begin{array}{l}0.52 \pm 0.05 \\
0.43 \pm 0.05\end{array}$ & $\begin{array}{l}0.63 \pm 0.08 \\
0.52 \pm 0.06\end{array}$ & $\begin{array}{l}0.59 \pm 0.08 \\
0.55 \pm 0.07\end{array}$ \\
\hline $\operatorname{Rawinsp}\left(\mathrm{cmH}_{2} \mathrm{O} / \mathrm{L} / \mathrm{s}\right)$ & $\begin{array}{l}\text { A } \\
B\end{array}$ & $\begin{array}{l}34 \pm 1.7^{*} \\
38 \pm 4^{*}\end{array}$ & $\begin{array}{l}43 \pm 5.9^{*} \\
53 \pm 7.2^{*}\end{array}$ & $\begin{array}{l}65 \pm 3.4^{* *} \\
44 \pm 5 \#\end{array}$ & $\begin{array}{l}67 \pm 5.8^{\star *} \\
43 \pm 6.1 \#\end{array}$ \\
\hline $\mathrm{Vd} / \mathrm{Vt}(\%)$ & $\begin{array}{l}A \\
B\end{array}$ & $\begin{array}{c}13.7 \pm 3.0^{\star \star} \\
11 \pm 3^{\star \star}\end{array}$ & $\begin{array}{l}37 \pm 5 \\
37 \pm 1\end{array}$ & $\begin{array}{l}35 \pm 3 \\
30 \pm 1.8\end{array}$ & $\begin{array}{l}42 \pm 2.7 \\
32 \pm 2.5 \#\end{array}$ \\
\hline $\mathrm{PaCO}_{2}$ (torr) & $\begin{array}{l}A \\
B\end{array}$ & $\begin{array}{l}41.7 \pm 1.5^{\star \star} \\
39.9 \pm 2.9^{\star \star}\end{array}$ & $\begin{array}{l}54.4 \pm 3 \\
48.3 \pm 2.2\end{array}$ & $\begin{array}{l}41.7 \pm 2.3^{\star \star} \\
64 \pm 2.4^{\star \star} \# \#\end{array}$ & $\begin{array}{l}43.8 \pm 1.1^{\star \star} \\
66 \pm 2.1^{\star \star} \# \#\end{array}$ \\
\hline
\end{tabular}

Group A: Vt $>12.5 \mathrm{ml} / \mathrm{kg}$, PEEP $6 \mathrm{cmH}_{2} \mathrm{O}$, Group B: Vt $9 \mathrm{ml} / \mathrm{kg}$, PEEP $12 \mathrm{cmH}_{2} \mathrm{O}$; ${ }^{\star} P<0.05$ vs ALI, ${ }^{\star \star} P<0.01$ vs ALI, BL: baseline; ALI: acute lung injury; $\# P<0.05$ vs group $A, \# \# P<0.01$ vs group $A$.

\section{P126 Oxygenation response to prone positioning in pulmonary and extrapulmonary ARDS}

O Akinci, M Cetin, P Ergin, F Esen, L Telci and N Cakar

Department of Anesthesiology and Intensive Care, University of Istanbul, Medical Faculty, Istanbul, Turkey

Introduction: Various etiologic factors change clinical response, morbidity, and mortality rates in ARDS. In this study, we investigated the oxygenation response of pulmonary and extrapulmonary ARDS patients to the prone position.
Methods: Thirty-two ARDS patients (Group1: $n=16$ with pulmonary causes and Group 2 : $n=16$ with extrapulmonary causes) were evaluated retrospectively. ARDS was defined according to the criteria established by the Ameri- 
Table 1

\begin{tabular}{lcc}
\hline & Group 1 $\left(\mathrm{PaO}_{2} / \mathrm{FiO}_{2}\right)$ & Group 2 $\left(\mathrm{PaO}_{2} / \mathrm{FiO}_{2}\right)$ \\
\hline Supine $P$ & $126.8 \pm 47.8$ & $121 \pm 46.2$ \\
Prone $P$ 1st hour & $171.9 \pm 72$ & $196.6 \pm 88.2$ \\
Prone $P$ 4th hour & $225.5 \pm 84.8$ & $217.8 \pm 68.6$ \\
Prone $P$ 24th hour & $223.6 \pm 53.8$ & $216.7 \pm 92.8$ \\
\hline
\end{tabular}

can-European Consensus Conference on ARDS [1]. $\mathrm{PaO}_{2} / \mathrm{FiO}_{2}$ ratios, $\mathrm{PaCO}_{2}, \mathrm{PCWP}$ values in the supine position (baseline) and then the same values in the prone position at the 1st, 4th and 24th $\mathrm{h}$ were obtained from the medical records. ARDS, APACHE II, and MOF scores on admission, and the first day of prone positioning were calculated and compared. Statistical evaluations were performed by student t-test.

Results: Murray, APACHE II and MOF scores were similar in both groups during admission and there was no difference in oxygenation response to prone positioning between two groups (Table 1). However, the MOF score was higher in extrapulmonary ARDS on the day of prone positioning. Mortality rate was higher in the extrapulmonary group and on discharge this group had higher Murray, APACHE II and MOF scores (Table 2).
Table 2

\begin{tabular}{llccc}
\hline & & Group 1 & Group 2 & $P$ \\
\hline \multirow{4}{*}{ Murray } & on admission & $2.4 \pm 0.8$ & $2.3 \pm 0.9$ & 0.67 \\
& prone & $2.6 \pm 0.6$ & $2.3 \pm 0.5$ & 0.9 \\
& on discharge & $1.6 \pm 0.8$ & $12.4 \pm 0.8$ & $<0.01$ \\
\multirow{4}{*}{ APACHE } & on admission & $8.56 \pm 5.49$ & $12.6 \pm 9.9$ & 0.16 \\
& prone & $9.9 \pm 5.7$ & $12.4 \pm 4.2$ & 0.17 \\
& on discharge & $8.1 \pm 8.2$ & $17.8 \pm 9.0$ & $<0.05$ \\
\multirow{3}{*}{ MOF } & on admission & $1 \pm 0$ & $1.3 \pm 0.7$ & 0.09 \\
& prone & $1.4 \pm 0.7$ & $2.3 \pm 1$ & 0.012 \\
& on discharge & $1.4 \pm 0.6$ & $3.4 \pm 1.76$ & $<0.01$ \\
\hline
\end{tabular}

Conclusion: Our retrospective data suggest that oxygenation response to prone positioning was similar in both groups, but mortality rates were higher in extrapulmonary ARDS patients, which correlates with MOF scores.

\section{Reference:}

1. Bernard GR, Artigas A, Brigham KL, Carlet J, Falke K, Hudson L, Lamy M, Le Gall JR, Morris A, Sprag R, and the consensus committee: American- European Consensus Conference on ARDS. Definition, mechanism, relevant outcomes and clinical trial coordination. $A m$ J Respir Crit Care Med 1994, 149:818-824.

\section{P127 Prospective study to evaluate the kind of prone position concerning nursing, clinical outcome and material and personnel resources}

TR Neubert, R Stiletto and L Gotzen

Centre of Operative Medicine, Department of General Surgery, University of Marburg

Introduction: Acute Respiratory Distress Syndrome (ARDS) is one of the most common, potentially lethal disease processes encountered in critical care with extremely high mortality of about $60 \%$. Researchers have found that a significant improvement in gas exchange often occurs when ARDS patients are turned from the supine to the prone position. Different reasons are discussed for this effect: reduction of oxygen toxicity, recruitment of alveolar space and optimisation of postural drainage. But there are a lot of difficulties in nursing these patients and these depend on the kind of prone position. The dangers of pressure damage and oedema formation increase in the prone position. Also the possibility of suction and observation is decreased. So the major goal is to find out the best kind of prone position.

The available study is designed by physicians and nurses. Besides the question of patients' benefit concerning gas exchange, handling, the acceptance of the nurses, and economic consequences are proved.
Method/material: Patients with ARDS, or those patients identified as requiring to be nursed in the prone position with a Horowitz-Quotient $\left(\mathrm{PaO}_{2} / \mathrm{FiO}_{2}\right)<250$, were turned over into the prone position. In a randomised procedure the patients were placed into a $180^{\circ}$ prone position (face down), a $135^{\circ}$ prone position (near side position) or they were treated in a Rotation bed (RotorestR). Gas analyses defined the clinical effect of the position on gas exchange. Also changes in skin integrity, skin status and the clinical outcome of proning were documented.

Additionally, the number of nurses/physicians being involved in positioning the patient and the time taken was documented.

The prone position interval is fixed at $4-6 \mathrm{~h}$ depending on clinical data and the personnel situation.

End of the positioning-treatment is defined by clinical data and a Horowitz quotient $>300$. 
Results: Twenty-two patients were positioned in the near side prone position. Eight patients were positioned in the $180^{\circ}$-position and five patients were treated in the rotation bed.

The distribution of the patient identified as requiring to be nursed in prone position shows the preferences of the prone position. In handling, nursing and observing, the near side position is the preferred prone position. The clinical outcome is comparable to the other forms of pronepositioning. In comparison with the other kinds of position the risk of complication (skin damages, oedema formation, lost of catheter or tube) is very small. On average you need one physician and two very well-introduced nurses for this positioning. So at every point of time, position changing can occur. For the face down position $\left(180^{\circ}\right)$ on average you need four to five well-introduced persons at minimum for a position changing. Additionally you need sufficient place to range two beds side by side. The possibility of observing, nursing and suctioning is less in the face down position than in the near side position. Our findings show a similar clinical effect concerning the gas exchange in the $180^{\circ}$ position as in the $135^{\circ}$ position. But for a clear position the number of researched patient is too small.
Also the patients treated in rotation bed shows a similar clinical outcome concerning the gas exchange.

But here also the handling and the economic resources necessary are incomparably high and at every time you need a special introduced nurse.

Conclusion: This study is being continued to get evident data for a clear point of view. The target is to develop evident criteria for the kind of prone position, not only in dependence of the clinical outcome but also concerning the economic and personnel possibilities in an ICU. A clinical treatment is not only orientated in doing the best for the patient but it is also limited by personnel acceptance and economic resources. The kind of treatment is established when the benefit for the patient is recognisable, when the handling is simple, does not need complicated equipment and a lot of personal resources. It must be safe and show a small quantity of complications.

These criteria, as shown by our study, are applicable for the near side position. Our goal is to develop criteria for the different kind of positioning possibilities concerning the clinical problems in gas exchange during the disease process.

\section{P128 Inhaled nitric oxide in infants and children with ARDS}

\section{G Zobel, S Rödl, M Trop and HM Grubbauer}

Department of Pediatrics, University of Graz, Austria

Objective: To evaluate the effects of inhaled nitric oxide on gas exchange and outcome in pediatric patients with acute respiratory distress syndrome (ARDS).

Design: Case series report.

Setting: Pediatric intensive care unit of a tertiary care children's hospital.

Patients: Seventeen pediatric patients with ARDS requiring mechanical ventilation with an $\mathrm{FiO}_{2}>0.5$ at a positive end-expiratory pressure $>6 \mathrm{cmH}_{2} \mathrm{O}$, and whose $\mathrm{PaO}_{2} / \mathrm{FiO}_{2}$ ratio was $<100$ torr were enrolled in this study.
Interventions: Initially inhaled (NO) was applied at 10 ppm using a microprocessor based system. A positive response after $15 \mathrm{~min}$ of NO inhalation was defined as an increase in arterial oxygen saturation $>5 \%$.

Measurements and main results: At the start of NO inhalation the oxygenation $\left(\mathrm{Ol}=\mathrm{Paw}^{*} \mathrm{FiO}_{2}{ }^{*} 100 / \mathrm{PaO}_{2}\right)$ and ventilation $\left(\mathrm{Vl}=\mathrm{PaCO}_{2}{ }^{*} \mathrm{PIP}{ }^{*} \mathrm{RR} / 1000\right)$ indices were $29 \pm 2.8$ and $43 \pm 4.1$, respectively, the $\mathrm{PaO}_{2} / \mathrm{FiO}_{2}$ ratio was $69 \pm 7$ torr, and the static compliance of the respiratory system $0.51 \pm 0.04 \mathrm{ml} / \mathrm{cmH}_{2} \mathrm{O} / \mathrm{kg}$. An initial positive response to inhaled NO was observed in $59 \%$ of patients.

Table 1

Change in oxygen saturation 15 min after starting NO inhalation, duration of MV, number of ECMO support, and outcome of pediatric patients with ARDS $(n=17)$.

\begin{tabular}{lccccc}
\hline & Number of patients & $\mathrm{SpO}_{2}(\%)$ & Duration of MV (d) & ECMO support (n) & S/NS (Mortality rate) \\
\hline Responders & 10 & $9.2 \pm 1.7^{*}$ & $22 \pm 5$ & 4 & $6 / 4(40 \%)$ \\
Nonresponders & 7 & $0.28 \pm 0.89$ & $26 \pm 11$ & 2 & $4 / 3(43 \%)$ \\
\hline
\end{tabular}

${ }^{\star} P<0.01 ; \mathrm{S}=$ survivors; $\mathrm{NS}=$ nonsurvivors; $\mathrm{MV}=$ mechanical ventilation; $\mathrm{ECMO}=$ extracorporeal membrane oxygenation. 
Conclusion: Inhaled NO significantly improves oxygenation in 59\% of infants and children with ARDS. However, an initial positive response to inhaled NO does not reduce the need for ECMO support and does not influence duration of mechanical ventilation and outcome of pediatric patients with severe ARDS.

\section{P129 Role of hyperbaric oxygen therapy (HBOT) in recovery of cardio-pulmonary function: survival of patients developing ARDS following closed chest trauma (CCT)}

\section{GG Rogatsky and EG Shifrin}

Faculty of Life Sciences, Bar-llan University, 52900 Ramat-Gan and Department of Vascular Surgery, Sourasky Medical Center, Sackler School of Medicine, Tel-Aviv University, 64239 Tel-Aviv, Israel

Many years mortality in ARDS, often complicating CCT, is still very high and reaches $70-80 \%$. In ARDS patients, hypoxia is a result of a 'vicious circle' when acute respiratory insufficiency intensifies acute cardiac insufficiency which, in turn, deepens respiratory failure.

Patients and methods: The study is based on the data obtained from 45 patients with moderate-severe CCT. During conventional treatment in ICU, after 48-72 h, in 21 patients (48\%) an ARDS developed, and three of these patients were treated with HBOT in a mono-placed barochamber. All treatments were conducted under 1.6-2.0 ata for 40-60 min each every day during 4-15 days, depending on progress of the recovery. Monitoring of cardiac function was carried out using non-invasive impedance cardiography (IC). Stroke Volume Index (SVI) and Cardiac Output Index (COI) were calculated. Respira- tory functions were checked using a gas-analyzer. All ARDS patients were divided into three groups: group $A-4$ surviving patients after conventional therapy; group B-14 deceased patients after conventional therapy, and group C-3 patients surviving after addition of HBOT.

Results: Obtained data was exposed to statistical analysis using Student's unpaired t-test and the results are presented in Table 1.

Conclusions: This study clearly shows that: 1) in ARDS patients after CCT the state of cardiac function is the factor determining development of respiratory hypoxia; 2) HBOT is a decisive treatment improving cardio-respiratory function which leads to the favorable outcome; 3) patients with CCT must be treated with HBOT just after the trauma, before ARDS has developed.

\section{Table 1}

\begin{tabular}{|c|c|c|c|c|c|c|c|c|c|c|c|c|}
\hline \multirow{2}{*}{$\frac{\text { Groups }}{A}$} & \multicolumn{3}{|c|}{$\mathrm{PaO}_{2}(\mathrm{mmHg})$-mean } & \multicolumn{3}{|c|}{ SVI $\left(\mathrm{ml} \mathrm{m}^{-2}\right)$ - mean } & \multicolumn{3}{|c|}{ COI $\left(I \mathrm{~min}^{-1} \mathrm{~m}^{-2}\right)$ - mean } & \multicolumn{3}{|c|}{$\mathrm{HR}\left(\mathrm{b} \min ^{-1}\right)$ - mean } \\
\hline & 49.7 & 57.9 & 78. $0^{\star \star \# \# ~}$ & 31.9 & 46.4 & $50.0^{\#}$ & 3.77 & 4.76 & 4.34 & 108 & $101^{\#}$ & $90^{\#}$ \\
\hline B & 61.8 & 52.3 & $40.0^{* *}$ & 33.6 & 38.2 & 27.3 & 3.14 & $4.66^{*}$ & 3.42 & 98 & $132^{*}$ & 116 \\
\hline C & 55.8 & 60.1 & $87.3^{\star \star \# \#}$ & 33.7 & $48.4^{*}$ & $49.4^{\#}$ & 3.55 & $5.25^{\star}$ & 5.09 & 103 & $105^{\#}$ & $86^{* \#}$ \\
\hline Phases & 1 & ॥ & III & I & $\|$ & III & 1 & ॥ & III & I & $\|$ & III \\
\hline
\end{tabular}

The differences between the means was considered significant if $P<0.05$ ( ${ }^{*}$ or $\left.{ }^{\#}\right), P<0.01$ ( $^{* *}$ or ${ }^{\# \#)}$. * - vs phase I; ${ }^{* *}$ - vs phases I and II; \# or \#\# vs group B.

\section{P130 The incidence of ARDS, interim results of the East Anglian ARDS Registry}

\section{JM Dixon and KEJ Gunning}

John Farman Intensive Care Unit, Box 93, Addenbrooke's NHS Trust, Hill's Road, Cambridge, CB2 2QQ, UK

Background: There is a wide variation in the reported incidence of acute respiratory distress syndrome (ARDS) due to the use of different diagnostic criteria. The publication by the American European Consensus Conference in 1994 of diagnostic criteria for ARDS has made comparisons of the incidence of ARDS more reliable. Only one study has looked at the incidence of ARDS in the UK. This was a retrospective survey in 1988 [1] that reported an incidence of 4.5/100 000 population/year.

Method: We report a prospective observational study of the incidence of ARDS in 10 ICUs in 9 hospitals covering a population of 1.89 million people over the age of 15 years. The consensus conference criteria were used to diagnose ARDS. Acute onset was defined as occurring within five days of onset of illness. A study co-ordinator in each participating ICU identified patients and there was regular feedback from the study organisers to ensure all cases were identified.

Results and discussion: Over the first 10 months of data collection, 68 patients met the criteria for the diagnosis of ARDS, giving an incidence of ARDS of 4.3/100 000 population/year. The mean age was 50.9 years. The mean length of stay on ICU of survivors was 16.9 days and 7.8 days for non-survivors, the intensive care mortality was 
$38 \%$. These interim results give an incidence of ARDS that is similar to that reported by the previous UK study and recent studies from other countries [2]. This figure would now appear to be a more accurate estimate of the incidence of ARDS in an unselected population. The study is ongoing and it is planned to follow up the survivors on a long-term basis.
References:

1. Webster NR, Cohen AT, Nunn JF: ARDS, how many cases in the UK? Anaesthesia 1988, 43:923-926.

2. Valta $P$, Uusaro $A$, Nunes $S$ et al:: Acute respiratory distress syndrome: frequency, clinical course and costs of care. Crit Care Med 1999, 27:2367-2374.

\section{P131 ARDS before and after the start of mechanical ventilation}

\section{A Anzueto, A Esteban, I Alía, L Brochard, T Stewart, F Frutos and MJ Tobin}

\section{International Mechanical Ventilation Study Group}

We determined if outcome differed in patients who developed ARDS before and after the institution of mechanical ventilation (MV). A prospective study of 5156 patients who underwent MV $>12 \mathrm{~h}$ was done in $361 \mathrm{ICU}$ from 20 countries. Among those patients, $261(5 \%)$ had ARDS at the onset of MV (prior-group), and $313(6 \%)$ developed ARDS $48 \mathrm{~h}$ or more after the onset of MV (after-group). The mean time from onset of MV to development of ARDS was $4 \pm 2$ days. In the after-group, MV was initiated for the following conditions: sepsis (19\%), aspiration (16\%), pneumonia (15\%), and trauma (15\%). MV parameters were similar in both groups. The patient characteristics and outcomes are shown in Table 1 (mean $\pm S D$ ).

Patients who developed ARDS after onset of MV were more likely to have ventilator associated pneumonia (38\% vs $26 \%, P<0.05)$ and coagulopathy $(31 \%$ vs $23 \%$,
Table 1

\begin{tabular}{lccc}
\hline & Prior-group & After-group & $P$ value \\
\hline Age (years) & $53 \pm 16$ & $57 \pm 17$ & 0.02 \\
SAPS II score & $47 \pm 17$ & $47 \pm 17$ & 0.74 \\
Duration MV (days) & $8 \pm 8$ & $12 \pm 9$ & 0.001 \\
In-ICU mortality & $54 \%$ & $65 \%$ & 0.01 \\
In-hospital mortality & $60 \%$ & $71 \%$ & 0.009 \\
\hline
\end{tabular}

$P=0.03$ ). In summary, patients who developed ARDS after the onset of mechanical ventilation were more likely to develop complications, required longer duration of ventilator support, and had higher ICU and hospital mortalities.

\section{P132 Prognostic factors in severe ARDS-patients}

\section{U Friess, E Münch, H Roth, M Quintel and K van Ackern}

Department of Anaesthesiology and Intensive Care, Universitätsklinikum Mannheim, University of Heidelberg, Germany

Aim of the study: To evaluate the prognostic value of different parameters on the outcome of conventionally treated ARDS-patients, admitted to the ICU as potential extra-corporal-membrane-oxygenation (ECMO) candidates.

Material and methods: Between 1995 and 1999, 115 patients with severe ARDS were transferred to the ICU of a University hospital as potential ECMO candidates. 22 patients underwent ECMO-therapy, 6 died soon after admission. 66 patients were treated with conventional therapy. In these 66 patients, we evaluated epidemiological characteristics, days in hospital before admission, days on the ventilator before admission, length of ICU-stay, days on the ventilator at the ICU, ventilator parameters (PEEP, peak airway pressure, mean airway pressure, $\mathrm{PaO}_{2} / \mathrm{FiO}_{2}$-ratio, compliance), biochemical parameters (haemoglobin, hematocrit, lactate, CRP, leukocytes, thrombocytes, creatinine), APACHE II and fluid balance on admission (1), 24 (2), 48 (3) and 72 (4) h after admission. Data are given as mean $\pm S D$. The Mann-Whitney U-test was used to identify significant differences between the groups.
Results: Forty-four of 66 patients survived, with an average Glasgow-outcome-scale of $3.88 \pm 0.44$. The mortality rate was $33 \%$ and mainly caused by MODS due to sepsis. None of the patients died of hypoxemia. The nonsurvivors were significantly older $(44.64 \pm 21.2$ years) than the survivors (32.39 \pm 12.2 years, $P<0.001)$, and had more concomitant diseases (28 vs 12), and a longer course of disease before hospitalisation ( $7.62 \pm 9.95$ vs $2.90 \pm 10.33$ days, $P<0.005)$. For hospital stay $(12.54 \pm 9.99$ vs $10.0 \pm 7.88$ days) or days on the ventilator before transfer (8.74 \pm 9.41 vs $6.98 \pm 6.48$ days) no difference could be shown. After admission the nonsurvivor group received more days of controlled ventilation (9.71 \pm 5.81 days) than the survivor group $(5.21 \pm 5.69$ days, $P<0.001)$, with regard to ICU stay no difference could be shown (19.09 \pm 14.99 vs $18.34 \pm 11.31$ days, ns). Significant differences between survivors and nonsurvivors are shown in Table 1.

No significant differences could be demonstrated for biochemical parameters, fluid balance, and compliance. 
Table 1

\begin{tabular}{|c|c|c|c|c|c|c|c|c|c|c|}
\hline \multirow[b]{2}{*}{ Time } & \multicolumn{2}{|c|}{ APACHE II } & \multicolumn{2}{|c|}{ PEEP $\left[\mathrm{cmH}_{2} \mathrm{O}\right]$} & \multicolumn{2}{|c|}{ PEAK $\left[\mathrm{cmH}_{2} \mathrm{O}\right]$} & \multicolumn{2}{|c|}{ MEAN $\left[\mathrm{cmH}_{2} \mathrm{O}\right]$} & \multicolumn{2}{|c|}{$\mathrm{PaO}_{2} / \mathrm{FiO}_{2}[\mathrm{mmHg}]$} \\
\hline & Alive & Dead & Alive & Dead & Alive & Dead & Alive & Dead & Alive & Dead \\
\hline 1 & $21.3 \pm 5$ & $27.0 \pm 4$ & $10 \pm 3$ & $11 \pm 3$ & $33 \pm 5$ & $35 \pm 5$ & $20 \pm 5$ & $22 \pm 5$ & $130 \pm 83$ & $83 \pm 35$ \\
\hline 2 & $17.4 \pm 5$ & $24 \pm 5$ & $10 \pm 3$ & $13 \pm 6$ & $31 \pm 6$ & $35 \pm 4$ & $18 \pm 5$ & $25 \pm 5$ & $166 \pm 74$ & $119 \pm 64$ \\
\hline 3 & $15.6 \pm 5$ & $24 \pm 5$ & $9 \pm 4$ & $14 \pm 4$ & $30 \pm 6$ & $35 \pm 4$ & $17 \pm 6$ & $24 \pm 5$ & $182 \pm 73$ & $129 \pm 56$ \\
\hline 4 & $13.3 \pm 7$ & $24 \pm 5$ & $8 \pm 5$ & $14 \pm 4$ & $31 \pm 4$ & $35 \pm 5$ & $17 \pm 6$ & $25 \pm 5$ & & \\
\hline
\end{tabular}

Conclusion: High APACHE II values, high PEEP, peak, and mean-airway-pressure levels and a low $\mathrm{PaO}_{2} / \mathrm{FiO}_{2}$ ratio are demonstrated to be of high prognostic value in a
non-ECMO treated severe ARDS population. None of the patients included in this survey died of hypoxemia, poor outcome was mainly determined by MODS due to sepsis.

P133 ${ }^{13} \mathrm{C}$-stable isotope analyses technique for ICU patients

\section{U Hölscher, T Gruchmann, Ch Goeters*, C Schulzki* and H Van Aken*}

Faculty of Applied Physics and Engineering, University of Applied Sciences, Hüfferstr. 27, D-48153 Münster; * Department of Anaesthesiology and Intensive Care Medicine, University of Münster, Münster, Germany

Introduction: By ${ }^{13} \mathrm{C}$ isotope spectroscopy, a noninvasive diagnostic tool for specific functions of different organs is available [1]. A ${ }^{13} \mathrm{C}$ labelled tracer is administered and is metabolised by a specific pathway to ${ }^{13} \mathrm{CO}_{2}$. The increase of exhaled ${ }^{13} \mathrm{CO}_{2}$ is analysed and gives an indication of a specific function of the organ under test. The availability of sensitive (resolution $0.3 \% 0$ ) and stable isotope-selective infra-red (IR) spectrometers has replaced expensive massspectrometers. The present IR analysers rely on two conditions: 1) on co-operating patients who collect breath samples into containers and; 2) on equilibrated test gas samples.

Methods: Online monitoring of ventilated ICU patients over a long period requires an automated collection of the exhaled gases and an equilibration of the $\mathrm{CO}_{2}$ content without compromising the lung ventilator set-up. The sample should only contain exhaled gases, a dilution would increase the necessary sensitivity of the analyser. A collection and mixing device has been developed that collects the exhaled gases and does not interfere with the safety and monitoring concept of the lung ventilator. The unit collects the sample gas from an Evita ventilator (Dräger) and supplies it to an Infra-Red Isotope-selective Spectrometer
(IRIS, Wagner) which has been modified to analyse a continuous gas stream. The expiratory concentrations of ${ }^{13} \mathrm{CO}_{2}$ and ${ }^{12} \mathrm{CO}_{2}$, the ratio of ${ }^{13} \mathrm{CO}_{2} /{ }^{12} \mathrm{CO}_{2}$ as well as the cumulated hourly percentage of the recovery of the administered ${ }^{13} \mathrm{C}$ label are determined by evaluation IRIS software (V2.3 1998, Wagner) and stored on a computer.

Results: The risk analysis and technical set-up have been reviewed and certified by the TÜV-Rheinland. The collection unit connecting the lung ventilator to the analyser has proven not to interfere with the functionality of the therapeutic device. The set-up was validated in volunteers and in ICU patients. Results of a first study determining the gastric emptying by isotope techniques will be discussed in parallel on this congress.

Conclusion: The stable IR isotope spectroscopy can be applied to ventilated patients. This allows a non-invasive focus e.g. on liver enzyme activities and on other organ specific problems.

\section{Reference:}

1. Ghoos $\mathrm{Y}:{ }^{13} \mathrm{CO}_{2}$ breath tests at the laboratory 'digestion-absorption'. University Hospital Gasthuisberg, Leuven 1996.

\section{P134 Metabolism in abdominal organs, as evaluated by microdialysis, in experimental severe acute} pancreatitis

P Ederoth, ZW Sun and R Andersson

Departments of Anesthesiology and Surgery, Lund University Hospital, S-221 85 Lund, Sweden

Introduction: Septic states might induce regional variations in metabolism. The aim of the present study was to evaluate if regional metabolic differences could be identi- fied in a model of severe acute pancreatitis in the rat, with the use of a microdialysis technique. 
Methods: Under full anesthesia, microdialysis probes were inserted in the parenchyma of the pancreas and liver and under the serosa of the small intestine. Microdialysate was collected every $10 \mathrm{~min}$. After a baseline period of 60 min, acute pancreatitis was induced by intraductal injection of $0.20-0.29 \mathrm{ml} 5 \%$ sodium taurodeoxycholate. The animals were studied for three $h$ after induction of pancreatitis after which they were killed. Arterial blood samples were taken every $60 \mathrm{~min}$. The microdialysis fluid and blood were analyzed for glucose and lactate. Six groups were studied with six animals in each group; Sham, Pancreatitis and four groups with treatment given $15 \mathrm{~min}$ after induction of acute pancreatitis (early treatment). Treatment administered included $\mathrm{N}$-acetylcystein (NAC; $200 \mathrm{mg} / \mathrm{kg}$ i.v), a platelet-activating factor (PAF) antagonist (lexipafant, $5 \mathrm{mg} / \mathrm{kg}$ ) and monoclonal antibodies against the adhesion molecules ICAM-1 (0.2 mg) and PECAM-1 (0.2 mg).

Results: The levels of lactate and glucose in pancreas and lactate in blood were higher $(P<0.05)$ in the pancreatitis group compared with the sham group. The increases of glucose and lactate in the pancreas were higher than in the blood, demonstrating an earlier and stronger change in metabolism in the pancreas as compared with the rest of the body. This finding was higher $(P<0.05)$ in the pancreatitis group than in the sham group. There were no clear differences between the various pancreatitis groups, with or without treatment. and no differences in between the treatment groups. Within the sham group, glucose showed no regional difference, but there was a significant increase in the pancreas in pancreatitis animals as compared to the liver and intestine. Regarding lactate, there were differences between the pancreas, liver and intestine in both groups with higher levels seen in the pancreas.

Conclusion: An increase in pancreatic lactate concentrations in pancreatitis animals was seen and this increase seemed to have its origin in the pancreas and not in the whole body. Similar changes were also found for glucose. Early treatment, as described earlier, had no obvious effect on these parameters. The results imply that microdialysis could be of potential future value in monitoring metabolic locoregional differences in critical illness.

\section{P135 Acute necrotizing pancreatitis - histological study focusing on necrosis and apoptosis}

\section{Y Haraguchi, Y Tomoyasu, N Shindo, J Inoue, Y Itoh, M Hoshino, S Hasegawa, T Ishihara and T Nagata}

National Hospital Tokyo Disaster Medical Center, Tokyo, Japan

Acute necrotizing pancreatitis is histologically studied focusing on the degree and extent of necrosis and apoptosis.

Materials and methods: Evaluation was done by lightmicroscopic examination (Hematoxylin-Eosin stain) and electron-microscopic examination. In order to judge the existence of apoptosis, the TUNEL method was applied.

Only exocrine tissue was surveyed. The number of specimens is three, which were obtained by distal pancreatectomy in the early phase of acute necrotizing pancreatitis because they resisted intensive care. The normal control was one specimen in which 1) negative control and; 2) positive control (TACS-Nuclease-treated control) were used.

Results: Pancreatic tissue was divided into three areas by light- and electron- microscopy before TUNEL: 1) micro- scopically normal tissue; 2) microscopically apparently necrotized area; 3 ) minimal or almost normal tissue by the light microscope, although electron-microscopically mild or moderate destructive change of the cytoplasm and nuclear structure was apparent. The TUNEL method revealed the existence of apoptosis in both the the necrotized area (2) and in the minimally destroyed area lightmicroscopically (3).

Discussion and conclusions: From our study, the existence of apoptosis in acute necrotizing pancreatitis is thought to exist. Several important themes seem to be pointed out: 1) new classification of acute pancreatitis, including apoptosis may be necessary; 2) apoptosis will influence the prognosis or mortality of necrotizing pancreatitis, hitherto believed to depend on the ratio of necrosis; 3) the criteria of severity should be revised.

\section{P136 Inflammatory response, prognostic scores and mortality in acute pancreatitis}

MV De la Torre Prados, MA Herce Muñoz*, A Soler García, C Reina Artacho, A García Alcántara, A Poullet Brea and M Morell Ocaña*

Servicio Medicina Intensiva, *Servicio de Bioquímica, H.U. Virgen Victoria, 29010-Málaga, Spain

Objective: The object of the study was to relate, in the first $72 \mathrm{~h}$ following hospital admission, biochemical markers of inflammatory response and prognostic scores to mortality in acute pancreatitis (AP).
Materials and methods: For a year (November 1997 to October 1998) a prospective study was made consecutively of 30 patients admitted to the Emergency Department where AP was diagnosed; $83.3 \%$ were in the Intensive Care Unit (ICU) with three or more Ranson criteria and $24 \mathrm{~h}$ APACHE score $>8$. At 12, 36, 48 and $72 \mathrm{~h}$ 
Table

\begin{tabular}{|c|c|c|c|c|c|c|}
\hline \multirow[b]{2}{*}{ Variables } & \multicolumn{2}{|c|}{$12-30 \mathrm{~h}$} & \multicolumn{2}{|c|}{$30-48 \mathrm{~h}$} & \multicolumn{2}{|c|}{$48-72 \mathrm{~h}$} \\
\hline & NS & $S$ & NS & $S$ & NS & $S$ \\
\hline Elastase $\mu \mathrm{g} / \mathrm{l}$ & $221(17)$ & $213(29)$ & $210(10)$ & $190(34)$ & $162(43)$ & $163(45)$ \\
\hline CPR mg/dl & $11(7)$ & $10(8)$ & *33 (7) & *19 (12) & $29(15)$ & $23(12)$ \\
\hline IL-6 pg/ml & $123(67)$ & $138(80)$ & $348(97)$ & $301(85)$ & $184(86)$ & $182(82)$ \\
\hline IL-8 pg/ml & *107 (92) & *55 (43) & *87 (62) & *40 (31) & $28(19)$ & $52(32)$ \\
\hline \multirow[t]{2}{*}{ TNF pg/ml } & $20(12)$ & $13(5)$ & *45 (32) & ${ }^{*} 13(6)$ & *29 (10) & *15 (10) \\
\hline & \multicolumn{2}{|c|}{$24 \mathrm{~h}$} & \multicolumn{2}{|c|}{$48 \mathrm{~h}$} & \multicolumn{2}{|c|}{$72 \mathrm{~h}$} \\
\hline APACHE II & $16(5)$ & $10(6)$ & *15(5) & ${ }^{\star} 8.8(6)$ & $13(5)$ & $8(6)$ \\
\hline Ranson & $3.2(0.4)$ & $3.1(0.9)$ & *7.2(1.6) & *5.3(2.3) & & \\
\hline
\end{tabular}

Values are shown as means(SD). NS=Nonsurvival, $S=$ Survival. $\left(^{*}\right)$ T-test, $P<0.05$.

biochemical parameters were measured: elastase, C-reactive protein (CRP), interleukin (IL)-6, IL-8 and Tumor Necrosis Factor (TNF). At the same time 24, 48 and $72 \mathrm{~h}$, APACHE scores and 24 and $48 \mathrm{~h}$ Ranson scores were assessed.

Results: Thirty patients with a mean age of 62.7 (13.5) years were studied. $60 \%$ were women and $80 \%$ had biliary etiology. The median interval from pain until hospital admission was 16.6 h. $67 \%$ showed C or D grade Balthazar Scale. $60 \%$ had surgery at 38 days median period.
$56.7 \%$ had some complications and the hospital mortality was $16.7 \%$. In relation to mortality there was a significant difference (T-test) in the number of dysfunctional organs $3.8(1.3)$ vs 1.7(1.2) and in the Multiple Organ Dysfunction (MOD) score 8.8 (6) vs 3 (2.2).

Conclusions: In the nonsurvival group, IL-8 increased significantly in the first $30 \mathrm{~h}$ and CPR, IL-8 and TNF between 30 and $48 \mathrm{~h}$. Between 48-72 h, TNF continued to increase. There were differences in APACHE and Ranson Scores, but these were only significant at $48 \mathrm{~h}$.

\section{P137 Indocyanine green clearance monitoring during liver transplant for fulminant hepatic failure: preliminary results}

G Della Rocca, MG Costa, S Monaco, D Romboli, S Costini and P Pietropaoloi

Istituto di Anestesiologia, University of Rome "La Sapienza", Viale del Policlinico n¹55-00161 Rome, Italy

Introduction: The COLD System (Pulsion Medical System, Munich, Germany) allows measurements of cardiac index (Cl), intrathoracic blood volume (ITBV), extravascular lung water (EVLW) and liver function through the plasma disappearance rate of indocyanine green dye (ICG) (PDRdy n.v.=20-30\% min).

Methods: Six patients (6F), with a mean age of $42.2 \pm 11.7$ years and with a mean BSA of $1.7 \pm 0.1 \mathrm{~m}^{2}$, affected by fulminant hepatic failure (FHF), undergoing orthotopic liver transplantation (OLT) were studied with the COLD System to evaluate PDRdy as a marker of native and transplanted liver function and volumetric data. After anesthesia induction, a pulmonary artery catheter (Intellicath CCO; Baxter, Irvine, CA, USA) was placed via the right subclavian vein and a $3 F r$ thermistor-tipped fiberoptic catheter for thermal dye dilution was inserted into the descending aorta via a 4Fr introducer sheath in the femoral artery and connected to the COLD-Z021. All data were collected during the following steps: after anesthesia induction $(A)$, during anhepatic phase $(B)$, after graft reperfusion (C), at the end of surgery (D) and $6 \mathrm{~h}$ after the end of surgery $(E)$.

Results: Results are reported in the table. Four patients had a rapid weaning, were extubated, discharged from ICU and are still enjoying good health. Two patients, despite a good liver function recovery, died with MOF.

Conclusion: The evaluation of cardiac preload is a useful tool to guide fluid replacement and vasoactive drug admin-

Table

\begin{tabular}{lccccc}
\hline & $\mathrm{A}$ & $\mathrm{B}$ & $\mathrm{C}$ & $\mathrm{D}$ & $\mathrm{E}$ \\
\hline Cl & $5 \pm 1.4$ & $3.4 \pm 0.9$ & $4.2 \pm 1.4$ & $4.1 \pm 0.8$ & $4.4 \pm 0.4$ \\
ITBVI & $856 \pm 101$ & $692 \pm 124$ & $838 \pm 204$ & $818 \pm 116$ & $764 \pm 123$ \\
EVLWI & $6.9 \pm 3.3$ & $9 \pm 6.5$ & $7.9 \pm 4.7$ & $9.2 \pm 6.7$ & $8.2 \pm 6.0$ \\
PDRdy & $4.2 \pm 3.1$ & $3.2 \pm 2.1$ & $21.6 \pm 6.6$ & $19.4 \pm 6.4$ & $18.3 \pm 2.7$ \\
\hline
\end{tabular}


istration during anesthesia for a major surgical procedure such as OLT. In selected cases with FHF, PDRdy monitor- ing allows one to obtain an accurate bedside evaluation of graft function recovery.

P138 Clinical and economic study of the use of somatostatin 14 AA (amino-acids) in the treatment of enterocutaneous and pancreatic fistulas

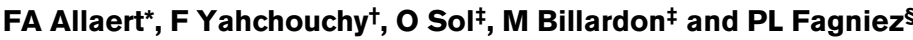 \\ ${ }^{*}$ Centre d'Evaluation et de Normalisation, $\mathrm{CHU}$ du Bocage, 21000 Dijon; ${ }^{+}$Chirurgie générale, Hôpital de Poissy, 78303 Poissy; \\ ‡UCB Pharma, 21 Rue de Neuilly, 92003 Nanterre; §Hôpital Henri Mondor, 94010 Créteil, France
}

Purposes of the study: To describe the use of somatostatin 14 AA (amino-acids) by patients with post-surgical enterocutaneous and/or pancreatic fistulas, its clinical results, and the analysis of the economic benefits resulting from such treatment.

Material and methods: Nature of the study: open medico-economic observational study. Follow-up period of the patients: from somatostatin $14 \mathrm{AA}$ treatment initiation to the closure of the fistula (fistula output $\leq 10 \mathrm{ml} / 24 \mathrm{~h}$ ) or to a maximum of 28 days. Criteria of selection: presence of one or more entero-cutaneous and/or pancreatic postsurgical fistulas. Evaluation criteria: 1) closure rate and time to closure of the fistulas; 2 ) hospital expenses.

Results: Eighty patients were included in the study. The average age of the patients was $58.4 \pm 16.6$ years (mean $\pm S D) .87 .7 \%(n=64)$ of the patients had single fistula and $12.3 \%(n=9)$ had multiple fistulas. $37.0 \%(n=27)$ were ileal, $17.8 \%(n=13)$ jejunal and $45.2 \%(n=33)$ pancreatic. $60.8 \%$ fistulas were closed after $15.2 \pm 7.6$ days and $11.5 \pm 5.1$ days of treatment. The percentage of closure is comparable whatever the clinical characteristics (age, sex, associated pathology) of the patients, the conditions of the surgical operation (first intervention or re-intervention, regular or emergency surgery) and the type of fistula (ASA classification, morphology, topography). The percentage of closure varies significantly according to the response at $48 \mathrm{~h}$. Respectively, among the patients who have a decrease of $50 \%$ and $70 \%$ of their fistula output at $48 \mathrm{~h}$, $80 \%(n=32)$ and $91.7 \%(n=22)$ have an output less than $10 \mathrm{ml} / 24 \mathrm{~h}$ at the end of the study. The rate of closure of the fistula does not differ from those described in the literature for treatments without somatostatin $14 \mathrm{AA}$ but the time of closure is halved from 31 days to 15.2 days. At comparable therapeutic success rate, the use of somatostatin $14 \mathrm{AA}$ provides greater comfort for the patient and an important decrease in costs (30\%) arising from the reduction in the duration of the hospital stay.

Conclusion: The use of somatostatin 14 AA shortens the time of closure of the enterocutaneous and pancreatic fistulas and allows an important reduction of the hospital costs. The evaluation of the therapeutic response $48 \mathrm{~h}$ after the beginning of the treatment is a fundamental criterion for optimising the use of this drug.

\title{
P139 Decrease in urine output during mesenteric traction syndrome is an early predictor of multiorgan dysfunction
}

P Garcia-Saura, A Marquez, C Serrano, J Prieto, A Maldonado, MS Serrano-Atero and F Peramo

Unidad de Reanimacion, Departamento de Anestesiologia, Reanimación y Terapia del Dolor, Hospital Clinico Universitario de Granada, Spain

Introduction: Six retrospective case-control studies showed that the only difference between those patients who underwent elective infrarenal aortic aneurysm repair (IAAR) developing mesenteric traction syndrome (MTS) in the operation room (OR) and multiorgan dysfunction syndrome (MODS) during their ICU stay, was the decrease in urine output before aortic cross-clamping. Two of them presented the socalled 'hepatohemorrhagic syndrome' (HHS) [1] characterised by the association of disseminated intravascular coagulation (DIC) and acute ischemic hepatitis (AlH).

Methods: Data from six patients with MTS was obtained from the anaesthesia records (1995-1998) and clinical evolution during their ICU stay.

Results: After the placement of the intestinal traction device, all patients developed MTS (facial flushing, reduced mean arterial pressure (MAP) and systemic vascular resistance (SVR) with increased heart rate $(\mathrm{HR})$ and Cardiac Index $(\mathrm{Cl})$. They were treated with volume replacement until they reached the previous MAP before cross-clamping. Diuresis showed an increase that remained elevated during the intervention in all the patients but two. These two developed DIC intra-operatively, with bleeding before unclamping and posterior MODS. We believe that the initial release of PGI2, as the main product triggering the MTS, is also responsible for the increase in diuresis, but dysregulation of the renal function by an unknown mechanism [2] in the context of the prostanoid disbalance, would explain the two cases of DIC and posterior MODS in the two patients which, after a little increase in urine output, suddenly without any explanation turned to fall [3]. 


\section{Figure}

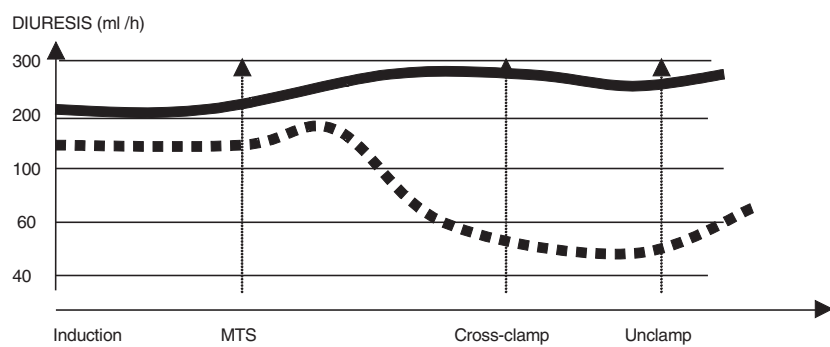

Conclusions: First, although it has been postulated that HHS can not be explained by the operative course before the release of the aortic cross-clamp, we found a close cause-effect relationship between the presence, or not, of MTS during the anaesthesia period, and the posterior course in which urine output fell. Second, we also think that this syndrome (at least the two HHS we have seen) would be included in the context of MODS. Third, in this setting, the kidneys are the first organs in dysfunction by unknown mechanism, and this is due to the impaired renal response to the disbalance of prostanoids.

\section{References:}

1. Levy PJ, Tabares AH, Olin JW, et al: Disseminated intravascular coagulation associated with acute ischemic hepatitis after elective aortic aneurysm repair: comparative analysis of 10 cases. $J$ Cardiothoracic Vascular Anaesthesia 1997, 2:141-148.

2. Gelman S: The pathophysiology of aortic cross-clamping and unclamping. Anesthesiology 1995, 82(4).

3. Maldonado AJ, Costela JL, Serrano-Atero MS, et al: Novedades anestésicas en angiología y cirugía vascular. In Novedades Farmacológicas en las Vasculopatias. Edited by Ros Die E. Barcelona: J Uriach and Cia. SA, Barcelona.

\section{P140 Increased intestinal permeability accompanies the development of sepsis in ICU patients}

M Hersch, I Skorochod, L Kanter, M Gurevitz and R Goldstein

Department of Anesthesiology Intensive care and Medicine, Shaare Zedek Medical Center, Jerusalem, Israel

Introduction: The concept of bacterial/toxin translocation from the gut as the source of sepsis is well recognized. Continuous monitoring of gut permeability in correlation with clinical signs of sepsis is poorly documented. We measured daily intestinal permeability of patients admitted to the ICU and correlated it with signs of sepsis.

Methods: Design: prospective, observational study. Population: twelve consecutive patients admitted to the ICU with an expected admission of three days or more. Three patients were septic on admission and nine were not. Six out of the nonseptic group developed signs of sepsis during their ICU stay. Permeability measurement: each of the 12 patients received $5 \mathrm{~g}$ of Mannitol and $10 \mathrm{~g}$ of Lactulose daily (from ICU admission up to seven days), through their NG tube. Urine was collected every $24 \mathrm{~h}$, and an aliquot of $40 \mathrm{ml}$ was kept at $-20^{\circ} \mathrm{C}$ for subsequent high pressure liquid chromatography, to measure the secretion of Mannitol and Lactulose. The ratio of Lactulose/Mannitol (L/M) was calculated. Signs of sepsis (according to Bone's criteria: Ann Int Med, 1991) were recorded daily.

Results: A total of nine septic patients showed a significant increase of $\mathrm{L} / \mathrm{M}$ compared to nine non-septic patients $(21.2 \pm 0.3$ vs $4.3 \pm 0.4, P<0.05)$. The six patients who developed sepsis during their ICU stay showed a significant rise of $\mathrm{L} / \mathrm{M}$ while becoming septic $(28.7 \pm 0.2$ during sepsis, vs $4.8 \pm 0.3$ when not septic, $P<0.05)$.

Conclusions: Increased intestinal permeability is associated with clinical signs of sepsis, thus substantiating the role of gut permeability as a major defect in the evolvement of sepsis and its sequelae.

\section{P141 Hypocapnic and normocapnic ventilation in hepatic encephalopathy: a comparision}

AK Baronia, CK Pandey and N Mathur

SGPGIMS, Lucknow, 226014 , India

Purpose: Encephalopathy with raised intracranial pressure is the major cause of death in hepatic failure [1]. We investigated role of arterial hypocapnia, achieved by hyperventilation, in the management of hepatic encephalopathy.

Methods: In a prospective study of twenty-two patients, with fulminant hepatic failure with grade IV encephalopathy receiving mechanical ventilation, two groups of twelve (group I) and ten (group II) patients had $\mathrm{PaCO}_{2}$ in the range of $25-30 \mathrm{mmHg}$ (group I) and $35-40 \mathrm{mmHg}$ (group II). Tidal volume was kept in the range of $8-10 \mathrm{ml}$ per $\mathrm{kg}$ and respiratory rate in the range of $12-20$ per min.

Results: The demographic data was comparable in both the groups. The shortest duration of recovery was $8 \mathrm{~h}$ in group I and $30 \mathrm{~h}$ in group II. Survival was $20 \%$ group I and $41.66 \%$ group II. No adverse effects, secondary to hypocapnia, were noticed in group I. 
Conclusion: Improved survival in the normocapnic group may indicate that normocapnic ventilation may be preferred over hypocapnic ventilation, particularly if the patients of hepatic encephalopathy are being managed without cerebral perfusion pressure and ICP monitoring.

\section{Reference:}

1. Ede RJ, Williams R: Semin Liver Dis 1986, 6:107-118.

\section{P142 Emergency department evaluation of orthotopic liver transplant recipients}

G Couto, S Marum, J Oliveira, M Rui Silva and J Palmeiro Ribeiro

Unidade de Cuidados Intensivos do Hospital de Curry Cabral, Lisboa, Portugal

Introduction: The intensive care unit (ICU) has an important role in the early management of orthotopic liver transplantation (OLT) recipients.

Patients and methods: We reviewed the records of the 83 consecutive patients (91 procedures) who underwent OLT from September 1992 to June 1999 in our centre, collecting sample characteristics, reviewing the early complications after OLT and the changes in medical care of these patients over the past seven years.

Results: Most frequent indications for primary OLT and for retransplantation were familial amyloidotic polyneuropathy $(37.3 \%)$ and hepatic artery thrombosis (75\%), respectively. The most challenging complications seen were acute renal failure (31.3\%), postoperative bleeding (15.6\%), infections (9.6\%), acute cellular rejection $(9.6 \%)$ and warm ischemia $(8.4 \%)$, with lethal primary nonfunction ensuing it in three cases, the most common cause of death in ICU. There was considerable reduction in operative time $(7.1 \pm 1.36$ vs $5.8 \pm 0.83 \mathrm{~h} ; P<0.001)$, blood transfusions requirement $(8.1 \pm 4.6$ vs $4.9 \pm 2.7$ units; $P<0.01)$, ventilation time in the routine patient ( $51 \pm 30$ vs $30 \pm 30 \mathrm{~h} ; P<0.05)$ and in ICU length of stay $(6.2 \pm 2.7$ vs $3.9 \pm 2.5$ days; $P<0.002)$ between the two time periods in which we equally divided our sample (92-96 and 97-99) with a sensible reduction in ICU morbidity (48\% vs $24 \%$ of moderate-to-severe complications; $P<0.02)$.

Discussion and conclusions: The reduction in procedure and ICU invasiveness throughout the years allowed for a reduction in morbidity and may confirm that appropriate medical care can overcome the adverse influences of many of the negative predictor factors suggested to influence OLT recipients outcome.

\section{P143 Timing and incidence density of upper gastrointestinal bleeding acquired by critically ill children} M Chaïbou, M Tucci, M A Dugas, CA Farrell and J Lacroix Pediatric Intensive Care Unit, Department of Pediatrics, Sainte-Justine Hospital, Université de Montréal, Montréal, Québec, Canada

Objectives: To determine in critically ill children: 1) when upper gastrointestinal bleeding (UGIB) and clinically significant UGIB (CS-UGIB) occur; 2) the mean incidence density and the cumulative incidence of UGIB and CSUGIB.

Design: Prospective epidemiological study.

Site: Multidisciplinary pediatric intensive care unit (PICU) in a tertiary care teaching hospital.

Methods: UGIB was considered to be present if hematemesis occurred or if blood was present in the gastric tube. UGIB was qualified as clinically significant if 2 out of 3 experts independently concluded that at least 1 of 6 complications (transfusion, decreased hemoglobin concentration, hypotension, surgery, multiple organ system failure or death) was attributable to it. The mean time from PICU admission to onset of UGIB or CS-UGIB was obtained by the summation of the number of events multiplied by duration of PICU stay for each event, then divided by the total number of events. The mean incidence density was calculated by dividing the total number of patients with UGIB or CS-UGIB by the cumulative number of patient-days.

Results: The cohort included 1006 consecutive patients. 103 UGIB (10.2\%) were diagnosed, including 16 CSUGIB (1.6\%). The mean time from PICU admission to UGIB and CS-UGIB onset was 4.5 days $(95 \% \mathrm{Cl}$ : 1.4-7.6) and 3.9 days (95\% $\mathrm{Cl}$ : 1.4-6.4) respectively. The mean incidence density was 24.6/10 000 patientdays for UGIB and 3.8/10 000 patient-days for CS-UGIB. The estimation of the risk of bleeding by actuarial method indicates that the probability of occurrence of UGIB and CS-UGIB was highest on the second day of PICU stay: $4.7 \%$ and $1.2 \%$ respectively.

Conclusion: UGIB and CS-UGIB occur soon after admission to PICU. Prophylaxis to prevent CS-UGIB should be given early to patients at risk of developing this complication. 


\section{P144 Does metoclopramide prevent bacterial translocation (BT)?}

\section{F Cansizoglu, M Tulunay, M Oral, Z Alanoglu, A Karaaslan, D Gerceker and I Palabiyikoglu}

Ankara University Medical Faculty, Anesthesiology and Reanimation Department, Sihhiye, Ankara, Turkey

One of the mechanisms causing BT is impaired host defense. Metoclopramide (M) restores the depressed immune function after hemorrhage [1]. To our knowledge, there is no study investigating the effect of $M$ on BT.

Procedures: We investigated the administration of $M$ on BT in a dog model of ischemia-reperfusion (IR) injury induced by thoracic aortic cross-clamping and declamping. Twenty-two mongrel dogs were randomized into three groups: sham-operated group $(n=7)$ (without cross-clamping), $M$ group $(n=8)$ and placebo group $(n=7)$. Placebo and $M$ group received placebo and $M(0.15 \mathrm{mg} / \mathrm{kg}$ iv), respectively, before cross-clamping. During $45 \mathrm{~min}$ of ischemia and 30 min reperfusion arterial blood gases and hemodynamic data were continuously recorded. $72 \mathrm{~h}$ later dogs were re-operated on, and peritoneal swabs, blood samples and specimens from duodenum, jejunum, ileum, colon, mesenteric lymph node (MNL), heart, spleen, kidney, liver and lung were obtained for bacteriological analysis. Blood samples were also obtained before the first operation.
Results: No dogs in the sham group showed BT. Two ischemia-reperfusion groups were found to have BT in various organs; however, M prevented BT to the $\operatorname{MLN}\left(\chi^{2}\right)$ without producing any hemodynamic changes (MannWhitney U-test, Kruskal Wallis one way ANOVA, Friedman two way ANOVA) organs $\left(\chi^{2}\right)$. Both gram ( $)$ and gram $(+)$ microorganisms translocated. One dog in the placebo group and two dogs in $\mathrm{M}$ group developed bacteremia after the first operation.

Conclusion: M prevents BT to the MLN. Whether higher doses of $\mathrm{M}$ prevent $\mathrm{BT}$ and the clinical importance of these results requires further studies.

\section{Reference:}

1. Zellweger R, Wichmann MW, Ayala A, Chaudry IH: Metoclopramide: a novel and safe immunomodulating agent for restoring the depressed macrophage immune function after hemorrhage. $J$ Trauma 1998, 44:70-77.

\section{P145 Is redistribution of microcirculatory blood flow within the small intestinal wall the cause of prolonged paralytic ileus in the critically ill?}

L Hiltebrand, V Krejci, D Erni*, A Banic ${ }^{*}$ and GH Sigurdsson

Autoregulation of microcirculatory blood flow (MBF) and redistribution of flow within the intestinal wall (from the muscularis to the mucosa) are known mechanisms to maintain $\mathrm{O}_{2}$ delivery in the gut during hypovolemia. During sepsis, however, autoregulation of blood flow is impaired [1]. The aim of this study was to monitor the distribution of MBF within the intestinal wall in different parts of the gastrointestinal tract during the development of septic shock.

Materials and methods: We measured systemic (Cl), regional (mesenteric artery; SMA) and microcirculatory blood flow (MBF) in 11 sedated and ventilated pigs. MBF was measured with multichannel laser Doppler flowmetry in the gastric, jejunal, and colonic mucosa and the corresponding muscularis layers. Septic shock was induced by faecal peritonitis. After 240 min, i.v. fluids were administered to alter hypodynamic shock to hyperdynamic septic shock.

Results and discussion: During the first 240 min (hypodynamic shock) $\mathrm{Cl}, \mathrm{SMA}$ and MBF in the stomach mucosa decreased by $50 \%$ (Figs 1 and 2), while MBF in the jejunal and colonic mucosa remained virtually unchanged (Figs 3 and 4). In the muscularis of the jejunum and colon, on the other hand, MBF decreased significantly more than
$\mathrm{Cl}$ and SMA (Figs 2 and 4). Administration of i.v. fluids at 240 min resulted in a significant increase (above baseline) in $\mathrm{Cl}$ and SMA (Fig. 1) as well as in MBF in the mucosa of the stomach, jejunum and colon. The 'overshooting' increase in MBF in the jejunal and colonic mucosa, indicates that there was some hypoperfusion present in the mucosa during the hypodynamic phase despite maintained blood flow. This is supported by a significant decrease in jejunal pHi (Fig. 5) during the same time period. There was virtually no increase in MBF in the jejunal and colonic muscularis after fluid administration indicating a prolonged, perhaps endothelin induced, hypoperfusion.

Conclusion: It was concluded that MBF in the mucosa of the jejunum and colon remained unchanged, despite a $50 \%$ decrease in systemic and regional flows, suggesting a largely intact autoregulation during septic shock. MBF in the jejunal and colonic muscularis decreased significantly more than systemic and regional flows, suggesting that there is an active mechanism to maintain adequate blood flow to the mucosa during septic shock. Redistribution of blood flow from the muscularis to the mucosa causes severe hypoperfusion of the muscularis which contributes to intestinal atony frequently observed in critically ill patients. 
Figures 1-5

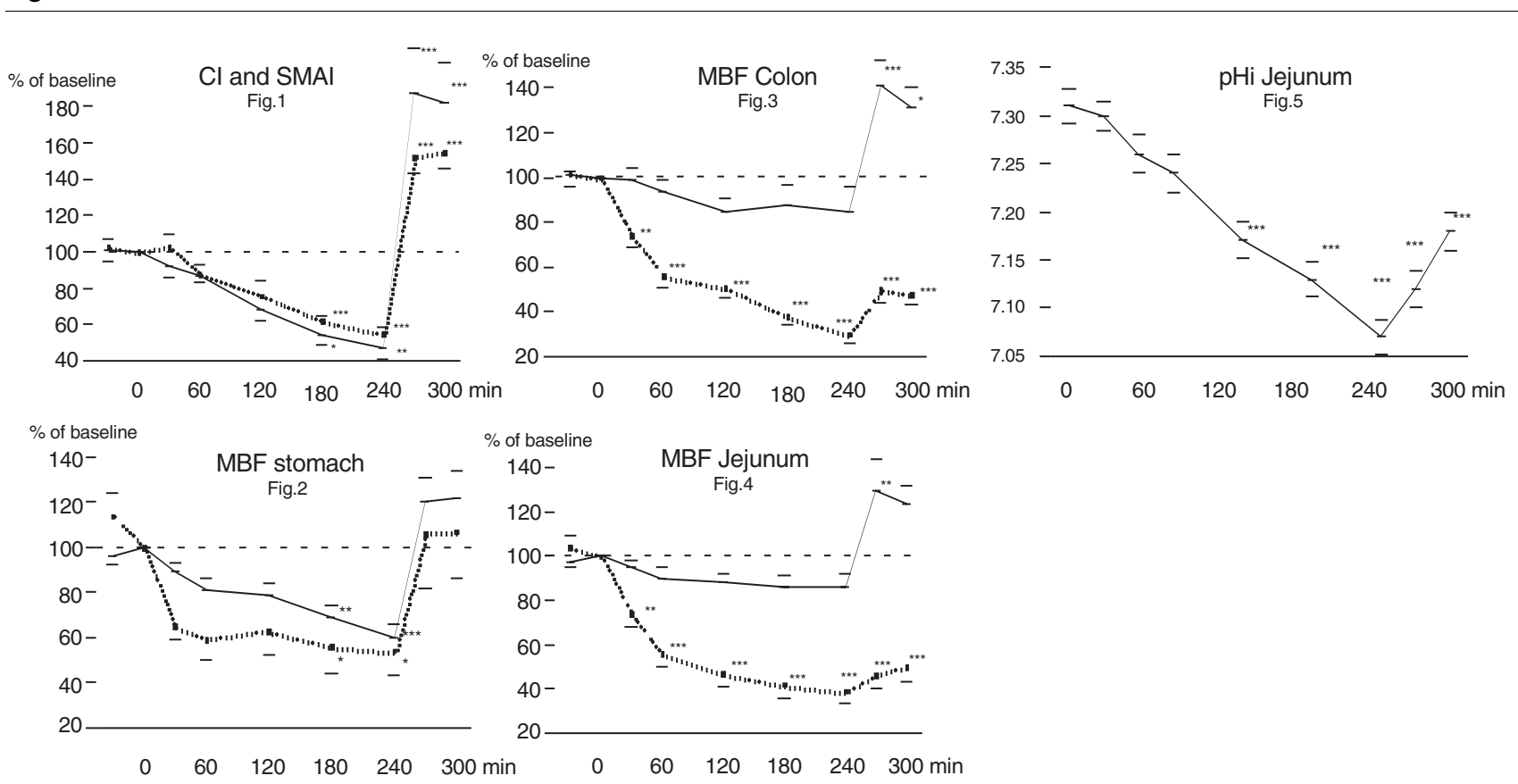

Onset of peritonitis at $0 \mathrm{~min}$ and i.v. fluid administration at $240 \mathrm{~min}$. Data is presented as mean \pm SEM. Statistics: ANOVA for repeated mesurements. $P<0.05$ was considered statistically significant.

\section{P146 Ileal microcirculation and mucosal acidosis during hyperdynamic porcine endotoxemia}

\section{Tugtekin, M Matejovic*, A Stehr, M Theisen, F Ploner ${ }^{\star}$, K Träger, M Georgieff and P Radermacher}

Department of Anesthesia, Univ. Hospital, D-89073 Ulm, *Department of Intensive Care, Charles-University, CZ-30460 Plzen; ${ }^{+}$Department of Surgery, Univ. Hospital, D-93053 Regensburg; ${ }^{\ddagger}$ Department of Anesthesia, District Hospital, I-39042 Brixen

Introduction: The tonometric determination of the arterialmucosal $\mathrm{PCO}_{2}$-gap $\left(\Delta \mathrm{a}-\mathrm{rPCO} \mathrm{PC}_{2}\right)$ is used to monitor adequacy of gastrointestinal perfusion. As $\mathrm{PrCO}_{2}$ also integrates other phenomena [1], we analyzed the influence of the intestinal villus microcirculation on increased $\triangle \mathrm{a}-\mathrm{rPCO}$ during long-term hyperdynamic porcine endotoxemia.

Material and methods: Anesthetized and ventilated pigs received continuous i.v. endotoxin $(E T X, n=12)$ for $24 \mathrm{~h}$ or placebo (Sham, $n=6$ ). Hydroxyethylstarch was infused to maintain MAP >65 mmHg together with a sustained increase in cardiac output [2]. Before the start of ETX (0 h), as well as 12 and $24 \mathrm{~h}$ afterwards, portal venous blood flow (Qpv, ultrasound flow probes) and lactate/pyruvateratios ( $\mathrm{L} / \mathrm{P} \mathrm{pv}$ ), ileal mucosal $\Delta \mathrm{a}-\mathrm{r} \mathrm{PCO}_{2}$ (fiberoptic sensor) and bowel wall capillary $\mathrm{Hb}_{2} \mathrm{O}_{2}$-saturation $\left(\mathrm{Hb}-\mathrm{O}_{2}\right.$ cap, remission spectrophotometry) were assessed together with intravital video records of the ileal mucosal microcir-

Table

\begin{tabular}{|c|c|c|c|c|}
\hline & & O h ETX & 12 h ETXk & 24 h ETX \\
\hline Opv ml/kgxmin & $\begin{array}{l}\text { ETX } \\
\text { Sham }\end{array}$ & $\begin{array}{l}24(21,27) \\
22(18,25)\end{array}$ & $\begin{array}{l}27(22,31) \\
26(25,29)\end{array}$ & $\begin{array}{l}26(20,31) \\
25(22,29)\end{array}$ \\
\hline $\mathrm{Hb}-\mathrm{O}_{2}$ cap \% ( \pm S.D.) & $\begin{array}{l}\text { ETX } \\
\text { Sham }\end{array}$ & $\begin{array}{l}51 \pm 16 \\
53 \pm 15\end{array}$ & $\begin{array}{l}53 \pm 16 \\
53 \pm 10\end{array}$ & $\begin{array}{l}53 \pm 11 \\
44 \pm 13\end{array}$ \\
\hline$\Delta \mathrm{a}-\mathrm{rPCO} \mathrm{CO}_{2} \mathrm{mmHg}$ & $\begin{array}{l}\text { ETX } \\
\text { Sham }\end{array}$ & $\begin{array}{l}15(8,17) \\
14(9,17)\end{array}$ & $\begin{array}{l}18(15,26) \# \\
14(10,16)^{\S}\end{array}$ & $\begin{array}{c}22(16,33) \# \\
13(10,20)\end{array}$ \\
\hline $\mathrm{L} / \mathrm{P} \mathrm{pv}$ & $\begin{array}{l}\text { ETX } \\
\text { Sham }\end{array}$ & $\begin{array}{l}14(13,17) \\
11(10,13)\end{array}$ & $\begin{array}{c}19(17,22) \# \\
13(8,19)\end{array}$ & $\begin{array}{c}26(21,31) \# \\
14(10,25)\end{array}$ \\
\hline n (Villi) perfused/not perfused & $\begin{array}{l}\text { ETX } \\
\text { Sham }\end{array}$ & $\begin{array}{c}60(48 ; 74) / 0(0 ; 0) \\
73(66 ; 130) / 0(0 ; 0)\end{array}$ & $\begin{array}{c}33(6 ; 52) / 28(18 ; 42) \# \S \\
62(54 ; 82) / 6(2 ; 11)^{\S}\end{array}$ & $\begin{array}{c}32(23 ; 38) / 22(10 ; 35) \# \S \\
55(27 ; 100) / 0(0 ; 4)^{\S}\end{array}$ \\
\hline
\end{tabular}


culation (number of perfused/not-perfused villi) by orthogonal polarization-spectrometry $\left(\right.$ Cytoscan $\left.{ }^{\mathrm{TM}}\right)$ [3] obtained via an ileostomy.

Results: See Table. Median(25/75\%); \#P<0.05 vs $0 \mathrm{~h}$ (Friedman-Anova); ${ }^{\circledR} P<0.05$ ETX vs Sham (Mann-Whitney).

Conclusion: Taking into account the unchanged portal venous blood flow, the progressive increase in $\triangle \mathrm{a}-\mathrm{rPCO}{ }_{2}$ is mainly due to the heterogeneity of capillary villus perfu- sion. We can only speculate about the putative additional influence of a disturbed intracellular $\mathrm{O}_{2}$-utilisation.

Acknowledgement: Supported by *ESICM, Deutsche Forschungsgemein schaft and FProvinz Bozen-Südtirol (Italy). Cytoscan ${ }^{\mathrm{TM}}$ is a trademark of Cytometrics Inc., Philadelphia, PA.

References:

1. Brinkmann et al.: Intensive Care Med 1998, 24:542.

2. Träger et al:: AJRCCM 1999, 159:1758.

3. Groner et al:: Nat Med 1999, 10:1209.

P147 Relationship between systemic arterial pressure (SAP) and $\mathrm{EtCO}_{2}$ in patients with hemorrhagic shock

Š Grmec*, Z Zabavnik†, M Batišta†, P Klemen* and Š Mally*

${ }^{\star}$ EMS-PHU Maribor; ${ }^{\dagger}$ Education Hospital Maribor; Ljubljanska 5, 2000 MB, Slovenia

Introduction: Capnometry may be useful in monitoring critical ED patients for early signs of shock or pulmonary embolus. However, $\mathrm{EtCO}_{2}$ levels must be interpreted in the context of other parameters giving information about the patient's clinical status. A fall in $\mathrm{EtCO}_{2}$ mav indicate decreased lung perfusion. If ventilation has not changed, the clinician should be alerted to reevaluate the patient for early signs of shock. The aim of this study was to examine the relationship between the changes in systemic arterial pressure (SAP) and in $\mathrm{EtCO}_{2}$ in hemodynamic status usually encountered during hemorrhagic shock.

Methods: Data were collected prospectively in EMS-PHU from February 1998 until August 1999. The study population was composed of adult (at least 18 years of age; mean age $47.8 \pm 13.4$; rang $18-76$ years) 68 patients ( 25 female, 43 male) with 3rd or 4th stage of hemorrhagic shock (trauma, Gl or respiratory hemorrhage). We measured SAP and $\mathrm{EtCO}_{2}$ for $1 \mathrm{~min}$ first $5 \mathrm{~min}$, and then each $5 \mathrm{~min}$ ). For each patient we determined MEES 1, MEES 2, APACHE II, $\triangle \mathrm{MEES}$. SAP and $\mathrm{EtCO}_{2}$ were measured with PROPAQ encore, Vital Sings Monitor, Protocol Systems, INC, Beaverton, OR, USA. We measured for each patient $\triangle \mathrm{EtCO} 2$ and $\triangle \mathrm{SAP}$ in interval from prehospital setting to hospitalization (674 simultaneous measurements were recorded). All $\mathrm{EtCO}_{2}$ and SAP values are reported in $\mathrm{mmHg}$.

Results: The mean and standard deviation of the $\triangle \mathrm{EtCO} 2$ was $12.52 \pm 6.36$ and of the $\Delta$ was $39.95 \pm 18.23$. Results are presented in the Figure. Statistics on the 68 individual
Figure

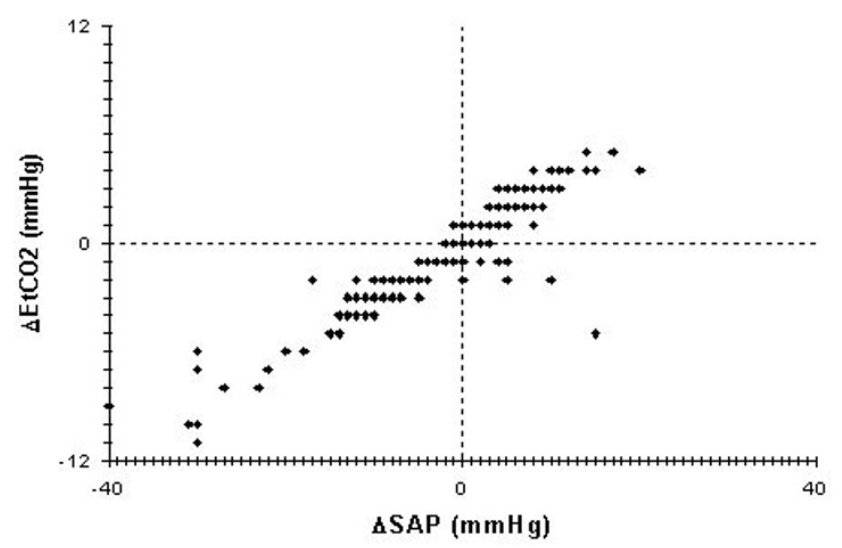

$\Delta \mathrm{SAP} 00.18+3.63 \times \Delta \mathrm{EtCO}_{2} ; \mathrm{r}=0.89 ; \mathrm{n}=674$.

correlations indicate $\triangle \mathrm{SAP}=0.06( \pm 0.18)+3.97( \pm 0.63) \times$ $\Delta \mathrm{EtCO} 2$ with $\mathrm{r}=0.91 \pm 0.08$.

Conclusion: A linear relationship does exist between the changes in SAP and in $\mathrm{EtCO}_{2}$ in patients with hemorrhagic shock. A $1 \mathrm{mmHg}$ change in $\mathrm{EtCO}_{2}$ is associated with $\sim 4$ $\mathrm{mmHg}$ change in SAP. It suggests that small changes in $\mathrm{EtCO}_{2}$ may be useful sign of significant changes in SAP (cardiac output). We conclude that the measurement of $\mathrm{EtCO}_{2}$ during hemorrhagic shock may be useful as a indicator of SAP and successful volume resuscitation.

\section{P148 Continuous monitoring of gastric carbon dioxide with optical fibers: European project COMOCADOF, in vitro evaluation}

A Lueger, D Scherr, G Brunner, W Trettnak*, A Mencaglia† and F Baldini ${ }^{\ddagger}$

Department of Int. Med., Karl-Franzens-University, Auenbruggerplatz 15, 8036 Graz, Austria; *Joanneum Research, 8010 Graz, Austria; ${ }^{+}$Prodotec SRL, 50127 Firenze, Italy; ${ }^{\ddagger} I R O E-C N R, 50127$ Firenze, Italy

Introduction: Gastric mucosal $\mathrm{PCO}_{2}$ is an important parameter to evaluate the splanchnic perfusion and to estimate the prognosis of critically ill patients. Saline and gas tonometry are the existing measurement methods, with air tonometry being the golden standard. The main disadvantage of this method is the relatively long equilibrium time, 
which only allows intermittent registrations and therefore cannot reflect rapid changes of $\mathrm{PrCO}_{2}$. The target of the COMOCADOF project was the development of an optical fiber sensor capable of a continuous monitoring of $\mathrm{PCO}_{2}$ inside the stomach, with high accuracy and long-term stability, able to track rapid $\mathrm{PrCO}_{2}$ changes.

Objectives: Evaluation of the feasibility of a measurement device using an optical fiber sensor for continuous measurement of gastric $\mathrm{PCO}_{2}$.

Methods: The COMOCADOF system consists of a fiberoptical catheter and the measurement unit. $\mathrm{A} \mathrm{CO}_{2}$ sensitive polymer layer is inserted into a probe head, which is fixed at the end of the optical fiber. The in vitro tests were performed in a glass test-tube containing $20 \mathrm{ml}$ of sample solution, which was maintained at a temperature of $37^{\circ} \mathrm{C}$. The sample solution (either physiological saline or a physiologically adjusted hydrochloric acid of $\mathrm{pH} 1.0$ ) was flushed with 6 gas mixtures containing 0.0 to $15.0 \%$ $\mathrm{CO}_{2}$ for defined time intervals and at a gas flow rate of 50 $\mathrm{ml} / \mathrm{min}$. The sampling rate was typically $0.8 \mathrm{~s}$. The tests were performed over a period of up to $48 \mathrm{~h}$. In addition, the influence of gastric juice, colored or turbid samples (such as e.g. enteral nutrition) on the stability of the optochemical sensor was tested.

Results: The table shows the original minimum requirements (target at the beginning of the project) and the

\section{Table}

\begin{tabular}{lcc}
\hline Parameters & Targets & Results \\
\hline Measurement range $(\mathrm{mmHg})$ & $15-120$ & $0-105^{\star}$ \\
Resolution at $0 \mathrm{mmHg}(\mathrm{mmHg})$ & 1 & 0.15 \\
Resolution at $120 \mathrm{mmHg}(\mathrm{mmHg})$ & 1 & 0.45 \\
Response time $\left(\mathrm{T}_{90}\right)(\mathrm{min})$ & 10 & $<2$ \\
Accuracy in range $40-105 \mathrm{mmHg}(\mathrm{mmHg})$ & \pm 2 & \pm 2 \\
Accuracy in range 0-40 mmHg $(\mathrm{mmHg})$ & \pm 2 & \pm 2 \\
Measurement period within accuracy $(\mathrm{h})$ & $24-72$ & 48 \\
\hline
\end{tabular}

${ }^{*}=\mathrm{PCO}_{2}>105 \mathrm{mmHg}$ not investigated, but possible.

achievements obtained up to now with the optical sensor for the detection of gastric carbon dioxide.

No significant influence of gastric juice, physiologically adjusted hydrochloric acid of $\mathrm{pH} 1.0$, colored or turbid samples (such as e.g. enteral nutrition) was seen.

Conclusion The COMOCADOF System allows direct continuous measurement of $\mathrm{CO}_{2}$ in gastric juice. The main targets of the project (response time, long-term stability and accuracy) were achieved. The system seems to perform better than the existing air tonometry method. Comparative studies with an air tonometry system both in vitro and in vivo are in progress.

\title{
P149 Assessment of a handy-type blood gas analyzer for measuring intramucosal pH (pHi)
}

\author{
H Dohgomori*, K Arikawa*, H Takamatsu* and Y Kanmura† \\ ${ }^{*}$ Division of Emergency Medicine, ${ }^{\dagger}$ Department of Anesthesiology and Critical Care Medicine, Kagoshima University Hospital, \\ Sakuragaoka, Kagoshima, 890-8520, Japan
}

Introduction: $\mathrm{pHi}$ is important in the evaluation of critically ill patients. When pHi data is needed quickly, a handy-type blood gas analyzer may be useful but it must be accurate. We therefore assessed the accuracy of a new handy-type blood gas analyzer (Opti, AVL, Switzerland; hereafter called $\mathrm{V}-\mathrm{O}$ ) in measuring $\mathrm{pHi}$ by comparing it with three other analyzers: (1) ABL520 (B-5), (2) ABL300 (B-3), (both from Radiometer, Holland), and (3) Compact 2 (AVL; V-C).

Methods: We infused normal saline into the balloon of a gastrointestinal tonometer (Instrumentarium Corp, Finland) located in the patient's stomach. After a 60 min equilibration time, the saline was withdrawn and the $\mathrm{CO}_{2}\left(\mathrm{PrCO}_{2}\right)$ measured by the four gas analyzers (the analysis of each sample of normal saline by the four analyzers were completed within $5 \mathrm{~min}$ ). At the same time, we withdrew and analyzed arterial blood. Then, $\mathrm{pHi}$ was calculated from the Henderson-Hasselbalch equation, and the $\mathrm{CO}_{2}$ gap $\left(\mathrm{PrCO}_{2}-\mathrm{PaCO}_{2}\right)$ was also calculated.

Results: Twenty-five $\mathrm{pHi}$ and $\mathrm{CO}_{2}$ gap values were obtained for each machine. The $\mathrm{CO}_{2}$ gaps for the various machines were as follows: B-5: $+4.5 \pm 2.6$ (mean $\pm \mathrm{SD}$ : $\mathrm{mmHg}$ ); B-3: $+1.5 \pm 3.1$; V-C: $+2.9 \pm 2.3$; and V-O: $-13.2 \pm 4.1$. The calculated $\mathrm{pHi}$ values were $7.336 \pm 0.056$ (mean $\pm S D$ ), $7.332 \pm 0.051 ; 7.330 \pm 0.051$, and $7.542 \pm 0.089$, respectively. With respect to the (B-5) results for $\mathrm{CO}_{2}$ gap, the values for correlation coefficient $(r)$, precision $(P)$ and bias (B) were as follows: (B-3) r, 0.900; $\mathrm{P}, 1.3 ; \mathrm{B},-3.1$; (V-C) r, 0.911 ; P, 1.4; B, -1.5; and (V-O) r, 0.790; P, 2.9; B, -18.3. A statistically significant relationship with $B-5$ was observed for each of these three groups, but the discrepancy between $V$ $\mathrm{O}$ and $\mathrm{B}-5$ was large.

Discussion: We can now use blood gas analyzers at the bedside and get results quickly. However, the value of $\mathrm{pHi}$ obtained differs according to the kind of analyzer used, a point that needs to be kept in mind. Our important finding is that the data obtained with the handy analyzer tested here differed quite considerably from those obtained using the other three. We conclude that although the new handy analyzer is convenient, it does not allow accurate calculations of $\mathrm{pHi}$ and $\mathrm{CO}_{2}$ gap. 


\section{P150 Gastric mucosal tonometry: should we adjust $\mathrm{PiCO}_{2}$ and $\mathrm{CO}_{2}$-gap to temperature?}

\section{H Vogelsang, T Uhlig, C Seifert and P Schmucker}

Department of Anaesthesiology, Medical University of Luebeck, Ratzeburger Allee 160, D-23538 Luebeck, Germany

Introduction: It has been under discussion for many years, whether acid-base-equilibrium during hypothermia should be managed using the alpha-stat or the $\mathrm{pH}$-stat concept. Several studies in cardiac patients undergoing hypothermic cardiopulmonary bypass (CPB) led to controversial results. Until now there are only few data available, on whether to adjust $\mathrm{PiCO}_{2}$ and $\mathrm{CO}_{2}$-gap to temperature or not. Thus we calculated $\mathrm{PiCO}_{2}$ and $\mathrm{CO}_{2}$-gap in cardiac surgery patients with and without temperature correction looking for differences in the prediction of postoperative complications.

Methods: After IRB approval, we studied 69 patients undergoing elective aortocoronary bypass surgery, ASA class II-III. In addition to standard monitoring each patient received a pulmonary artery catheter and an air-filled nasogastric tonometry catheter connected to a Tonocap ${ }^{\mathrm{TM}}$. Documentation of gastric-intramucosal $\mathrm{PCO}_{2}\left(\mathrm{PiCO}_{2}\right)$ followed at 15-min-intervals, documentation of hemodynamics, arterial and mixed-venous blood gas analyses and lactate followed seven times until extubation (T1-T7). We used mild hypothermic CPB and the alpha-stat concept. $\mathrm{PaCO}_{2}$ and $\mathrm{PiCO}_{2}$ were adjusted to temperature by the following algo- rithm: $\mathrm{PCO}_{2}{ }^{\mathrm{t}}=\mathrm{PCO}_{2} \times 100.0185 \times(\mathrm{t}-37)[\mathrm{mmHg}](\mathrm{t}=$ body temperature; $\mathrm{PCO}_{2}{ }^{\mathrm{t}}=$ temperature adjusted $\mathrm{PCO}_{2}$ ).

Statistical analyses were done using the paired t-test, $P<0.05$ was regarded as significant.

Results: There was a significant difference of about $3-4 \mathrm{mmHg}$ in temperature corrected $\mathrm{PiCO}_{2}$ and $\mathrm{PaCO}_{2}$ compared to uncorrected values. The $\mathrm{CO}_{2}$-gap ([Pi$\mathrm{Pa}] \mathrm{CO}_{2}$ ) differed significantly at T3, T6 and T7 in all patients and at T3 and T4 in patients with postoperative cardiopulmonary resuscitation $(n=5)$. However, the time course of measurements was equal.

Conclusion: For clinical applications, it is not necessary to adjust $\mathrm{PiCO}_{2}$ and $\mathrm{CO}_{2}$-gap to body temperature during mild hypothermic CPB with alpha-stat concept since no further information could be gained from the correction. In laboratory settings or deep hypothermic $\mathrm{CPB}$ with $\mathrm{pH}$-stat concept temperature adjustment of tonometric parameters may be helpful. In addition, the correction may also be useful in hyperthermia (septic patients). However, this has to be investigated in further studies.

\section{P151 Evaluation of $\mathrm{PCO}_{2}$ measurements using a gastric air tonometer located in non-mixing solution}

Y Sato, H Inoue, K Ikegami, T Seki, F Morimoto, Y Nagai*, T Sekiguchi* and M Nakamura*

Trauma and Critical Care Center, Koshigaya Hospital, Dokkyo University School of Medicine, 2-1-50 Minami-Koshigaya, Koshigaya, Saitama, Japan; ${ }^{\star} R$ and D Center, Nihon Kohden Corp., Tokyo, Japan

Introduction: Many studies reported that measurements of gastric $\mathrm{PCO}_{2}$ with the air tonometer method were more precise than those obtained with saline tonometer. Gastric tonometers, however, were usually validated in vitro in a solution bubbled with $\mathrm{CO}_{2}$. In this study, we evaluated the accuracy and equilibration characteristics of $\mathrm{PCO}_{2}$ in the balloon of a gastric air tonometer located in saline solution either with or without bubbling of $\mathrm{CO}_{2}$.

Methods: A gastric tonometer was immersed in a $0.9 \%$ saline solution maintained at $37^{\circ} \mathrm{C}$, in which certified calibration gases at three different $\mathrm{CO}_{2}$ concentrations $(5 \%$, $9 \%$, and $12 \%$ ) were bubbled. When solution was saturated with $\mathrm{CO}_{2}$ gas, $\mathrm{PCO}_{2}$ in the balloon was measured every $10 \mathrm{~min}$ for $30 \mathrm{~min}$ by an infra-red analyzer with or without $\mathrm{CO}_{2}$ bubbling. Bias and precision were calculated from the measured and expected $\mathrm{PCO}_{2}$ values.

Results: Under $\mathrm{CO}_{2}$ bubbling, relatively small bias $(-4 \% \sim 9 \%)$ was seen at all three $\mathrm{CO}_{2}$ concentrations. On the contrary, large negative bias (26\%-39\%) was observed without bubbling.

Conclusion: It has been demonstrated that quick equilibration of $\mathrm{PCO}_{2}$ between inside and outside of the tonometer balloon needs the mixing of sample solution. This result strongly suggests that measurements of $\mathrm{PCO}_{2}$ with the air tonometer would underestimate gastric $\mathrm{PCO}_{2}$ when it is located in the gastric juice with little mixing.

\section{P152 Influence of prone position on gastric mucosal-arterial $\mathrm{PCO}_{2}$ gradient}

\section{P Kiefer, A Morin, H Wiedeck, M Georgieff and P Radermacher \\ Universitätsklinik f. Anästhesiologie, Universität, D-89070 Ulm, Germany}

Introduction: In patients with acute lung injury (ALI), the prone position is a well-established method to improve gas exchange by reopening atelectasis and secret drainage. This approach might lead, however, to increased intra-abdominal pressure and thereby impaired gastric mucosal perfusion. Therefore, we studied the effect of the 
prone position on both intragastric pressure and gastric mucosal-arterial $\mathrm{PCO}_{2}$ gradients in patients with ALI.

Patients/methods: So far, 10 patients with ALI $\left(\mathrm{PaO}_{2} / \mathrm{FiO}_{2}<250 \mathrm{mmHg}\right)$ and clinical indication for mechanical ventilation in the prone position were studied. In addition to ventilator settings, systemic hemodynamics and gas exchange, we semi-continuously measured the gastric mucosal $\mathrm{PCO}_{2}\left(\mathrm{PCO}_{2} \mathrm{gm}\right)$ (Tonocap) via a nasogastric tube as well as the intragastric pressure continuously via one lumen of the nasogastric tube using a common pressure transducer. After $60 \mathrm{~min}$ of stable conditions baseline measurements were made. Then the patient was turned to prone position and further measurements were obtained 60 and 120 min later.

Results/statistics: See Table. Friedmann test/StudentNewman-Keuls: ${ }^{\#} P<0.05$ vs baseline.

Conclusion: Despite major individual variability, we could not find a significant overall change in gastric mucosal-arterial $\mathrm{PCO}_{2}$ gradient after turning to prone
Table

\begin{tabular}{lccc}
\hline (median/percentile) & Baseline & $60 \mathrm{~min}$ & $120 \mathrm{~min}$ \\
\hline $\mathrm{Ci} / / \mathrm{min} / \mathrm{m}^{2}$ & $3.8 / 4.7-3$ & $3.8 / 4.1-3.3$ & $3.9 / 4.6-3.4$ \\
$\mathrm{MAP} \mathrm{mmHg}$ & $85 / 93-77$ & $81 / 88-66$ & $78 / 88-70$ \\
$\mathrm{CVP} \mathrm{mmHg}$ & $14 / 15-12$ & $14 / 15-8$ & $13 / 16-10$ \\
$\mathrm{PCO}_{2} \mathrm{gm} \mathrm{mmHg}$ & $53 / 57-49$ & $57 / 63-55$ & $58 / 71-51$ \\
$\mathrm{PCO}_{2}$ art mmHg & $47 / 50-42$ & $48 / 52-44$ & $44 / 48-43$ \\
Gastric pressure mmHg & $12 / 14-6$ & $12 / 16-9$ & $10 / 13-7$ \\
$\mathrm{PCO}_{2} \mathrm{gm}-\mathrm{PCO}_{2}$ art mmHg & $6 / 11-3$ & $8 / 17-6$ & $10 / 18-3$ \\
\hline
\end{tabular}

position. Increased intragastric pressure $(>5 \mathrm{mmHg}$ ), however, was associated with an increased gastric mucosal-arterial $\mathrm{PCO}_{2}$ gradient despite stable systemic hemodynamics. Positioning induced variations of intraabdominal pressure, hence, may impair gastric mucosal energy balance possibly due to compromised regional blood flow.

\section{P153 Systemic and splanchnic hemodynamics, metabolism and $\mathrm{PCO}_{2}$-gap during septic shock induced by live $E$. coli infusion in dogs}

C Lagoa, LFP Figueiredo, R Cruz Jr, E Silva and M Rocha e Silva

Heart Institute (InCor), University of São Paulo Medical School, Brazil. Av. Dr. Enéas de Carvalho Aguiar, 44 CEP $05403-000$

São Paulo-SP, Brazil

Background: The effects of intravenous live bacteria infusion on the time course of splanchnic oxygenation variables have not been adequately defined, as well as the effects of large volume crystalloid infusion on these variables.

Methods: Twenty-seven anesthetized mongrel dogs $(17.1 \pm 1.77 \mathrm{~kg})$ were challenged by a $15 \mathrm{~min}$ intravenous infusion of live $E$. coli $\left(6 \times 10^{9} \mathrm{CFU} / \mathrm{kg}\right)$ and followed for 90 $\min$. The animals were then randomized in two groups over 60 min: CT (controls, $n=13$ ), no fluid infusion or LR (lactated Ringer's $32 \mathrm{ml} / \mathrm{kg} / \mathrm{h}, \mathrm{n}=14)$. Cardiac index $(\mathrm{Cl}$ in $\mathrm{l} / \mathrm{min} / \mathrm{m}^{2}$ ), mean arterial pressure (MAP in $\mathrm{mmHg}$ ), mesenteric blood flow (MBF in $\mathrm{ml} / \mathrm{min}$, ultrasonic flowprobe), oxygen-derived variables, lactate levels (in $\mathrm{mmol} / \mathrm{l}$ ) and gastric $\mathrm{PCO}_{2}$ (gastric tonometry in $\mathrm{mmHg}$ ) were assessed throughout the 165 min experimental protocol. $\mathrm{PCO}_{2}$-gap was defined as the difference between gastric and arterial $\mathrm{PCO}_{2}$ and lactate flux was defined by the standard formula: ([portal lactate levels-arterial lactate levels] $\times \mathrm{MBF}$ ).

Results: Data are presented as mean $\pm S E$. E.coli infusion significantly reduced MAP, $\mathrm{Cl}, \mathrm{MBF}$, and increased $\mathrm{PCO}_{2}$ gap in both groups. Fluid infusion increased $\mathrm{Cl}$, systemic $\mathrm{DO}_{2}$ and stabilized $\mathrm{PCO}_{2}$-gap. Although MBF was similar in both groups, only controls presented gut lactate pro-

Table 1

Time course of MAP, Cl, MBF, Lactate Flux and $\mathrm{PCO}_{2}$-gap at baseline, 15, 105 and 165 min.

\begin{tabular}{lcccccccc}
\hline Time & \multicolumn{2}{c}{ Baseline } & \multicolumn{2}{c}{$15 \mathrm{~min}$} & \multicolumn{2}{c}{$105 \mathrm{~min}$} & \multicolumn{2}{c}{$165 \mathrm{~min}$} \\
\hline Groups & $\mathrm{CT}$ & $\mathrm{LR}$ & $\mathrm{CT}$ & LR & $\mathrm{CT}$ & LR & CT & LR \\
MAP & $109 \pm 3.8$ & $104 \pm 3.7$ & $97 \pm 4.7$ & $99.7 \pm 4.3$ & $73.9 \pm 5.7$ & $68.7 \pm 4.6$ & $88.3 \pm 5.0$ & $93.1 \pm 3.9$ \\
$\mathrm{Cl}$ & $2.8 \pm 0.2$ & $2.89 \pm 0.2$ & $1.97 \pm 0.1$ & $2.14 \pm 0.2$ & $2.06 \pm 0.2$ & $1.99 \pm 0.2$ & $2.04 \pm 0.2$ & $2.93 \pm 0.2$ \\
$\mathrm{MBF}$ & $408 \pm 44$ & $410 \pm 51$ & $208 \pm 19$ & $292 \pm 42$ & $279 \pm 46$ & $194 \pm 25$ & $335 \pm 53$ & $304 \pm 36$ \\
Lact. flux & $-41.8 \pm 44$ & $8.48 \pm 35$ & $8.8 \pm 18$ & $16.1 \pm 26$ & $-27.1 \pm 48$ & $-30.6 \pm 39$ & $50.9 \pm 63$ & $-58.4 \pm 60$ \\
$\mathrm{PCO}_{2}$-gap & $15 \pm 3.79$ & $9.69 \pm 1.62$ & $18.8 \pm 3.41$ & $12 \pm 2.09$ & $43.9 \pm 4.84$ & $34.2 \pm 2.6$ & $52.5 \pm 4.7$ & $34.8 \pm 3.67$ \\
\hline
\end{tabular}


duction. Table 1 shows the time course of MAP, Cl, MBF, lactate flux and $\mathrm{PCO}_{2}$-gap of the two groups.

Conclusion: Although large-volume crystalloid infusion fails to restore mesenteric and gastric mucosal perfusion, it seems to prevent gut lactate production in a canine model of experimental septic shock.

\section{P154 Routine blood gas analysis does not provide information on regional metabolism of the stomach obtained by gastric tonometry in patients with left ventricular failure}

J Graf, E Karassimos and $\mathbf{U}$ Janssens

Medical Clinic I, RWTH Aachen, Pauwelsstr. 30, 52057 Aachen, Germany

Purpose of the study: To define the association between variables reflecting regional perfusion and metabolism of the stomach obtained by gastric air tonometry with routine blood gas analysis and global hemodynamics in patients with cardiac failure.

Methods: In 50 mechanically-ventilated patients with severe left ventricular failure gastric intramucosal $\mathrm{PCO}_{2}$ $\left(\mathrm{PgCO}_{2}\right)$ was assessed utilising the Tonocap. Simultaneously hemodynamics were measured and routine blood gas analysis was carried out. Independent variables were correlated and the discriminative power to predict a low $\mathrm{pHi}<7.32$ was calculated using Receiver Operating Characteristics (ROC).

Results: 564 paired measurements of cardiorespiratory and gastric tonometry variables were collected. Neither global hemodynamics nor arterial lactate values correlated with one of the tonometric parameters or patients' outcome at any timepoint. Correlation of arterial $\mathrm{PCO}_{2}$, arterial $\mathrm{pH}$, arterial bicarbonate, and base excess with $\mathrm{PgCO}_{2}$ was only weak. According to the areas under $\mathrm{ROC}$ prediction of $\mathrm{pHi}<7.32$ was excellent for $\mathrm{PgCO}_{2}$ and $\mathrm{CO}_{2}$ Gap (difference of $\mathrm{PgCO}_{2}$ and arterial $\mathrm{PCO}_{2}$ ) (areas under ROC curve $0.90 \pm 0.01$ and $0.92 \pm 0.01$, cutoff values $51 \mathrm{mmHg}$ and $13 \mathrm{mmHg}$, respectively).

Conclusion: Both $\mathrm{PgCO}_{2}$ and $\mathrm{CO}_{2}$ Gap are reliable predictors of low $\mathrm{pHi}$, thought to reflect gastric mucosal hypoperfusion. None of the cardiorespiratory parameters were capable of predicting a $\mathrm{pHi}<7.32$. Thus, in patients with severe left ventricular failure assessment of regional perfusion indices using gastric tonometry cannot be replaced by one of the tested routinely applied monitoring modalities.

\section{P155 Gastric-arterial $\mathrm{pCO}_{2}$ gradient, but not lactate levels, is related to multiple organ dysfunction assessed by SOFA score in septic patients}

E Silva, M Kai, M Assumpção, PC Martins, AG Garrido, $\mathbf{S}$ Blecher and E Knobel

Albert Einstein Hospital, Av. Albert Einstein, 627 5th Floor, São Paulo, 05651-90, Brazil

Introduction: The gut hypoperfusion could contribute to development of multiple organ dysfunction in septic patients. However, there is no definitive study correlating the time course of gastric mucosal $\mathrm{PCO}_{2}$ and organ dysfunction.

Methods: We have studied prospectively 27 adult severe septic patients consecutively admitted in two large mixed ICUs. Each patient had a nasogastric tonometer and a pulmonary artery catheter. Every $8 \mathrm{~h}$, systemic hemodynamic and oxygen variables, plasma lactate levels and gastric mucosal $\mathrm{pCO}_{2}\left(\mathrm{PgCO}_{2}\right)$ were measured. All these variables were measured on inclusion day (Day 0 ) and on the 1 st, $2 \mathrm{nd}$ and 3 rd days. $\mathrm{pCO}_{2}$-gap was calculated as the difference between $\mathrm{PgCO}_{2}$ and $\mathrm{PaCO}_{2}$. Daily, we measured the SOFA score to characterize organ dysfunction evolution. We used the median $\mathrm{pCO}_{2}$-gap and lactate values of each day and time course of these variables to correlate to organ dysfunction and outcome (mortality on day 28). Changes over time were analyzed using a Kruskal-Wallis test and the relative risk (RR) was calculated.
Results: The median age was 55 years and median APACHE II score was 18.5 . The overall mortality rate was $52 \%$. Table 1 shows the RR and $\mathrm{Cl} 95 \%$ of each variables on day $0,1,2$ and 3 . The best cutoff values of $\mathrm{pCO}_{2}$-gap, lactate values and SOFA score were $15 \mathrm{mmHg}, 2 \mathrm{mEq} / \mathrm{l}$ and 11 , respectively. On the 0,1 st, 2 nd and 3rd days, the patients that had $\mathrm{pCO}_{2}$-gap values of more than $14 \mathrm{mmHg}$, either the SOFA score remained high or the

\section{Table 1}

RR and $\mathrm{Cl} 95 \%$ of each variable to estimate outcome on 28th

day

\begin{tabular}{lcccc}
\hline & Day 0 & Day 1 & Day 2 & Day 3 \\
\hline pCO $_{2}$-gap & 2.1 & 2.0 & 2.2 & 2.0 \\
& $(1.1-3.7)$ & $(1.0-3.7)$ & $(1.1-4.3)$ & $(1.0-3.7)$ \\
SOFA & 1.4 & 2.2 & 2.1 & 2.3 \\
& $(0.7-2.8)$ & $(1.1-4.3)$ & $(1.1-3.7)$ & $(1.2-4.2)$ \\
Lactate & 0.8 & 2.3 & 1.9 & 1.6 \\
& $(0.3-1.6)$ & $(1.0-5.4)$ & $(0.8-4.3)$ & $(0.7-3.5)$ \\
\hline
\end{tabular}


patients died on the 10th day $(P<0.05)$. In contrast, on the same days, lactate levels did not discriminate either organ dysfunction development or death on the 10th day.
Conclusions: There was a good correlation between $\mathrm{pCO}_{2}-$ gap and multiple organ dysfunction assessed by SOFA score. Lactate levels were not able to predict outcome.

\title{
P156 The relationship between data of gastric tonometry by determination of polymorphonuclear leukocytes (PMNs, i.e., myeloperoxidase activity) and gut mucosal hypoperfusion in elective cardiac surgical patients
}

\author{
A Pietsch, T Uhlig, H Vogelsang and P Schmucker \\ Department of Anesthesiology, Medical University of Luebeck, Ratzeburger Allee 160, 23538 Luebeck, Germany
}

Introduction: The expression of polymorphonuclear leukocytes (PMNs) as a result of local and systemic inflammation in patients undergoing cardiac surgery by using the CPB is considered to be an important cofactor in tissue hypoxia and the pathogenesis of multiple organ failure [1]. Therefore, an early detection of tissue hypoxia is very useful for the prevention of MOFS. It has been shown that gastric tonometry improves clinical applicability of gut mucosal oxygenation monitoring [2]. In a previous paper the correlation between gastric tonometry and PMNs was warranted [3]. This was done in the following study.

Methods: Following institutional approval, 66 adult male patients undergoing elective cardiac surgery using cardiopulmonary bypass were studied. They were monitored with the Tonocap ${ }^{\mathrm{TM}}$ in addition to standard monitoring (ECG, CVP, arterial pressure) and the pulmonary artery catheter. The $\mathrm{PiCO}_{2}$ was measured every 15 min after admission to the ICU. In addition, documentation of $\mathrm{CO}_{2}$ gap, arterial and pulmonary arterial hemodynamics, arterial and mixed-venous blood gas analyses and lactate followed four times until extubation. The patients were shifted into three groups by using the terciles of preopera- tive expression of the PMNs (group I $<1451 \mu \mathrm{g} / \mathrm{l}$; group II $\leq 2487 \mu \mathrm{g} / \mathrm{l}$; group III >2487 $\mu \mathrm{g} / \mathrm{l})$.

Results: The course of $\mathrm{CO}_{2}$-gap was parallel to the PMNs. Both parameters increased after admission to the ICU. Using the Wilcoxon-Wilcox test the increase in $\mathrm{CO}_{2}$ gap and PMNs was significant in all groups. These differences did not occur in the parameters obtained from blood gas analyses (BE, lactate, mixed venous saturation) and in hemodynamic parameters.

Conclusions: Gastric tonometry is superior to other techniques in detecting dysfunctions of gut oxygenation if $\mathrm{PMNs}$ are used as a biochemical indicator. Questions of therapeutic interventions in increasing $\mathrm{CO}_{2}$-gaps and PMNs need further investigations.

\section{P157 Detection of perfusion failure during open heart surgery with sublingual $\mathrm{PCO}_{2}$}

\author{
H Inoue, I Sato, N Hitosugi, Y Takano, M Miyake, Y Sato, K Ikegami, Y Nagai ${ }^{\star}$, T Sekiguchi and M Nakamura* \\ Koshigaya Hospital Dokkyo University School of Medicine, 2-1-50, Minami-Koshigaya, Koshigaya, Saitama, Japan; *Nihon \\ Kohden Corp, Tokyo, Japan
}

Background: Gastric tonometry has been used to detect intramucosal acidosis. However, there remains controversies about the methodology and interpretation of the results. In recent years, sublingual $\mathrm{PCO}_{2}\left(\mathrm{PSLCO}_{2}\right)$ has been introduced as a noninvasive method to detect perfusion failure. Yet, there has not been enough evidence to prove that the $\mathrm{PSLCO}_{2}$ is clinically of use.

Purposes: To define the efficacy of $\mathrm{PSLCO}_{2}$.

Methods: We investigated 6 patients who underwent open-heart surgery. $\mathrm{PSLCO}_{2}$ were recorded continuously with a $\mathrm{PCO}_{2}$ sensor based on an ion-sensitive field effect transistor (ISFET, NIHON KOHDEN). Arterial lactate was also measured.
Result: A substantial increase of $\mathrm{PSLCO}_{2}$ came into our notice from $377 \mathrm{mmHg}$, at the time of induction to 5712 $\mathrm{mmHg}$, during the cardiopulmonary bypass (CPB). Gradual decrease of $\mathrm{PSLCO}_{2}$ after the aorta declamping was observed down to $485 \mathrm{mmHg}, 6 \mathrm{~h}$ after the operation. Significant increase in $\mathrm{PSLCO}_{2}$ indicates that oxidization is not fairly sustained to the tissue during CPB despite enough pump flow. The increase in blood lactate concentration was followed parallel to $\mathrm{PSLCO}_{2}$ while CPB was performed.

Conclusion: $\mathrm{PSLCO}_{2}$ is an attractive parameter to be served for the swift detection of perfusion failure during open-heart surgery. 


\section{P158 Is muscular mass affected by L-Carnitine levels in critically ill patients?}

M Moukas, P Dimou, A Chalazonitis, A Patsalides, G Tsimitselis, S Georgakopoulou, N Koudounis, M Vasiliou and P Behrakis Intensive Care Unit and Department of Radiology, Hellenic Red Cross Hospital, 1 Erythrou Stavrou str. Athens 11526, Greece

Introduction: The aim of our study is to investigate the change of blood and muscle Carnitine levels in patients of the Intensive Care Unit and investigate how the change in Carnitine levels affects muscular mass.

Method: In our double blind clinical study, forty-six ICU patients were randomly divided into two groups. Patients with either renal or liver insufficiency were excluded. Both groups of patients were on enteral or parenteral nutrition with $30 \mathrm{kcal} / \mathrm{kg} /$ day intake with $1 \mathrm{~g} / \mathrm{kg} /$ day protein intake. The mean duration of hospitalization was $23.6 \pm 8$ days for group $A$ and $26.9 \pm 9$ days for group B. All patients were under physical therapy for $60 \mathrm{~min} /$ day. Only group B patients received intravenous supplements containing $100 \mathrm{mg} / \mathrm{kg} /$ day L-Carnitine. Total and free Carnitine were measured from the blood of each patient at the time of admission and at the time of exit from the ICU. Muscle Carnitine levels were also studied with a triceps muscle biopsy at the same time. Muscle mass thickness was estimated by the same utrasonographer in all cases. A 10 $\mathrm{MHz}$ frequency, linear high-resolution transducer was held longitudinal to the biceps muscle in the plane of maximum thickness.

Results: All values are expressed as mean $\pm S D$ on admission and exit from the ICU. Blood results for Group A patients are: total Carnitine $(\mathrm{nmol} / \mathrm{ml}) 78.14 \pm 23.17$ and
$50.56 \pm 21.23(P<0.001)$ and free Carnitine $(\mathrm{nmol} / \mathrm{ml})$ was $69.41 \pm 23.77$ and $42.34 \pm 18.00(P<0.001)$ respectively. Group $B$ has the following results: total Carnitine $(\mathrm{nmol} / \mathrm{ml}) 74.08 \pm 24.51$ and $529.92 \pm 228.45(P<0.001)$ and free Carnitine $(\mathrm{nmol} / \mathrm{ml}) \quad 65.59 \pm 25.07$ and 459.43 $\pm 162.12(P<0.001)$.

Muscle biopsy results are as follows: Group A patients had free Carnitine $1.50 \pm 0.33$ on admission and $1.06 \pm 0.19$ on exit $(P<0.0001)$, and total Carnitine $1.70 \pm 0.29$ and $1.10 \pm 0.21$ respectively $(P<0.0001)$. In Group B patients, free Carnitine was $1.53 \pm 0.31$ on admission and $1.99 \pm 0.15$ on exit $(P<0.0001)$, and total Carnitine $1.67 \pm 0.29$ and $2.23 \pm 0.16$ respectively.

Muscle mass $(\mathrm{cm})$ measurement results for group $A$ patients are: $2.6 \pm 0.5$ and $2.3 \pm 0.6(P<0.01)$ whereas for group $B$ patients they are $2.09 \pm 0.5$ and $2.31 \pm 0.1$ $(P<0.001)$ respectively.

Conclusions: In group A patients, blood and muscle Carnitine are decreased. Muscle mass is concomitantly decreased. The opposite results are obtained from group B (L-Carnitine supplementation). We conclude that L-Carnitine supplementation prevents muscle atrophy in critically ill ICU patients.

\section{P159 Pseudocholinesterase and albumin in infants with cirrhosis}

\section{LJ Popoviê, A Antabak, B Æupanëiê and V Vegar}

Surgical Clinic and Department of Anaesthesiology, Clinical Hospital Centre Rebro, Kispaticeva 12, 10000 Zagreb, Croatia

Objectives: Pseudocholinesterase (PChE) is an enzyme (EC 3.1.1.8) and like albumin is synthesised in the liver. Although the two proteins are synthesised in the liver they are not interdependent and both have been used independently as an index of liver function. Because of the contrary literature data, the aim of the present study was to measure PChE activity and albumin concentration in healthy infants and compare them with adult values $[1,2]$. We also compared PChE and albumin values between healthy infants and infants with biliary atresia and cirrhosis. PChE activity and albumin concentration were measured in 25 infants with biliary atresia and accompanying liver cirrhosis before Kasai operation, in 10 healthy infants, as well as in 10 healthy adults undergoing elective surgery.

Methods: PChE activity was determined by the spectrophotometric method of Ellman, using butyrylthiocholine as substrate. Plasma albumin concentration was measured by electrophoresis.

Results: In infants with liver cirrhosis, PChE values were about $50 \%$ lower then in healthy infants of the same age i.e. 2-6 months $(P<0.001)$. In healthy infants PChE activity was lower by $20 \%$ than in adults $(P<0.001)$. Significantly lower albumin concentration in all infants than in adults has also been established $(P<0.05)$.

Conclusion: Our results showed diminished synthesis of PChE and albumin in liver cells of infants (2-6 months). These observations would suggest that infants might be expected to differ significantly from adults in the extent to which drugs might be protein bound and in the rates of hydrolysis of ester-type drugs. It is well known that PChE is involved in the hydrolysis of muscle relaxants succinyl- 
choline and mivacurium. According to our results, 50\% lower PChE activity in infants with biliary cirrhosis may cause prolonged duration of mentioned drugs.
References:

1. Ecobichon DJ, Stephens DS: Perinatal development of human blood esterase. Clin Pharmacol Ther 1973, 14:41-47.

2. Strauss AA, Mondanlou HD: Transient plasma cholinesterase deficiency in preterm infants. Dev Pharmcol Ther 1986, 9:82-87.

\section{P160 Serum albumin and mortality risk in critically ill patients}

\section{FHY Yap, GM Joynt and TA Buckley}

Department of Anaesthesia and Intensive Care, Prince of Wales Hospital, Shatin, HKSAR, China

Introduction: It had been shown that serum albumin measured within $48 \mathrm{~h}$ of ICU admission is as accurate as the APACHE II score in predicting hospital mortality. If this result could be confirmed, then albumin would be a cheaper and more convenient predictor of mortality than APACHE II.

Aim: To evaluate serum albumin as a predictor of hospital mortality in critically ill patients.

Methods: All consecutive adult ICU admissions over an 18 month period (April 1997 to September 1998) were reviewed. The data were retrieved from a computerised database. Serum albumin concentrations between survivors and nonsurvivors were compared. Accuracy of outcome prediction using albumin was assessed by area under the curve (AUC) of the receiver operator characteristics (ROC) curve.

Results: 1003 records were reviewed. There were 647 $(64.5 \%)$ survivors and $356(35.5 \%)$ nonsurvivors. The sur- vivors had a significantly higher serum albumin both on ICU admission and in the first $72 \mathrm{~h}$ after admission $(P<0.001)$ (see Fig. 1). In both groups, the serum albumin fell markedly after ICU admission. The ROC curves for hospital mortality are shown in Fig. 2. The AUC of APACHE II (0.79) is significantly higher than that of admission albumin (0.63) and albumin at $24 \mathrm{~h}(0.64)(P<0.001)$. We used regression analysis to combine APACHE II with admission albumin (AUC 0.80) and APACHE II with albumin at $24 \mathrm{~h}$ (AUC 0.79 ) to predict hospital mortality. The addition of albumin did not significantly improve the accuracy of outcome prediction over that of APACHE II alone.

Conclusion: There is an association between serum albumin concentration and hospital mortality. However, as a single variable, serum albumin is a poor predictor of hospital outcome. Combining APACHE II with admission albumin or albumin at $24 \mathrm{~h}$ did not improve the accuracy of outcome prediction over that of APACHE II score alone.
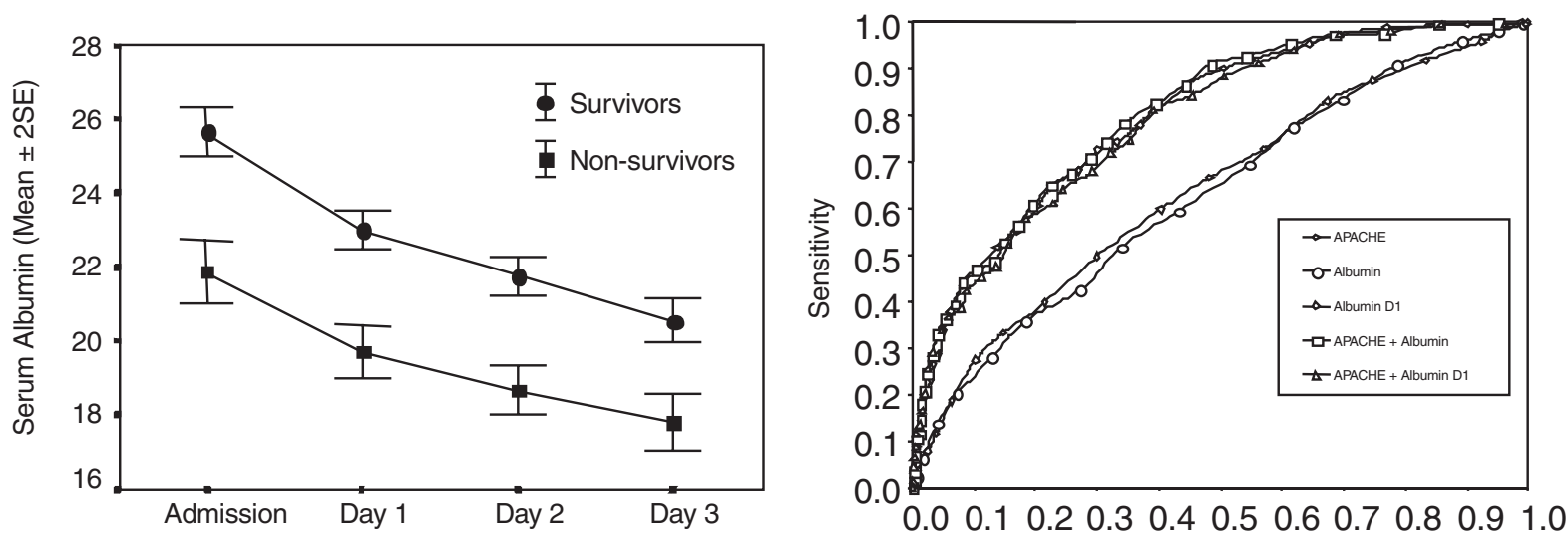


\section{P161 Parenteral nutrition (PN) depresses hepatic albumin synthesis in septic rats}

\section{MJ O’Leary ${ }^{*}$, M Koll ${ }^{\dagger}$, CN Ferguson $^{*}$, CJ Hinds*, VR Preedy ${ }^{\dagger}$, JH Coakley* and PJ Garlick ${ }^{\ddagger}$}

${ }^{*}$ Department of Intensive Care, St. Bartholomew's Hospital, London EC1A 7BE, UK; ${ }^{+}$Department of Nutrition and Dietetics, King's College London, London SE1 8WA, UK; 'Department of Surgery, SUNY, Stony Brook, New York, USA

Serum albumin concentration is frequently used as an indicator of nutritional response in patients receiving PN. The acute-phase response following injury and sepsis is characterised by increased hepatic synthesis of specific secreted proteins while production of albumin is decreased. We have investigated the effect of acute administration of $\mathrm{PN}$ on absolute and relative rates of hepatic albumin synthesis during sepsis in vivo. Two groups of male Wistar rats (mean weight $239 \mathrm{~g}$ ) underwent caecal ligation and puncture (CLP), with a third group as unoperated controls allowed free access to chow and water (ad lib). Between 18 and $24 \mathrm{~h}$ later CLP survivors were infused by tail vein with $0.9 \% \mathrm{NaCl}$ or a $\mathrm{PN}$ solution delivering $33 \%$ of calculated daily energy and protein requirements. Total hepatic protein synthesis rate (TPS), albumin synthesis rate (TAS) and relative albumin synthesis rate (RAS) were determined with a flooding dose of ${ }^{3} \mathrm{H}$-phenylalanine and the use of anti-rat albumin antibody to isolate the protein for radioactive counting.

Results: (mean \pm SEM) are displayed in the table, with statistical analysis by ANOVA.
Table

\begin{tabular}{lccc}
\hline Group (n) & ad lib (5) & $0.9 \% \mathrm{NaCl}(8)$ & $\mathrm{PN}(9)$ \\
\hline TPS (mg/day) & $1395.6 \pm 34.7$ & $1811.9 \pm 91.0^{+}$ & $1948.9 \pm 138.7^{+}$ \\
TAS (mg/day) & $167.9 \pm 6.6$ & $102.1 \pm 18.7^{\star}$ & $73.8 \pm 6.1^{+\#}$ \\
RAS (\%) & $11.77 \pm 0.42$ & $5.59 \pm 0.9^{+}$ & $3.52 \pm 0.34^{+\bullet}$
\end{tabular}

$+P=<0.00001,{ }^{*} P=0.0011$ compared with ad lib; $\#=0.1, \cdot P=0.0411$ compared with $0.9 \% \mathrm{NaCl}$.

We have demonstrated that whereas TPS is increased in sepsis, TAS falls. Provision of PN has no effect on TPS, but significantly further depresses RAS. Reduced hepatic albumin synthesis in sepsis is not reversed by substrate provision, hence changes in serum albumin concentration are unlikely to be a useful monitor of efficacy of PN.

Acknowledgement: MJ O'Leary was supported by The British Journal of Anaesthesia, BMI/Columbia Healthcare Ltd., and The Joint Research Board of St. Bartholomew's Hospital.

\section{P162 Measurement of gastric emptying in ICU patients with ${ }^{13} \mathrm{C}$-acetate}

\section{Ch Goeters, C Schulzki, A Wenn, U Hölscher* and H Van Aken}

Department of Anaesthesiology and Intensive Care Medicine, University of Münster, Albert-Schweitzer-Str. 33, D-48149

Münster; *Faculty of Applied Physics and Engineering, University of Applied Sciences, Münster, Germany

Introduction: In clinical practice, gastric emptying and the success of gastric feeding is assessed by measurement of gastric residuals. A new setting easily allows bedside measurements in ventilated ICU-patients by ${ }^{13} \mathrm{C}$-technology to evaluate gastric transport.

Methods: ${ }^{13} \mathrm{C}$-acetate added to tube feeding will be absorbed into the blood stream from the duodenum and oxidised to ${ }^{13} \mathrm{CO}_{2}$ in the liver. The ratio of ${ }^{13} \mathrm{CO}_{2} /{ }^{12} \mathrm{CO}_{2}$ in the expired air can be determined by infrared isotopeselective spectrometry (IRIS). Increasing expiratory concentrations of ${ }^{13} \mathrm{CO}_{2}$ indicate transport of the applied tracer from the stomach to the duodenum. In 11 healthy volunteers breathing spontaneously via a tight CPAP mask on a Dräger Evita respirator system, ${ }^{13} \mathrm{CO}_{2}$ enrichment in the expired air was measured for two hours after ingestion of $150 \mathrm{mg}{ }^{13} \mathrm{C}$-acetate in $50 \mathrm{ml}$ tube feeding. In nine ventilated ICU patients measurements were performed to determine expiratory ${ }^{13} \mathrm{CO}_{2} /{ }^{12} \mathrm{CO}_{2}$ ratios after administration of the same amount of tracer and tube feeding via gastric tube. Gastric reflux and the total amount of tube feeding were recorded before measuring.

\section{Figure}

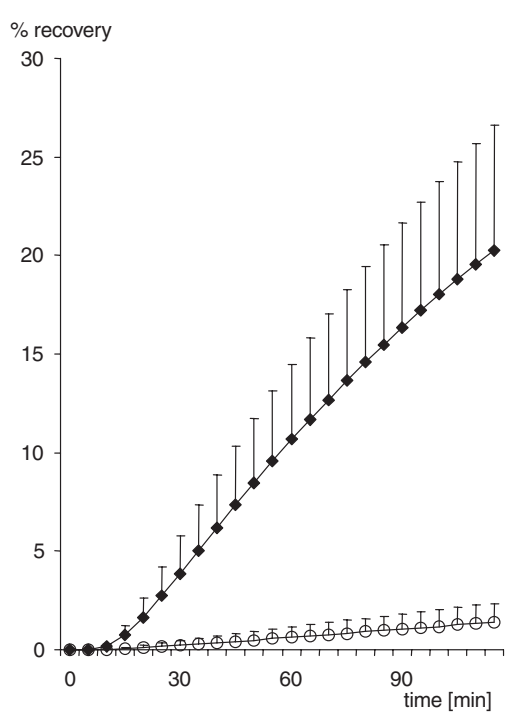

Cumulative recovery (mean \pm SD) in volunteers $(\downarrow)$ and ICU-patients (O) 
Results: The new setting was validated in volunteers. About 20 to 40 min after ingestion of the tracer, peak values (\% of ${ }^{13} \mathrm{C}$ dose/h) were recorded. Within two hours the cumulative recovery was $20 \%$. In the ICU patients only a small quantity of the applied tracer $(<5 \%)$ was recovered independent of reflux amounts.
Conclusion: We could not quantify gastric emptying by this new setting in ICU patients. Failure might be due to changes in transport mechanisms, resorption in the duodenum, metabolism and pool exchange compared to healthy volunteers.

\section{P163 Propofol-induced inhibition of intestinal peristalsis involves enteric opioidergic pathways}

\section{MK Herbert, W Berg, P Holzer* and N Roewer}

Department of Anaesthesiology, University of Wuerzburg, Josef-Schneider-Strasse 2, D-97080 Wuerzburg, Germany; ${ }^{*}$ Department of Exp. and Clin. Pharmacology, University of Graz, Austria

Introduction: In recent years, the short acting propofol is increasingly used for sedation in intensive care units (ICU) patients. Inhibition of intestinal peristalsis is a major problem in those critically ill patients and an inherent feature of sedatives such as barbiturates or midazolam. Since little is known about the action of propofol on intestinal peristalsis, this study investigates the effect of propofol on the ileal peristaltic reflex in vitro.

Methods: lleal segments of adult guinea-pigs were mounted in silanized organ baths that contained oxygenated Tyrode solution $\left(30 \mathrm{ml}, 37^{\circ} \mathrm{C}\right)$. Prewarmed Tyrode solution was infused into the intestinal lumen, the infusion rate being $0.5 \mathrm{ml} \mathrm{min}{ }^{-1}$. The fluid passing the gut lumen was directed into a vertical outlet tubing which ended $4 \mathrm{~cm}$ (reflecting a pressure of $400 \mathrm{~Pa}$ ) above the fluid level of the organ bath. This arrangement caused gradual filling of the intestine. When the intraluminal pressure reached a threshold an aborally moving wave of circular muscle contraction, measured as a spike-like increase in intraluminal pressure, propelled the intraluminal fluid to leave the system and thus caused emptying of the segment. The pressure threshold for eliciting peristaltic waves (peristaltic pressure threshold - PPT) was used to quantify the effects of drugs on peristaltic activity. Inhibition of peristalsis was reflected by an increase of PPT. After registration of normal peristalsis the segments were exposed to propofol $(0.1-100 \mu \mathrm{M})$, which was administered cumulatively into the bath, i.e., to the serosal surface of at least 6 intestinal segments from 6 different guineapigs either alone, after application of vehicle (DMSO diluted with Tyrode solution), the $\mathrm{GABA}_{\mathrm{A}}$ receptor antagonist bicuculline (BIC, $10 \mu \mathrm{M})$, or the opioid receptor antagonist naloxone (NAL, $0.5 \mu \mathrm{M})$.

Results: Propofol (0.1-100 $\mu \mathrm{M})$ concentration-dependently increased the PPT, however, never caused complete inhibition of intestinal propulsion in the doses tested. While BIC $(10 \mu \mathrm{M})$ failed to prevent the inhibitory effect of propofol, the increase in PPT was absent after pretreatment with NAL $(0.5 \mu \mathrm{M})$.

Conclusions: Propofol inhibits intestinal peristalsis by increasing the PPT, but is less potent and effective compared to barbiturates or clonidine since it did not abolish peristaltic activity. The inhibitory effect of propofol seems not to be mediated through binding to $\mathrm{GABA}_{\mathrm{A}}$ receptors, but seems to involve enteric opioidergic pathways.

\section{P164 Clonidine concentration-dependently inhibits intestinal peristalsis in vitro}

\section{R Roth-Goldbrunner, MK Herbert, P Holzer* and N Roewer}

Department. of Anaesthesiology, University of Wuerzburg, Josef-Schneider-Strasse 2, D-97080 Wuerzburg, Germany, and *

Department of Exp. and Clin. Pharmacology, University of Graz, Austria

Introduction: The $\alpha$-adrenoceptor agonist clonidine provides a good alternative for sedation in patients in intensive care units (ICUs). Inhibition of intestinal transport, the development of ileus, and subsequently other complications are major side effects of sedatives used in ICU patients. This study examines whether clonidine exerts an inhibitory effect on intestinal peristalsis.

Methods: lleal segments of adult guinea-pigs were mounted in silanized organ baths that contained oxygenated Tyrode solution $\left(30 \mathrm{ml}, 37^{\circ} \mathrm{C}\right)$. Prewarmed Tyrode solution was infused into the intestinal lumen, the infusion rate being $0.5 \mathrm{ml} \mathrm{min}{ }^{-1}$. The fluid passing the gut lumen was directed into a vertical outlet tubing which ended $4 \mathrm{~cm}$ (reflecting a pressure of $400 \mathrm{~Pa}$ ) above the fluid level of the organ bath. This arrangement caused gradual filling of the intestine. When the intraluminal pressure reached a threshold an aborally moving wave of circular muscle contraction, measured as a spike-like increase in intralumal pressure, propelled the intraluminal fluid to leave the system and thus caused emptying of the segment. The pressure threshold for eliciting peristaltic waves (peristaltic pressure threshold - PPT) was used to quantify the effects of drugs on peristaltic activity. Inhibition of peristalsis was reflected by an increase of PPT. After registration of normal peristalsis the segments were exposed to clonidine $(1 \mathrm{nM}-100 \mu \mathrm{M})$, which was administered cumulatively into the bath, i.e., to the serosal surface 
of 6 intestinal segments from 6 different guinea-pigs either alone, after application of Tyrode solution (vehicle), or of the adrenoceptor antagonist yohimbine $(1 \mu \mathrm{M})$.

Results: Clonidine concentration-dependently increased the PPT. While Tyrode solution (vehicle) and 0.1-1 nM clonidine were without any effect on the PPT, $10 \mathrm{nM}-10 \mu \mathrm{M}$ clonidine caused an increase of PPT and complete abolition of peristalsis occurred after $100 \mu \mathrm{M}$ clonidine in 4 of 4 segments tested. Pretreatment with yohimbine $(1 \mu \mathrm{M})$ prevented clonidine $(0.1-100 \mathrm{nM})$ from having any inhibitory effect on PPT.

Conclusions: Clonidine concentration-dependently inhibits the ileal peristaltic reflex in vitro through adrenoceptors located in the intestine. It is assumed that clonidine also affects propulsive peristalsis in ICU patients and thus might contribute to other complications such as ileus and multiple organ failure in those patients.

\title{
P165 Early enteral nutrition after Pancreas Kidney Transplantation (PKTx) with enteral drainage (a pilot study in five patients)
}

\author{
D Agthé ${ }^{*}$, AR Mueller ${ }^{\dagger}$, H Full ${ }^{*}$ I Sauer ${ }^{\dagger}$, WO Bechstein ${ }^{\dagger}$, A Kahl', KJ Falke ${ }^{*}$ and P Neuhaus ${ }^{\dagger}$ \\ *Department of Anesthesiology and Operative Intensive Care and ${ }^{\dagger}$ Department of General and Transplant Surgery, Charité \\ Campus Virchow, Augustenburger Platz 1, D-13353 Berlin, Germany
}

Introduction: The use of early enteral nutrition, in particular formulas containing glutamine, arginine and omega-3fatty acids (immunonutrition), may have an impact on the post-operative course of critically ill patients. Previous research has demonstrated a decrease in complication rates, morbidity, and mortality. Little data exist, so far, about the use in pancreas-kidney-transplant recipients with enteral drainage. Because of the enteral anastomosis, most surgeons restrain from feeding the patients enterally, and postpone the start of enteral nutrition until at least five days after the procedure. No data is published on early enteral immunonutrition in this type of patient. The aim of this preliminary study, is to analyze the feasibility and complication rates of early enteral immunonutrition in pancreas-kidney-transplant recipients with enteral drainage.

Patients and Methods: Prospective analyzes of five patients after PKTx was performed. Nasogastric feeding with Impact ${ }^{\circledR}$ (Novartis, Switzerland), $25 \mathrm{cc} / \mathrm{h}$ was started immediately after surgery. This was continued for the first five days after PKTx. Additionally, patients recieve a parenteral standard nutrition program and substitution of fluids as needed to maintain diuresis. Further medication included propulsive agents (metoclopramide 20 mgs i.v. every $6 \mathrm{~h}$, cisapride $10 \mathrm{cc}$ p.o. every $6 \mathrm{~h}$ ) and immunosuppression (tacrolimus $0.05 \mathrm{mgs} / \mathrm{kg}$ or cyclosporine $4 \mathrm{mgs} / \mathrm{kg}$, ATG 5 $\mathrm{mgs} / \mathrm{kg}$, prednisolone $20 \mathrm{mgs} /$ day, mycophenolatmofetile $2 \mathrm{~g} /$ day). During the study for each patient basic demo- graphic and clinical characteristics including age, sex, SAPS II score, ICU and hospital mortality were recorded. The patients were screened for the occurrence of rejection, opportunistic infections and surgical complications.

Results: Early enteral immunonutrition was tolerated by all the patients. No surgical complications, especially no leakage at the enteric anastomosis, were observed. There was also no occurrence of transplant pancreatitis and a reduced incidence of bowel distention and vomiting. Except for one CMV-infection, no opportunistic infections or other infectious complications were observed. All patients developed rejection; rejection was mild and steroid-sensitive in four patients, one patient required OKT 3 treatment and tacrolimus rescue therapy.

Discussion and conclusion: The number of five patients is to small to make a valid conclusion and the results are rather descriptive. They show that early enteral feeding in this group of patients is possible without an increase in postoperative complications. Furthermore, early regeneration of mucosal gut barrier function may decrease infectious complications and improve patient outcome. In order to confirm this hypothesis further research should be performed to investigate the impact of this strategy. Variables like infection rates, surgical complications, and rejection, as well as length of ICU stay, morbidity and mortality will be analyzed.

\section{P166 Impact of carrier solution on biological insulin availability}

Z Rusavý, V Srámek, R Suchá, E Langhamerová, R Rokyta and O Topolcan*

Medical Dpt 1, *Medical Dpt 2, Charles Uni Hospital, 30460 Pilsen, Czech Republic

Objectives: When added into all-in-one (AIO) bags, total needs of exogenous insulin decrease compared to amounts given by perfusor. We studied the influence of carrier solution on insulin availability.
Materials and methods: A $20 \mathrm{ml}$ polyvinylchloride (PVC) syringe was filled either with saline or AIO solution aspirated from original $3 \mathrm{I}$ bags (Nutrimix, Brown, Melsungen, Germany). Then $8 \mathrm{IU}$ of insulin (Actrapid HM, Novo- 
Nordisk, DK) was added into syringes (calculated concen-

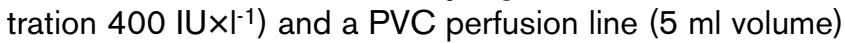
was filled. Baseline samples $(0.5 \mathrm{ml})$ for immunoreactive insulin assay (IRI) were taken. Perfusor rate was set at 2 $\mathrm{ml} \times \mathrm{hr}^{-1}$ and samples taken directly from the hose at 5,10 , $30,60,90$ and $210 \mathrm{~min}$, placed on ice and stored at $4^{\circ} \mathrm{C}$ before analysis. Fifteen sets of measurements were done both for saline and AIO solution. Values are presented as means $\pm S D$. MANOVA, ANOVA for repeated measures and paired T-test with Bonferroni correction were used when appropriate; $P<0.05$ was considered significant.

Results: Figure 1 shows IRI concentrations in both saline and $\mathrm{AIO}$ solutions during the experiment.

IRI differed significantly giving higher IRI yield in AIO solution compared to saline (MANOVA group by time effect $P<0.001)$. Changes in IRI concentration depending on time was seen in saline only (ANOVA time effect $P<0.001$ for saline; $P=0.26$ for $A I O)$. When separate time points for saline were analyzed major changes were seen at the very beginning of the experiment (a decrease from 206 \pm 34 at baseline to $152 \pm\left. 33 \mathrm{IU}^{*}\right|^{-1}$ at $\left.5 \mathrm{~min} ; P<0.001\right)$. The mean yield of IRI expressed as ratio of measured to calculated
Figure 1

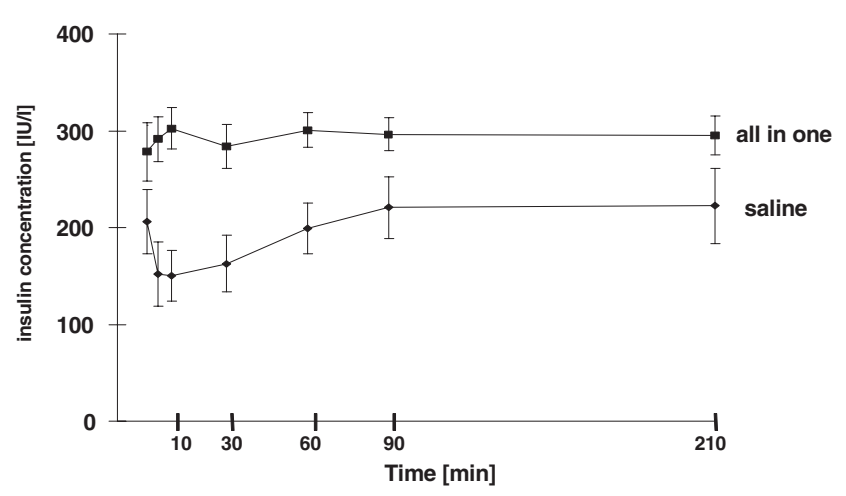

concentration at baseline was 0.52 for saline and 0.70 for AIO.

Conclusion: Insulin bioavailability in AIO solution is better than in saline with respect to the yield and stability.

\title{
P167 Delivery of parenteral nutrition by 'all in one bag' (AIO) versus single bottle system (SBS) in critically ill patients: a prospective randomized trial
}

\author{
C Ebener*, JM Harms`, B Thümmer†, R Buhl`, A Röhrborn†, PE Goretzki† and HD Röher ${ }^{\dagger}$ \\ ${ }^{*}$ Department of General Surgery, University of Regensburg, Franz Josef Strauß Allee 10, 94053 Regensburg; 'Department of \\ General and Trauma Surgery, Heinrich Heine University Moorenstraße 5, 40225 Düsseldorf; ”Department of Anaesthesiology, \\ Heinrich Heine University, Moorenstraße 5, 40225 Düsseldorf, Germany
}

Rationale: AIO has become an easy and practical way to deliver total parenteral nutrition (TPN) in patients on a regular ward. However no data are available yet concerning the use of AIO in critical ill patients. Here the SBS is still the gold standard.

Method: A prospective randomized trial was conducted in surgical patients who were expected to need TPN for more than five days after major surgery or trauma. Patients were randomized to receive for at least five days an isocaloric and isonitrogenic TPN delivered as AIO or as SBS. The TPN met the caloric requirements, which were determined by indirect calorimetry. Substrates delivered, numerous laboratory parameters, metabolic disturbances as a result of the nutrition, as well as expenses for equipment and manpower were documented daily. Forty patients are required by the protocol to complete the study.
Results: 37 patients have entered the study since 2/98. 3/37 refused to continue after the first day (drop out). 16 patients received $\mathrm{AIO}$ (86 days recorded) and 18 pat received SBS (101 days recorded). There was no difference in age, sex, diagnosis, risk factors between the treatment groups. Three patients died during the hospital course (18, 14 and 123 days after completing the study), all in the SBS group. Cause of death was in no case related to the TPN. The nutrition could be delivered without problems in both groups. Reduction of the TPN for hyperglycaemia was necessary only in one patient in each treatment group. Reduction of the TPN for hypertriglyceridaemia was necessary in one patient in each group. The SBS required more equipment e.g. infusion pumps and disposable infusion systems as well as more manpower e.g. to fix technical alarms.

Conclusion: AIB seems to be an useful alternative to SBS in critically ill and metabolically stable surgical patients. 


\section{P168 Glutamine-enriched parenteral nutrition during postoperative catabolic state}

O Ahlers, K Harndt, R Hodek, C Kietzmann, M Pettersson, K Ruland, Kv Fournier, P Neuhaus, K Falke and H Gerlach

Department of Anesthesiology and Intensive Care Medicine, Charité - Campus Virchow Clinic, Humboldt-University, 13344 Berlin, Germany

Background: Previous clinical studies led to the hypothesis that glutamine-enriched total parenteral nutrition (TPN) has beneficial effects on clinical course and on changes in the immune system of patients in postoperative catabolic state.

Patients and methods: 20 patients with esophageal carcinoma were enrolled in a controlled, prospective, randomized and double-blinded study after esophageal resection: 10 patients received glycyl glutamine-enriched $(0.15 \mathrm{~g}$ glutamine/kg/d) TPN (Glamin ${ }^{\circledR}$, Baxter Inc.) from day 2 postoperatively, 10 patients served as control (TPN without glycyl-glutamine: Vamin ${ }^{\circledR}$, Fresenius Inc). Clinical scores and immunological parameters were observed before and during the first 8 days after surgery. Lymphocyte subpopulations were measured using flow cytometry analyses (FACS-Flow ${ }^{\circledR}$, Becton Dickinson), plasma cytokine levels were determined using standard ELISA technique.
Results: No significant differences were found, neither in clinical nor in immunological parameters: SAPS II scores as well as GORIS scores showed similar courses in both groups and ICU-days did not differ. Postoperative alterations of the immune system, measured by plasma IL-6 and procalcitonin levels as well as changes in lymphocyte counts and T4-T8-ratio, were nearly similar.

Conclusion: A beneficial effect of glutamine-enriched TPN could not be found in our study population. Further investigations are necessary to find out if higher doses and/or other patient groups are able to demonstrate similar effects as shown in former studies.

Acknowledgement: Supported by Baxter Inc., Munich, Germany.

\section{P169 Muscular mass and subcutaneous fat changes according to duration of ICU hospitalization with corticosteroid and muscle relaxant intake}

M Moukas, AN Chalazonitis, P Dimou, A Patsalides, Cr Kouraziotis, E Xinis, C Mandragos, M Vasiliou and PK Behrakis Intensive Care Unit and Department of Radiology, Hellenic Red Cross Hospital, 1 Erythrou Stavrou str. Athens 11526, Greece

Introduction. In the present study we profit from the high U/S sensitivity and specificity in order to study the muscular mass (MM) and subcutaneous fat (SBF) changes during Intensive Care Unit hospitalization. In addition, we investigate the influence of the administration of muscle relaxants and corticosteroids.

Materials and methods: Thirty patients (19 male aged $51.1 \pm 14.15$ years hospitalized for $18.84 \pm 3.5$ days and 11 female aged $64.36 \pm 12.4$ years hospitalized for 19.18 \pm 4.6 days) entered the study. Clinical examination and computerized tomographic scan proved intracerebral hemorrhage in 14 of them and brain infarction in 16 of them. The patient's nutrition schedule included 30 $\mathrm{Kcal} / \mathrm{kg} /$ day with protein uptake of $1 \mathrm{~g} / \mathrm{kg} /$ day. Physiotherapy (passive movements of the limbs) was applied for 1 $\mathrm{h} /$ day in all patients. Muscle relaxation was applied to 9 patients with atracurium $(0.6-0.7 \mathrm{mg} / \mathrm{kg})$. The mean duration was $2.77 \pm 0.44$. Dexamethasone ( $24 \mathrm{mg} / \mathrm{d}$ iv) was applied to 14 patients for a mean duration of 4 days. Two ultrasonographic measurements were done for each patient; one on the first and another on the last hospitalization day in the ICU. Upper arm biceps MM and SBF thickness were assessed by M-mode ultrasonography using a $10 \mathrm{MHz}$ linear-array high resolution transducer. All scans were performed with the same ultrasonographer. $\mathrm{MM}$ and SBF thickness are expressed in $\mathrm{cm}$. MM, and SBF changes were calculated as differences of the 2 nd mea- surement minus the $1 \mathrm{st}$ and they were expressed as $\triangle \mathrm{MM}[\mathrm{cm}]$ and $\triangle \mathrm{SBF}[\mathrm{cm}]$, respectively.

Results: A significant loss of MM and SBF was observed in all patients included in the study. SBF loss (mean $\Delta \mathrm{SBF} \%=41.1 \pm 16.70)$ was significantly higher than corresponding $\mathrm{MM}$ reduction $(\triangle \mathrm{MM} \%=21.4 \pm 4.90)$. A significant correlation was found between $\Delta \mathrm{MM} \%$ and the duration of hospitalization ( $19 \pm 4$ days), $(r=0.62, P<0.05)$. On the contrary $\triangle \mathrm{SBF} \%$ was poorly correlated with the duration of the hospitalization ( $r=0.10, P>0.05)$. Neither $\triangle \mathrm{MM} \%$ nor $\triangle \mathrm{SBF}$ were correlated with patients' age. Patients who took corticosteroids $(n=5)$ showed a nonsignificantly higher percentage of MM loss in comparison to those who did not take corticosteroid ( $n=15$; corresponding $\triangle \mathrm{MM} \%$ : $22.1 \pm 5.22$ versus $20.0 \pm 4.83 \%$ ). Nevertheless, $\Delta \mathrm{MM} \%$ for the patients, who did not take muscle relaxation was slightly different $(20.4 \pm 4.53)$ from $\Delta \mathrm{MM} \%$ of patients who did not take corticosteroids or corticosteroids and muscle relaxants. A significantly higher reduction of $\mathrm{MM}$ was observed in patients who took corticosteroids and muscle relaxants in comparison to those who did not take either corticosteroids or muscle relaxants (corresponding $\Delta \mathrm{MM} \%$ : $23.8 \pm 4.90$ versus $19.8 \pm 4.47$ ). SBF did not differ significantly between patients who took and did not take corticosteroids (corresponding $\triangle \mathrm{SBF} \%$ : $47.5 \pm 26.47$ versus $40.6 \pm 18.91$ ). Not significant was also the SBF difference between those who took corticos- 
teroids and muscle relaxants ( $\triangle \mathrm{SBF} \%: 35.9 \pm 17.39)$ in comparison to those who did not take either corticosteroids or muscle relaxants ( $\triangle \mathrm{SBF} \%$ : $42.0 \pm 12.69$ ). $\triangle \mathrm{SBF} \%$ for patients who did not take muscle relaxation (43.2 \pm 15.95$)$ did not differ significantly from corresponding $\triangle S B F$ values of the other formatted groups.
Conclusions: Our study shows that MM loss is correlated with the duration of hospitalization as well as with the synchronous corticosteroids and muscle relaxants administration. On the contrary SBF, although decreased during the period of our study, is not statistically correlated with any of the studied factors.

\section{P170 Incidence of cortisol deficiency in patients with traumatic brain injury}

\section{MA Staber, DJ Cooper, O Montero and L Murray}

Intensive Care Department, The Alfred Hospital, Prahran, Melbourne 3181, Australia

Cortisol deficiency due to hypothalamic or pituitary injury has been uncommonly reported after traumatic brain injury (TBI), and the incidence is unknown. Diabetes insipidus after TBI, which usually reflects posterior pituitary injury, is however frequently recognized.

Blood cortisol concentrations are usually high in ICU patients - often 2-3 times greater than the normal morning peak values of $500 \mathrm{nmol} / \mathrm{l}$. Cortisol has a relatively short half-life and a permissive action on catecholamine efficacy, so cortisol deficiency could manifest rapidly, and cause hypotension.

In 80 patients with severe TBI (GCS <9), and in 12 controls (severe multi-trauma without TBI), we measured blood cortisol and ACTH concentrations on days 1,3 and 7 of ICU admission. Fifteen of 80 patients (19\%) had absolute cortisol deficiency with morning blood cortisol concentrations $<140 \mathrm{nmol} / \mathrm{l}$ (normal 140-500) and ACTH $<10 \mathrm{ng} / \mathrm{l}$ (normal $<65$ ) on days 3 or 7 of ICU admission. These patients had a mortality of $21 \%$ compared to $4 \%$ in the others. Twenty-four of the 80 patients $(30 \%)$ had blood cortisol concentrations $<250 \mathrm{nmol} / \mathrm{l}$. None of the controls had a blood cortisol concentration <140 nmol/l. TBI patents with blood cortisol concentrations $<250 \mathrm{nmol} / \mathrm{l}$ had greater elevation of intracranial pressure $32 \pm 45$ vs $9 \pm 23 \mathrm{~h}>20 \mathrm{mmHg}(P<0.05)$, and greater peak vasopressor doses $18 \pm 14$ vs $9 \pm 16 \mu \mathrm{g} / \mathrm{min}(P<0.05)$ than the others.

Absolute cortisol deficiency occurs during the first 7 days of ICU admission in 19\% of patients with severe TBI, and is likely to be of hypothalamic or pituitary origin. These patients had greater vasopressor doses suggesting that cortisol deficiency may contribute to vasopressor-resistant hypotension in patients with severe TBI.

\section{P171 Treatment of hypophosphataemia in critically ill patients with a two day dosing regimen}

\section{LJ Giles, A Jennings, B Ng, G Creed, L Gallard, D Pierre, R Beale and A McLuckie}

Department of Intensive Care, Guy's and St. Thomas' Hospital Trust, St. Thomas' Hospital, London, SE1 7EH, UK

Introduction: Standard phosphate replacement regimens $[1,2]$ are only $21-30 \%$ effective at correcting serum concentrations in severely hypophosphataemic critically ill patients, with $60-85 \%$ of patients redeveloping hypophosphataemia. We evaluated a two day regimen employing a rapid infusion rate.
Method: Fifty-two intensive care patients developing hypophosphataemia $(<0.8 \mathrm{mmol} / \mathrm{l})$ were evaluated. Intravenous phosphate doses on days 1 and 2 were based on the morning serum phosphate concentration: $0.8-0.6$ $\mathrm{mmol} / \mathrm{l}: 20 \mathrm{mmol}$ and $20 \mathrm{mmol}, 0.6-0.4 \mathrm{mmol} / \mathrm{l}: 40 \mathrm{mmol}$ and $20 \mathrm{mmol},<0.4 \mathrm{mmol} / \mathrm{l}: 50 \mathrm{mmol}$ and $20 \mathrm{mmol}$.

Table 1

\begin{tabular}{|c|c|c|c|c|c|c|c|c|c|}
\hline \multirow[b]{3}{*}{ Serum $\mathrm{PO}_{4} \mathrm{mmol} / \mathrm{l}$} & \multirow[b]{3}{*}{ Dose } & \multirow[b]{3}{*}{$\mathrm{N}$} & \multicolumn{5}{|c|}{ Efficacy } & \multicolumn{2}{|c|}{ Safety } \\
\hline & & & \multicolumn{2}{|c|}{$\begin{array}{l}\text { Mean (SD) serum } \\
\mathrm{PO}_{4}(\mathrm{mmol} / \mathrm{l})\end{array}$} & \multicolumn{3}{|c|}{$\begin{array}{l}\text { Patients \% successfully } \\
\text { corrected }(\geq 0.8 \mathrm{mmol} / \mathrm{l})\end{array}$} & \multicolumn{2}{|c|}{$\begin{array}{l}\text { Mean }(\mathrm{SD}) \text { change in } \\
\text { ionised } \mathrm{Ca}(\mathrm{mmol} / \mathrm{l}) \text { from } \\
\text { pre-treatment value }\end{array}$} \\
\hline & & & $\begin{array}{l}\text { dose } 1 \\
+24 \mathrm{~h}\end{array}$ & $\begin{array}{l}\text { dose } 2 \\
+24 h\end{array}$ & $\begin{array}{l}\text { dose } 1 \\
+24 \mathrm{~h}\end{array}$ & $\begin{array}{c}\text { dose } 2 \\
+24 \mathrm{~h}\end{array}$ & $\begin{array}{l}\text { dose } 2 \\
+48 h\end{array}$ & $\begin{array}{l}\text { dose } 1 \\
+24 \mathrm{~h}\end{array}$ & $\begin{array}{l}\text { dose } 2 \\
+24 \mathrm{~h}\end{array}$ \\
\hline $0.8-0.6$ & $20: 20$ & 35 & $0.9(0.2)$ & $1.1(0.4)$ & $66 \%$ & $71 \%$ & $74 \%$ & $-0.06(0.11)$ & $-0.07(0.17)$ \\
\hline $0.6-0.4$ & $40: 20$ & 13 & $0.8(0.2)$ & $0.8(0.2)$ & $46 \%$ & $53 \%$ & $69 \%$ & $+0.03(0.16)$ & $-0.04(0.12)$ \\
\hline$<0.4$ & $50: 20$ & 4 & $0.8(0.2)$ & $0.9(0.3)$ & $25 \%$ & $75 \%$ & $75 \%$ & $-0.02(0.03)$ & $+0.01(0.05)$ \\
\hline
\end{tabular}


Sodium phosphate $(0.1 \mathrm{mmol} / \mathrm{ml})$ was infused at 10 $\mathrm{mmol} / \mathrm{h}$. Serum phosphate and ionised calcium concentrations and calcium phosphate product were determined pre-infusion, immediately post-infusion and $6 \mathrm{~h}$ post-infusion for each dose and after $24 \mathrm{~h}$. Twenty-two patients were receiving CRRT.

Results: See Table 1.

Only seven calcium phosphate product values were slightly raised (range: $4.9-5.7 \mathrm{mmol}^{2} / \mathrm{I}^{2}$ ). Twenty-two patients had ionised calcium concentrations of $\leq 1.05$ $\mathrm{mmol} / \mathrm{l}$ on occasions during the regimen but the extent of the hypocalcaemia was mild (mean [SD] ionised Ca: 0.99
(0.07) range: $0.68-1.05 \mathrm{mmol} / \mathrm{l}[\mathrm{n}=50]$ ) and usually transient. Furthermore the degree of change in ionised calcium concentrations from pre-treatment values for all patients was not significant (see Table 1). No clinical evidence of hypocalcaemia was noted.

Conclusion: The two day intravenous phosphate regimen described is safe and highly effective at correcting hypophosphataemia in critically ill patients.

References:

1. Perreault MM, Ostrop NJ, Tierney MG: Ann Pharmacother 1997, 31:683-688.

2. Clark CL, Sacks GS, Dickerson RN et al::Crit Care Med 1995, 23:1504-1511.

\section{P172 Hypoglycemia masquerading as acute psychosis in young age}

P Klemen, Š Grmec and D Cander

EMS-PHU ZD Maribor, UI. Talcev 9, 2000 Maribor, Slovenia

Introduction: The classic presentation of hypoglycemia includes tachycardia, anxiety, extreme hunger, tremor, palpitations, pallor, weakness and an altered level of consciousness. The hypoglycemic patient's presentation may also lead to suspect another condition such as cerebrovascular accident, seizure, head injury or acute psychosis.

A clinical study was done to determine whether there is a correlation between the manifestations of hypoglycemia and the patient's age.

Methods: We studied 191 prehospital patients in the period from February 1998 to September 1999. For each patient we studied: the level of blood glucose, age and clinical manifestations of hypoglycemia.

Results: 99 (51.4\%) patients presented with coma, 24 patients $(12.6 \%)$ presented with focal neurological deficit or seizure activity, $56(29.2 \%)$ were confused and 12 $(6.8 \%)$ presented as acute psychosis.

We found out that there existed a statistically important difference in patient's age between clinical manifestations of hypoglycemia (Table).
Table

Manifestations of Hypoglycemia

Age (Years)

Coma

$51.3 \pm 19.2$

Focal Neurol. Findings or Seizure

$58.3 \pm 11.3$

Confusion

$61.2 \pm 17.3$

Psychosis

$34.2 \pm 6.3$

Conclusion: In our study we found out that psychosis as a manifestation of hypoglycemia presents in significantly younger patients than other manifestations (Student t-test $<0.05$ ). In elderly patients hypoglycemia presents more often with focal neurological deficit, seizure, confusion and coma. Our cases illustrate the importance of considering hypoglycemia in all patients who present with alterations in mental status even when the clinical findings seem to be explained initially by other etiologies. Emergency physicians must be aware of such a presentation of hypoglycemia and the need for rapid testing, since testing is easily performed and therapy is most often curative with a good outcome.

\section{P173 Plasma Cl:Na ratio: a simple alternative to Stewart's Strong lon equation for detection of elevated lactate or unmeasured anions in metabolic acidosis}

\section{S Skellett, A Mayer, A Durward, S Tibby and IA Murdoch}

Guy's Hospital, London SE1 9RT, UK

Introduction: There has been a resurgence of interest in the interpretation of acid base abnormalities using Stewart's Strong lon Theory [1], central to which is the solving of a complex quadratic equation that accurately quantifies the contribution of both organic and inorganic anions to $\mathrm{pH}$. In accordance with this theory, we hypothesised that an increase in organic anions (lactate and unmeasured anions) during metabolic acidosis would cause a compensatory fall in inorganic chloride thus preserving electroneutrality. The aim of this study was to determine if this compensatory hypochloraemia, expressed as a fall in the 
$\mathrm{Cl}: \mathrm{Na}$ ratio, could be a useful method to detect these elevated organic anions in patients with metabolic acidosis.

Patients and methods: Paired blood samples were taken on admission and at $24 \mathrm{~h}$ from 341 patients (median weight $8 \mathrm{~kg}, \mathrm{IQ}$ range $3-15$ ) admitted to a regional PICU, from which serum electrolytes, lactate and blood gases were measured and the strong ion gap calculated to quantify unmeasured anions (UMA). A metabolic acidosis was defined as being present if the base deficit (BD) was $\geq 5 \mathrm{mmol} / \mathrm{l}$ and was chosen rather than $\mathrm{pH}$ since it better defines the metabolic component of an acidosis [2].Using the above criteria for acidosis, 136 samples were selected and further divided into two subgroups using a $\mathrm{Cl}: \mathrm{Na}$ ratio $\leq 0.74$ as the cutoff value and compared for acid base parameters, lactate and UMA concentrations. The cut off value of 0.74 represents the 1 st quartile for $\mathrm{Cl}: \mathrm{Na}$ ratio derived from analysis of the 341 paired samples (median 0.76 , IQ range $0.74-0.78$ ). This approach was necessary since there are no normal values for $\mathrm{Cl}: \mathrm{Na}$ ratio.

Results: In the episodes characterised by a BD $\geq 5 \mathrm{mmol} / \mathrm{l}$ $(n=136)$, the blood lactate and UMA concentrations were significantly greater in the subgroup with a $\mathrm{Cl}: \mathrm{Na}$ ratio $\leq 0.74$ despite having similar conventional acid base parameters $\left(\mathrm{pH}, \mathrm{pCO}_{2}\right.$ and $\left.\mathrm{BD}\right)$.

Conclusion: A low plasma Cl: $\mathrm{Na}$ ratio $(\leq 0.74)$ is a simple alternative test for detecting the presence of abnormal elevations in lactate $(>2.4 \mathrm{mmol} / \mathrm{l})$ or UMA $(>4.3 \mathrm{mmol} / \mathrm{l})$ concentrations in patients with metabolic acidaemia. This obviates the need to solve the complex quadratic equa-
Table 1

\begin{tabular}{|c|c|c|c|}
\hline & $\begin{array}{c}\mathrm{Cl}: \mathrm{Na} \leq 0.74 \\
(\mathrm{n}=35)\end{array}$ & $\begin{array}{c}\mathrm{Cl}: \mathrm{Na}>0.74 \\
(\mathrm{n}=101)\end{array}$ & $\begin{array}{c}P \text { - value } \\
\text { Mann- } \\
\text { Whitney }\end{array}$ \\
\hline $\mathrm{pH}$ & $7.22(7.13-7.31)$ & $7.28(7.21-7.32)$ & 0.2 \\
\hline $\mathrm{pCO}_{2}(\mathrm{kPa})$ & $4.4(3.7-6.7)$ & $4.6(3.9-5.3)$ & 0.9 \\
\hline $\mathrm{BD}(\mathrm{mmol} / \mathrm{l})$ & $10(6-13)$ & $7(6-11)$ & 0.1 \\
\hline $\mathrm{LAC}(\mathrm{mmol} / \mathrm{l})$ & $7(2.2-9)$ & $1.3(0.8-3.6)$ & $<0.001$ \\
\hline UMA (mmol/l) & $12(8-15)$ & $3(-1-7)$ & $<0.001$ \\
\hline
\end{tabular}

Table 2

ROC curve values for $\mathrm{Cl}: \mathrm{Na}$ ratio $\leq \mathbf{0 . 7 4}$. Values in $\mathrm{mmol} / \mathrm{L}$

\begin{tabular}{lcccc}
\hline & $\begin{array}{c}\text { Cut-off } \\
\text { value }\end{array}$ & \multicolumn{4}{c}{ Sensitivity } & Specificity & $\begin{array}{c}\text { AUC } \\
(95 \% \mathrm{Cl})\end{array}$ \\
\hline Lactate & $>2.4$ & 72 & 65 & $0.75(0.64-0.85)$ \\
UMA & $>4.3$ & 86 & 64 & $0.82(0.75-0.9)$ \\
Lactate + UMA & $>12$ & 89 & 81 & $0.86(0.8-0.93)$ \\
\hline
\end{tabular}

tions required for calculation of UMA using the Strong lon Theory of Stewart.

References:

1. Stewart: Can J Physiol Pharmacol 1983, 61:1444.

2. Balasubramanyan: N: Crit Care Med 1999, 8:1577-1581.

\section{P174 Effects of angiotensin II on the renal excretion of urodilatin in the isolated rat kidney}

\section{Heringlake*, K Wagner*, L Bahlmann*, S Klaus*, J Schumacher*, H Pagel† and P Schmucker*}

${ }^{*}$ Klinik für Anaesthesiologie, and +Institut für Physiologie, Medizinische Universität Lübeck, D - 23538 Lübeck, Germany

Background: The kidney-derived peptide urodilatin has been suggested to be important in the regulation of natriuresis and diuresis. This hormone has been used clinically as a drug for the treatment of incipient renal failure in cardiac surgery patients and is known to be increased in these patients if renal function is preserved [1]. However, the mechanisms involved in the regulation of the production of urodilatin are far from being clear. This study was designed to determine, if Angiotensin II may influence the renal excretion of urodilatin $\left(\mathrm{V}_{\mathrm{URO}} \mathrm{U}\right)$.

Materials and methods: Isolated kidneys from SpragueDawley rats were perfused for $180 \mathrm{~min}$ with a pressure of $100 \mathrm{mmHg}$ in a recirculating perfusion system. After an equilibration time of 90 min (baseline-period), the following drugs were added to the perfusion medium: Angiotensin II (All: $n=5)$; Losartan (LOSA: $n=5$ ), and both drugs combined (LOSA-All: $n=5$ ) and the kidneys were perfused for another $90 \mathrm{~min}$ (intervention-period). Five kidneys served as controls (CON). Measurements were performed every $30 \mathrm{~min}$. Data of both observation periods were averaged (baseline: $0-90 \mathrm{~min}$; intervention: 91-180 $\mathrm{min}$ ); baseline data were normalized to $100 \%$. Statistical analyses were performed with ANOVA and Fisher's PLSD.

Results: Data for the intervention period are given in percent of average baseline values (Table 1). RVR: renal vascular resistance; $\mathrm{VU}$ : urine flow; $\mathrm{V}_{\mathrm{Na}} \mathrm{U}$ : urinary excretion of sodium; GFR: glomerular filtration rate; $\mathrm{V}_{\mathrm{URO}} \mathrm{U}$ urinary excretion of urodilatin.

Conclusions: These observations suggest that $\mathrm{V}_{\mathrm{URO}} \mathrm{U}$ is affected -directly or indirectly - by vasoconstrictory concentrations of Angiotensin II and may help to explain recent findings on the regulation of the renal excretion of urodilatin; especially the positive relationships of $\mathrm{V}_{\mathrm{URO}} \mathrm{U}$ with plasma sodium [2] and renal perfusion pressure [3]. 
Table 1

\begin{tabular}{llllll}
\hline & RVR & $\mathrm{VU}$ & $\mathrm{V}_{\mathrm{Na}} \mathrm{G}$ & $\mathrm{GFR}$ & $\mathrm{V}$ \\
\hline CON & $102.2 \pm 2.4$ & $187.2 \pm 13.9$ & $258.7 \pm 24.7$ & $97.2 \pm 4.3$ & $61.1 \pm 13.9$ \\
All & $164.3 \pm 11.3^{*}$ & $112.8 \pm 17.1^{\star}$ & $134.1 \pm 23.5^{\star}$ & $72.9 \pm 4.7^{\star}$ & $21.9 \pm 2.9^{\star}$ \\
LOSA & $115.3 \pm 8.8$ & $197.3 \pm 35.2$ & $250.9 \pm 51.5$ & $95.8 \pm 13.1$ & $72.3 \pm 17.1$ \\
LOSA-All & $112.6 \pm 13.9$ & $152.6 \pm 24.9$ & $201.7 \pm 42.4$ & $89.8 \pm 7.3$ & $38.2 \pm 14.9$ \\
\hline
\end{tabular}

*Denotes a significant difference $(P<0.05)$ vs control.

\section{References:}

1. Sehested et al.: J Thoracic Cardiovasc Surg. 114:666-671.

2. Drummer et al.: Am J Physiol 262:F744-F754.

3. Heringlake et al.: Am J Physiol 277:F347-F351.

\section{P175 Plasma endothelin-1 and natriuretic peptide levels and antioxidant capacity of heart transplanted} patients

I Olejarova, K Danova, H Minarova, V Fischer, V Rendekova, J Fabian and I Pechan

Slovak Institute of Cardiovascular Diseases, Pod Krasnou Horkou 1, Bratislava, Slovak Republic

There is evidence that a transplanted heart does not provide the recipient with functionally normal cardiac function and many differences in resting haemodynamics and alterations in electrophysiology and neurohumoral disbalance can be seen. Therefore, the plasma levels of functionally important metabolites - endothelin-1 (ET-1) as well as A- and B-type natriuretic peptides (ANP and $B N P)$ - are released into the blood bed in response to fluid retention and vasoconstriction in heart disorders. Besides these haemodynamically-interesting parameters, in various intervals after the heart transplantation $(\mathrm{HTx})$, the actual endogenous antioxidant capacity of patients was studied. Before HTx and successively three times during the first week and then weekly during the first month after HTx, seven transplanted patients were carefully clinically and biochemically investigated. In their blood plasma (or erythrocytes) the total antioxidant status (TAS), activities of superoxide dismutase (SOD) and glutathione peroxidase (GPx) as well as plasma levels of thiobarbituric acid reactive substances (TBARS), ET-1, ANP and BNP were estimated.

During the whole investigation period the gradual decrease of TAS was noted. Compared with the pretransplant levels, a gradual decrease of plasma levels of ET-1 and successive rise in ANP and BNP levels were observed, especially during the first week after the HTx. In conclusion, we have shown that HTx-patients demonstrated decreased total antioxidant capacity and characteristic movements of plasma levels of analysed peptides reflects the response of the cardiovascular system to the haemodynamically different situation.

P176 Management and outcome of symptomatic hyponatremia in emergency department

\section{J Kato and Y Tokuda}

Okinawa Chubu Hospital, 208-3 Miyazato, Gushikawa city 902, Okinawa, Japan

Introduction: Severe symptomatic hyponatremia was thought to be extremely dangerous. It is recommended that acute hyponatremia should be treated without delay and rapidly at a rate of at least $1 \mathrm{mmol} / \mathrm{l} / \mathrm{hour}$. Symptomatic hyponatremia was said to lead to death or permanent brain damage. However, some authors did not support quick correction and suggested that most deaths were caused by underlying diseases. We evaluated the clinical outcome of cases with symptomatic hyponatremia in terms of different management strategies in the emergency department.

Method: In the emergency outpatient department of a community hospital, Okinawa, Japan, we retrospectively collected adult cases of symptomatic hyponatremia (serum sodium <130mmol/l) from April 1995 to October 1999. Etiology, treatment and clinical outcomes were evaluated. Neurological sequelae were assessed in all cases.

Result: Eighty-two cases of hyponatremia were determined (mean age, 54 years old, 48 males and 34 females). The mean sodium level was $118 \mathrm{mmol} / \mathrm{l}$ (the lowest $104 \mathrm{mmol} / \mathrm{l})$. There were 45 cases with consciousness disturbance and 33 cases with seizure. Fifty-three cases were acute and 29 chronic. Underlying etiology included water intoxication, SIADH and hypoadrenalism. Death occurred in four cases and all were caused by underlying diseases. Thirty-five cases were treated with isotonic saline, 15 cases with hypertonic saline, and 32 
cases with no active fluid treatment. Although correction rate for hyponatremia was not rapid $(<0.6 \mathrm{mmol} / / / \mathrm{h})$ for all cases, there were no cases of mortality and neurological sequelae from hyponatremia.

Conclusions: Slow correction of serum sodium was not complicated with permanent brain damage from acute symptomatic hyponatremia. Rapid correction is not neces- sary for treatment of symptomatic hyponatremia just because the serum concentration is extremely low.

\section{References:}

1. Sterns RH: Severe symptomatic hyponatremia: treatment and outcome. Ann Intern Med 1987, 107:656-664.

2. Ayus JC, Krothapalli RF, Arieff Al: Treatment of symptomatic hyponatremia and its relation to brain damage. $N$ Engl J Med 1987, 317:1190-1195

\section{P177 Variation of early auditory evoked potentials (EAEP) in severe hyponatremia}

\section{Dusson, T Van der Linden, P Cabaret, X Fournet, P Gallois, G Forzy, YM Robin and B Lepoutre}

Intensive care unit, CH Saint-Philibert, LOMME 59462, France

Severe hyponatremia induces neurological symptoms in connection with its rate of appearance. The aim of this study was to measure the variations of EAEP during hyponatremia and to determine its prognostic interest.

Patients and method: 65 patients (44 women and 21 men, mean age $67.3 \pm 15.7$ years) with hyponatremia $<125 \mathrm{mmol} / \mathrm{l}$ at their admission to the emergency room or intensive care unit, were included. The EAEP (monaural stimulation by alternative clicks of $0.1 \mathrm{~ms}$ at $90 \mathrm{Db}$ ) were recorded daily until hyponatremia correction was achieved (250 records). EAEP parameters (I, III and V peaks latency, I-III, III-V and I-V intervals) were studied according to the level and the mechanism of hyponatremia, and according to the neurological symptomatology and survival of the patients.
Results: The mean value of the initial natremia was $118 \pm 7$ $\mathrm{mmol} / \mathrm{l}$, by dilution in $78 \%$ of cases, with neurological signs in $69 \%$. Correction speed was $4.8 \pm 2.2$ days. Fifteen patients died but no cases of centropontine myelinolysis occurred. There was a linear correlation between natremia decrease and brainstem conduction lengthening (III and $\mathrm{V}$ peaks, intervals, $P<10^{-4}$ ). These variations were corrected by the normalization of natremia $\left(P<10^{-5}\right)$. Hyponatremia was associated with a lengthening of the I-V interval in $74.5 \%$ of patients. The mechanism of the hyponatremia, the presence of neurological signs or the patient outcome had no effect on EAEP parameters.

Conclusion: Severe hyponatremia must be considered a cause of EAEP lengthening. EAEP evaluation does not constitute a prognostic factor of hyponatremia.

\section{P178 Combined central diabetes insipidus and cerebral salt wasting after craniotomy}

\section{TF Loh, WRW Lee, YCK Goh and WT Seow}

Department of Paediatric Medicine and Department of Neurosurgery, KK Women's and Children's Hospital, 100 Bukit Timah Rd, Singapore 229899

Introduction: Sodium disturbances and polyuria in children after craniotomy for intracranial lesions are not uncommon. Diabetes insipidus (DI) of central origin is often cited as a cause of high serum sodium [1] while cerebral salt wasting (CSW) is a recognised cause of hyponatraemia in these patients [2]. DI and CSW can both result in polyuria; patients with central DI suffer from free water loss while patients with CSW have severe salt loss. Both syndromes may occur at various times in the postoperative period. A combination of the above mentioned diseases could occur in the clinical setting and this can lead to difficulty in diagnosis and cause problems in maintaining normal fluid and sodium status. Proper differentiation is essential, as the treatment for each entity is different. CSW and DI in the same clinical setting have not been previously described in the paediatric age group. We report two cases of combined DI and CSW in the immediate postoperative period.

Case reports: Case 1. CXH is a 10 year old girl who presented with 1 month's history of visual and gait disturbances.
MRI of the brain showed a large suprasellar tumour with hydrocephalus. A ventriculo-peritoneal shunt was inserted immediately. She needed two operations to completely excise the tumour. Histology showed pilocytic astrocytoma. Postoperatively, she developed evidence of central diabetes insipidus and required intravenous pitressin before urine output was successfully controlled. She remained stable till the fifth postoperative day (POD) when she developed hyponatraemia that was very resistant to treatment despite hypertonic saline replacements. She was also clinically dehydrated. Urine sodium was $296 \mathrm{mEq} / / / / \mathrm{l}$ and $\mathrm{FeNa}$ was 10.8 . With aggressive saline replacements, hyponatraemia was corrected and kept within normal limits on a regimen of enteral feeds and intravenous saline replacements according to urine output and sodium measurements. Fludrocortisone was started. After tenth POD, urinary sodium began to decline consistently below $150 \mathrm{mEq} / / \mathrm{l}$ by the fifteenth POD and less than $70 \mathrm{mEq} / / \mathrm{l}$ by a month POD.

Case 2. NWS presented at 1.5 months of age with rhinorrhea and nasal mass. MRI showed a nasal mass with 
intracranial extension. Consent for surgery was not given until a year and a half later. By that time, a repeat MRI showed the tumour had extended into the left orbit and ethmoid sinus. A lateral rhinotomy, craniotomy with tumour excision and craniofacial reconstruction was performed. Histology revealed a low-grade nasal glioma. Postoperatively, he developed central diabetes insipidus requiring intravenous pitressin before urine output was controlled. However, serum sodium continued to drop even though urine output did not change significantly. On the second POD, the child developed a generalised tonic clonic seizure that was aborted with intravenous Valium and dilantin. Computer tomography scan of the head showed residual tumour in the left orbit and suprasellar region, evidence of CSF leak but no haemorrhage or cerebral oedema. Central venous pressure had dropped to $+3 \mathrm{cmH}_{2} \mathrm{O}$. Serum and urine sodium was $117 \mathrm{mEql} / \mathrm{l}$ and $176 \mathrm{mEql} / \mathrm{l}$. FeNa was 9.6. Hyponatraemia was corrected gradually with normal saline and hypertonic saline replacements. However, urinary sodium levels continued to rise over the ensuing days, reaching a peak of $295 \mathrm{mEql} / \mathrm{l}$ on the sixth POD and there was massive solute diuresis. He required aggressive replacements with hypertonic saline to keep serum sodium within limits. However, urinary sodium persisted between 120-135 mEql// thereafter and on the tenth POD, fludrocortisone was started at $10 \mu \mathrm{g} / \mathrm{kg} /$ day. Subsequently, urinary sodium dropped to $56 \mathrm{mEql} / \mathrm{l}$ two weeks after surgery and remained below
$30 \mathrm{mEq} / / \mathrm{l}$ after three weeks post surgery. Intravenous pitressin was successfully weaned off and the patient started on intranasal DDAVP.

Discussion: CSW syndrome is characterised by hyponatraemia $(<130 \mathrm{mEq} / \mathrm{l})$, dehydration, and inappropriate urinary sodium loss that responds to fluid and saline replacements. Osmotic diuresis often accompanies this syndrome. The main feature of central DI is massive diuresis of dilute urine with low sodium content thus resulting in hypernatraemia and dehydration. Both clinical states are characterised by diuresis but in CSW, hyponatraemia is present while in $\mathrm{DI}$, the patient experience hypernatraemia. In the neurosurgical intensive care setting, ensuring sodium homeostasis is important to maintain an environment without major flux in osmolality. CSW may develop in patients with an established diagnosis of DI. Early recognition can be achieved by measuring osmolality and sodium in the plasma and urine as well as plasma ANP levels. The cornerstone of CSW management remains replacing sodium and water loss with normal or hypertonic saline. In our experience, mineralcorticoid therapy appears to be an effective adjunct [3].

\section{References:}

1. Lugo $\mathrm{N}$ et al.: J Ped Endo Met 10:633-639.

2. Harrigan MR et al:: Neurosurgery 1996, 38:152-160.

3. Ishikawa S et al:: Ann Int Med 1987, 106:187-191.

\section{P179 Changes in the C-type Natriuretic peptide (CNP) and NO derivatives in the plasma and CSF of the patients with subarachnoid hemorrhage (SAH)}

K Ikeda, T Ikeda, T Onizuka, H Terashi, T Nakanishi*, T Fukuda* and S Endo ${ }^{\dagger}$

Division of Critical Care and Emergency Medicine and ${ }^{*}$ Department of Neurosurgery, Hachiouji Medical Center of Tokyo Medical University; ${ }^{\dagger}$ Department of Emergency Medicine, Iwate Medical University, Japan

The aim of this study was to determine a role of CNP and NO for cerebral vasospasm following subarachnoid hemorrhage (SAH). Both of them are endothelium-derived relaxing factor (EDRF), and CNP exists in the brain as a cerebral vasodilator. We conducted a one week monitoring of CNP and NOx levels in the plasma and cerebrospinal fluid (CSF) in 26 patients who received clipping operation within $24 \mathrm{~h}$ after the occurrences of $\mathrm{SAH}$, and classified to the Group A (angiographical spasm +), and Group B (angiographical spasm -). And we examined CNP and NO, levels in the CSF of the patients who received spinal anesthesia for a small operation as a reference patients.

Results: CNP levels in the CSF on day 1 of hospitalization was higher than that of the reference patients and the level decreased significantly on day 7 , but we did not observe any significant difference between the groups. CNP levels in the plasma did not change.
NOx levels in the CSF in the week following SAH were higher than the levels of the reference patients, and the level in Group A was higher than in Group B on day 1. Only in Group A did NOx levels decrease significantly from day 3. NOx levels in the plasma did not change significantly.

Conclusions: CNP levels in the CSF were high in the acute phase following $\mathrm{SAH}$, while plasma CNP levels were maintained at a constant. But any specific role of CNP for cerebral vasospasm was not indicated from our findings.

We considered that NOx in the CSF has a role as an inhibitor for cerebral vasospasm following $\mathrm{SAH}$, because NOx levels decreased significantly only in the vasospasm group, while plasma NOx levels did not change significantly. 


\section{P180 Acute weakness in the ICU-electrophysiological and pathological findings}

J Manák*, Z Lukáš†, M Schreiber ${ }^{\ddagger}$, K Kunc ${ }^{\ddagger}$, H Matulová ${ }^{\ddagger}$, F Pára ${ }^{\ddagger}$, L Sobotka* and Z Zadák

${ }^{*}$ Dept. Metabolic Care, University Hospital Hradec Králové, Czech Republic; ${ }^{\dagger}$ Dept. Pathology II, Childrens Hospital, University

Brno, Czech Republic; ₹Dept. Neurology, University Hospital Hradec Králové, Czech Republic

Objective: To describe electrophysiological and histopathological findings in patients with acute weakness, which developed in the ICU during the course of critical illness.

Materials and methods: During a 15 month period, all patients with clinical signs of acute onset quadruparesis were studied prospectively. In nine critically ill patients of mean age 55 (21-78) years, clinical examination, electrophysiological studies (conduction studies of peripheral motor and sensory nerves, needle electromyography, repetitive stimulation) and skeletal muscle biopsies were performed. None of the patients had any neuromuscular abnormality in anamnesis.

Results: Clinical examination: all of our patients had signs of quadruparesis and generalized hypo- or areflexia of tendon reflexes and prolonged dependence on the ventilator. Electrophysiology: compound muscle action potentials (CMAP) were markedly reduced (mean $18 \pm 15 \%$ of normal values) or totally absent in all patients. Sensory nerve action potentials (SNAP) were reduced or absent in six patients, only two patients had normal SNAP. Motor nerve conduction velocities were normal or slightly reduced in six patients, two patients had significantly lower motor nerve conduction velocities. Sensory nerve conduction velocities were normal, there was only slight reduction in one patient. Needle electromyography showed fibrillations and positive sharp waves in eight patients. No pathology in repetitive stimulation was found. Histopathology: all skeletal muscle biopsies showed pathologic alterations. Fiber diameters varied from atrophy to normal. In muscle fibers, internal nuclei, fiber splitting and focal regressive changes with increased activity of lysosomal acid phosphatase were found. In 50\% of cases, regenerating fibers were present. Endomysial fibrosis was common. All the changes were classified as myogenic, no convincing signs of neurogenic atrophy were noticed. One patient revealed disuse atrophy.

Conclusions: We found electrophysiologic signs of axonal sensory-motor polyneuropathy in all our patients. In spite of that, no neurogenic changes in the muscle were found in the biopsies, the changes in skeletal muscles had a myopathic pattern. These data suggest that in acute quadruplegia of the critically ill, both the nerve and the muscle are affected simultaneously.

Acknowledgement: This study was supported by grant IGA MZ CR No. NB 5197-3.

\section{P181 A prospective study of the incidence of critical illness polyneuropathy}

\section{Segura, ME Arteaga and F Delgado}

Unidad de Medicina Critica, Departamento de medicina, Hospital Medico Quirurgico (ISSS), Alameda Juan Pablo II, San

Salvador, El Salvador

Introduction: Critical illness polyneuropathy (CIP) has been associated with sepsis and multiorgan dysfunction syndrome (MODS). Studies have reported an incidence of CIP that oscillated among 21-81.8\% [1,2,3].We conducted this study to assess the incidence of CIP in our intensive care unit.

Setting: Polyvalent ICU.

Method: During the period of 05/01/1999 to 10/30/1999 359 patients entered our unit, from this group 154 needed mechanical ventilation for more then five days. The APACHE II score and GORIS score of multiorgan failure was evaluated. When they were transferred from the unit EMG study was realized according to the protocol published before [1].

Patients with a history of polyneuropathy, chronic renal failure, diabetes mellitus, chronic alcoholism and thus patients with polytraumatism of lower extremity, in which it was not possible to realize the study, were excluded.

Results: See Table.

Our results show a smaller incidence (6.6\%) of CIP than other studies $[1,2]$.These studies had a major proportion of patients with neurotrauma and polytrauma and our sample was only $13 \%$. Nevertheless, we can observe a score of FMO larger than or equal to 5, and a more extended mechanical ventilation tim,e which can also explain the difference that was found.

References:

1. Leizten FSS, De Weere AW: Critical illness polyneuropahy in multiorgan dysfunction syndrome and weaning from the ventilator. Intensive Care Med 1996, 22:856-861.

2. Mohr M: Effects of early treatment with inmunoglobulin on critical illness polyneuropathy following multiple organ failure and gran negative sepsis. Intensive Care Med 1997, 23:1144-1149.

3. Berek K, Maugreiterj: Polyneuropathy in critically ill patients a prospective evolution. Intensive Care Med 1996, 33:849-855. 


\begin{tabular}{|c|c|c|c|c|c|c|}
\hline Study & Time & $\mathrm{N}$ & Mechanical ventilation & APACHE & GORIS SCORE & CIP \\
\hline 1. Segura V & 4 months & 15 & 14.1 days & 25.8 & 4.6 & $6.6 \%$ \\
\hline 2. Berek K & 1 year & 23 & - & - & - & $81.8 \%$ \\
\hline 3. Leizten $\mathrm{F}$ & 18 months & 38 & 33.6 days & 21.9 & 5.3 & $47 \%$ \\
\hline MOHRM & 2 years & 62 & 23 days & 22 & 5 & $21 \%$ \\
\hline
\end{tabular}

\section{P182 Neurosurgical patients admitted to intensive care units (ICU) exhibit a systemic inflammatory response}

DF McAuley†, K McCallion, DW Harkin, GE Brown*, PJ Erwin, G Lavery', MI Halliday and KR Gardiner

Departments of Surgery, Queen's University of Belfast, Belfast, UK; *Beth Israel Deaconess Medical Center, Boston, USA; †Intensive Care Unit, Royal Victoria Hospital, Belfast, UK

Aims: Neurosurgical patients are at risk of multi-organ dysfunction. It was hypothesised that those patients requiring ICU admission would exhibit evidence of a systemic inflammatory response.

Methods: Over a period of 3 weeks, all neurosurgical patients (elective and emergency) admitted to a regional ICU had blood taken on the 1st and 3rd morning after admission. Blood was also taken from controls. Neutrophil (PMN) respiratory burst activity was assayed over 17.3 min using a BioOrbit 1251 Luminometer to detect diluted $(1: 20)$ whole blood chemiluminescence $(C L)$ in the presence of luminol. Circulating and maximal CL were measured by stimulation with phorbol 1,2-myristate 1,3-acetate in the absence or presence of tumour necrosis factor alpha (TNF) respectively (Fig. 1). The peak signal for each was obtained and the maximal value dived by the circulating value. This ratio represents the capacity to increase circulating respiratory burst activity. Soluble p55 TNF receptor (anti-inflammatory marker) and interleukin 6 (IL6, pro-inflammatory marker) were measured. Clinical parameters were recorded.

Results: Median [interquartile range, IQR] APACHE II scores were 22 (17-26) and 24 (11-27) on days 1 and 3 respectively.

Figure

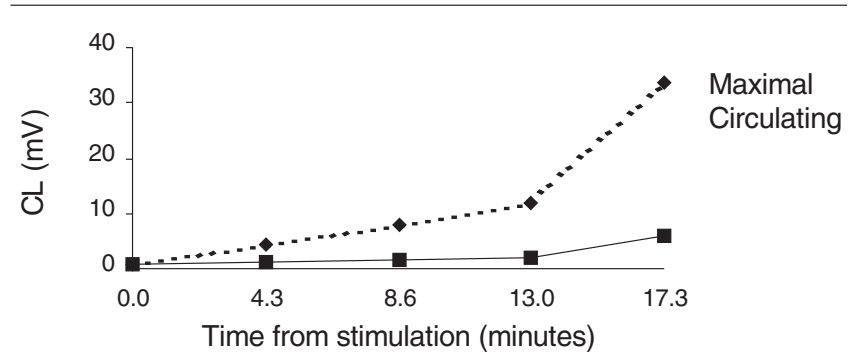

Example of chemiluminescent assay from one patient on day 3.

\begin{tabular}{lccc} 
& CL Ratio & p55 TNF $(\mathrm{ng} / \mathrm{ml})$ & IL6 $(\mathrm{pg} / \mathrm{ml})$ \\
\hline Day 1 $(\mathrm{n}=15)$ & $2.2[1.4-2.4]$ & $7.7[6.6-10.7]$ & $270.1[81.5-388.1]^{*}$ \\
Day 3 $(\mathrm{n}=7)$ & $4.0[1.5-4.0]^{+}$ & $9.0[6.3-12.8]$ & $216.9[36.0-294.0]^{\dagger}$ \\
Control $(\mathrm{n}=24)$ & $2.2[1.5-2.2]$ & $7.8[5.0-12.6]$ & $11.0[11.0-11.0]$ \\
\hline
\end{tabular}

Analysis by Mann-Whitney U. ${ }^{*} P=0.002$, day 1 vs control; ${ }^{\dagger} P<0.05$, day 3 vs control.

Conclusion: Patients admitted to ICU with neurosurgical insults have raised levels of pro-inflammatory cytokines with an increased capacity for PMN activation. Assays of PMN activation can be used as a measure of the balance between pro- and anti-inflammatory mediators.

\section{P183 Bacterial versus viral meningitis: comparison of the old and the new clinical prediction models}

\section{Y Tokuda}

Department of Medicine, Okinawa Chubu Hospital, Gushikawa city, Okinawa, Japan

Introduction: Accurate initial diagnosis is the cornerstone for therapeutic decision making of acute bacterial meningitis (ABM). A previously reported statistical model based on a combination of four parameters (total polymorphonuclear cell count in cerebrospinal fluid (CSF), CSF/blood glucose ratio, age and month of onset) appeared effective in differentiating acute viral meningitis (AVM) from acute bacterial meningitis in western countries. The objectives of this study were to validate this model on a independent sample of patients with acute meningitis seen in Okinawa, a tropical region of Japan, and to build a new model based on this sample. 
Methods: Retrospective review was performed for medical records of all persons aged more than 15 years for the management of community-acquired acute meningitis treated at a our hospital between 1985 and 1998. The criterion standard for bacterial meningitis was a positive CSF or blood culture. For viral meningitis, it was a discharge diagnosis of viral meningitis with no other etiology evident. A new prediction model was developed by using stepwise logistic regression analysis.

Results: Forty-five cases of bacterial meningitis and 101 cases of viral meningitis were confirmed. The discriminatory power of the old model as measured by the area under the receiver operating characteristic curve (AUC) was 0.695 (95\% Confidence Interval or Cl 0.545-0.845). The best representative model selected four slightly different independent variables: age, mental confusion, neck stiffness, and total CSF polymorphonuclear cell count. The area under the ROC curve was $0.986(95 \% \mathrm{Cl}$ $0.949-1.0)$, and significantly better than the old $(P<0.05)$.

Conclusion: In differential diagnosis of acute bacterial and viral meningitis, the old prediction model may not be clinically useful when applied to a geographically distinct population in a different ethnicity. Older age, mental confusion, neck stiffness, and higher CSF polymorphonuclear cell count were identified as the most accurate logistic model predicting the likelihood of bacterial meningitis on our patients.

\section{References:}

Spanos A, Harrel FE, Durack DT: Differential diagnosis of acute meningitis. JAMA 1989, 262:2700-2707.

McKinney WP, Heudebert GR, Harper SA, Young MJ, McIntire DD: Validation of a clinical prediction rule for the differential diagnosis of acute meningitis. J Gen Intern Med 1994, 9(1):8-12.

\section{P184 Protection of spinal cord ischemic injury with the $\beta$-agonist Clenbuterol}

\section{DaSilva and BY Lee}

New York Medical College, WCMC, Valhalla, NY, USA

We studied Clenbuterol, a selective $\beta-2$ agonist, in preventing ischemic injury to the spinal cord.

Methods: We used 30 New Zealand rabbits for their unique segmental arterial supply to the spinal cord. Fifteen animals in the study arm were given Clenbuterol $9 \mathrm{mg}$ PO $24 \mathrm{~h}$ before clamping. Fifteen (control arm) received water only. Heparin $(70 \mathrm{lU} / \mathrm{kg}$ ) was given $5 \mathrm{~min}$ before the incision. Ischemic injury was reproduced by cross-clamping the infrarenal aorta for either 22 or 30 min. Direct aortic blood flow measurements were taken with the transonic flowmeter before and after cross-clamping. Paralysis was assessed by the Tarlov's neurological scale.

Results: All animals ( 2 control, 2 study arm; $n=4$ ) subjected to a clamping time of $30 \mathrm{~min}$ developed complete paraplegia. Of the control group with 22-min clamping time $77 \%$ developed paraplegia (Tarlov $0, n=9$; Tarlov 1 , $n=1$ ) and $23 \%$ did not (Tarlov 3, $n=1$; Tarlov 4, n=2). In the experimental (Clenbuterol) group with 22-min clamping time $38 \%$ developed paraplegia (Tarlov $0, n=3$; Tarlov $1, n=2)$ and $62 \%$ did not (Tarlov $3, n=2 ;$ Tarlov $4, n=6)$. A consistent observation was that animals which developed paraplegia had a marked increase in post-declamping
Figure
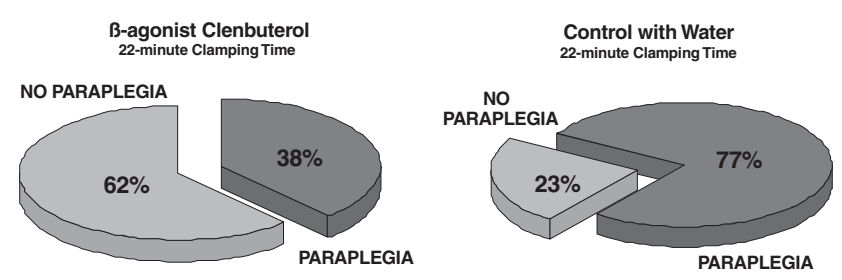

blood flow, i.e., hyperperfusion, suggesting that variations in aortic blood flow post-declamping may play a role in the development of paraplegia.

Conclusion: We concluded that Clenbuterol had a protective effect on the spinal cord in 62\% (experimental group) vs $23 \%$ (control) in animals subjected to a clamping time of $22 \mathrm{~min}$. Furthermore, controlling variations in aortic blood flow post-declamping may help to prevent paraplegia.

\section{P185 Therapy of malignant intracranial hypertension by lumbar cerebrospinal fluid drainage}

\section{E Münch*, P Vajkoczy', C Bauhuf ${ }^{\dagger}$, M Quintel* and P Schmiedek ${ }^{\dagger}$ \\ ${ }^{*}$ Departments of Anaesthesiology and Intensive Care Medicine and ${ }^{+}$Neurosurgery, Faculty of Clinical Medicine Mannheim, University of Heidelberg, Mannheim, Germany}

Objectives: The use of lumbar drainage in uncontrollable intracranial hypertension is considered to be contraindicated due to the possibility of inducing transtentorial or tonsillar herniation. The objective of this study was to investigate the effect of controlled lumbar CSF drainage on ICP and to evaluate the potential side effects of this 
new therapeutic modality in adult patients with therapyrefractory intracranial hypertension.

Methods: Twenty-three patients (age 36.09 \pm 17.4 years) with severe traumatic brain injury (TBI) $(n=12)$ or vasospasm after subarachnoid haemorrhage (SAH) $(n=11)$ were included prospectively. Patients were considered for lumbar CSF drainage if they suffered persistent intracranial hypertension $(>25 \mathrm{mmHg})$ for a period of more than $15 \mathrm{~min}$ and failed to respond to high intensity treatment. Lumbar CSF drainage was not performed in patients with tight basal cisterns. After institution of the lumbar drain, 5-20 ml CSF were initially aspirated and then continuous CSF drainage was maintained. ICP and CPP before and after bolus-aspiration were documented. The neurological outcome of the patients was scored according to the Glasgow Outcome Scale (GOS) 6 months after injury.
Results: Lumbar drainage was installed 4.8 43 days after $\mathrm{TBI} / \mathrm{SAH}$ and the average period of drainage was $6.6 \pm 5.1$ days. All patients showed an immediate and lasting decrease of ICP $(-17.4 \pm 11.4 \mathrm{mmHg} ; P<0.0001)$ and an increase of CPP $(+14.3 \pm 10.9 \mathrm{mmHg})$. Ten patients (44\%) showed a favourable outcome (good recovery or moderate disability), 4 patients (17\%) survived with a severe permanent neurological deficit, 1 patient (4\%) remained in a persistent vegetative state and 8 patients (35\%) died.

Conclusions: Controlled lumbar CSF drainage reduces therapy-resistant intracranial hypertension significantly. The hazard of transtentorial or tonsillar herniation might be limited by considering lumbar drainage only in the presence of discernible basilar cisterns.

\section{P186 Central neuroaxial blockade improves case-mix adjusted mortality of the critically ill surgical} patient

\section{AJ Prabhu, KR Burchett and MC Blunt}

Queen Elizabeth Hospital, Gayton Road, King's Lynn, Norfolk, PE30 4ET, UK

Introduction: Central neuroaxial blockade is known to provide good postoperative analgesia, hastens the return of gut function and may attenuate the 'stress response' to surgery [1]. However, there may be haemodynamic instability in critically ill patients. Despite improvements in many physiological parameters, there is little published evidence of improvement in outcome following surgery with such a block. Comparison of mortality outcome in intensive care units must take into account differences in the case-mix of patients in order to be meaningful. We investigated the effect of neuroaxial blockade on mortality standardised for case-mix using the APACHE 2 prognostic indicator (SMR) [2].

Methods: 205 adult patients admitted to the ICU for $>8 \mathrm{~h}$ following major abdominal surgery were categorised according to whether they had received neuroaxial blockade (block: 91 ; no-block 113) in a retrospective contemporaneous cohort-controlled design. APACHE 2 scores, calculated risk of death, types of surgery, ICU lengths of stay and hospital length of stay were collected from the ICU database, and SMRs were calculated for each group. Demographic data was assessed using ANOVA and SMR by Poisson distribution.

Results: There was a significant improvement in SMR in the block group $(P<0.01)$. The APACHE 2 scores and calculated risks of death were significantly lower in this group. Both hospital and ICU length of stay were significantly shorter in the block group. There were significant differences in the type of surgery performed between the two groups (Table).
Table

\begin{tabular}{lcc}
\hline & Block & No Block \\
\hline APACHE 2 & $15.0(13.9-16.0)^{\star \star}$ & $16.7(15.5-17.9)$ \\
Risk of Death (\%) & $19.1(16.1-22.0)^{\star \star \star}$ & $27.6(23.8-31.6)$ \\
ICU length of stay (days) & $1.3(0.4-13.9)^{\star \star}$ & $1.9(0.4-28.7)$ \\
$\begin{array}{l}\text { Time to hospital } \\
\text { discharge (days) }\end{array}$ & $12.5(1-107)^{\star}$ & $17(1-95)$ \\
$\begin{array}{l}\text { Standardised Mortality } \\
\text { Ratio (95\% Cl) }\end{array}$ & $0.46(0.23-0.92)^{\star \star}$ & $1.41(1.05-1.90)$ \\
\hline
\end{tabular}

Data presented as mean (95\% Confidence intervals) for age, APACHE and risk of death; median (range) for time data. ${ }^{\star} P<0.05$; ${ }^{\star \star} P<0.01$, ${ }^{\star \star \star} P<0.001$.

Conclusions: Within the methodological restrictions of the non-randomised design this study demonstrated an improvement in outcome in critically ill patients who receive neuroaxial blockade. The use of SMR as the primary end-point ensures a meaningful comparison of the groups despite the obvious difference in severity of illness between the groups as it allows outcome comparison for critically ill patients against a previously defined standard.

\section{References:}

1. Buggy DJ, Smith G: BMJ 1999, 319:530-531.

2. Rowan KM, Kerr JH, Major E, McPherson K, Short A, Vessey MP: BMJ 1993, 307:972-977. 


\section{P187 Alteration of current perception threshold in severely injured patients}

M Miyake, N Hitosugi, K Ikegami, H Inoue, Y Sato and I Sato*

Trauma and Critical Care Center, Koshigaya Hospital, Dokkyo University School of Medicine; *Dept of Anesthesiology, 2-1-50

Minamikoshigaya Koshigaya-shi, Saitama, Japan 343-8555

Background: Measurements and assessment of current perception threshold with Neurometer in patients with chronic pain is well established. However, pathogenesis of acute pain in severely injured patients is not fully understood.

Purposes: To measure current perception threshold in severely injured patients with and without intravenous fentanyl and to determine if pain threshold is increased with sufficient analgesia.

Patients and methods: Twenty severely injured patients with normal mental status were studied. Patients were given bolus injection of fentanyl $(1 \mathrm{~g} / \mathrm{kg})$ upon arrival at the emergency room. After resuscitation and surgery, patients were transferred to ICU and given continuous intravenous fentanyl started at $50 \mathrm{~g} / \mathrm{h}$ and increased up to $150 \mathrm{~g} / \mathrm{h}$ on patient's demand. Pain threshold (PT) was measured with a Neurometer. VAS (visual analogue scale) and VRS (verbal rating scale) were also measured before and after bolus injection of fentanyl and during ICU care (every hospital day until seventh).

Findings: Before bolus injection of fentanyl, PT of the patients was significantly lower than that of control (106 $\mathrm{mA}$ vs $221 \mathrm{~mA} ; P<0.01)$. After continuous intravenous fentanyl in ICU, VAS decreased significantly. However, no change was observed in PT of the patients during continuous analgesia with fentanyl.

Conclusions: Severely injured patients were more sensitive to nociceptive stimuli than normal healthy controls. Fentanyl is an effective analgesia in trauma patients and decreased VAS significantly in the patients. But pain threshold itself did not show any change even with sufficient analgesia.

\section{P188 The early management of pain in casualty wards}

\section{F Van Trimpont and M Genard}

Ambroise Pare University Hospital, Mons, Belgium

Objectives: Pain is often the main complaint of patients coming into casualty wards but its management is rarely initiated early. Pain management must be considered as an aspect of the general management of the patient coming into the emergency ward $[1,3]$. It is consequently essential to relieve the pain quickly while continuing the diagnosis [2]. The treatment of pain is a progressive process and there should be no hesitation in combining different drugs with a maximum analgesic benefit while controlling the analgesia in order to avoid secondary effects. The following protocol will allow rapid and reassuring analgesia.

Method: Upon arrival in the ward, the adult patient is dealt with by a nurse who evaluates the analgesic need using the Visual Analogue Scale (VAS). After the consent of the doctor by signing a standard protocol, proparacetamol (2 $\mathrm{g}$ IV) and diclofenac (75 mg IV) are administered every 6 and $12 \mathrm{~h}$ respectively, irrespective of the VAS level. If the VAS is greater than 3 in the half hour following the initial administration of proparacetamol and diclofenac, the analgesia is completed by subcutaneous morphine (the dose is defined on the written protocol in relation to the weight and age of the patient). This administration must be repeated with an interval of $4 \mathrm{~h}$ if the VAS is greater than 3. The contraindications for the different drugs must be respected: chronic renal insufficiency, arterial hypertension with heart failure and gastric ulcers for diclofenac; hepatic insufficiency, atopy, nasal polyps, asthma and eczema for proparacetamol; chronic respiratory insufficiency and drug addiction for morphine.

Results: The cases collected $(n=200)$ show a demographic equivalence ( $45 \%$ women, $55 \%$ men) and an average age of 49 years. The distribution of the cases was mainly orthopedic $(72 \%)$, with renal colic representing $9.6 \%$. Other less common indications made up the remaining 18.4\% (neuralgia, arthritis, knife wounds, gout, colitis). The Table shows the VAS over time, from admission up to $28 \mathrm{~h}$ (for 63 patients).

Table

\begin{tabular}{lcccc}
\hline & Average & Standard deviation & Quantity & $\%$ VAS $>3$ \\
\hline Hour O $(\mathrm{H} 0)$ & 6.02 & 2.06 & 73 & 88 \\
$\mathrm{H} 0+4$ & 3.6 & 1.07 & 73 & 45 \\
$\mathrm{H} 0+8$ & 3.64 & 1.09 & 51 & 38 \\
$\mathrm{H} 0+12$ & 3.55 & 1.05 & 48 & 32 \\
$\mathrm{H} 0+16$ & 2.99 & 0.94 & 39 & 13 \\
$\mathrm{H} 0+20$ & 2.97 & 1.05 & 31 & 13 \\
$\mathrm{H} 0+24$ & 2.78 & 0.93 & 30 & 8 \\
$\mathrm{H} 0+28$ & 2.48 & 0.85 & 20 & 4 \\
\hline
\end{tabular}


The average VAS on admission was 6.02 \pm 2.06 .

Conclusions: Using a simple standardized method this protocol allows effective and early management of pain with combined analgesia in a casualty ward.
References:

1. Berthier F, Potel G, Le Conte P, Tonze MD, Baron D: Comparative study of methods of measuring acute pain intensity in an emergency department. AM J Emerg Med 1998, 16:132-136.

2. Berthier F, Le Conte P, Garrei P, Potel G, Baron D: Analyse de la prise en charge de la douleur aiguë dans un service d'accueil et d'urgence. Réan Urg 1998, 7:281-285.

3. Blettery B, Bhrahim L, Honart D, Aube H: Les échelles de mesure de la douleur dans un service d'accueil des urgences. Réan Urg 1996, 16:691-697.

\section{P189 Monitoring of sedation with BIS Index, comparison with Ramsay and Cook sedation scores}

\section{R Šplechtna, L Pokorný, E Hušková and D Nalos}

ICU, Masaryk Hospital, Pasteurova 9, 40113 Ústí nad Labem, Czech Republik

Introduction: Sedation and analgosedation are an integral part of intensive care medicine. The level of sedation is often very important for the final result of the treatment. Different sedation scores are used to asses this level (Ramsey, Cook, etc.). But these scores can be very subjective, dependent on a observer and can show quite great interindividual differences. The Bispectral index (BIS) is a processed EEG parameter and shows continuously the level of inhibition of brain function during application of drugs for anesthesia and sedation.

We have tried to find if there is any relation between the value of the BIS index and the Ramsay or Cook score. In the case of a positive result, there can be a chance of exact titration of drugs and possible economical profit.

Methods: The group of 20 patients hospitalized in our ICU (October 1998-September 1999). Including criteria-GCS 15 and the need of continual sedation or analgosedation.

Figure 1

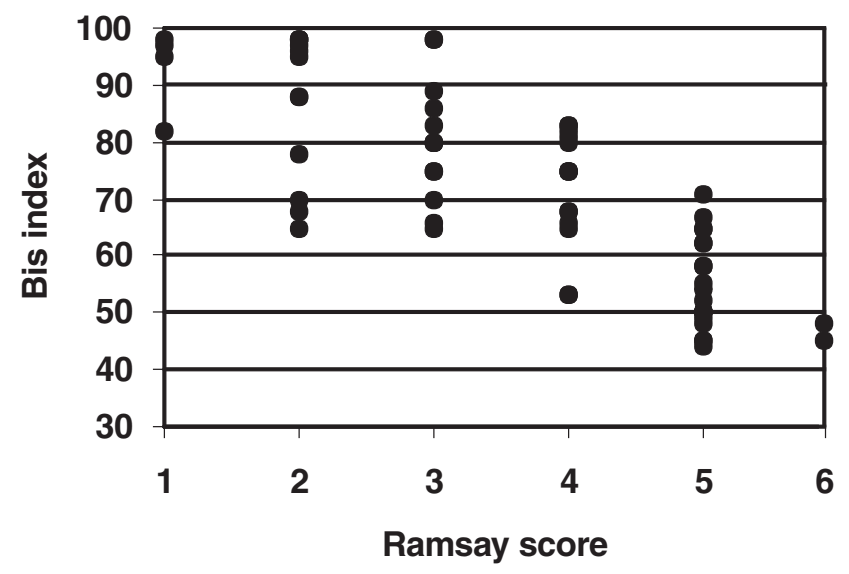

Figure 2
Monitoring of all patients by the monitor 'Aspect A-1000 TM'. The values of the BIS index were recorded to protocol at any time of change in the level of sedation. At the same time this level of sedation was evaluated by two physicians (to prevent interindividual differences) by the use of Ramsay and Cook scores. All 75 examinations were recorded to study protocol. Results were assessed by help of means (median) + SD (range) and graphs.

Results: See Figs 1 and 2.

Conclusion: Figures 1 and 2 demonstrate that a narrow span of BIS index values includes a very wide scale of a depth of sedation and analgosedation assessed by Ramsay and Cook scores. These results and especially our experiences with continual monitoring show that to rely only on BIS index or to titrate drugs according to these values is impossible. We admit that bigger number of patients is needed for better evaluation.

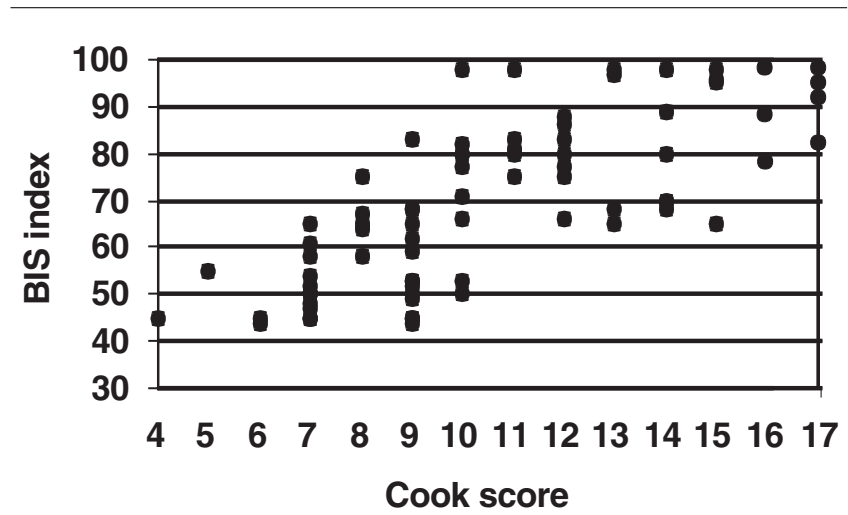




\section{P190 Bispectral index (BIS) monitoring of ICU patients on continuous infusion of sedatives and paralytics reduces sedative drug utilization and cost}

\section{LJ Kaplan and H Bailey}

Medical College of PA Hospital, Departments of Surgery and Emergency Medicine, Division of Trauma and Critical Care, 3300 Henry Avenue, Philadelphia, PA 19129, USA

Purpose: To determine whether BIS-monitored titration of sedatives in ICU patients on continuous infusions of sedatives and paralytics was cost effective and reduced the incidence of the recall phenomenon.

Methods: Four consecutive months of patients in a SICU on continuous infusions of paralytics and sedatives were studied. Patients with severe brain injury (GCS $\leq 8$ ) were excluded. In months one and two, sedatives were titrated to patient comfort guided by vital sign changes after stimulation. Months three and four titrated sedative infusions to a BIS value between 70 and 80 . Paralytic agents were titrated to a train-of-four response $(2 / 4$ at $50 \mathrm{~mA})$ on a protocol. Demographic data, drug usage, as well as drug cost were abstracted. On ICU discharge, patients were queried about recalling pain or fear while they were on continuous infusions of paralytics and sedatives. Data are means \pm standard deviations and were compared by unpaired t-test or $\chi^{2}$ as appropriate; significance assumed for $P<0.05$.

Results: During months one and two, the cost per patient $(n=31)$ for sedatives was $\$ 819 \pm 2.045$. Patient diagnosis was $66 \%$ trauma, 22\% abdominal sepsis, 9\% vascular, and $5 \%$ other. The most frequently used medications and their percent contribution to total costs were morphine $58 \%$ (5.8\% cost), lorazepam 54.8\% (63.2\% cost), midazolam $12.9 \%$ (4.2\% cost), and propofol $12.9 \%(24.6 \%$ cost). $18 \%$ of the patients recalled a frightening or painful experience during their period of continuous sedation. During months three and four, the cost per patient $(n=26)$ for sedatives was $\$ 669 \pm 1.362$ - an $18 \%$ reduction in cost versus months one and two $(P>0.05)$. Only $4 \%$ of patients recalled frightening or painful events $(P<0.05)$. Demographics were similar compared to months one and two $(P>0.05)$. Significant reductions occurred in lorazepam $(18 \%)$, midazolam (18\%) and propofol (47\%) usage $(P<0.05) .15 .4 \%$ of patients who appeared sedated were under-sedated by BIS analysis, while $54 \%$ of patients required less sedative than initially predicted by the staff.

Conclusion: Bispectral index monitoring of ICU patients on continuous infusions of paralytics and sedatives reduces sedative drug cost as well as the recall phenomenon. Significant under-sedation may occur using subjective analysis of sedation in the ICU.

\section{P191 The change in Bispectral Index with stimulation indicates depth of sedation in intensive care}

AT Dewhurst, S Chieveley-Williams and J Goldstone

Centre for Anaesthesia, UCL Hospital, London W1N 8AA, UK

Introduction: Bispectral Index (BIS) is an EEG parameter which gives a continuous reading between 0-100 depending on depth of anaesthesia. It has been used in ICU as a monitor of sedation [1]. Clinical assessment of sedation requires the patient to be stimulated, which may cause elevation of the BIS. We proposed that this change in BIS in response to stimulation is important when assessing depth of sedation. The aim of this study was to assess the feasibility of BIS as a monitor of sedation in ICU and to quantify within different sedation groups the changes in BIS score before and after stimulation.

Method: ICU patients being ventilated and sedated were studied. BIS was recorded on an Aspect A2000 monitor. $A$ nurse blinded to the BIS reading assessed sedation using the observer's assessment of alertness/sedation score (OAAS 1-5: 1=no response, 5=fully awake). Sedation was assessed hourly, average unstimulated BIS (BISu) measured before clinical assessment of sedation was compared to average stimulated BIS (BISs). Spearman's correlation coefficient was used to assess association between BIS and OAAS, Mann-Whitney $U$ was used to compare differences between BIS in OAAS groups 4-5 (lightly sedated), OAAS groups 1-3 (deeply sedated)

Figure

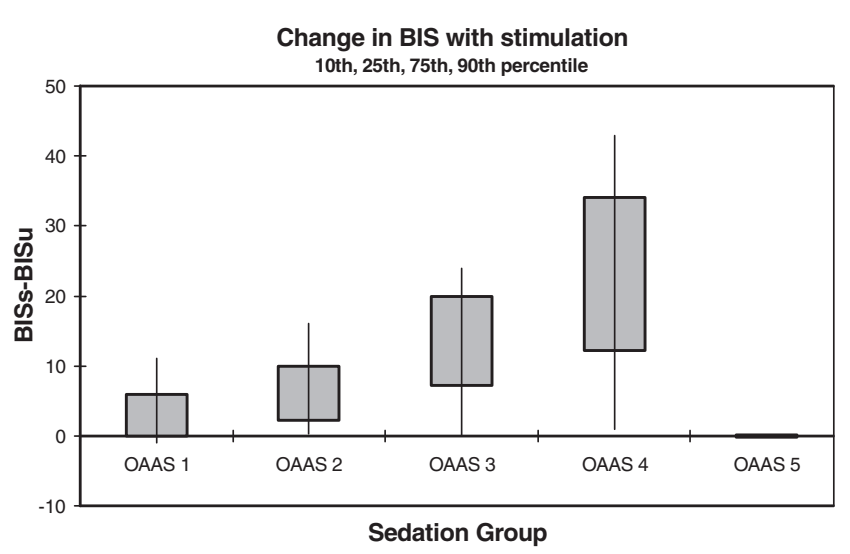


and in the change of BIS ( $\triangle \mathrm{BIS})$ with stimulation in all OAAS groups.

Results: A total of 139 observations in 30 patients were recorded. There was a significant difference $(P<0.05)$ between BIS score in the lightly sedated group and deeply sedated group. Correlation coefficient BISu/OASS $r=0.53$, BISs/OASS $r=0.73$. $\triangle$ BIS was larger in OAAS 3-4 than in OAAS 1,2 and 5.
Conclusion: BIS can distinguish between lightly and deeply sedated patients. BISs correlated more closely with OAAS than BISu. On stimulation $\triangle \mathrm{BIS}$ was greatest in OAAS 3-4. BIS can be used in ICU patients but readings should be interpreted over time and in response to stimulation.

\section{Reference:}

1. Simmons L et al.: Critical Care Medicine 1999, 27:1499-1504.

\section{P192 Dexmedetomidine for sedation in the medical ICU}

\section{RM Venn, J Ball, A Steele, PJ Newman and RM Grounds}

St George's Hospital, Blackshaw Road, London SW17 OQT, England

Introduction: The highly selective alpha-2 agonist, dexmedetomidine, has been shown to be an effective and safe agent for the sedation of postoperative patients requiring mechanical ventilation on the ICU [1]. It possesses analgesic and sympatholytic properties and its ability to sedate without significant respiratory depression may benefit other patient groups requiring ICU sedation. This study reports our experience of dexmedetomidine for the sedation of critically ill medical patients.

Methods: Twelve critically ill medical patients (mean APACHE II score 21) requiring artificial ventilation, received dexmedetomidine for up to seven days with rescue sedation and analgesia provided by propofol and morphine, respectively, if clinically indicated. Dexmedetomidine infusion was commenced with a loading infusion of $1 \mu \mathrm{g} . \mathrm{kg}^{-1}$ over $10 \mathrm{~min}$ followed by a maintenance infusion of $0.2-0.7 \mu \mathrm{g} / \mathrm{kg} / \mathrm{h}$ to maintain a Ramsay sedation score $\geq 3$. Following experience with the first four patients, the maintenance rate of infusion could be increased to a maximum of $2.5 \mu \mathrm{g} \cdot \mathrm{kg}^{-1} \mathrm{~h}^{-1}$. Heart rate, systolic and diastolic arterial pressures, central venous pressure and, where possible, cardiac output by thermodilution were monitored continuously and recorded at $10 \mathrm{~min}$ intervals for the first $30 \mathrm{~min}$ and then hourly.
Results: The mean duration of dexmedetomidine infusion for all patients was $33(S D=19.7) h$, and mean dexmedetomidine infusion rate in the latter eight patients was $1.0(\mathrm{SD}=0.7) \mu \mathrm{g} \cdot \mathrm{kg}^{-1} \mathrm{~h}^{-1}$. Only one out of the eight patients proved difficult to sedate with dexmedetomidine and required an additional propofol infusion of up to $100 \mathrm{mg} \cdot \mathrm{h}^{-1}$. Arterial pressure, heart rate and cardiac output were well-maintained during dexmedetomidine infusion. Patients requiring vasoactive drugs prior to commencement of dexmedetomidine showed diminishing requirements during the course of the study. Following discontinuation of dexmedetomidine infusion there was no clinically important rebound phenomenon in haemodynamic measurements. Adverse cardiovascular events (hypotension \pm bradycardia) were nearly all confined to the initial loading infusion of dexmedetomidine.

Summary: Dexmedetomidine provides safe, titratable sedation with cardiovascular stability for critically ill medical patients requiring artificial ventilation.

\section{Reference:}

1. Bradshaw CJ, Venn RM, Spencer R: Cardiovascular effects of dexmedetomidine for ITU sedation: UK results of a multi-centre study. Critical Care 1999, 3:P233.

\section{P193 Respiratory effects of dexmedetomidine in the ICU}

J Hell, RM Venn, R Cusack, A Rhodes and RM Grounds

St George's Hospital, Blackshaw Road, London SW17 OQT, England

Introduction: The incidence of haemodynamic disturbance and myocardial ischaemia in the ICU can be reduced if sedation is continued over the peri-extubation period [1]. Dexmedetomidine has been shown to cause only minimal ventilatory depression in human volunteers [2] and also possesses analgesic and sympatholytic properties and so may be a useful agent for this peri-extubation period.

Methods: Twenty-five patients involved in a randomised, placebo-controlled, double-blind study were ventilated for at least $6 \mathrm{~h}$ postoperatively and were sedated with dexmedetomidine or placebo infusions. They were then extubated whilst receiving these respective infusions. Following extubation, infusions (Dexmedetomidine 0.2-0.7 $\mu \mathrm{g} / \mathrm{kg} / \mathrm{h}$ ) were continued for six hours to maintain a Ramsay sedation score $\geq 2$. Rescue sedation and analgesia was provided by midazolam and morphine respectively, if clinically required. Arterial blood gas estimation, oxygen saturation, respiratory rate and analgesic requirements were recorded for the peri-extubation period. Results are reported as mean (SD) and analysed using MannWhitney-U and ANOVA for repeated measures where appropriate. 


\begin{tabular}{|c|c|c|c|c|c|c|c|}
\hline & \multicolumn{2}{|c|}{2 h post-extubation } & \multicolumn{2}{|c|}{$4 \mathrm{~h}$ post-extubation } & \multicolumn{2}{|c|}{$6 \mathrm{~h}$ post-extubation } & \multirow[b]{2}{*}{$P$ value } \\
\hline & Dexmed. & Placebo & Dexmed. & Placebo & Dexmed. & Placebo & \\
\hline Resp. rate & $18.0(4.6)$ & $20.4(6.1)$ & $16.6(1.5)$ & $20.1(5.2)$ & $16.8(2.8)$ & $19.5(4.5)$ & 0.05 \\
\hline $\mathrm{PaCO}_{2}$ & $5.4(0.7)$ & $5.5(0.9)$ & $5.8(1.0)$ & $5.6(0.6)$ & $5.4(0.6)$ & $5.3(0.8)$ & NS \\
\hline $\mathrm{pH}$ & $7.4(0.1)$ & $7.3(0.1)$ & $7.3(0.1)$ & $7.3(0.1)$ & $7.4(0.1)$ & $7.4(0.1)$ & NS \\
\hline $\mathrm{PaO}_{2} / \mathrm{FiO}_{2}$ & $38.4(16.5)$ & $30.0(10.3)$ & 39.5(13.3) & $29.2(7.2)$ & $43.0(13.2)$ & $29.0(8.0)$ & 0.02 \\
\hline
\end{tabular}

Results: Patient demographics and degree of sedation and analgesia was equivalent in the two groups but the placebo group required three times more morphine $(P=0.04)$. The average dexmedetomidine infusion rate was $0.15 \mu \mathrm{g} \cdot \mathrm{kg}^{-1} \mathrm{~h}^{-1}$ following extubation. No adverse respiratory events were seen in either group.
Summary: Dexmedetomidine is safe to use in extubated spontaneous breathing intensive care patients and does not cause clinically significant respiratory depression.

\section{References:}

1. Br J Anaesth 1998, 80(6):834-836.

2. Anesthesiology 1992, 77(6):1125-1133.

\section{P194 Lactic acid clearance in the emergency department prognosticates multisystem organ failure and death}

BP Knoblich, EP Rivers, HB Nguyen, MT Mullen, B Rittinger, G Hays, A. Muzzin, B Sheridan, M Jankowski and MC Tomlonovich

Departments of Emergency Medicine, Internal Medicine and Surgery, Henry Ford Hospital/Case Western Reserve University, 2799 West Grand Blvd. Detroit, Michigan 48202 USA

Background: Lactic acid (LA) indicates anaerobic metabolism (global tissue hypoxia). While single LA levels are helpful diagnostically and therapeutically; the change in LA levels over time (LA-clearance) has better prognostic value in predicting morbidity or multisystem organ failure (MSOF) and death in the intensive care unit (ICU).

Objective: To examine the relationship of LA-clearance in the Emergency Department (ED) and the development of multisystem organ failure and death.

Methods: This was a prospective case series of critically ill patients presenting to a large urban ED in shock (systolic blood pressure $<90 \mathrm{mmHg}$ for thirty min after a $40 \mathrm{cc} / \mathrm{kg}$ volume challenge) or a LA $>2 \mathrm{mM} / \mathrm{l}$ on arrival. Patients presenting in hemorrhagic shock, trauma, requiring immediate surgery, do not resuscitate orders, seizures or end-stage disease not benefiting from ICU care were excluded. Patients were managed by ED physicians by standard ED management. LA and Multisystem Organ Dysfunction Scores (MODS) were obtained on ED arrival, discharge and every $12 \mathrm{~h}$ for $72 \mathrm{~h}$. Patients were grouped by the lactic acid clearance during the ED stay: Group 1 (no clearance, $<0 \mathrm{mM} / \mathrm{l} / \mathrm{h}$, actual increase in lactic acid), Group 2 (intermediate clearance, $>0$ and $<1 \mathrm{mM} / \mathrm{l} / \mathrm{h}$ ), and Group 3 (high clearance, $>1 \mathrm{mM} / / / \mathrm{h}$ ). Student's T-test and ANOVA were used to compare the MODS score and LA over time, with Bonferroni correction for multiple comparisons.

Results: A total of 142 patients were studied. The mean age was $65.9 \pm 17.2$ years. The lactic acid clearances for groups 1,2 and 3 were $-0.45 \pm 0.55,0.48 \pm 0.48$, $1.44 \pm 0.38 \mathrm{mM} / \mathrm{l} / \mathrm{h}$ respectively $P<0.0001$. The mean MODS scores over $72 \mathrm{~h}$ were $8.63 \pm 3.35,6.16 \pm 4.23$, $5.12 \pm 3.76$ for groups 1,2 and 3 respectively $P<0.02$. The in-hospital mortality was $50 \%, 23 \%, 12 \%$ in groups 1 , 2 and 3 respectively $P<0.045$.

Conclusions: The duration of global tissue hypoxia or decreased LA clearance in the ED is associated with MSOF and death. This pathogenic link suggests that diagnostic and therapeutic intervention should begin immediately in the ED.

\section{P195 Earthquake, rescued victims, crush injury and their follow up}

\section{N Ünal, M Oral, AA Yilmaz and M Tulunay}

Department of Anaesthesiology and Reanimation, University of Ankara, Faculty of Medicine, Ankara, Turkey

Thirty complicated crush injury patients were accepted to our ICU after the Big Marmara (Turkey) earthquake in August 1999. Results of 18 patients were reviewed and are presented below.
The mean ages of 10 male and 8 female patients were $31.1 \pm 12.6$ (range: $15-55$ ) years. Time from earthquake to salvage and first medical aid were 12.5 \pm 9.4 (range: 4-40) and 17.5 \pm 10.9 (range: 6-41) h, respectively. 
Victims were accepted into our unit $5 \pm 8.4$ (range: $1-35$ ) days after the disaster. Their initial problems were acute renal failure (13), respiratory failure (5), disseminated intravascular coagulopathy (DIC) (4), bleeding (3), long bone fractures (2), pelvic fracture (2), lung contusion (1), haemodynamic instability (1), sepsis (1), in addition to crush injuries in the lower extremities (14) and upper extremities (6). Decompressive fasciatomies had been performed for all patients with crush injuries before acceptance to our ICU. Patients were treated for acute renal failure (14); DIC (13); sepsis (13) [originating from wounds (11), urinary system (9) and respiratory system (7)], respiratory failure (10), hepatic dysfunction (7) and cardiac dysfunction (3) during their stay in the ICU. Mean dialysis requirements for patients with renal failure were $5.1 \pm 3.6$ (range: $3-13$ ) for a period of $10.1 \pm 6.8$ (range: 6-23) days. Venovenous hemofiltration was performed twice for two patients and once for four patients because of volume overload. Fresh frozen plasma, 9.5 \pm 10.4 (range: 0-37) units; platelet suspension, 5.2 \pm 7.1 (range: 0-21) units; cryoprecipitate, $3 \pm 6.6$ (range: 0-26) units; and erythrocyte suspension, 18.6 \pm 10.6 (range: 6-39) units were transfused because of preDIC/DIC and massive bleeding especially after excision of debris. Antithrombin III replacement was performed in 8 patients. Ten patients with respiratory failure were mechanically ventilated for $9 \pm 14.8$ (range: 3-55) days. Amputations were required in six patients.

Eventually two patients with MOF died and 16 patients were discharged to other clinics with some sequels like neuropathies (10) and cardiomyopathy (1). One patient was discharged for hyperbaric oxygen therapy because of deep soft tissue infection unresponsive to medical therapy.

\section{P196 Experience of medical examination and health consultation for public exposed to neutrons caused by criticality incident in Japan}

Y Haraguchi, Y Tomoyasu, T Satoh, K Neriishi, N Oohashi, M Hoshino and T Arai

National Hospital Tokyo Disaster Medical Center, Tokyo, Japan

A criticality incident occurred September 30th, in Tokaimura in Japan. Neutron radiation was emitted in a widespread area. In addition to three patients, who were exposed to high dose neutron radiation, people who were living around the incident plant (within $350 \mathrm{~m}$ ) were urged to take shelter according to an evacuation order, and people who were living within $10 \mathrm{~km}$, were also recommended to remain within their houses for three or four days.

We had an opportunity to examine and give advice to the people who feared the radiation exposure. The experience is reported.

Results: The criticality incident continued for nearly one day. From the next day after the end of criticality was declared, we started health examination. During the consecutive three days, a total of 1852 people were examined. The complete blood count, including the hemogram or lymphocyte counts, and blood and urine chemistry were evaluated in all the patients. The survey of radiation contamination was done when patients requested. The history of the health condition and estimation of the radiated amount was evaluated by our members (physicians) in all the patients. Although many people complained of the fear of the after effects of radiation, the degree of fear was extremely strong in children and pregnant women. Regarding the results of blood and urine examination, there were no patients who had abnormalities that were thought to be caused by high amounts of radiation exposure, although there were several patients who had decreased lymphocyte counts.

Discussion and conclusions: Consultation only seems to be justified because, so far as we are informed officially (from the Japanese Agency of Science and Technology), the amount of radiation exposure was within the safe range. However, several patients who had decreased lymphocyte counts are recommended to receive repeated examination of the blood counts. Mental care and long-term follow-up may be necessary for people who are fearing the exposure and for children/pregnant woman.

\section{P197 Establishment of emergency medical services in Addis Ababa, Ethiopia}

O Benin-Goren*, P Halpern*, S Amir ${ }^{\dagger}$ and MB Tesfay ${ }^{\ddagger}$

${ }^{*} E D$ Tel Aviv Sourasky Medical Center Israel; ${ }^{\dagger}$ Sapir Medical Center Israel; ${ }^{\ddagger}$ Armed Forces General Hospital, Addis Ababa Ethiopia

In February 1998, an Israeli Medical Delegation that included two ED Directors and one Deputy Head Nurse of the ED, was founded in order to introduce the emergency medicine (EM) profession and to help in establishing an EM system in Addis Ababa. The program was produced in coordination with an Ethiopian physician who visited the
ED in his fellowship and was very impressed with the Israeli system.

With the approval of the International Relation of the Foreign Bureau of the State of Israel, the team traveled to 
Ethiopia to consider the possibility that the program could be changed according to the needs in the field.

The program consisted of several issues in EM: administrative concepts of ED building; organization of the ED; the role of Emergency Nurses in the team; trauma and the treatment of critical care; study and practices of Basic Life Support; advanced Cardiac Life Support; and Trauma Life Support.

The participants on the program included twenty physicians and ten nurses who work in emergency services. The first meeting was dedicated to study the existing system in Addis Ababa. With poor conditions, lack of necessary equipment, medication and resources, and with lack of basic knowledge, the health system in Addis Ababa cannot start re-organization without help.

Yet there is a new movement of physicians who are ready to take responsibility and be part of the change. The Israeli team gave the recommendation to the authorities in Israel to continue education among the Ethiopian staff-nurses, physicians, technicians, and Paramedics.

This paper examines EM in Addis Ababa, the course, and recommendation for the future. Furthermore, in addition to the program that has been developed until now, the Israeli team with the cooperation of some American physicians plans to continue the program in December 1999 that will include studies and meetings with policy makers in Addis Ababa.

\section{P198 A prospective trial of surgical resident performed focused abdominal sonography for trauma (FAST): an easy training method}

\section{DM Bridge, M Bonta, J Blackford, W Wiand, D Giammar and LM Foley}

Riverside Methodist Hospitals, 3535 Olentangy River Road, Columbus, Ohio 43214, USA

Introduction: Ultrasound is a quick, easy tool for evaluating trauma patients. A focused abdominal sonogram for trauma (FAST) is a limited sonographic evaluation easily learned by those with minimal experience. Our study attempts to prove that surgical residents of all postgraduate levels can be trained with a short didactic session followed by hands-on experience, studying patients with known intra-abdominal fluid. With this method, surgical residents can be quickly and cost-effectively taught to perform FAST examinations with proficiency rates matching those of the literature.

Materials and methods: In this prospective randomized trial, thirteen surgical residents were trained to perform FAST scans. Residents attended a 3-hour didactic course and then performed three normal FAST scans followed by four scans on patients with known intra-abdominal fluid. Lastly, trauma patients over a six-month period were studied. FAST results were compared to CT, DPL, or serial examination results. Statistical analysis was performed.
Results: In $93.8 \%$ of patients no fluid was detected. There was one false positive and one false negative exam. The sensitivity and specificity were $80 \%$ and $99 \%$, respectively. Positive and negative predictive values were $80 \%$ and $99 \%$, respectively. A kappa statistic of 0.79 was calculated indicating a substantial amount of agreement between the FAST scan group and the CT/DPL/serial exam group.

Discussion: Sonography guidelines for the training of surgical residents are needed. By maximizing positive examinations, the number of required proctored examinations can be lowered and the learning curve shortened. In a short time, surgical residents can learn to perform the FAST scan with proficiency rates matching that of the literature.

Conclusion: By training on patients with known intraabdominal fluid, in a short time, surgical residents can learn the FAST scan. With our method, resident proficiency can match that of more experienced personnel.

\section{P199 Data from the U.S. Army field surgical hospital intensive care unit in post-war Kosovo}

\section{SM Grosso}

FACS, Major, Medical Corps, U.S. Army

Objective:To review the admissions to the intensive care unit in a military field surgical hospital to determine the relationships, if any, between demographics, severity of illness, length of stay, ventilatory duration, and other parameters for operative and non-operative trauma, nontrauma surgical, and medical admissions.

Design: Prospective observational study.
Setting: U.S. Army 67th Combat Support Hospital intensive care unit deployed to a forward field location in the U.S. Sector in post-war Kosovo in support of the NATO peacekeeping mission in that region.

Patients: All fifty-five patients admitted to the intensive care unit from July through November, 1999 were enrolled in the study. 
Interventions: None.

Measurements and main results: Therapeutic Intervention Scoring System (TISS) Scores for each patient were determined at admission and at discharge. Other data collected included demographic data, including nationality, age, and gender; diagnosis; length of stay; duration of ventilatory support, if applicable; and disposition at discharge.

Conclusions: The majority (68\%) of patient admissions were for trauma. Patient age ranged from 1 day to 76 years. Sixty-two percent of patients were 42 years of age or younger. Mean length of stay was 1.70 days. Seventyone percent of patients were local national (Kosovo) patients, and 29\% were NATO personnel. Mean TISS scores for trauma, non-trauma surgical, and medical admissions were $21.67,17.00$, and 9.53 , respectively. There was a positive correlation between TISS score at admission and length of stay for all patient categories. Mean delta TISS scores between admission and discharge were $12.14,8.00$, and 5.00 for trauma, non-trauma surgical, and medical admissions respectively. Patients who remained on the ventilator for prolonged periods had higher TISS scores at admission (mean 36.71) than the population overall (mean 18.23). Length of stay was determined by many variables, including severity of illness, diagnosis, availability of host nation facilities for local patient transfers, and urgency of evacuation for military and other NATO personnel.

\section{P200 Management of children with severe traumatic brain injury in an adult ICU: evaluation of outcome}

G Tsaousi, A Lavrentieva, D Setzis, Ch Skourtis, E Anastasiou and M Yannakou

Department of Anaesthesiology and ICU, AHEPA University Hospital, St. Kiriakidi 1, 54006, Thessaloniki, Greece

Introduction: Injuries are the leading cause of death in children and brain injury is the most common cause of paediatric traumatic death. Shortage of paediatric intensive care units imposes children's occasional admission in an adult ICU. We attempted to evaluate the effectiveness of their management in a nonspecialised environment (adult ICU).

Methods: Data of 31 children with severe traumatic brain injury (STBI), admitted in our ICU, were reviewed retrospectively. Cases were analysed according to the following criteria: 1) age in years, 2) GCS (upon admission), 3) CT- scan grade, 4) injury severity score (ISS), 5) paediatric trauma score (PTS), 6) paediatric risk of mortality (PRISM III-24h), 7) length of ICU stay in days and 8) outcome at ICU discharge (Glasgow outcome score GOS). The demographic data of the studied group are shown in the Table.

Results: 1) Mean age was 6.4 years (SD \pm 5$)$. 2) Trafficrelated accidents were responsible for $67.7 \%(n=21)$ of cases, $29.1 \%(n=9)$ were attributed to fall from height and $3.2 \%(n=1)$ to gunshot. 3) The average length of ICU stay was 5.1 days. 4) Eighteen patients (58.1\%) fully recovered (GOS 5), 7 patients (22.5\%) presented moderate neurological dysfunction (GOS 3-4) and 6 patients (19.4\%) had a poor outcome (GOS 1) while predicted mortality according to PRISM III-24h was estimated to be $21.8 \%$. Among those with poor outcome, four cases of brain death due to secondary brain oedema were
Table

Demographic data *mean \pm SD

\begin{tabular}{lcc}
\hline & Group patients (all) & Group patients (deaths) \\
\hline GCS $^{*}$ & $8.9 \pm 4.3$ & $3.3 \pm 0.7$ \\
CT-scan $^{*}$ & $2.7 \pm 0.7$ & $3.6 \pm 0.5$ \\
ISS* $^{*}$ & $19.2 \pm 6.5$ & $28.3 \pm 2.5$ \\
PTS $^{*}$ & $3.2 \pm 2.8$ & $1.8 \pm 1.3$ \\
PRISM III-24h* & $16.2 \pm 8.3$ & $25.8 \pm 4.2$ \\
\hline
\end{tabular}

included. All fatalities were attributed to the severity of injury (Table). 5) In addition to GCS ( $r=0.78, P<0.01$ ) early hints for poor prognosis were acquired from $C T$ findings $(r=-0.84, P<0.01)$ and PTS $(r=0.57, P<0.01)$.

Conclusion: Despite the adverse environment related to the inherent impediments of an adult ICU, the results of this study are indicative of a rather efficient treatment of children with STBI compared to relative outcome results of specialised paediatric units.

\section{References:}

1. Feickert $\mathrm{HJ}$, Drommer $\mathrm{S}$, Heyer R: Severe head injury in children: impact of risk factors on outcome. J Trauma 1999, 47(1):33-38.

2. Ward JD: Pediatric issues in head trauma. New Horiz 1995, 3(3):539-545. 
P201 Outcome after major trauma: 12-month follow-up

A Anthi, S Georgopoulou, A Kalopissi, E Boukouvalas, K Mandragos and P Behrakis

Intensive Care Unit, Red Cross Hospital, Athens, Greece

Objective: The objective of this study was to describe the functional status of surviving major trauma victims after management on an ICU.

Patients and methods: During 1998, 86 trauma victim patients with an average injury severity score (ISS) of 32 , were admitted to the ICU of Red Cross Hospital in Athens. After ICU treatment, 50 patients survived and were discharged from the hospital. Follow-up 12 months later was completed for 41 patients (82\%). Functional capacity was assessed using Rosser's Disability Categories (RDC) .

Results: The mean age of surviving trauma victims was $36 \pm 16$ yrs. The mean ISS was $25 \pm 8$ and the mean length of ICU stay was $11 \pm 19$ days. Nine patients fell into Rosser's Disability Category 1, 10 patients into RDC 2, seven patients into RDC 3 , six patients into RDC 4, five patients into RDC 6, and four patients into RDC 7. No significant correlation was found between severity of trauma as expressed by ISS and Disability Categories.

Conclusions: $32 \%$ of survivors experienced severe social disability and a modest to severe work limitation (RDC $3,4)$. $22 \%$ of survivors were confined to a wheelchair or bed (RDC 6,7). 12-month follow-up after major trauma clearly demonstrates a profound level of functional limitation in more than $50 \%$ of patients. The severity of trauma alone does not seem to determine the functional capacity of surviving major trauma victims.

\section{P202 The Bair Hugger patient warming system in prolonged surgery}

JKC Huang, E Shah, N Vinodkumar, MA Hegarty* and RA Greatorex

Department of Surgery and *Department of Microbiology, Queen Elizabeth Hospital, Gayton Road, King's Lynn, Norfolk, PE30

$4 E T$, England, UK

Introduction: The Bair Hugger patient warming system is widely used and the advantages of avoiding hypothermia for patients undergoing surgical procedures are well established; however, prolonged exposure to the exhaust of the warming blanket may force the patients' skin organisms into the theatre atmosphere, and then into the surgical field and more importantly, onto prosthetic material such as vascular grafts, increasing the risk of devastating infection. This danger had not been previously studied.

Methods: Sixteen patients undergoing aortic surgery with graft insertion were studied. Air and wound specimens were taken from seven separate sites using standard methods with the centrifugal air sampler and agar touch plates. Two readings were taken from each site - one when the warming blanket was first applied at the start of the operation, and again at the end of the operation.

Results: The mean duration of surgery and use of the warming blanket was $234 \mathrm{~min}$ (180-270 min). Operating theatre air showed a significant decrease in colony counts at the end of surgery. The exhaust air colony counts remained similar. All wound specimens were sterile. None of the patients had postoperative wound-related infections.

Conclusions: The use of the Bair Hugger patient warming system during prolonged surgery does not lead to increased contamination of the surgical field nor increase the risk of wound and graft infection.

\section{P203 Induced moderate hypothermia markedly exacerbates pulmonary hypertension and dysoxia in a neonatal piglet model of elevated pulmonary vascular resistance}

MM Zayek, CR Hamm, KT O'Donnell and FG Eyal

Pediatrics, University of South Alabama, Mobile, AL, USA

Objective: To assess the cardiopulmonary effects of induced mild to moderate hypothermia in a neonatal animal model of pulmonary hypertension.
Background: Induced hypothermia has been proposed for neuroprotection following perinatal asphyxia. This latter condition is often associated with other pathophysiologi- 
cal derangements such as pulmonary hypertension, depressed cardiac function and decreased mesenteric blood flow. In those instances, hypothermia may become detrimental because of its potential to further increase pulmonary vascular resistance.

Design/methods: Anesthetized piglets ( $7 \pm 1$ days old, $\mathrm{n}=27$ ) were randomized to receive a thromboxane $A_{2}$ mimetic $(U 46,619,0.09 \pm .07 \mu \mathrm{g} / \mathrm{kg} / \mathrm{min})(\mathrm{TX})$ or nothing (CONT). One hour later, both groups were subjected to mild $\left(34-36^{\circ} \mathrm{C}\right)$ or moderate $\left(32-34^{\circ} \mathrm{C}\right)$ whole body hypothermia. Rewarming was started one hour later. At all times mechanical ventilation was modified to preserve normocapnea, $\mathrm{pH}>7.30$ and $\mathrm{PaO}_{2}>100$ torr. The following parameters were measured every 30 min: arterial, pulmonary and superior sagittal sinus blood gases; mean systemic (BP) and pulmonary artery pressures (PAP); cardiac output (thermodilution)(CO); internal carotid (CBF) and superior mesenteric (MBF) blood flow (ultrasonic flow transducers); lung mechanics and blood lactate concentration. Pulmonary (PVR) and systemic vascular resistance (SVR), alveolar-arterial oxygen gradient $\left(\mathrm{A}-\mathrm{aDO}_{2}\right)$, shunt fraction, oxygen index $\left(\mathrm{mPaw} x \mathrm{FiO}_{2} / \mathrm{PaO}_{2}\right)(\mathrm{Ol})$ and oxygen extraction and $\mathrm{O}_{2}$ consumption $\left(\mathrm{VO}_{2}\right)$ were derived from those parameters.

Results: Administration of TX increased $(P<0.01)$ baseline PVR by $137 \pm 7 \%$, while CO, MBF and CBF decreased by $23 \pm 4 \%, 33 \pm 1 \%$ and $29 \pm 7 \%$ respectively. The table shows the mean \pm s.e.m \% changes from their respective prehypothermia levels for each group at the end of the one hour hypothermic period.

Hypothermia caused a decrease in CBF (CONT: $25 \pm 8 \%$,TX:44 $\pm 9 \%)$, cerebral $\left(\mathrm{VO}_{2}\right) \quad(23 \pm 17 \%$ vs $34 \pm 11 \%)$ and MBF (23 \pm 7 vs $29 \pm 16 \%)$. CONT and TX were not statistically different. The physiological responses to mild or moderate hypothermia were similar. No excessive lactate production was observed.

Conclusions: Induced therapeutic hypothermia may exacerbate the adverse pulmonary hemodynamic effect associated with newborn pulmonary hypertension and lead to consequent hypoxemia.

\section{Table}

\begin{tabular}{lccccccc}
\hline$\%$ Changes: & $\uparrow \mathrm{AaDO}_{2}$ & $\uparrow$ Shunt F. & $\uparrow$ OI & $\uparrow \mathrm{PAP}$ & $\uparrow \mathrm{PVR}$ & $\uparrow \mathrm{PVR} / \mathrm{SVR}$ & $\downarrow \mathrm{CO}$ \\
\hline CONT & $21 \pm 18$ & $40 \pm 18$ & $11 \pm 6$ & $4 \pm 7$ & $42 \pm 12$ & $26 \pm 7$ & $-27 \pm 3$ \\
TX & $98 \pm 28$ & $220 \pm 70$ & $55 \pm 20$ & $27 \pm 4$ & $49 \pm 7$ & $53 \pm 7$ & $-16 \pm 3$ \\
R.M.ANOVA & $P<0.01$ & $P<0.01$ & $P<0.05$ & $P<0.01$ & & $P<0.01$ & $P<0.02$ \\
\hline
\end{tabular}

\section{P204 Accidental hypothermia and active rewarming: the metabolic changes observed above and below} $32^{\circ} \mathrm{C}$

JJ Mclnerney, B Breakell, W Madira, T Davies and PA Evans

Accident and Emergency department, The Leicester Royal Infirmary, Leicester, LE1 5WW, UK

Background: In accidental hypothermia the underlying physiological mechanisms responsible for poor outcome during rewarming through $32^{\circ} \mathrm{C}$ remain obscure. Deleterious changes in plasma calcium $\left(\mathrm{Ca}^{2+}\right)$, magnesium $\left(\mathrm{Mg}^{2+}\right)$, and acid-base concentrations have been reported in other forms of tissue injury, whilst parathyroid hormone (PTH) and interleukin 6 (IL-6) levels are elevated. Accordingly this study investigated the changes which occur in $\mathrm{Ca}^{2+}, \mathrm{Mg}^{2+}, \mathrm{pH}, \mathrm{PTH}$ and IL-6 during rewarming.

Methods: Eight patients, 4 males and 4 females, age 45 to 85 years, admitted with core temperatures $\angle 35^{\circ} \mathrm{C}$ were included in the study. Patients were rewarmed with dry warm blankets and fluid replaced by crystalloid at $40^{\circ} \mathrm{C}$. Blood for $\mathrm{Ca}^{2+}$ and $\mathrm{Mg}^{2+}, \mathrm{pH}, \mathrm{PTH}$ and IL-6 were collected at presentation, during rewarming, and at $24 \mathrm{~h}$.
Results: Four patients were admitted with mild hypothermia $\left(32-35^{\circ} \mathrm{C}\right)$ and four with moderate $\left(28-32^{\circ} \mathrm{C}\right)$ hypothermia. All patient clinical parameters responded favourably to rewarming. Rewarming to $32^{\circ} \mathrm{C}$ had no significant effect on the presenting acidosis $(P=0.1740)$, although above $32^{\circ} \mathrm{C} \mathrm{pH}$ increased with temperature $(P<0.0001)$. There was a negative correlation between $\mathrm{pH}$ and both $\mathrm{Ca}^{2+}(P=0.0005)$ and $\mathrm{Mg}^{2+}$ $(P=0.0488)$ below $32^{\circ} \mathrm{C}$; above this temperature the relationship was significant only for $\mathrm{Ca}^{2+}(P=0.0494)$. PTH and $\mathrm{Ca}^{2+}$ correlated positively $(P=0.0041)$ and negatively $(P=0.0039)$ in moderate and mild hypothermia respectively. IL- 6 and $\mathrm{Ca}^{2+}$ also correlated positively $(P=0.0039)$ and negatively $(P=0.0018)$ when presentation temperature was below and above $32^{\circ} \mathrm{C}$ respectively. 
Conclusions: During rewarming $\mathrm{pH}$ remains unchanged until patient temperature reaches $32^{\circ} \mathrm{C} . \mathrm{Ca}^{2+}$ and $\mathrm{Mg}^{2+}$ decline is associated with this $\mathrm{pH}$ change. Poor outcome is associated with presentation temperature $\left(<32^{\circ} \mathrm{C}\right)$, nonphysiological correlation between $\mathrm{PTH}$ and $\mathrm{Ca}^{2+}$, and age ( $\geq 84$ years). IL-6 levels mirror this PTH change but further study is required to explain these findings.

\section{P205 Treatment of severe heat stroke in an intensive care unit: clinical course, acute outcome and implications for management}

P Sorkine, O Szold, P Bidermann, S Bulocinic and P Halpern

General Intensive Care, Emergency Department Sourasky Medical Center. 6 Weizmann St. Tel Aviv 64239, Israel

Introduction: During the August 1998 heat wave in Tel Aviv, many patients were referred to the Tel Aviv Medical Center for acute heat-related illness. Of these, six patients were admitted to the Intensive Care Unit for severe heat stroke. We describe the clinical course during their first five days of hospitalization, response to treatment and implications for future management of this disorder.

Results: The six patients were admitted to the Intensive Care Unit in critical condition (mean APACHE II score $30 \pm 3.5)$, coma (mean Glasgow Coma 3.5 \pm 0.5 ), hypovolemic shock and respiratory failure necessitating mechanical ventilation. Despite early effective therapy (in all patients core temperature was reduced to less than $39^{\circ} \mathrm{C}$ in less than $1 \mathrm{~h}$ from admission to the hospital), one patient died (mortality 15\%) and four patients required further intensive care for life-threatening multiple organ failure.

Conclusion: During a heat wave such as that experienced in Tel Aviv during August 1998 a significant number of referrals for acute heat-related illness, possibly overwhelming the admission capacity of regional intensive-care units, must be anticipated. Severe heat stroke complicated by multiple organ failure may occur and is not necessarily related to physical activity prior to symptom onset. Although important in determining prognosis, early treatment does not prevent severe complications in this disorder. Mechanisms regulating body heat may remain disturbed for days following early treatment and apparent stabilization, mandating careful observation of ample duration in hospital.

\section{P206 Severe mushroom poisoning due to amatoxin in children}

\section{Uher, M Pisarcíková, J Filka, L Podracká and M Kurák}

University Hospital, Department of Pediatrics, PICU, Košice, Slovak Republic

Objectives: Mushroom poisoning is characterized by a very variable clinical picture. The amatoxins, the main toxic component of these fungi, are responsible for gastrointestinal symptoms as well as hepatic and renal failure. The authors present the clinical picture of the phalloid syndrome and its treatment.

Methods: Within a period of four years, 12 children with mean age of 10.75 years (4.5-16 years) including 6 males and 6 females with severe Amanita mushroom poisoning were treated at the PICU. The severity of poisoning was graded according to serum transaminase elevations and prolongation of prothrombin time. The diagnosis was based on anamnestic data, the clinical picture and, in the some patients, on mycological examinations of mushrooms residues, and their spores.

Results: The mean duration of hospitalization in the PICU was 8 days (3-19). 11/12 patients improved and were discharged from the hospital asymptomatic, one patient died. Our protocol for conservative treatment consisted of: fluid and electrolyte replacement, oral activated char- coal and lactulose, i.v. penicillin G, all patients received i.v. thioctic acid and i.v. silibinin, all received a special diet, and some patients received frozen fresh plasma and IV Fibrinogen. 3/12 children were given conservative therapy with hemodialysis and hemoperfusion This combination of treatment modalities was used to accelerate the elimination of amatoxin from the patients' bodies. The mean time between the consumption of the mushrooms and the first gastrointestinal symptoms was $9.84 \mathrm{~h}$ (4-18) and the mean time between the consumption and the admission to the hospital (the start of therapy) was $28 \mathrm{~h}$ (4-72). The duration of therapy according to the protocol of conservative therapy was 5.45 days (3.5-7.5). The increased liver enzymes SGPT and SGOT were found in 10/12 patients. In 7/12 patients the prothrombin time was prolongated. In 3/12 patients we used hemoperfusion combined with hemodialysis and in $2 / 12$ plasmapheresis.

Conclusions: We conclude that intensive combined treatment applied in our patients is highly effective in improving patients with both moderate and severe amanitin poisoning. 


\section{P207 Results of transplantation with kidneys from non-heart-beating donors}

\section{W Hordijk*, RGWL Tiggeler*, JA van der Vliet ${ }^{\dagger}$ and LB Hilbrands*}

*Department of Nephrology, ${ }^{\dagger}$ Department of Surgery, University Hospital Nijmegen "St. Radboud", P.0. Box 9101,6500 HB Nijmegen, The Netherlands

Introduction: During the last twenty years, thirty kidneys from non-heart-beating donors (NHBD) were used for transplantation in our hospital. We examined the results of these transplantations.

Methods: For every recipient from a NHBD kidney, we selected two recipients who received a kidney from a heart-beating cadaveric donor, as a control group (60). The control group was selected by matching the following items: first or retransplantation, HLA-AB or HLA-DR compatibility, cold ischaemia time, immunosuppressive medication and date of transplantation.

Results: According to "the Maastricht categories" classification from the NHBD resulted in: 1) death on arrival $(n=3) ; 2)$ unsuccessful resuscitation $(n=2) ; 3)$ awaiting cardiac arrest $(n=14)$; 4) cardiac arrest while brain dead $(n=11)$. The mean duration of follow-up was 4.5 years.
Graft survival rates at 1 and 5 years after transplantation were $87 \%$ and $57 \%$ respectively in the NHBD group and $85 \%$ and $72 \%$ in the control group (NS). The incidence of acute tubular necrosis(ATN) was $53 \%$ in the NHBD group and $30 \%$ in the control group $(P<0.05)$. With regard to graft function there was no difference between both groups. In transplantations with NHBD-kidneys, warm ischaemia time (WIT) shorter than 30 min was accompanied by a better graft survival than WIT longer than 30 min (5 year graft survival $81 \%$ versus $33 \%, P<0.05$ ). The incidence of ATN in kidneys with WIT shorter than $30 \mathrm{~min}$ was $40 \%$ versus $67 \%$ in kidneys with WIT of more than $30 \mathrm{~min}$ (NS).

Conclusion: Our results confirm that the use of kidneys from NHBD is a valuable contribution to a kidney transplantation program. A short WIT clearly leads to better results.

\section{P208 The role of selected biochemical values in graft quality prediction in brain death organ donors} V Cerny, P Zivny, R Parizkova and P Dostal

Department of Anesthesiology and Intensive Care, Charles University, Faculty of Medicine, 50005 Hradec Kralové, Czech Republic

Introduction: The aim of the study was to evaluate selected biochemical values in brain death kidney donors and to compare these results with number of rejection episodes in recipients.

Methods: After institutional approval 35 patients were prospectively studied. According to insult leading to brain injury, patients after brain death diagnosis (angiography) were divided into the two groups: donors with traumatic intracranial hemorrhage (Group D1) and donors with nontraumatic intracranial hemorrhage (Group D2). Blood samples were drawn immediately after brain death diagnosis. Lymphocyte count, CD3, CD4, CD8, CD19, CD56/16, CD25, ALT, AST, immunoglobulins (IgG, IgM, $\lg A)$, malondialdehyde, erythrocyte glutathione levels (GSH) and erythrocyte glutathione-peroxidase activity (GPX), selenium, slL-2R, IL-2, IL-6, IL-8 and TNF- $\alpha$ were measured. Results as mean (SD), ${ }^{\star} P<0,05$.

Results: Selected results are presented in the Table.

Conclusions: Selenium levels correlated with graft prognosis, significant differences in selected values were
Table

\begin{tabular}{lcc}
\hline & Group D1 $(\mathrm{n}=17)$ & Group D2 $(\mathrm{n}=18)$ \\
\hline Recipients no rejection & 8 & 7 \\
Rejection treated by steroids & 6 & 6 \\
Rejection resistant to steroids & 1 & 3 \\
AST $(\mu \mathrm{kat} / \mathrm{L})$ & $1.74 \pm 0.36^{\star}$ & $1.29 \pm 0.21$ \\
ALT $(\mu \mathrm{kat} / \mathrm{L})$ & $0.79 \pm 0.11^{\star}$ & $1.47 \pm 0.40$ \\
GPX $(\mathrm{mg} / \mathrm{g} \mathrm{Hb})$ & $44 \pm 5.4$ & $39.9 \pm 4.4$ \\
GSH $(\mathrm{mg} / \mathrm{g} \mathrm{Hb})$ & $2.84 \pm 0.3$ & $3.99 \pm 0.75$ \\
MDA $(\mu \mathrm{mol} / \mathrm{L})$ & $0.75 \pm 0.08$ & $0.66 \pm 0.09$ \\
Serum IL-6 $(\mathrm{pg} / \mathrm{mL})$ & $125.69 \pm 35.6$ & $146.9 \pm 41.6$ \\
Selenium $(\mu \mathrm{mol} / \mathrm{L})$ & $0.585 \pm 0.062$ & $0.761 \pm 0.077$ \\
\hline
\end{tabular}

found between trauma and non-trauma brain-stem death organ donors. High selenium levels in donors seem to be of value in prediction of later graft rejection. 


\section{P209 Experience with brain death and organ donation in a tertiary care hospital in Riyadh, Saudi} Arabia

W Djazmati ${ }^{*}$ B Attar', Y Arabi*, R Mclntyre* and FA Shaheen ${ }^{\dagger}$

*King Fahad National Guard Hospital, ICU 1425, PO Box 22490, Riyadh, 11426, Saudi Arabia and +Saudi Center for Organ

Transplantation (SCOT)

Introduction: The Kingdom of Saudi Arabia has a national organ procurement organization (SCOT) with rigid criteria for the definition of brain death and a standard method of approaching the families of potential organ donors. As one of the major ICUs in Riyadh, we have reviewed our experience with organ donation.

Methods: Retrospective review of all confirmed brain deaths in the 14 bed Adult ICU during the two-year period from January 1997 to December 1998.

Results: Out of 210 deaths in the ICU, there were 40 (19\%) confirmed brain deaths using the SCOT criteria. Mean age was $21.7 \pm 12.1$ years with male:female ratio of 9:1 reflecting the fact that $24(60 \%)$ of the cases resulted from road traffic accidents. Other trauma accounted for 4 (10\%), cerebro-vascular accidents for $7(18 \%)$ and brain anoxia for 5 (20\%). Thirty-one (78\%) patients were Saudi nationals and 9 (22\%) were non-Saudi. SCOT approached the relatives of all patients that were confirmed as brain dead and was successful in obtaining consent for organ donation from $14(35 \%)$ of these cases. Eight of these were Saudi and 6 were non-Saudi. Twentysix kidneys, 10 livers and 22 corneas were successfully transplanted and 13 hearts were used for valves.

Conclusion: 1) The organ retrieval rate of $35 \%$ compares favorably with other national and international data. This rate is probably related to the active involvement and coordination by SCOT. 2) Our incidence of brain death (19\%) is above average and possibly related to the high incidence of road traffic accidents due to the unique traffic conditions with extreme heat and lack of seat belt use. 3) Family refusal for organ donation remains the main reason for failure to donate and greater effort is still required to increase public awareness in the Kingdom about the concept of brain death and the importance of organ donation.

\section{P210 Epidemiology of cardiac arrhythmias in a medical-cardiologic intensive care unit: single center experience}

G Heinz, P Reinelt, G Delle Karth, A Geppert and P Siostrzonek

Department of Cardiology, University of Vienna, Austria

Aim of study: To obtain frequency and distribution of various types of significant, sustained arrhythmia (ARRHY), its time of occurrence and factors influencing the occurrence of ARRHY in a prospective observational study in a medical-cardiologic ICU.

Results: There were 310 ARRHY episodes in 133 patients (94 m, 39 f; age 65 \pm 12 years) during 11/1996 and 7/1999 (2.91 ARRHY episodes/patient, range 1-14). 278 episodes were tachyarrhythmias (TACHY; narrow-ORS complex [N-QRS] $n=108$, wide-QRS complex [W-ORS] TACHY $n=169$; regular $n=179$, irregular $n=98$ ) and 32 bradycardias. One case of primary ventricular fibrillation was classified as TACHY but not with respect to QRS width and regularity. The number of patients showing significant ARRHY was relatively constant over the years (1996: 4/28 [14.3\%], 1997: 50/302 [16.5\%], 1998: 51/286 [17.8\%], 22/140 (15.7\%) until 7/1999). 135/278 (48.6\%) W-QRS TACHY were assigned ventricular TACHY (VT). $12 \mathrm{~W}$-QRS TACHY episodes could not be classified with certainty. There were 13 episodes of Torsade de pointes (4.8\%). NQRS TACHY episodes were atrial fibrillation (AFIB) 83, atrial flutter 10, supraventricular TACHY 21, ectopic junctional TACHY 1. The occurrence of ARRHY followed a circadian pattern showing a peak during daytime and a decline
Figure 1

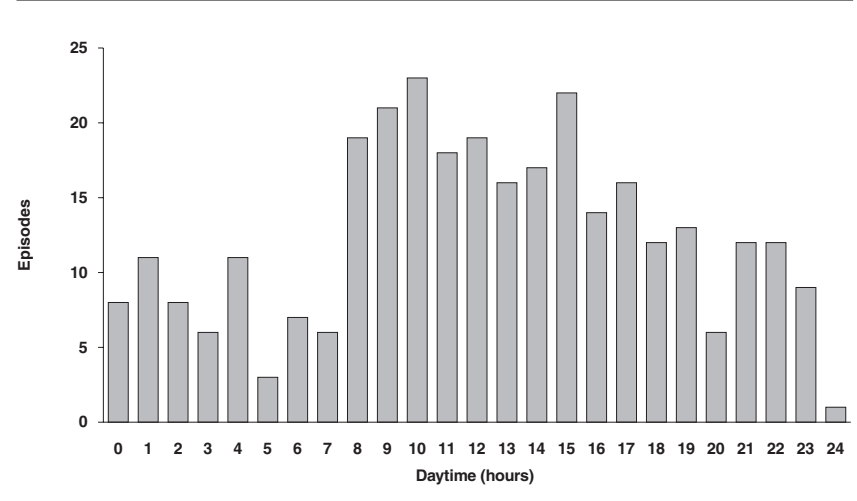

in the evening hours and early morning (Fig. 1). TACHY were treated electrically $(n=19)$, pharmacologically $(n=139)$ or combined $(n=77)$. The antiarrhythmic drugs most frequently used were amiodarone $(n=129)$, diltiazem $(n=47)$, ibutilide $(n=21)$, lidocaine $(n=24)$ and digitalis $(n=18)$. Proarrhythmia occurred due to haloperidol $(n=4)$, cisapride $(n=2)$, ibutilide $(n=1)$, and amiodarone $(n=4)$. Sedoanalge- 
sia $(P=0.64)$, mechanical ventilation $(P=0.76)$ or catecholamine treatment $(P=0.63)$ had no influence on the diurnal distribution of ARRHY. During 270/310 (90\%) episodes there was an elevated C-reactive protein (CRP), in $176 / 310(56.8 \%)$ an elevated leukocyte count [L] and in 230/310 (74\%) episodes were there elevated fibrinogen levels (FGEN). These inflammation parameters on the day of ARRHY did not differ significantly when compared to the respective values 24 and $48 \mathrm{~h}$ before ARRHY onset (CRP $16.6 \pm 11,16.3 \pm 11,16 \pm 1 \mathrm{mg} / \mathrm{dl}, P=0.9 ; \mathrm{L} 11.4 \pm 5,11.5 \pm 5$, $11.9 \pm 5.7 \quad \mathrm{G} / \mathrm{l}, \quad P=0.7 ; \quad \mathrm{FGEN} \quad 545 \pm 221, \quad 565 \pm 208$, $582 \pm 221 \mathrm{mg} / \mathrm{dl}, P=0.26$ ).
Conclusions: 1) Clinically significant, sustained ARRHY occurred in $\sim 1 / 5$ of patients in this medical-cardiologic $I C U$. 2) VT and AFIB were the single most frequent ARRHY. 3) ARRHY followed a circadian pattern irrespective of the presence of sedoanalgesia, mechanical ventilation or catecholamine support. 4) The vast majority of ARRHY occurred while there were signs of inflammation without preponderance to the ascending or descending limb of inflammation.

\section{P211 Transthoracic cardioversion with damped biphasic waveform shocks}

\section{VA Vostrikov, KV Razumov, PV Kholin and AL Cyrkin}

Department of Cardiology, Moscow Medical Academy, Hospital N1 and Hospital N 81, Moscow, Russia

Introduction: The biphasic waveform has been shown to have high efficacy for transthoracic ventricular defibrillation $[1,2]$. The objective of this prospective study was to evaluate the clinical efficacy of biphasic waveforms for cardioversion of atrial fibrillation.

Methods: The pulse is an asymmetric quasi-sinusoidal biphasic waveform. The peak current of the second phase was approximately half that of the first phase. Transthoracic cardioversion (29 emergent, 71 urgent and 41 elective) were performed in 141 patients who were receiving antiarrhythmic drugs (e.g., amiodarone). Ischemic heart disease was the most common (about 90\%) etiology. Shocks were delivered through $11.5 \mathrm{~cm}$ paddles in the antero-apical position. The maximum delivered energy was $195 \mathrm{~J}$.

Results: See Table.

\section{Table}

\begin{tabular}{lcc} 
Delivered Energy & Cumulative Success & $95 \%$ Confidence Interval \\
\hline$\leq 90 \mathrm{~J}$ & $113 / 141(80 \%)$ & $72 \%-87 \%$ \\
$\leq 140 \mathrm{~J}$ & $121 / 141(86 \%)$ & $79 \%-92 \%$ \\
$\leq 195 \mathrm{~J}$ & $132 / 141(94 \%)$ & $88 \%-97 \%$ \\
\hline
\end{tabular}

Conclusions: Our clinical results demonstrate that the biphasic waveform with a delivered energy of $\leq 195 \mathrm{~J}$ was highly effective in cardioverting atrial fibrillation.

\section{References:}

1. Poole J et al:: Resuscitation, 1998, 37(2):S13,(P-11).

2. Vostrikov $\mathrm{V}$ et al:: Resuscitation, 1998, 3(2):S42,(O-16).

\section{P212 Evaluation of transesophageal atrial pacing in the prone and lateral position}

\section{NM Schwann, DP Maguire, SE McNulty and JV Roth}

Departments of Anesthesiology, Thomas Jefferson University Hospital, Jefferson Medical College, and the Albert Einstein Medical Center, USA

Introduction: Transesophageal atrial pacing (TEAP) is used for temporary treatment of hypotension and/or low cardiac output caused by sinus bradycardia or atrioventricular junctional rhythm. It can also be used for temporary overdrive pacing of reentrant tachycardias. A pacing esophageal stethoscope (PES) is easy to place in supine intubated patients. However, no guidelines exist for PES placement in the prone or laterally positioned intubated patient.

Methods: After IRB approval and written informed consent, an $18 \mathrm{Fr}$ PES with $1 \mathrm{~cm}$ depth markings (Model 550 CardioCommand, Inc) was inserted to a depth of insertion (DOI) of $44 \mathrm{~cm}$ from the edge of the maxillary alveolar ridge into the esophagus of 30 adult intubated patients in the supine position. With the pacing rate set 10-20 beats per min faster than the patient's intrinsic heart rate and the current output on the pulse generator (Model 2A) set at its lowest current setting $(5.5 \mathrm{~mA})$, the current was gradually increased until atrial capture was achieved. The minimum current producing continuous atrial capture throughout the respiratory cycle was recorded as the pacing threshold. The PES was then withdrawn $1 \mathrm{~cm}$ at a time and pacing thresholds were redetermined for each DOI from 44 to $25 \mathrm{~cm}$ inclusive. After data collection in the supine position, each patient was then positioned into either the prone $(P)$, right (RLD) or left lateral decubitus (LLD) position. Data were collected in the same manner after the patients' position was changed. The width of the "Region of Capture" (ROC) represents 
the number of DOI locations where TEAP could be achieved with thresholds $40 \mathrm{~mA}$. The "Optimal" DOI represents where the minimum TEAP current threshold (THmin) occurred. Data is presented as mean \pm standard deviation.

Results: TEAP was accomplished in all 30 patients both in the supine and prone or lateral positions. The Optimal DOI increased on average only $1.2 \mathrm{~cm}$ (from 34.0 to 35.2 $\mathrm{cm})$ after positioning. Similarly, the average width of ROC increased only by $0.5 \mathrm{~cm}$ (from 12.4 to $12.9 \mathrm{~cm}$ ). The THmin (mean 14.4 $\pm 2.7 \mathrm{~mA}$ ) never decreased after placement of a supine patient into the prone position. It either remained unchanged or increased (minimum to maximum individual difference 0-8 mA). However, there was considerable variability in how the THmin, Optimal DOI, and ROC changed with the lateral position. Neither phrenic nerve pacing, brachial plexus stimulation, nor induction of arrhythmias was observed in any patient.

Discussion: This study demonstrates that TEAP with a PES can be successful in patients who are subsequently turned into the prone or lateral positions with minimal adjustments. TEAP may be initiated in the prone or lateral positions with the same insertion recommendations as those for patients in the supine position.

\section{P213 Cardiopulmonary resuscitation with cardiopulmonary bypass after cardiac surgery}

I Rodrigus, BJ Amsel, B Stockman, R De Paep and AC Moulijn

University Hospital Antwerp, Wilrijkstraat 10, 2650 Edegem, Belgium

Background: Emergency cardiopulmonary bypass (CPB) can be life-saving in cardiac arrest (CA) after cardiac surgery, when cardiopulmonary resuscitation (CPR) and open chest massage do not suffice. Outcome of these patients is, however, questionable. A retrospective study on these patients was done to investigate predictors of survival.

Material and methods: From January 1995 to October 1999, 18 patients experienced CA or ventricular fibrillation (VF) after a cardiac surgical procedure, refractory to CPR and open chest massage (i.e. $0.7 \%$ of our total adult cardiac surgery population). Mean age was 64.8 years (range 36-75) with 14/18 male patients. Preceding operative procedure was coronary artery bypass graft (CABG) $(n=1)$, urgent redo CABG $(n=2)$, ascending aorta replacement for dissection $(n=1)$. CA most often occurred some hours after surgery in intensive care $(n=14)$, or with the patient still in the operation theatre $(n=3)$. Duration of $\mathrm{CPR}$ is therefore always less than 1 hour. All patients were immediately put on conventional CPB. Events leading to CA were ischemia $(n=11)$, bleeding with or without tamponade $(n=3)$, aortic dissection, electromechanical dissociation, right ventricular failure or shock (each in 1 patient). In 8 of the 11 patients with ischemia, redo CABG was performed. In the other patients $(n=7)$, bleeding sites were handled or reperfusion was given.

Results: Of 8 patients with redo CABG as a resuscitative procedure, 5 could be weaned from CPB with or without intra-aortic balloon pumping (IABP) and survived. The remaining 3 patients were put on IABP $(n=1)$ or biventricular assist device (BVAD) $(n=2)$, but died. Three patients with reperfusion for ischemia did not survive, despite IABP $(n=2)$ or BVAD $(n=1)$. Of 7 patients without ischemia, only 2 survived.

Postoperative complications in the 7 surviving patients $(38 \%)$ were pneumonia $(n=5)$, renal failure with hemodialysis $(n=3)$, slow neurological recovery $(n=1)$. Hospital length of stay was 20.5 days (range 5-43). All but one patient are still in good health (mean follow up 26 months).

Conclusion: The incidence of CPR necessitating emergency CPB was low. Only aggressive treatment with emergent bedside resternotomy and CPB can save one third of these patients. Best results were achieved when a correctable cause for the CA was identified.

\section{P214 Functional status and quality of life (QoL) in long-term survivors of cardiac arrest after cardiac surgery}

A Anthi*, I Dimopoulou*, A Michalis ${ }^{\dagger}$ and GE Tzelepis*

*Intensive Care Unit and ${ }^{+2}$ 2nd Cardiac Surgery Department, Onassis Cardiac Surgery Center, Athens, Greece

We assessed long-term survival, functional status and QoL in cardiac surgery patients who survived after cardiac arrest during the immediate postoperative period. Of 3982 patients undergoing cardiac surgery over a 30 month period, 29 patients $(0.7 \%)$ had a sudden cardiac arrest. Of these, 27 patients (93\%) were successfully resuscitated with closed or open cardiopulmonary resuscitation (CPR). Overall, four patients (14\%) survived the
CPR but died in the ICU, and 23 patients (79\%) survived to hospital discharge. At reevaluation four years later, 16 patients were still alive, yielding an overall four-year survival rate of $55 \%$, while seven patients had died; of these last seven patients, three (43\%) died during the first year and the remaining at various time intervals. The New York Heart (NYHA) classification and a questionnaire based on the Nottingham Health Profile (NHP) were used to investi- 
gate functional status and QoL in these 16 long-term survivors. Of these, 12 patients (75\%) were in NYHA class I, three patients (19\%) in NYHA class II and one patient $(6 \%)$ in NYHA class III. None of the patient reported any emotional problems, signs of social isolation or difficulties in physical mobility. Six patients (38\%) had exertional angina and six patients (38\%) reported mild sleep disturbances, such as early awaking. All but two patients had retired (taking advantage of the available laws), 15 patients (94\%) were able to look after their home, 10 patients $(63 \%)$ had a sexual activity, 13 patients (81\%) were active with their special interests or hobbies, and 12 patients $(75 \%)$ had gone on holidays. In conclusion, more than $50 \%$ of post-cardiac surgery patients who survive after cardiac arrest occurring in the immediate postoperative period have a good functional status and a preserved quality of life up to 4 years following cardiac arrest and successful CPR.

\section{P215 Health-related quality of life after heart surgery}

JK Ohukainen, PK Kaukoranta and TI Ala-Kokko

Department of Anesthesiology, Oulu University Hospital, PL 22, 90220 Oulu, Finland

SF-36 health survey has been validated to measure QoL after intensive care [1]. A Finnish version of SF-36 is available with values for normal population [2]. This was used to evaluate QoL after heart surgery and postoperative intensive care.

Methods: Questionnaire was sent six months after ICU discharge to 454 patients who had undergone heart surgery during 01.01.1998-31.08.1998 in Oulu University Hospital.

Results: 375 patients, of whom 73\% were male, completed and returned the questionnaire. CABG was performed in $81 \%$ of the cases. $71 \%$ of the operations were elective. QoL of the patients was worse than that of the normal population, especially for physical functioning and general health. Instead general mental health and social functioning were at the same level. General well-being was worse than 12 months earlier in only $12 \%$ of the patients. Physical functioning $(P<0.001)$ and role limitations due to personal or emotional problems $(P=0.04)$ were worse in patients over 65 years old compared to those under 65. Pain worsened QoL more on younger patients $(P=0.04)$. Female gender $(P=0.002)$, emergency operation $(P<0.001)$, type of surgery $(P=0.02)$, neurological complications $(P=0.02)$ and preoperative HigginsCleveland classification $(P<0.001)$ were associated with low QoL. Instead postoperative infections, perioperative myocardial infarction and prolonged intensive care had no effect on QoL.

Conclusions: Most patients experience a better or equal QoL six months after heart surgery than before the operation. Better QoL can be achieved by optimising preoperative status and by elective surgery. Measures to avoid perioperative neurological complications must be applied to patient care.

\section{References:}

1. Chrispin PS et al.: Anaesthesia 1997, 52:15-23.

2. Hagman E: SLL 1996, 51:3534-3539.

\section{P216 Is postoperative ICU care needed for high-risk patients after major elective surgery?}

J Cohen, M Shapiro, J Sulkes*, E Grozovski and P Singer

${ }^{*}$ Department of General Intensive Care and Epidemiology, Rabin Medical Center, Beilinson Campus, Petah Tikva 49100;

Sackler School of Medicine, Tel Aviv University, Israel

Introduction: Patients are frequently admitted to ICU following elective surgery either because of surgery-specific or patient-specific risk. However, ICU beds are expensive and not always available. We assessed whether there were significant differences in outcome between patients admitted to an ICU and a similar group of patients managed in general surgical wards.

Methods: All patients undergoing elective surgery over a 17-month period and for whom a place in ICU was requested were included in this prospective study. The anesthetic and surgical departments were not made aware of the study. Following surgery, patients were either admitted to ICU if a bed was available (group 1, $n=97$ ) or transferred to the general surgical wards after a mean stay of
$1.9+3.7 \mathrm{~h}$ in the recovery room (group $2, \mathrm{n}=47$ ). We recorded preoperative factors (demographics, reason for

Table

\begin{tabular}{lccc}
\hline Variable & Group 1 $(\mathrm{n}=97)$ & Group 2 $(\mathrm{n}=47)$ & $P$ \\
\hline Surgery-specific risk & $59 \%$ & $38 \%$ & 0.02 \\
Serum creatinine $(\mathrm{mg} \%)$ & $1.4 \pm 0.76$ & $1.15 \pm 0.37$ & 0.012 \\
APACHE II & $9.8 \pm 4.2$ & $8.03 \pm 3.4$ & 0.02 \\
TISS & $19.86 \pm 4.3$ & $15.2 \pm 7.2$ & 0.001 \\
Ward complications & $34 \%$ & $13 \%$ & $\mathrm{NS}$ \\
Length of stay (days) & $11.9 \pm 6.9$ & $10.9 \pm 7.8$ & $\mathrm{NS}$ \\
28-day mortality & $15.4 \%(15 / 97)$ & $3.84 \%(4 / 47)$ & $\mathrm{NS}$ \\
\hline
\end{tabular}


admission, type of procedure, medical history, hemoglobin, serum creatinine, ASA, NYHA), intraoperative factors (type of anesthesia, intraoperative complications, units of blood transfused, length of surgery) and postoperative factors (duration of ventilation, APACHE II, TISS scores). Outcome variables were the occurrence of postoperative complications, duration of hospitalization and 28-day mortality.
Results: See Table.

Conclusion: We found no difference in three outcome variables in high-risk patients who were either admitted to the ICU or treated in a general surgical ward. Criteria for ICU admission need to be reassessed in these patients.

\section{P217 Abdominal sepsis in patients 65-onwards after non-scheduled laparotomy}

\section{P García-Saura, F Peramo, C Serrano, J Maldonado, A Márquez, MS Serrano and J Prieto}

Department of Anaesthesiology, Resuscitation and Pain Management, Hospital Clínico Universitario "San Cecilio", Granada, Spain

Introduction: Abdominal sepsis after emergency laparotomy has a high mortality rate because is associated with a great number of serious complications. The aim of this study was to assess abdominal sepsis associated mortality and to determine which variables could predict the possibility that abdominal sepsis occur.

Materials and methods: We prospectively studied 128 patients, 65 onwards, who underwent emergency laparotomy (abdominal resection, perforation or ischaemia). In every patient we recorded several variables of the pre, intra and postoperative period. By patient's history, radiographic and scan studies, laboratory tests specific for the type of surgery performed, wound inspection and surgical reexploration, we determine the presence of abdominal infection. We also studied mortality associated with this type of infection and variables that could predict its appearing (multiple logistic regression analysis, using a model in which probability an event $P$ will occur is $1 /\left(1+e^{z}\right)$, where $z$ is the linear combination, $z=B_{0}+$ $\mathrm{B}_{1} \mathrm{X}_{1}+\mathrm{B}_{2} \mathrm{X}_{2}+\ldots . \mathrm{B}_{\mathrm{P}} \mathrm{X}_{\mathrm{P}}$ B0, B1, $\mathrm{B}_{2}, \ldots \mathrm{B}_{\mathrm{p}}$ are coefficients estimated from the data, $\times$ is the independent variable and $\mathrm{e}$ is the base of the natural logarithms, approximately 2.718). Statistical analysis was made with SPSS for Windows $5.01^{\circledR}$ (multiple logistic regression analysis and
Fisher exact test as required) and $P<0.05$ was regarded as significant.

Results: We evaluated 52 women and 76 men and patients' characteristics were (mean \pm standard deviation): age $75.27 \pm 7.52$ and weight $70.34 \pm 16.35$. Abdominal sepsis was recorded in 15 patients (11.7\%) and 10 died (66.67\%). Mortality associated with abdominal infection was greater than mortality in patients without abdominal sepsis. Indeed, variables that can predict abdominal sepsis were diffuse vascular peripheral disease, acute renal failure, ARDS, surgical reexploration and a hospital stay greater than 7 days.

Conclusions: Emergency laparotomy has a greater mortality in patients with abdominal sepsis (66.67 vs $27 \%$ ). Variables that can predict its appearing are: decreasing $\mathrm{O}_{2}$ tissue delivery factors, events that increase the possibility of nosocomial infection or those circumstances favouring multisystemic organ failure (lung or kidney).

\section{References:}

1. Cook TM, Day CJE: Hospital mortality after urgent and emergency laparotomy in patients aged 65 years and over: Risk and prediction of risk using multiple logistic regression analysis. Br J Anaesth 1998, 80:776-781.

\section{P218 An experience with surgical admissions to a Paediatric ICU (PICU) in Harare Zimbabwe}

\section{I-E Pazvakavambwa}

Department of Paediatrics and Child Health, University of Zimbabwe, P.O. Box A178 Avondale, Harare, Zimbabwe

The PICU in Parirenyatwa Hospital in Harare is a 5bedded unit catering for both surgical and medical patients. A retrospective review was undertaken to evaluate the surgical ICU experience in the Unit. The study period was from January 1997 to December 1998.

Results: During this period there were 147 surgical admissions to the unit constituting just under half of the total admissions. The ages ranged from 1 day to 14 years with a median age of 36 months. Seventy-six (52\%) of the patients were male. The mean stay in the PICU was 3.9 days with a range of 28 days. Cardiac patients made up $45 \%$ of the patients followed by respiratory tract surgery
(15\%). The overall ICU mortality was $17 \%$. Twenty-five percent $(25 \%)$ of the patients were hypothermic on arrival to PICU. Factors associated with a high mortality were abnormal temperature on arrival in the PICU, young age and being ventilated. Three patients had accidental extubation on arrival to PICU.

Conclusions: There is a rather high incidence of hypothermia in surgical patients admitted to the PICU in Harare and this has adverse effect on mortality. Closer attention to temperature control during surgery and transport to PICU could reduce mortality. Further studies are needed in this regard. 


\section{P219 Organization and staffing of intensive care units in Brazil}

\section{J Livianu, JMC Orlando, A Giannini, RGG Terzi, M Moock, C Marcos and N David}

AMIB, r.Domingos de Morais 814 bloco Il cj.23, CEP 04010-100, São Paulo, SP, Brazil

Introduction: The ' $1^{\circ}$ Brazilian Census of ICUs' was developed by the Brazilian Society of Intensive Care (Associação Medicina Intensiva Brasileira - AMIB) to delineate the ICU profile in this country.

Methods: Data collection was done through a questionnaire sent to all hospitals. Through a software package, these data were gathered, creating a comprehensive database with ICU organizational and resource information.

Results: To be accredited as a training center by AMIB, the ICU must run a special program under specific conditions. At this census, $31.3 \%$ of the ICUs had a critical care training program but only $10.4 \%$ were accredited by AMIB. Despite the continental dimension of the country and the large number of units, they are concentrated (79\%) in south-east region. In Brazil, critical care medicine has largely been considered a second specialty by the physician: $61.1 \%$ were clinicians, $22.6 \%$ pediatricians, $9.8 \%$ surgeons and only $3.1 \%$ anesthesiologists. Just $23 \%$ of the intensivists are certified as specialists by
AMIB. $72.9 \%$ of the physicians work on duty (12 or $24 \mathrm{~h}$ shifts) and $18.1 \%$ work on a daily basis. $84.7 \%$ of the respondent ICUs had a chief-nurse exclusive to the ICU and $67.4 \%$ had therapists $12 \mathrm{~h}$ per day. $71.5 \%$ performed scientific meetings with ICU staff regularly. $51.5 \%$ had a computerized registry of admitted patients but only $33.4 \%$ classified admitted patients according to a scoring system. $74.8 \%$ had written admission and procedures rules and $64.4 \%$ had written therapeutic orientation rules. 83.3\% performed evaluation of adverse patient occurrences and $53.1 \%$ of the ICUs elaborated an annual report about their activities.

Conclusion: This study was the first step to recognize the structure and distribution of ICUs and exposed facts that must be improved, for instance, the need to increase the number of specialists through the creation of new training centers all over the country.

Acknowledgement: This study was supported by Bristol-Myers Squibb Brasil.

\section{P220 Baseline audit of manipulation and management of intravenous therapy delivery systems}

C Martinsen, A Hughes and M Smithies

Critical Care Services, University Hospital of Wales, Cardiff CF14 4XW, UK

Background: We are developing local evidence-based guidelines on the management of intravenous delivery systems in a 14-bedded Teaching Hospital General ICU1. A baseline audit was carried out to assess current practice prior to the publication of our proposed guidelines, and re-audit.

Methods: We performed an observational audit of the setup or change of an intravenous infusion and the management of intravenous delivery systems. ICU staff were aware that an observational audit was in progress but blind to what was being observed. The observations were carried out over a three-week period.

Results: See Table.

Conclusion: Practice is short of the stringent care necessary to avoid infection related to the delivery of intravenous therapy. The lack of consistency is also an issue. This audit highlighted the need for clinical guidelines and also the value of clinical audit of practice. Guidelines will now be introduced and developed in the light of appraisal of evidence, further audit and user feedback.
Table

\begin{tabular}{lcc}
\hline Set up or change of an intravenous infusion (n=13) & Yes & No \\
\hline Are hands washed before the procedure? & 1 & 12 \\
Are hands washed with soap and water? & 1 & 12 \\
Are hands washed for at least 15 s? & 0 & 13 \\
Is a clean plastic apron worn? & 8 & 5 \\
Are clinically clean gloves worn? & 8 & 5 \\
Was alcohol swab used prior to disconnection? & 0 & 13 \\
Was connection allowed to dry before disconnection? & 0 & 13 \\
Was set disposed of as unit policy? & 7 & 5 \\
Was alcohol swab used on valve prior to connection? & 0 & 11 \\
Was valve allowed to dry prior to connection? & 0 & 11 \\
\hline Set up or change of an intravenous infusion (n=10) & & \\
\hline Do all connections have a luer lock? & 32 & 0 \\
Are connected lines labelled? & 23 & 9 \\
Are all labelled lines within 72 h of date/time? & 6 & 28 \\
Do venous catheter hubs have Smartsite valve connected? & 33 & 0 \\
Do all catheters have a transparent dressings? & 13 & 1 \\
Are the catheter entry sites visible? & 12 & 5 \\
Does the dressing need to be changed? & 1 & 15 \\
Are all iv lines secured to the patient with tape? & 4 & 11 \\
\hline
\end{tabular}




\section{P221 Collaborative practice and clinical outcomes in the ICU}

\section{RE Nap, M Silva Alvaro, V Fidler and D Reis Miranda}

Health Services Research Unit, University Hospital Groningen, P.O. Box 30.001, 9700 RB Groningen, The Netherlands

Objective: To evaluate the effect of organizational and managerial intervention, aimed at increasing the professional collaboration between medical and nursing staff, upon the clinical outcomes in the ICU.

Design: Prospective, randomized, multi-center and multinational study.

Setting: 47 ICU's (22 control vs 25 intervention group) from 9 European countries.

Methods: All consecutive admissions were enrolled, during two periods of two months each.

Patient data: Admission data; first day SAPS II score; hourly registration of Critical Events (CrEv) defined as the duration (in hours) of out-of-range measurements of four parameters (heart rate, blood pressure, urine output and oxygen saturation); Sequential Organ Failure Score (SOFA) at admission and then every $24 \mathrm{~h}$; ICU outcome.

Measurements: Final outcome: ICU mortality. Intermediate outcomes: SOFA, CrEv (expressed as the \% of time spent in $\mathrm{CrEv}$; single or combined), and length of stay (LOS).

Intervention: 1) training of nursing and medical staff of 25 ICU's in inter-professional collaboration; 2) the use of a specific manual of instructions, supported by the daily use of two protocols covering awareness of processes of care, and professional dialogue (6 months).

Analysis: The effect of the intervention upon final and intermediate outcomes was evaluated by comparing the values of these variables in the experimental and the control groups of ICU's, before and after the intervention (Table below). SOFA computations included total daily score and $\operatorname{Delta}(\Delta)$-SOFA [1]. A $\Delta$-CrEv variable was also constructed. The analyses were made on Day 1,3 and 5 , in the group of patients staying $\geq 3$ and $\leq 10$ days in the ICU. $\chi^{2-}$ test was used for comparing means; significance if $P<0.05$

Results: Data on 1,633 patients were obtained; there were no significant differences in the 4 'study cells' for Age (median=69), SAPS \| (median=35) and LOS (median=4 days). A significant decrease in ICU mortality was observed for the intervention group (16\% control group; 9\% intervention group).

\begin{tabular}{llrrl} 
& & BI & I/C & $P$ \\
\hline Control group & SOFA day1 & $4.4 \pm 3.26$ & $4.3 \pm 3.35^{*}$ & NS \\
$\mathrm{n}=343$ vs 331 & SOFA day3 & $1.28 \pm 1.82$ & $1.56 \pm 2.21^{*}$ & NS \\
& SOFA day5 & $2.01 \pm 2.33$ & $2.09 \pm 2.51^{*}$ & NS \\
& CrEv day1 & $0.12 \pm 0.22$ & $0.13 \pm 0.20^{*}$ & NS \\
& CrEv day3 & $0.29 \pm 0.35$ & $0.36 \pm 0.44^{*}$ & NS \\
& CrEv day5 & $0.26 \pm 0.38$ & $0.28 \pm 0.40$ & NS \\
Intervention group & SOFA day1 & $3.70 \pm 3.33$ & $3.42 \pm 3.38$ & NS \\
$\mathrm{n}=603$ vs 356 & SOFA day3 & $1.33 \pm 1.95$ & $1.12 \pm 1.74$ & 0.00 \\
& SOFA day5 & $2.07 \pm 2.50$ & $1.66 \pm 1.98$ & 0.00 \\
& CrEv day1 & $0.11 \pm 0.17$ & $0.08 \pm 0.20$ & NS \\
& CrEv day3 & $0.35 \pm 0.45$ & $0.23 \pm 0.45$ & 0.000 \\
& CrEv day5 & $0.32 \pm 0.40$ & $0.21 \pm 0.48$ & 0.000
\end{tabular}

*Indicates significant differences between intervention and control groups. BI, before intervention; I/C, intervention/control.

Conclusion: Collaborative practice has a significant and beneficial effect upon clinical outcomes in the ICU.

Reference:

1. Moreno R et al.: Intensive Care Med 1999, 25:686-696.

P222 Prolonging ICU stay reduces the number of ward deaths: evaluation of a model for triaging ICU discharges

KJR Daly, RJ Beale and RWS Chang*

Department of Intensive Care, St Thomas' Hospital, London SE1 7EH; *Department of Renal Transplantation, St George's Hospital, London SW17 OQT, UK

Introduction: A significant number of patients discharged alive from the intensive care unit (ICU) die subsequently on the general wards. A predictive model using data from the patients' last day in the ICU prior to discharge, and a 0.6 cutoff, correctly identified $66 \%$ of ward deaths. We tested the model's ability to identify those patients who may benefit from a further 24 to 48 h stay in ICU.

Patients and methods: All ICU survivors discharged between 1st June 1990 to 31st December 1996 who stayed for more than three days, in whom the predictive model applied within $48 \mathrm{~h}$ of ICU discharge, were studied.
562 patients were classified into three groups: Group (0) 311 patients last predicted to be at risk of ward death on the day of ICU discharge; Group (1) 188 patients last predicted at risk $24 \mathrm{~h}$ prior to ICU discharge; Group (2) 63

Table

\begin{tabular}{lccc}
\hline Hospital outcome & $\begin{array}{c}\text { Group 0 } \\
\text { (333 patients) }\end{array}$ & $\begin{array}{c}\text { Group 1* } \\
(188 \text { patients })\end{array}$ & $\begin{array}{c}\text { Group 2** } \\
(63 \text { patients })\end{array}$ \\
\hline Alive & $256(82 \%)$ & $159(85 \%)$ & $59(94 \%)$ \\
Dead & $55(18 \%)$ & $29(15 \%)$ & $4(6 \%)$ \\
\hline
\end{tabular}

${ }^{*} \chi 2$ versus Group $0=$ not significant; ${ }^{\star \star} \chi 2$ versus Group $0 P<0.024$. 
patients last predicted at risk $48 \mathrm{~h}$ prior to ICU discharge. The model was further evaluated using another two independent data sets.

Results: See Table. Similar findings were found for the two other data sets.
Conclusion: There was a significant improvement in hospital survival for those patients who stayed in the ICU an additional $48 \mathrm{~h}$ following the prediction of ward death. If this can be confirmed in a prospective study, it will have a major impact on the provision of ICU beds in the United Kingdom.

\section{P223 Are we allocating limited resources to patients in most need?}

\section{T Nolin}

Intensive Care Unit, Hospital, 29185 Kristianstad, Sweden

Introduction: We aimed to examine this question by studying the correlation between severity of illness, outcome and the nurse workload (major determinant of cost).

Methods. We did a retrospective analysis of all intensive care patients admitted during 1992-1998 to the 10-bed general ICU. APACHE II [1] was used to determine the hospital mortality risk (MR). Patients were grouped into risk bands, in steps of $20 \%$. Standardised Mortality Ratio (SMR=observed hospital mortality/calculated hospital mortality) was used in each stratum to define clinical efficacy. As a proxy for resource consumption, a modified form [2] of the nursing care recording (NCR) system [3] was used. Workload per patient, per survivor, per non-survivor and 'effective' workload (workload all patients/ number of survivors) was calculated within each stratum.

Results: 4395 patients were admitted. 306 were children and 342 had missing values in scoring. APACHE II for sur- vivors/non-survivors was $12.7 / 23.4$, with estimated MR of 18.0/47.9\%. NCR per patient was 2.3 times higher for deceased (385) compared to survivor's (165). In survivors, NCR increased in a linear fashion with increasing MR up to $40<60 \%$. NCR in deceased patients was highest in those patients with lowest MR and lowest in those with highest MR. Effective work load increased gradually in a linear fashion with increasing MR.

Discussion. Resources were allocated to patients in most need. It was difficult to quantify the degree of efficacy. Effective NCR is an indicator of clinical efficacy in relation to severity of illness.

\footnotetext{
References:

1. Crit Care Med 1985, 10:818-829.

2. Acta Anaesthesiol Scand 1992, 36:610-614.

3. Svensk Anestesi och Intensivvård 1997, 1:16-38
}

\section{P224 Mortality and outcome in different subgroups of patients admitted in an ICU}

\section{A Marinho, J Luzio, F Prospero, L Lopes, M Fernandes, A Azevedo and M Brandão \\ Intensive Care Service, General Hospital St ${ }^{\circ}$ Antonio, Oporto, Portugal}

Introduction: SOFA and SAPS II scoring is used for monitoring of organ function and outcome prediction in intensive care. We evaluated the use of these scores to analysis the different subgroups of patients admitted to our ICU.

Methods: Over a period of nine months (between October 1998 and June 1999), the first five days scores of SOFA and SAPS II, were prospectively calculated for all consecutive patients (length of ICU stay $>12 \mathrm{~h}$ ) admitted to our 10-bed medical-surgical ICU. We stratified patients using three different subgroups: 1) medical (M); 2) surgical, elective (E); 3) surgical, unscheduled (U). Organ failure if SOFA score $\geq 3$. Mortality was assessed at ICU discharge. Data analysis and statistics were performed using the Statistical Package for Social Sciences (SPSS) version 6.0.1 for Windows.

Results: 278 patients, aged $58.5 \pm 18.4$ years, were included in the study. In this cohort of patients, the median length of ICU stay was $7.8 \pm 14.6$ days, SAPSII $29.1 \pm 13.5$ (median 27), SOFA $5 \pm 3.75$ (4), and the ICU mortality rate was $19.8 \%$. Relation between SOFA/SAPSII at admission and ICU discharged: non-survivors-SOFA 8.3 \pm 4.3 (8), SAPSIl 40.6 \pm 13.9 (39); survivors-SOFA 4.25 \pm 3.13 (3.5), SAPSII 26.3 \pm 11.8 (24.5). In the three different subgroups we have: $(\mathrm{M})-73$ patients $(26.2 \%)$, length of stay $13.78 \pm 20.98$ (median 6), SAPSII 33.9 \pm 12.6 (32), SOFA $5.37 \pm 3.7(4)$, mortality rate $30.1 \%$; (E) -150 patients $(54 \%)$ length of stay $3.89 \pm 8.05$ (1), SAPSII $23.3 \pm 10.2$ (22), SOFA 4.43 \pm 3.5 (4), mortality rate 10\%; (U)-55 patients $(19.8 \%)$ length of stay $10.47 \pm 15.47$ (5), SAPSII $38.7 \pm 4.07$ (38), SOFA $6.29 \pm 4.07$ (6), mortality rate $32.7 \%$. The mortality rate increased with the number of failing organs present at the time of admission (7.6\% with no organ failure, and $100 \%$ with 5 organs failure). The evaluation of a subgroup of 100 patients who stayed in the ICU for at least 5 days showed: length of stay $18.53 \pm 20.4$, SAPSII 35.49 \pm 10.9 , SOFA $6.76 \pm 3.75$ (7), mortality rate 34\%. Subgroups: (M)-57\%; (E)-25\%; (U)-28\%.

Conclusion: We have in our ICU two subgroups of patients: 1) medical (M) and unscheduled surgery (E) with 
higher length of stay, higher scores, and higher mortality rate, compared with the second group-elective surgery
(E). This is especially important when we try to compare the performance of different ICUs.

P225 Pre-admission functional status and outcome in medical intensive care

\section{Wehler, R Strauss, A Bost, A Geise, A Mueller, P Martus* and EG Hahn}

Departments of Medicine I and *Medical Informatics, Biometry and Epidemiology, University Erlangen-Nuremberg, PF 3560, 91023 Erlangen, Germany

Introduction: Guidelines for intensive care unit (ICU) admission recommend taking the premorbid functional status and comorbidity of patients into consideration when selecting those who are likely to benefit from ICU care [1]. We analyzed the prognostic value of pre-admission functional status, comorbidity and daily APACHE II scores in medical ICU patients.

Patients and methods: All patients $\geq 18$ years of age who stayed $>24 \mathrm{~h}$ in our non-coronary medical ICU were eligible for inclusion. Functional status referred to the two months prior to admission and was assessed using the domains of basic physiologic activities and activities of daily life of the PAEEC quality of life questionnaire [2]. Comorbidity was categorized using the Charlson Index (Cl) [3]. ICU, hospital and six-month follow-up mortality rates were documented. Statistical analysis used the cox model for censored data.

Results: During the 12-month study period, 325 patients were included. $55 \%$ were male, mean age was $58 \pm 17$ $( \pm S D)$ years, range 19-95 years. Mean ICU length of stay was $10.4 \pm 15$ days, range $2-127$ days. Mean APACHE II score (first $24 \mathrm{~h}$ ) was $23 \pm 10$, range $0-51.75 \%$ of our patients had one or more chronic diseases, mean $\mathrm{Cl}$ was $2.4 \pm 1.9$, range $0-11$. Functional status was reduced in $80 \%$ of our patients, with $40 \%$ of them demonstrating severe impairments. Cumulative mortality rates were: ICU 24\%, hospital 34.5\%, six-month follow-up 40.3\%. One patient was lost to follow-up. Multivariate analysis showed that pre-admission functional status (ICU/hospital mortality: $P=0.008 / P=0.0009), \mathrm{Cl}(P=0.003 / P=0.0005)$ and APACHE II score $(P=0.012 / P=0.012)$ had an highly significant association with ICU and hospital mortality.

Conclusion: In our population of critically ill medical patients with a high rate of preexisting chronic diseases, pre-admission functional status and comorbidity are independent variables with a highly significant association with on-unit and hospital mortality.

References:

1. Crit Care Med 1999, 27:633.

2. Rivera Fernandez R et al.: Intensive Care Med 1996, 22:1034.

3. Charlson ME et al.: J Chronic Dis 1987, 40:373.

\section{P226 Therapeutic activity in elderly medical intensive care patients}

\section{Wehler, R Strauss, A Mueller, U Reulbach*, P Martus* and EG Hahn \\ Departments of Medicine I and *Medical Informatics, Biometry and Epidemiology, University Erlangen-Nuremberg, PF 3560, 91023 Erlangen, Germany}

Introduction: Previous studies showed a limitation of therapeutic activity in elderly critically ill patients. We analysed whether the age of patients admitted to our medical intensive care unit (ICU) influenced the quantity of therapy provided.

Patients and methods: All patients admitted to our 12bed non-coronary medical ICU between 8/95 and 7/99 were enrolled in the study. Prospectively the reason for ICU admission, acute diagnoses, co-morbid illness, diagnostic procedures, daily APACHE II and TISS scores, ICU and hospital mortality rates were documented. Contingency tables were analysed using chi square test, continuous variables were compared using Mann-Whitney $U$ test.

Results: 2127 patients were studied; mean age was $57 \pm 18.6$ ( $\pm S D$ ) years, median 59 , range 18-101 years, $45 \%$ were female. Mean ICU length of stay was $7 \pm 12.8$ days, median 3. Mean APACHE II score (first $24 \mathrm{~h}$ ) was 18.6 \pm 12.3 . Mean TISS score (mean value of all days spent on the ICU) was 25.8 \pm 13.3 . ICU mortality was $23.6 \%$, hospital mortality was 31.7\%. 432 patients (20.3\%) were $\geq 75$ years of age. Compared with younger patients these patients had higher APACHE II scores (23.2 \pm 11.8 vs 17.5 $\pm 12.1, P=0.0005)$, higher modified APACHE II

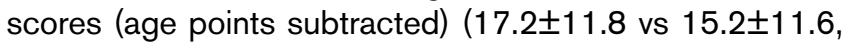
$P=0.001)$, higher mean TISS scores (28.3 \pm 12.2 vs $25.2 \pm 13.5, P=0.0005)$, a higher ICU mortality rate $(21.5 \%$ vs $31.7 \%, P=0.0005)$ and hospital mortality rate $(28.4 \%$ vs $44.4 \%, P=0.0005)$. No differences between the two age groups were noted in the number of days spent on a respirator or renal replacement therapy and in the frequency of diagnostic procedures, like ultrasound and endoscopy. Multivariate analysis showed that after adjustment for disease severity, mechanical ventilation and mortality rates patients $\geq 75$ years of age received the same amount of therapy as assessed by daily TISS points.

Conclusion: In contrast to other investigators we found no limitation of therapeutic activity in our sample of elderly medical ICU patients. 
http://ccforum.com/supplements/4/S1

\section{P227 Ward death following ICU discharge - can it be predicted? Development and validation of a predictive model}

KJR Daly, RJ Beale and RWS Chang*

Department of Intensive Care, St Thomas' Hospital, London SE1 7EH; *Department of Renal Transplantation, St George's Hospital, London SW17 OQT, UK

Introduction: A significant number of patients discharged alive from the intensive care unit (ICU) die subsequently on the general wards; a problem compounded by early discharge due to pressure on ICU beds. We analysed data from the patients' last day in the ICU prior to discharge, during their first ICU admission, to derive a predictive model that would identify those patients who were at risk of dying on the ward.

Patients and methods: All ICU survivors discharged between 1st June 1990 to 31st December 1996 were included in the study. Of the 5475 survivors there were 200 (3.7\%) ward deaths. Physiological and treatment data, collected prospectively from the Intensive Care Database (Riyadh Intensive Care Program, Medical Associated Software House Ltd, London, UK), for the patients' last day in the ICU were analysed to determine which variables had a significant influence on death following ICU discharge. Multiple logistic modelling was used to select which variables produced the 'best' predictive model of ward death. The model was then validated using two independent data sets.
Results: A higher mean acute physiology score (APS) (10.1 vs 8.0 ), increasing age (65 vs 59 years), presence of chronic ill health (39\% vs 22\%), longer ICU stay (8 vs 3 days), and whether the patient had cardiac surgery, were selected for inclusion in the model. The APS, age, chronic ill health and length of ICU stay were positively associated with death whilst having had cardiac surgery was positively associated with survival. Using a 0.6 cutoff the predictive model demonstrated good discrimination (sensitivity 66\%, specificity 88\%, ROC curve $85.6 \%$ ) and calibration ( $\left.\chi^{2} 7.5982 ; P=0.4737\right)$. External validation of the model produced a ROC curve of $86.8 \%$ (sensitivity $90 \%$, specificity $68 \%$ ) (data set 1 ) and $77.5 \%$ (sensitivity $75 \%$ specificity $69 \%$ ) (data set 2 ).

Conclusion: Using patient data obtained at ICU discharge it is possible to develop a discharge triage model which identifies those patients who are at risk of dying on the ward following ICU discharge.

P228 Withdrawing life-prolonging medical treatment in 246 patients

\section{T Nolin}

Intensive Care Unit, Hospital, 29185 Kristianstad, Sweden

Introduction: In this study, made in a 10-bed general ICU in a 500-bed district hospital, we examined 1) how often withdrawal (WD) decisions are made; 2) the reasons and; 3 ) the results of the decisions.

Methods: We followed up medical charts of 246 patients in whom we had documented decisions to withdraw lifesupport, according to our written policy. The study period was between 1994-1998.

Results: The incidence of WD decisions was 7.7\%. 41\% of patients dying in the ICU, and $40 \%$ of those later dying in the ward, had a decision made of WD. Of the 246 patients with a WD decision, 57\% died in ICU, 35\% later in the general ward and $8 \%$ were discharged alive from hospital. Median age was 76 years in those with a WD decision, as compared with 64 years in those without a decision. Reasons for WD of therapy were autonomy in $6 \%$, prognosis of acute disease in $34 \%$, prognosis of coexisting, chronic disease in 19\% and failure to respond to therapy in $41 \%$. Median time from admission to ICU to WD was 2.8 days, and from decision to death in ICU 1.9 days. In four cases renewed consideration was done. We found one case of discordance with relatives concerning the WD decision.

Discussion: Our results were similar to Sjøqvist et al. in Sweden [2] and show that 1) these decisions are common $(8 \%)$; 2) the reasons for the decision were mainly made from failure to respond to therapy and prognosis of the acute and underlying disease and; 3) few patients survive the hospital stay after such decisions.

\section{References:}

1. BMA: Withholding and Withdrawing Life-prolonging Medical Treatment. BMJ Books 1999.

2. Sjökvist $\mathrm{P}$ et al:: Limiting life support. Acta Anaesthesiol Scand 1998, 42:232-237. 
P229 Quality of life 6 and 12 months after discharge from the intensive care unit

HF O'Neill, GG Lavery, P Donnelly, M O'Mahony and B Alexander

Regional Intensive Care Unit, Royal Victoria Hospital, Belfast, BT12 6BA and Department of Psychology, The Queen's University of Belfast, Northern Ireland

Introduction: Mortality alone is a commonly used but nondiscriminating outcome measure after critical illness. Although we now view physical, psychological and social outcomes as important [1,2], which assessment tools to apply and when to apply them remains unresolved.

Method: Over a period of one year, we prospectively studied all patients who spent more than $72 \mathrm{~h}$ in a tertiary referral intensive care unit (ICU). All eligible patients were then visited by an ICU nurse at their normal residence 6 and 12 months after ICU discharge. They were scored using several scales: functional limitations profile (FLP), acceptance of illness (AOI), generalised self efficacy (GSES), health value (HVS), satisfaction with life (SWLS) and hospital anxiety (HAS) and depression (HDS).

Results: During the study period, there were 557 ICU admissions. ICU and hospital mortality rates were $21 \%$ $(n=119)$ and $32 \%(n=185)$. Study entry criteria were satisfied by 227 patients of whom 58 were excluded $(<18$ years old, no longer resident in region, refused to participate, died before interview). FLP scores (mean \pm SEM) for the 169 patients (M:F 99:70) at 6 and 12 months were $23.5 \pm 1.20$ and $18.2 \pm 1.03$ respectively, showing a signifi- cant increase in QOL $(P<0.001)$. AOI, HAS and GSES scores also improved between 6 and 12 months while HVS, SWLS and HDS were unchanged. The most significant factors determining $\mathrm{OOL}$ at both 6 and 12 months after discharge from ICU were levels of perceived physical and psychosocial limitation as measured by FLP. Age, admission APACHE II score and length of ICU stay did not influence $\mathrm{QOL}$ outcomes.

Conclusion. Measures of $\mathrm{QOL}$ outcomes in post-ICU patients vary with the assessment tool applied. Several show a significant increase in QOL between 6 and 12 months after ICU discharge and suggest that longer periods of follow-up than previously suggested [3] should be used.

\section{References:}

1. Rogers J. Ridley S. Chrispin P. Scotton H. Lloyd D: Reliability of next of kins' estimates of critically ill patients quality of life. Anaesthesia 1997, 52:1137-1143.

2. O'Boyle CA. Quality of life assessment: A paradigm shift in healthcare? The Irish Journal of Psychology 1997, 18:51-66.

3. Bell $D$ and Turpin K. Quality of life at three months following admission to intensive and coronary care units. Clinical Intensive Care 1994, 5:276-281.

P230 Change in the provision of out-of-hours consultant cover improves case-mix adjusted mortality in a district general (university-affiliated) hospital intensive care unit

MC Blunt and KR Burchett

Queen Elizabeth Hospital, Gayton Road, King's Lynn, Norfolk, PE30 4ET, UK

Introduction: In the United Kingdom the national recommendations [1] for intensive care units (ICUs) include the 24-h availability of consultants with a sessional commitment to intensive care (intensivists). In practice this has not been possible outside the larger teaching hospitals, and normal practice in other hospitals has been for the consultant anaesthetist to provide cover for the ICU out of routine hours. Following introduction of 24-h intensivist cover in our hospital we wished to assess whether there was an improvement in mortality standardised for case-mix using the APACHE 2 prognostic calculation [2] (SMR).

Methods: The 465 patients admitted to ICU in the 18 months following introduction of 24-h intensivist cover (Intensivist) were compared with the 387 patients admitted to ICU in the 18 months immediately preceding the change (Non-Specialist) in a historically controlled study. APACHE 2 scores, calculated risk of death, age, ICU lengths of stay and hospital length of stay (survivors) were collected from the ICU database, and SMRs were calculated for each group. Patients who were under 16 years of
Table

\begin{tabular}{lcc}
\hline & Intensivist & Non-Specialist \\
\hline Age (years) & $57.6(55.6-59.6)$ & $59.7(57.7-61.7)$ \\
APACHE 2 & $18.1(17.4-18.8)^{\star}$ & $19.3(18.5-20.1)$ \\
Risk of Death (\%) & $29.6(27.4-31.8)$ & $31.0(28.5-33.5)$ \\
ICU length of stay (days) & $1.5(0.1-6.6)$ & $1.8(0.2-5.3)$ \\
Time to hospital & $13(4-23)$ & $14(4-19)$ \\
discharge (days) & & \\
Standardised Mortality & $0.81(0.66-0.98)^{\star \star}$ & $1.11(0.93-1.34)$ \\
Ratio (95\% Cl) & &
\end{tabular}

Data presented as mean (95\% Confidence intervals) for age, APACHE and risk of death; median (interquartile range) for time data.

${ }^{*} P<0.05 ;{ }^{* *} P<0.01$.

age or who stayed in ICU<8 h (Intensivist: 49; Non-Specialist: 59) were excluded. Demographic data was assessed using ANOVA and SMR by Poisson distribution.

Results: There was a significant improvement in SMR in the intensivist group $(P<0.01)$. The APACHE 2 scores in 
the patients in the intensivist group were significantly lower $(P<0.05)$ (Table).

Conclusions: Within the methodological restrictions of the historical control design this study supports the introduction of 24-h intensivist cover in all intensive care units. The use of SMR as the primary end-point ensures a mean- ingful comparison of the groups despite the lower APACHE 2 scores in the intensivist group.

\section{References:}

1. Standards for Intensive Care Units. Intensive Care Society; London. 1997.

2. Rowan KM, Kerr JH, Major E, McPherson K, Short A, Vessey MP. BMJ 1993, 307:972-977.

\section{P231 A comparison between some standardized scales of evaluation of anxiety and depression in nursing staff}

D Chiumello, P Caironi, P Pelosi ${ }^{\star}, \mathbf{S}$ Losappio, M Mondino, R Malacrida ${ }^{\dagger}, \mathbf{M}$ Tomamichel§ and L Gattinoni

1st. di Anestesia e Rianimazione, Università degli Studi, Osp. Maggiore di Milano-IRCCS, Italy; *Dip. di Scienze Cliniche e Biologiche, Università dell'Insubria, Varese, Italy; ${ }^{\dagger}$ Ospedale Civico di Lugano, Switzerland

Anxiety and depression can be evaluated through different methods. We studied the current associations between the commonest evaluation methods that are present in scientific literature.

Materials and methods: We analyzed a population of 1392 nurses working in intensive care units (ICUs) and in general medicine units (GMUs), distributed in 101 Italian hospitals (with a mean age of $32 \pm 6.9$ years, $73.8 \%$ female). We considered the following evaluation scales: the Hospital Anxiety and Depression scale, divided in anxiety (HAD A) and depression (HAD D) status; the STAl scale, divided in acute anxiety ( $\mathrm{Y}-1)$ and chronic anxiety ( $\mathrm{Y}-2)$ status; the Maslach Burnout Inventory-Human Services Survey (MBI.), divided in Emotional Exhaustion (EE), Depersonalization (DP) and Personal Accomplishment (PA). Assuming the HAD as a reference scale, we evaluated the influence of the others scales to determine HAD. The population was divided, distinctly for anxiety and depression, into three groups, according to standardized parameters of HAD - 'non-cases' (HAD 0-7), 'doubtful cases' (HAD 8-10), and 'cases' (HAD 11-21). We used multiple linear regression models; statistical significance was accepted as $P<0.05$.

Results: The regression coefficients of the multiple linear regression models are expressed in the table, with the variables that result in statistical significance. For depression, we considered doubtful cases and cases together (last being only 39).

Conclusions: 1) Besides HAD A, STAI Y-2 also seems to be useful for evaluating anxiety, whatever the degree of it. 2) Besides HAD D, STAI Y-1 and MBI EE also seem to be useful for evaluating depression, whatever the degree of it.
Table

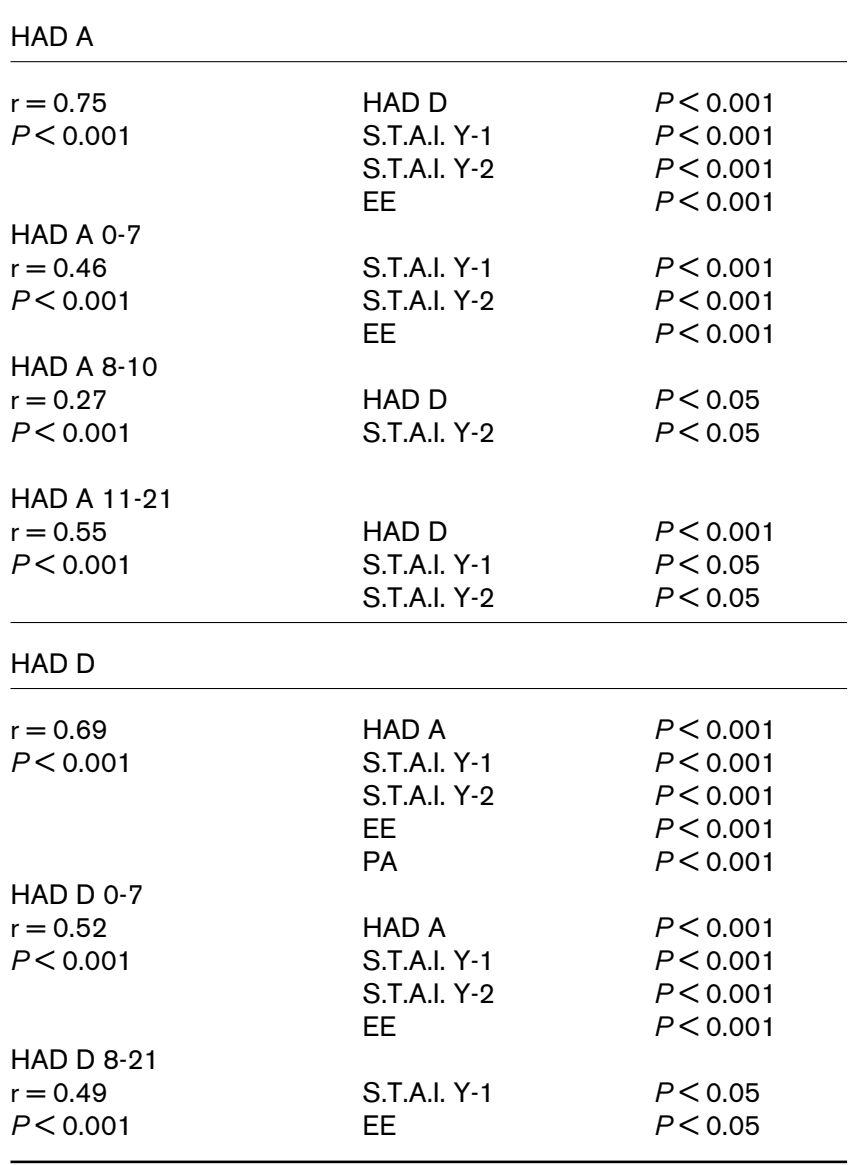

\section{P232 Stress in nursing staff: a comparative analysis between intensive care units and general medicine units}

D Chiumello, P Caironi, P Pelosi ${ }^{\star}$, S Losappio, R Malacrida ${ }^{\dagger}, \mathbf{M}$ Tomamichel§ and L Gattinoni

1st.. di Anestesia e Rianimazione, Università degli Studi, Osp. Maggiore di Milano-IRCCS, Italy; ${ }^{*}$ ip. di Scienze Cliniche e Biologiche, Università dell'Insubria, Varese, Italy; ${ }^{\dagger}$ Ospedale Civico di Lugano, Switzerland

It's a current belief that stress is an outstanding feature of intensive care units, in particular within nursing staff. The aim of this study was to compare some variables belong- ing to stress (i.e. anxiety, depression and 'Burnout' syndrome) between nurses working in intensive care units (ICUs) and general medicine units (GMUs). 
Table 1

\begin{tabular}{lccc}
\hline & ICU & GMU & \\
\hline HAD A & $7(5,9)$ & $7(5,10)$ & $P<0.005$ \\
A 11-21 & $13 \%$ & $19 \%$ & $P<0.005$ \\
HAD D & $3(2,6)$ & $4(2,6)$ & n.s. \\
S.T.A.I. Y-1 & $33(30,39)$ & $35(31,41)$ & $P<0.005$ \\
S.T.A.I. Y-2 & $36(31,42)$ & $37(32,43)$ & $P<0.05$ \\
M.B.I. EE & $19(13,29)$ & $25(16,34)$ & $P<0.001$ \\
EE $\geq 27$ & $29 \%$ & $42 \%$ & $P<0.001$ \\
M.B.I. DP & $6(2,11)$ & $6(1,11)$ & n.s. \\
M.B.I. PA & $36(29,41)$ & $37(32,41)$ & $P<0.001$ \\
A1 & $5(3,7)$ & $6(4,8)$ & $P<0.001$ \\
A2 & $6(3,8)$ & $5(2,7)$ & $P<0.001$ \\
A3 & $4(2,6)$ & $4(2,6)$ & n.s. \\
A4 & $4(2,6)$ & $7(5,9)$ & $P<0.001$ \\
A5 & $5(2,7)$ & $7(4,9)$ & $P<0.001$ \\
A6 & $3(1,5)$ & $5(3,7)$ & $P<0.001$ \\
A7 & $5(2,7)$ & $5(2,8)$ & $P<0.05$ \\
\hline
\end{tabular}

Materials and methods: We studied a population of 883 nurses working in ICUs, distributed in 79 Italian hospitals (70.1\% female) and 509 nurses working in GMUs, distributed in 35 Italian hospitals (80.2 \% female). We asked them to fill in a form including: 1) general data and his/her work environment; 2) different evaluation standardized scales - the Hospital Anxiety and Depression Scale, divided into anxiety (HAD A) and depression (HAD D) status 0-7 'non cases', 8-10 'doubtful cases', 11-21 'cases'; the S.T.A.I. scale, divided into acute anxiety (Y-1) and chronic anxiety (Y-2) status; the Maslach Burnout Inventory-Human Services Survey (MBI.) divided into Emotional Exhaustion (EE), $\leq 18$ 'low', 19-26 'average', $\geq 27$ 'high', Depersonalization (DP) and Personal Accom-
Table 2

\begin{tabular}{lccc}
\hline & ICU & GMU & \\
\hline Beds/unit & 8 & 35 & $P<0.001$ \\
Admissions/year & 352 & 1291 & $P<0.001$ \\
Nurses/unit & 19 & 15 & $P<0.001$ \\
Physicians/unit & 12 & 7 & $P<0.001$ \\
Age & 32 & 30 & $P<0.001$ \\
Hours/week & 36 & 37 & n.s. \\
Nights/month & 6 & 5 & $P<0.005$ \\
Patients/nurse & 2 & 29 & $P<0.001$ \\
\hline
\end{tabular}

plishment (PA). We also evaluated the different reasons of anxiety through individual questions (higher value, more anxiety): $A 1$, a critically ill patient; $A 2$, a young patient; $A 3$, an old patient; $A 4$, a suicidal patient; $A 5$, a terminal patient; $A 6$, presence of mechanical supports; 7 7, relationship with patients' relatives. The comparison between the two groups was performed by the Mann-Whitney Rank Sum test and z-test; statistical significance was accepted as $P<0.05$.

Results: The results, expressed as median value, with 25th and 75th percentile in brackets, are shown in Tables 1 and 2.

Table 1 also shows the proportions of nurses that had a highest value of HAD A and M.B.I. EE.

Conclusions: Pathologic anxiety and emotional exhaustion are more prevalent in nurses working in GMUs. Thus, contrary to a common belief, 'stress' is a more distinctive peculiarity of general medicine units than intensive care units.

\section{P233 Anxiety and depression in family members of ICU patients: ethical considerations regarding decision-making capacity}

F Pochard, E Azoulay, S Chevret, F Lemaire, P Hubert, P Canouï, JR Le Gall, JF Dhainaut and B Schlemmer

For the FAMIREA group, Saint-Louis hospital and University Paris 7, 1 av. C Vellefaux, 75010 PARIS, France

Background: Anxiety and depression have a major impact on the ability to make decisions. Characterization of symptoms reflecting anxiety and depression in family members visiting ICU patients may be of major relevance to the ethics of involving family members in decision-making, particularly about end-of-life issues.

Methods: Prospective multi-center study in 43 French ICUs (37 adult and 6 pediatric). Each unit included 15 patients admitted for longer than two days. ICU characteristics and data on the patient and family members were collected. Family members completed the Hospital Anxiety and Depression Scale (HADS) to allow evaluation of the prevalence and potential predictors of anxiety and depression.

Findings: 637 patients were included in the study and 920 family members completed the HADS. All items were completed in 836 HADS questionnaires, which formed the basis for this study. The prevalences of anxiety and depression in family members were $69.1 \%$ and $35.4 \%$, respectively. Anxiety or depression were present in $72.7 \%$ of family members and $84 \%$ of spouses. Factors predictive of anxiety in a multivariate model included patientrelated factors (absence of chronic disease), family-related factors (spouse, female gender, desire for professional psychological help, help being received by usual doctor) and caregiver-related factors (absence of physician-nurse meetings on a regular basis, absence of a room used only for meetings with family members). The multivariate model also identified three groups of factors predicting depression: patient-related (age), family-related (spouse, female gender, not of French descent), and caregiver-related (no waiting room, perceived contradictions in the information provided by caregivers). 
Interpretation and conclusion: More than two-thirds of family members visiting ICU patients suffer anxiety or depression. Their involvement in end-of-life decisions should be carefully discussed.
Acknowledgement: This abstract is funded by the French Society of Critical Care Medicine.

\title{
P234 Meeting the needs of ICU patient families: a multicentre study
}

\author{
E Azoulay, F Pochard, S Chevret, F Lemaire, M Mokhtari, J R LeGall, JF Dhainaut and B Schlemmer \\ For the FAMIREA group, St-Louis hospital and University Paris 7, 1 av. Vellefaux, 75010 PARIS, France
}

Background: ICU caregivers should specifically seek to develop a collaborative relationship with ICU patient family members, based on a rich and free exchange of information and aimed both at helping family members to cope with their distress and at providing them with the opportunity to speak for the patient if necessary.

Methods: We conducted a prospective multicentre study of family member satisfaction as evaluated using the Critical Care Family Needs Inventory (CCFNI) developed by the Society for Critical Care Medicine. Forty-three French ICUs (37 adult and 6 paediatric) participated in the study; each was required to include 15 consecutive patients hospitalised for longer than two days. ICU characteristics, demographic data on patients and family members and questions about satisfaction were collected. The additive score of all CCFNI items was the dependent variable. Factors associated with dissatisfaction were identified using multivariate analysis.

Results: 637 patients were included in the study, and 920 family members completed the questionnaire. The multiple regression model identified 10 factors associated with family dissatisfaction: (i) two patient-related factors, namely age (OR 1.23) and admission for acute respiratory failure (OR 1.62); (ii) three family-related factors, namely younger age (OR 0.98), not the spouse (OR 1.81), and not of French descent (OR 4.34); and (iii) five caregiverrelated factors, namely perceived contradictions in information given by caregivers (OR 3.20), information not always provided by the same physician (OR 2.71), no knowledge of the occupational identity of each caregiver (OR 2.32), no help from the family's own doctor (OR 1.45 ), and insufficient caregiver time spent giving information (OR 1.35).

Conclusion: Among family members visiting the patient, spouses were more satisfied. The caregiver-related factors identified in this study are amenable to intervention and deserve to be the focus of future studies aimed at improving the satisfaction of ICU patient family members.

Acknowledgement: This abstract is funded by: the French Society of Critical Care Medicine.

\section{P235 Acute psychological reactions in relatives of patients treated in the intensive care unit (ICU)}

A Melissaki*, T Paparrigopoulos ${ }^{\dagger}$, A Efthymiou*, Ch Vadala*, G Kribeni* and E Pavlou*

*ICU, Agios Savvas General Hospital, 171 Alexandras Ave., GR - 11522, Athens, Greece; ${ }^{\dagger}$ Athens University Medical School, Department of Psychiatry, Eginition Hospital, ₹ICU, KAT General Hospital, Greece

Objective: The objective of the present work was the investigation of the impact of admission and treatment of patients in the intensive care unit (ICU) on their relatives, by measuring their acute psychological reactions. More explicitly, to disclose if ICU treatment acts as a stressor of a potentially traumatic nature to the patient's relatives.

Material and methods: The sample comprised of 48 subjects (24 males/24 females) who were close relatives of patients treated in the ICU of Agios Savvas and KAT General Hospitals for various reasons over a six-month period. Demographic data regarding both the patients and their relatives were collected. Information on the physical condition of patients (type of operation, APACHE II Score, assisted ventilation etc.) was obtained from their medical report. The instruments applied to assess the psychological impact of ICU admission on the patient's relatives were the Center for Epidemiological Studies Depression Scale (CES-D), the Spielberger's State-Trait Anxiety Inventory
(STAI) and the Impact of Event Scale (IES), which is a measure of intrusion and avoidance. Subjects were assessed one week \pm one day after their relative's admission to the ICU.

Results: Patients were admitted to the ICU for various reasons (injuries, 11; head injury, 12; postoperative care, 8; various emergencies, 17). Mean age of patients was 48.6 \pm 19.5 years (min, 17; max, 81); mean APACHE ॥ Score upon admission was $12.8 \pm 5.0(\min , 2 ; \max , 24)$. Mean age of relatives was $40.5 \pm 12.3$ years ( $\mathrm{min}, 21$; max, 73). They were first degree relatives; $70 \%$ were married and had children. Mean scores $\pm S D$ on the psychometric scales were as follows: CES-D $=29.8 \pm 8.9$, STAIState $=60.4 \pm 13.2, \quad$ STAI-Trait $=39.8 \pm 9.0, \quad$ IES-Total $=$ $26.15 \pm 7.9$, IES-Intrusion=16.0 \pm 4.7 , IES-Avoidance= $10.1 \pm 4.8$. These scores indicate the presence of severe depressive symptoms, high levels of subjective tension and apprehension, and moderate levels of intrusion and 
avoidance. Females scored significantly higher than males on all scales (Mean $\pm S D$ and t-test 2-tail sig: CES-D= $32.8 \pm 7.4$ vs $26.8 \pm 9.2 P=0.02$; STAl-State $=65.5 \pm 10.8$ vs $55.3 \pm 13.5 \quad P=0.006 ; \quad$ STAl-Trait $=42.6 \pm 8.6$ vs $36.8 \pm 8.7 P=0.025$; IES-Intrusion $=17.6 \pm 3.6$ vs $14.5 \pm 5.2$ $P=0.02$; IES-Avoidance $=11.1 \pm 5.0$ vs $9.0 \pm 4.5 P=0.13$ ). Spearman correlation coefficients were also computed between the different scales that were found to be highly correlated (level of sig. >0.01). Stepwise multiple linear regression of CES-D upon various independent variables (i.e. age of patient, ICU diagnosis, APACHE II Score, age and gender of relative, degree of kinship, relative's socioeconomic and marital status, and the score on STAI-Trait inventory) showed that the severity of the depressive symptomatology depends significantly solely on the relatives STAI-Trait score.

Conclusions: The main findings of the present study are: a) treatment in the ICU acts as a high impact stressor for the patient's relatives, equivalent to other traumatic events of an exceptionally threatening nature; b) females are significantly more prone than males to develop a severe anxiety-depressive reaction; they also experience more intense intrusive symptoms; and c) individuals with higher pre-exposure anxiety levels, particularly females, are more vulnerable to the development of an acute stress reactiontype symptomatology.

\section{P236 Severity of illness, critical events, organ failure assessment and ICU outcome}

\section{Silva Alvaro, RE Nap, V Fidler and D Reis Miranda}

Health Services Research Unit, University Hospital Groningen, P.O. Box 30.001, 9700 RB Groningen, The Netherlands

Background: The use of general outcome prediction models in the ICU remains controversial: 1) none of the existing systems is able to precisely predict individual outcome; 2 ) based on data collected within the first $24 \mathrm{~h}$ after admission, their prediction power is insensitive to alternative courses of care (and of ways-of-working) in the ICU.

Objectives: To evaluate the importance of intermediate outcomes of care upon the final outcome of patient care in the ICU; to evaluate whether the consideration of intermediate outcomes of care do increase the predictive power of SAPS II score.

Design: Prospective, multi-center and multinational study.

Setting: 47 ICU's from 9 European countries.

Methods: All consecutive admissions were enrolled during a four-month period.

Patient data: admission data; first day SAPS II score; hourly registration of Critical Events (CrEv) defined as the duration (in hours) of out-of-range measurements of four parameters (heart rate, blood pressure, urine output and oxygen saturation); Sequential Organ Failure Score (SOFA) at admission and then every $24 \mathrm{~h}$; ICU outcome.

Measurements: Final outcome: ICU mortality. Intermediate outcomes: SOFA and CrEv, expressed as the percentage of time spent in $\mathrm{CrEv}$ (single or combined). SOFA computations included total daily score and Delta-SOFA [1].

Analysis: variables included for explaining final outcome (the dependent variable) on Day 3 through Day 10: Day 1 SAPS II score; intermediate outcome variables (SOFA and CrEv) on Day 1, Day 3 and Day 5.
Table

\begin{tabular}{ccccc}
\hline & \multicolumn{5}{c}{ Relative } \\
& Mean \pm sd & Risk & $95 \% \mathrm{Cl}$ & $P$ \\
\hline Model I SAPS II & $36.4 \pm 16.3$ & 1.04 & $1.0302-1.0526$ & 0.0000 \\
SOFA day 1 & $4 \pm 3.4$ & 1.2 & $1.0989-1.211$ & 0.0000 \\
CrEv day 1 & $0.1 \pm .2$ & 3.12 & $1.6787-5.7809$ & 0.0003 \\
Model II SAPS II & $36.4 \pm 16.3$ & 1.0480 & $1.0376-1.0586$ & 0.0000 \\
SOFA day 3 & $1.3 \pm 1.9$ & 1.2611 & $1.1753-1.3532$ & 0.0000 \\
CrEv day 3 & $0.3 \pm 1.9$ & 2.7951 & $2.0528-3.8056$ & 0.0000 \\
Model III SAPS II & $36.4 \pm 16.3$ & 1.0379 & $1.0253-1.0525$ & 0.0000 \\
SOFA day 5 & $2 \pm 2.3$ & 1.3086 & $1.2047-1.4215$ & 0.0000 \\
CrEv day 5 & $0.3 \pm .4$ & 2.7118 & $1.7977-4.0908$ & 0.0000 \\
\hline
\end{tabular}

Results: Data on 1633 patients were analysed: median age of 69 years; median SAPS II score of 35 and ICU mortality rate of $14 \%$. Including the variables indicated in a logistic regression, three models (Table) could be constructed.

Conclusion: Confirming previous studies, the predictive power of first day SAPS II score decreases over time. The inclusion of intermediate outcomes contributes, significantly, to explain ICU mortality. This study strongly suggests the importance of accurate control of processes of care (and the way of working) in the ICU: showing that the incidence and time spent in out-of-range measurements are clearly associated to the final outcome of ICU-patients.

\section{Reference:}

1. Moreno $\mathrm{R}$ et al:: The use of maximum SOFA score to quantify organ dysfunction/failure in intensive care. Results of a prospective, multi centre study. Intensive Care Med 1999, 25:686-696. 


\section{P237 An alternative, and more sensitive, approach to detecting differences in outcome in sepsis}

\section{RS Wax, WT Linde-Zwirble, M Griffin, MR Pinsky and DC Angus}

Department of Anesthesiology and Critical Care Medicine, University of Pittsburgh School of Medicine; Pittsburgh, PA 15261, USA

When comparing a characteristic (e.g. outcome) between two groups, tests of continuous (as opposed to categorical) data that assume parametric (as opposed to nonparametric) distributions are the most powerful. Currently, we measure outcome differences in sepsis trials in two ways. Typically, we compare mortality rates at a given time-point using categorical, parametric tests (e.g. $\chi^{2}$ or Fisher's Exact test of differences in mortality at day 28) or we compare survival times using categorical, non-parametric tests (e.g. the Log-rank test to compare KaplanMeier curves). But survival after sepsis decreases exponentially [1]. Thus, survival could be described by exponential curves, which can be compared using continuous, parametric tests, such as the Cox's F-test. We therefore used this approach in a cohort of septic patients to determine sample size requirements in comparison to traditional approaches.

\section{Methods:}

Patients: 1102 patients with severe sepsis enrolled in a US multi-center trial.

Sub-groups: We divided patients into those with and without septic shock to select two groups with a difference in survival (10-15\%) typical for many power calculations in sepsis trials.

Statistical procedures: For each sub-group, we plotted the survival to day 28 and fit distributions with exponential curves using the maximum likelihood procedure. Curves were then compared using the Cox's F-test. We also compared differences in outcome using the Fisher's Exact test (for day-28 mortality) and the log-rank test. Statistical significance was assumed at $P<0.05$.

Sampling procedure: After comparing tests on the entire sample, we then drew progressively smaller random
Figure

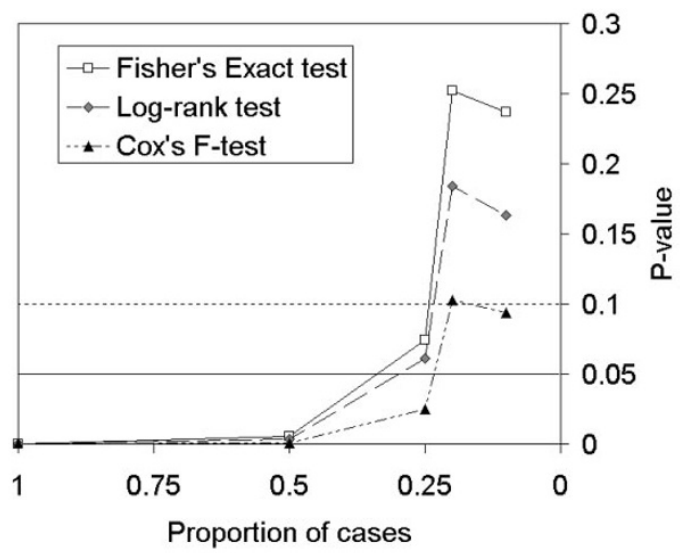

samples of the cohort and repeated the test comparisons to determine the point at which statistical significance was lost for each test.

Results: Patients with shock had a higher mortality than those without shock (see Table). This difference was statistically significant by Fisher's Exact and Log-rank tests until sample size fell below 500. Survival in all sub-groups was modeled by exponential curves with excellent fit $\left(R^{2}\right.$ $>0.98$ ). Comparing these curves by Cox's F-test, statistical significance was maintained with a much smaller sample size (see Fig.).

Conclusion: Taking advantage of the parametric distribution that characterizes survival after sepsis, we can apply a test that finds statistical differences in survival with smaller sample sizes than traditional approaches. These data suggest that the application of exponentially-modeled sur-

Table

\begin{tabular}{|c|c|c|c|c|c|c|c|}
\hline \multirow[b]{2}{*}{ Proportion of cases } & \multicolumn{2}{|c|}{$N$} & \multicolumn{2}{|c|}{ Day 28 mortality (\%) } & \multicolumn{3}{|c|}{$P$-value } \\
\hline & Shock & No shock & Shock & No shock & Fisher's Exact & Log-rank & Cox's F \\
\hline 1 & 621 & 469 & 45.9 & 30.3 & $<0.001$ & $<0.001$ & $<0.001$ \\
\hline 0.5 & 286 & 244 & 44.1 & 32.0 & 0.005 & 0.003 & $<0.001$ \\
\hline 0.25 & 140 & 119 & 42.9 & 31.9 & 0.074 & 0.061 & 0.025 \\
\hline 0.2 & 116 & 91 & 42.2 & 34.1 & 0.252 & 0.184 & 0.103 \\
\hline 0.1 & 59 & 51 & 40.7 & 29.4 & 0.237 & 0.163 & 0.094 \\
\hline
\end{tabular}


vival curve comparisons may be the preferred approach in studies with small sample sizes, such as Phase II trials. Furthermore, this approach may prove to be generally preferable to categorical survival data comparisons, such as day-28 mortality.
Reference:

1. Knaus et al.: JAMA 1993, 270:1233-1241.

P238 Evaluation of the logistic organ dysfunction system for the assessment of organ dysfunction and mortality

PGH Metnitz*, Th Lang ${ }^{\dagger}$, B Kabon*, H Steltzer* and JR Le Gall»

${ }^{*}$ Department of Anesthesiology and General Intensive Care, Univ. Hospital of Vienna, Waehringer Guertel 18-20, A-1090

Vienna, Austria; ${ }^{\dagger}$ Department of Medical Statistics, Univ. of Vienna, Austria; ${ }^{\ddagger}$ Department of Intensive Care Medicine, Saint-Louis

Hospital, Paris, France

Objective: To evaluate the performance of the Logistic Organ Dysfunction (LOD) system for the assessment of morbidity and mortality in multiple organ dysfunction/ failure (MOD/F) in an independent database and to evaluate the use of sequential LOD measurements for the prediction of outcome.

Design: Prospective, multicentric cohort study.

Setting: Thirteen adult medical, surgical, and mixed intensive care units (ICUs) in Austria.

Patients: A total of 2893 consecutive admissions to 13 adult medical/surgical intensive care units (ICUs) in Austria.

Outcome measure: Patient vital status at ICU- and hospital discharge.

Results: Univariate analysis showed that the LOD was able to distinguish between survivors and nonsurvivors ( 2 vs 6 median score, $P<0.05)$. Within organ systems, higher levels of the severity of organ dysfunction were consistently associated with higher mortality. For the prediction of hospital mortality, the original prognostic LOD model did not perform well in our patients as indicated by the goodness-of-fit statistic ( $\mathcal{C}=37.2,9 \mathrm{df}, P<0.0001)$. Using multiple logistic regression, we developed a prognostic model (using the LOD of the first ICU day) with a satisfactory fit in our patients. The integration of further measurements during the ICU stay could not increase the accuracy of the prediction.

Conclusions: The LOD system can be used to quantify the baseline severity of organ dysfunction. Moreover, after customization of the predictive equation, the LOD was able to predict hospital mortality in our patients with high precision. It thus provides a combined measure of morbidity and mortality for critically ill patients with MOD/F.

\section{P239 Predictive accuracy of three scoring systems in a single intensive care unit}

PS Martins and S Blecher

Hospital Santa Marcelina, Rua Costa Gomes, 178 Vila Canaã, CEP 04382-130, São Paulo, Brazil

Objective: To evaluate the predictive accuracy of the severity of three scoring systems - SAPS II, MPM II and LODS - in a single Intensive Care Unit (ICU).

Design: Prospective cohort study.

Setting: General medical and surgical ICU in a tertiary teaching hospital in city of São Paulo, Brazil.

Patients: 318 consecutive, unselected patients over the period from February to June of 1999. Cardiac surgical and burns patients were excluded.

Outcome measure: Patients vital status at ICU discharge.

Interventions: None.
Measurements and statistical analysis: Data required to calculate the patient's predicted mortality by the considered scoring systems were collected. Area under the receiver operating characteristic curve (ROC curve) was calculated to evaluate discrimination of each scoring system. The goodness-of-fit statistic was used to assess calibration of the models and the strength of the association between the predicted and observed outcome.

Main results: The mean age was $55.2 \pm 19$ years. The length of ICU stay was $10.64 \pm 13.09$ days, and the mortality rate was $26.1 \%$. Calibration and discrimination results and the predicted risk of death are shown in the Table.

Conclusions: The results showed good discrimination for all the models. 
Table

\begin{tabular}{lcccc}
\hline & SAPS II & MPM 24 & MPMO & LODS \\
\hline $\begin{array}{l}\text { Predicted risk } \\
\text { of death }\end{array}$ & $21.1 \pm 22$ & $17 \pm 16.7$ & $25.4 \pm 21.4$ & $21.3 \pm 19$ \\
ROC curve & 0.83 & 0.80 & 0.80 & 0.82 \\
Goodness-of-fit & $\begin{array}{l}\mathrm{C}=5.11 ; \\
P=0.276\end{array}$ & $\begin{array}{l}\mathrm{C}=13.67 ; \\
P=0.008\end{array}$ & $\begin{array}{c}\mathrm{C}=3.59 ; \\
P=0.464\end{array}$ & $\begin{array}{l}\mathrm{C}=2.86 ; \\
P=0.581\end{array}$ \\
\hline
\end{tabular}

LODS, MPM 0 and SAPS II showed good calibration, i.e, there were no significant discrepancy between predicted and observed mortality. MPM 24 showed a poor fit.

According to this, MPMO can be used as a predictor of death at patients admission, SAPS II as a predictor of death in the first $24 \mathrm{~h}$ of admission, and LODS as end point in studies of organ dysfunction in our ICU.

Scoring systems need to be evaluated in terms of predictive accuracy within a single institution before applying them to make quality of care assessments.

P240 The predictive value of serial acute physiology score (APS) and simplified acute physiology score II (SAPS II) in post-operative liver transplant patients

Y Arabi, R Goraj, R McIntyre and A Al-Abdulkareem

Departments of Intensive Care and Hepatobiliary Sciences, MC 1425, PO Box 22490, King Fahad National Guard Hospital, Riyadh, 11426, Saudi Arabia

Purpose: To evaluate the predictive value of daily acute physiology score (APS) of the APACHE II system and simplified acute physiology score II (SAPS II) in postoperative liver transplant patients.

Methods: Records of 20 consecutive postoperative liver transplant patients were reviewed. Daily APS and SAPS II scores were calculated for the first three days. Outcome at hospital discharge was documented. Values were compared using paired t-test and Kruskal-Wallis test.

Results: APS and SAPS II were not significantly different between survivors and nonsurvivors on day 1 and 2 but were different on day 3 (see Table). APS and SAPS II decreased from day 1 to 2 in both survivors and nonsur- vivors $(-29 \%$ vs $-31 \%, P=N S$ for APS, $-24 \%$ vs $-21 \%$, $P=$ NS for SAPS II). However, while APS and SAPS II continued to decrease from day 2 to 3 in survivors it increased in nonsurvivors $(-15 \%$ vs $+8 \%, P=0.03$ for APS, $-7 \%$ vs $+5 \%, P=0.26)$.

Conclusions: In the postoperative liver transplant patients, the following are concluded: 1) in contrast to the progressive decrease in APS and SAPS II in survivors, these scores increased in nonsurvivors between day 2 and 3 after an initial drop; 2) day 3 APS and SAPS II scores better differentiated survivors from nonsurvivors than day 1 and 2 scores; 3 ) a mortality prediction system based on day 3 score or on serial scores is likely to be more accurate than the traditional systems using the first $24 \mathrm{~h}$ data.

Table

\begin{tabular}{|c|c|c|c|c|c|c|}
\hline & \multicolumn{3}{|c|}{ APS } & \multicolumn{3}{|c|}{ SAPS II } \\
\hline & Day 1 & Day 2 & Day 3 & Day 1 & Day 2 & Day 3 \\
\hline Survivors ( $n=16$ ) & $16 \pm 4$ & $11 \pm 3^{a}$ & $9 \pm 4^{\mathrm{a}}$ & $47 \pm 11$ & $37 \pm 13^{a}$ & $32 \pm 12^{a}$ \\
\hline Nonsurvivors $(n=4)$ & $21 \pm 7$ & $13 \pm 2$ & $15 \pm 3^{b}$ & $60 \pm 9 b$ & $46 \pm 9$ & $49 \pm 7^{b}$ \\
\hline
\end{tabular}

a: $P<0.05$ compared to previous day, ${ }^{b}: P<0.05$ for survivors compared to nonsurvivors.

P241 TISS 76 and TISS 28: outcome discrimination and correlation with length of ICU/hospital stay in 303 consecutive patients of a medical intensive care unit

\section{U Janssens, C Graf, J Graf and P Hanrath}

Medical Clinic I, RWTH Aachen, Pauwelsstr. 30, 52057 Aachen, Germany

Objectives: TISS 76 (T76) and the simplified version TISS 28 (T28) quantify nursing workload and allow evaluation of severity of illness and, therefore, may be applied for outcome discrimination. The objective of this study was to compare T76 and T28 with regard to outcome discrimination in patients of a medical intensive care unit (ICU) and correlation with ICU length of stay (LOS) and hospital LOS.

Methods: All patients who stayed $>24 \mathrm{~h}$ in ICU were included between 11/97 and 2/98. T76, T28 were collected daily. Discrimination power for survivors (S) and 
nonsurvivors (NS) was assessed by the area under the Receiver Operating Characteristic (AUROC) curve. Relation of both scores on day 1 as well as last measured score before ICU discharge (T76x, T28x) with ICU/hospital LOS and ICU/hospital mortality was assessed with Pearson's correlation and logistic regression.

Results: 303 patients (216 male [71.3\%], 62 \pm 12 years, ICU LOS 3.7 \pm 4.7 days, hospital LOS $15.1 \pm 13.2$ days, SAPS II 26士13) were studied. ICU mortality was $7.6 \%$, hospital mortality was $14.5 \%$. AUROC for T76 day 1 was $0.79 \pm 0.04$ for T28 $0.76 \pm 0.05$. Correlation between
T76/T28 day 1 and ICU/hospital LOS was only weak. T76x and T28x did not correlate with hospital LOS. Risk of death was associated with T76 (odds ratio $1.1,95 \% \mathrm{Cl}$ 1.04 to 1.18) but not with T28 or T76x/T28x.

Conclusion: Outcome discrimination with T76 and T28 on day 1 was reliable. T76 was slightly superior to T28. Correlation with ICU/hospital LOS was only weak. Risk of death was significantly associated with T76 on day 1 . Last measured score before ICU discharge did not correlate with hospital LOS or mortality.

P242 Severity evaluation in acute pancreatitis: the role of SOFA score and general severity scores

R Matos, R Moreno and T Fevereiro

UCIP, Hospital de St António dos Capuchos, Alameda de St. António dos Capuchos, 1150 Lisboa, Portugal

Introduction: Severity stratification in acute pancreatitis has long been a subject of debate. The availability of instruments specific for this pathologic condition lead some intensivists to argue for their use in this condition. However, to the best of our knowledge, no published study competed all these scores with general severity scores and organ failure scores on the same cohort. The objective of this work is to compare six disease-specific scores with two general severity scores (APACHE II and SAPS II) and one organ failure score (Sequential Organ Failure Assessment [SOFA] score) in patients admitted with acute pancreatitis to a mixed medical-surgical ICU.

Material and methods: We analysed all the patients discharged from the UCl from July 11991 to November 30 1999 with a diagnosis of acute pancreatitis. Basic demographic and clinical data were registered, as were outcome at ICU and hospital discharge as well as
APACHE II, SAPS II, SOFA score (at admission, 24 h, 48 $h$ and maximum during ICU stay), admission Ramson score, Ramson score at $48 \mathrm{~h}$, Imrie score, Osborn score, Blamey score, Balthasar score, collected according to the original descriptions. Raw data necessary for the computation of the scores has been registered prospectively, using a proprietary computerised system.

The discriminative power of the scores was evaluated through the use of the area under the Receiver Operating Characteristics (ROC) curve. Two-sample student T-test was used for the comparison of survivors and nonsurvivors. The outcome measure used was vital status at hospital discharge.

Results: During the study period, 49 patients were discharged with a diagnosis of acute pancreatitis. Biliar tract disease $(n=26)$ and alcoholism $(n=8)$ where the most

Table

\begin{tabular}{|c|c|c|c|c|c|}
\hline Score & Mean $\pm S D$ & Survivors mean $\pm S D$ & Non-survivors mean $\pm \mathrm{SD}$ & $P$ & $\begin{array}{l}\text { Area under ROC } \\
\text { curve (SE) }\end{array}$ \\
\hline APACHE II score & $16.8 \pm 9.9$ & $12.1 \pm 6.1$ & $22.7 \pm 10.5$ & $<0.001$ & $0.818 \pm 0.059$ \\
\hline SAPS II score & $37.5 \pm 22.6$ & $25.3 \pm 12.9$ & $52.4 \pm 23.1$ & $<0.001$ & $0.858 \pm 0.056$ \\
\hline SOFA admission & $5.4 \pm 3.8$ & $3.4 \pm 2.5$ & $7.8 \pm 3.8$ & $<0.001$ & $0.822 \pm 0.063$ \\
\hline SOFA $24 \mathrm{~h}$ & $6.2 \pm 4.0$ & $4.3 \pm 2.9$ & $8.6 \pm 3.9$ & $<0.001$ & $0.820 \pm 0.061$ \\
\hline SOFA $48 \mathrm{~h}$ & $5.1 \pm 4.1$ & $3.4 \pm 2.7$ & $7.1 \pm 4.6$ & 0.001 & $0.738 \pm 0.077$ \\
\hline SOFA maximum & $7.4 \pm 4.2$ & $4.9 \pm 2.9$ & $10.4 \pm 3.6$ & $<0.001$ & $0.872 \pm 0.049$ \\
\hline Ramson 0 score & $2.2 \pm 1.2$ & $1.9 \pm 1.1$ & $2.5 \pm 1.4$ & NS & $0.600 \pm 0.083$ \\
\hline Ramson 48 score & $2.3 \pm 1.6$ & $2.0 \pm 1.5$ & $2.7 \pm 1.7$ & NS & $0.617 \pm 0.083$ \\
\hline Imrie score & $4.7 \pm 1.8$ & $4.1 \pm 1.7$ & $5.5 \pm 1.6$ & 0.004 & $0.731 \pm 0.073$ \\
\hline Osborn score & $4.2 \pm 1.6$ & $3.8 \pm 1.7$ & $4.7 \pm 1.4$ & 0.046 & $0.668 \pm 0.078$ \\
\hline Blamey score & $4.4 \pm 1.7$ & $3.8 \pm 1.7$ & $5.2 \pm 1.4$ & 0.003 & $0.745 \pm 0.071$ \\
\hline Balthasar score & $3.4 \pm 1.4$ & $3.5 \pm 1.2$ & $3.4 \pm 1.6$ & NS & $0.497 \pm 0.088$ \\
\hline
\end{tabular}


common aetiologies. In 13 patients no aetiology could be found. Mean age was $53.3 \pm 16.6$ years, lower in survivors than in nonsurvivors $(45.3 \pm 16.0$ vs $63.1 \pm 11.9, P<0.001)$. Median (interquartile range) length of stay in the ICU was 8.09 days (4.0 to 22.1 days), similar in survivors and nonsurvivors $(P=\mathrm{NS})$. Mortality in the ICU was $34.7 \%(17$ patients) with a corresponding hospital mortality of $44.9 \%$ (22 patients). Mean \pm standard deviation in the global population, in survivors and in nonsurvivors are presented in the Table, together with the area under ROC curve \pm standard error for all the scores.
Conclusions: In this cohort of patients with acute pancreatitis, general severity scores and SOFA score presented a better discriminative capability than disease-specific scores, and the results support their use in risk stratification in these patients. If we take into account the lower work needed for the computation of the SOFA score, when compared to APACHE II and SAPS II, our results support the use of SOFA score, both at admission and latter during the ICU stay, for risk stratification in patients with acute pancreatitis.

\section{P243 Therapeutic Intervention Scoring System (TISS) - a method for calculating costs in the intensive care unit (ICU) and intermediate care unit (IMCU)}

I Kaufmann and J Briegel

Department of Anesthesiology, Hospital of the University of Munich, Marchioninistraße 15, D-81377 Munich, Germany

The Therapeutic Intervention Scoring System (TISS) quantifies type and number of intensive care treatments. This system, therefore, indicates the work load of intensive care and may be used for calculating costs in the ICU. In the present study, TISS scoring was prospectively performed daily on 653 patients in a 14-bed multidisciplinary ICU of a 1400-bed university hospital. In addition, we scored 952 patients after major surgical procedures treated in a 4-bed IMCU affiliated to a 24-h recovery room. Besides TISS, we registered age, diagnosis, and length of stay in the unit in each patient. Data were entered from January 11998 to December 31 1998. A retrospective cost analysis was performed. The cost of each TISS point was derived from the division of the costs for clinical support services, consumption and staff for all patients in 1998 and the sum of TISS points of all patients in 1998. To determine the cost of each patient, the TISS point price was multiplied with the sum of daily and total TISS points of the patients.

In the ICU, the total accumulated TISS score was 174950 points. This gave an average cumulative TISS score of 268 in these patients. The median length of stay was 10 days and the median SAPS II score was 23 points. The mortality reached $5.05 \%$. A cost analysis revealed a value of 74.66 DM (38.17 Euro) per TISS point, which can be used to calculate the average costs for groups of patients. The daily cost of ICU care was 3183 DM (1627.44 Euro) per patient in survivors and 4079 DM (2085.56 Euro] in nonsurvivors. For mechanically ventilated patients the daily costs averaged 3404 DM (1740.44 Euro], for patients with spontaneous breathing 2501 DM (1278.74 Euro). Daily cost also depended on age $(<60$ years, 3367 DM [1721.52 Euro]; 60-70 years, 2463 DM [1259.31 Euro]; $>70$ years, 3487 DM [1782.87 Euro]) and length of stay $(<24 \mathrm{~h}, 2733 \mathrm{DM}$ [1397.36 Euro]; 2-4 days, 2613 DM [1336.01 Euro]; $\geq 5$ days, 3658 DM [1870.31 Euro]). Liver transplant recipients consumed 3621 DM (1851.39 Euro) per day, patients after lung transplantation 2971 DM (1519.05 Euro) per day. Patients after major surgical procedures staying less than $24 \mathrm{~h}$ reached daily cost of 2733 DM (1397.36 Euro).

In the IMCU, the total accumulated TISS-score was 39,765 points. This gave an average cumulative TISS score of 42 in these patients. The median length of stay was 1.4 days, the median SAPS II score 18 points and the mortality rate $0 \%$. A cost analysis revealed a value of 78.99 DM (40.39 Euro) per TISS point. Postoperative patients in the intermediate care unit staying $<24 \mathrm{~h}$ consumed 2562 DM (1309.93 Euro) daily.

In conclusion, in this study we could demonstrate an activity based costing methodology which measures the patient-related costs of care in both, ICU and IMCU. The TISS is a valuable tool when evaluating costs in the ICU and in the IMCU. Charging patients to diagnosis related groups allows calculation of costs and resource allocation to different specialties. Comparing the ICU and the IMCU there was a daily cost difference of 171 DM (87.43 Euro) per patient due to a lower workload and lower costs of the nursing staff in the IMCU. These findings support the potential cost saving of managing patients in an IMCU.

\section{P244 Managing cost in ICU - a report of an experience}

CP Souza Paulo, F Leibel and MD Magalhães

ICU Hospital de Clínicas de Niterói, Rua José Higino, 214/1104-CEP: 20520-200, Rio de Janeiro, Brazil

Background: Costs have a huge impact in ICU bills and are the subject of many articles in the medical literature. To reduce costs without decreasing quality is a challenge.
Objectives: To demonstrate a cost management program in a 12-bed ICU of a general hospital with 72 beds. We've chosen three drugs of great impact in the bill and we've 
established a program that rationalized or replaced these drugs and a way to control if there was any negative impact on quality.

Methods: The period of the study was from January 1998 to July 1999. The three drugs chosen were human albumin, omeprazole and midazolan. To decide when and how to use albumin and omeprazole, we employed evidence-based medicine concepts; with midazolan, we changed to diazepam, in equivalent doses, and controlled the "ideal doses" by the Ramsay scale.

Results: We achieved a considerable reduction in global costs, specifically US\$20000 for albumin, US\$3200 for omeprazole and US\$2000 for midazolan, each month. The global economy was US\$277000 a year, without major mortality (compared to predicted by the APACHE II score), increased length of stay (LOS), discomfort or difficulties inside the medical group. The benefits are maintained until now (19 months later).

Conclusion: Control costs without loss of quality is a challenge and a duty. We proved that this is possible without any problem. It's necessary to have clear targets and scientific basis, as evidence-based medicine, in conducting the protocols. Other areas are being included in this approach in our group. 